\title{
Imperfect Repair Strategies for Two-Dimensional Warranty
}

\author{
by \\ Sima Rouhollahi Varnosafaderani
}

\author{
A thesis \\ submitted to the Victoria University of Wellington \\ in partial fulfilment of the \\ requirements for the degree of \\ Master of Science with Honours \\ in Statistics and Operations Research. \\ Victoria University of Wellington \\ 2010
}




\begin{abstract}
When a repairable product under warranty fails, the manufacturer (warrantor) has the choice to either repair or replace the failed product. When repairing a failed product, the degree of repair which affects the working condition of the product can vary, and this is assumed to have an impact on the cost of the repair. The main motivation of this study is to develop a warranty repair strategy that minimizes the costs associated with servicing the warranty.

In this research, the product coverage is represented by a two-dimensional rectangular region with a free-replacement warranty. We propose an imperfect repair strategy that suggests employing imperfect repairs of a predefined degree, in prespecified subregions of the warranty region. The aim is to then minimize the expected warranty servicing cost to the manufacturer by determining the optimal partitioning of the warranty region for the chosen degrees of repair.

Two imperfect repair models are considered, and for both, the expressions for the distribution of the times to imperfect repair and the expected warranty servicing cost per product sold are derived.

We numerically illustrate our findings and compare the expected costs of the proposed imperfect repair strategy with those of previously developed repair-replacement warranty strategies.
\end{abstract}




\section{Contents}

1 Introduction $\quad \mathbf{1}$

1.1 Background and Motivation ............. . . 2

1.2 Problem Statement and Research Objective . . . . . . . . . 2

1.3 Research Scope . . . . . . . . . . . . . . . . 4

1.4 Thesis Outline . . . . . . . . . . . . . . . . . . 4

2 Modeling Warranty Claims 6

2.1 Warranty Policy Classification . . . . . . . . . . . 6

2.2 Rectification Actions and Costs . . . . . . . . . . . . 11

2.2.1 Degree of Repair . . . . . . . . . . . . . . . . . 12

2.2.2 Cost Implications . . . . . . . . . . . . . . . . . . 14

2.3 Warranty Claims in One Dimension . . . . . . . . . . . 15

2.3.1 First Failure of the Original Product . . . . . . . . . . 16

2.3.2 Consecutive Failures of the Product . . . . . . . . 17

2.4 Warranty Claims in Two Dimensions . . . . . . . . . . 26

2.4.1 First Failure of the Original Product . . . . . . . . . 27

2.4.2 Consecutive Failures of the Product . . . . . . . . . 28

2.4.3 The One-Dimensional Approach . . . . . . . . . . 31

3 Review of Literature $\quad 35$

3.1 One-Dimensional Repair-Replacement Strategies . . . . . . . 36

3.2 Two-Dimensional Repair-Replacement Strategies . . . . . . . 38

3.3 Imperfect Repair Strategy . . . . . . . . . . . . . . . . . 44 
4 Age Reduction Model 48

4.1 Model Formulation . . . . . . . . . . . . . . . . . . 48

4.2 Times to Imperfect Repair . . . . . . . . . . . . . . . . . 51

4.3 Warranty Servicing Costs . . . . . . . . . . . . 63

4.3.1 Case A: $r_{1} \leq r_{2} \ldots \ldots \ldots 63$

4.3.2 Case B: $r_{2} \leq r_{1} \ldots \ldots \ldots 77$

5 Intensity Reduction Model 82

5.1 Model Formulation . . . . . . . . . . . . . . . . . 83

5.2 Times to Imperfect Repair . . . . . . . . . . . . . . . . 87

5.3 Warranty Servicing Costs . . . . . . . . . . . . . . . . 92

5.3.1 Case A: $r_{1} \leq \mathbf{r}_{2} \ldots \ldots \ldots 93$

5.3 .2 Case B: $r_{2} \leq r_{1} \ldots \ldots \ldots 103$

6 Numerical Illustration $\quad 107$

6.1 Numerical Example . . . . . . . . . . . . . . . . . . . 109

6.1.1 Repair Costs . . . . . . . . . . . . . . . . 110

6.1 .2 Initial Intensity Function . . . . . . . . . . . . . . . 111

6.1.3 Distribution of the Usage Rate R . . . . . . . . . . . 112

6.1.4 Time and Usage at First Failure . . . . . . . . . . . . 113

6.2 Results for Age Reduction Model . . . . . . . . . . . . . . . 114

6.2.1 Expected Costs for the 3-Subregion Strategy . . . . . 114

6.2.2 Expected Costs for the 4-Subregion Strategy . . . . . 120

6.3 Results for Intensity Reduction Model . . . . . . . . . . . 126

6.3.1 Expected Costs for the 3-Subregion Strategy . . . . 126

6.3.2 Expected Costs for the 4-Subregion Strategy . . . . . 132

6.4 Optimal Warranty Repair Strategy . . . . . . . . . . . . . . 138

7 Conclusion and Future Research 140

$\begin{array}{ll}\text { Bibliography } & 148\end{array}$ 


\section{Chapter 1}

\section{Introduction}

Warranty policies play an important role in the sales of most products and services. A warranty policy is a statement made by the manufacturer that outlines the type and extent of compensation offered to the consumer in the event that the warranted product fails or does not meet the consumer's expectations [6].

In today's competitive market, warranties have become an influential and crucial constituent of manufacturer-consumer transactions. For consumers, when shopping for a product, the warranty often becomes an important factor in making the decision to buy the product, since a warranty is often assumed to be related to the reliability of the warranted product, and also, consumers often seek products with a warranty that assumes lower post-purchase maintenance costs [37]. To a consumer, warranties serve as a guarantee of the quality of a product and an assurance that the manufacturer will be responsible to provide a suitable compensation if the product's performance does not meet the promised standards. However, warranties are not of benefit to the consumers alone. Manufacturers also stand to gain from warranty policies. A warranty that is attractive from the consumer's point of view, and well-planned from the manufacturer's point of view, can increase sales and reduce post-purchase maintenance costs and can therefore, result in larger profits [6]. 


\subsection{Background and Motivation}

Warranties have been extensively investigated by many researchers from various disciplines, and have been of great interest to product manufacturers. For a manufacturer, a warranty may result in costs additional to those associated with the designing and manufacturing of the product and thus, warranties have a considerable effect on the overall returns. Inadequately planned warranty policies often result in manufacturers losing large sums of money. In addition, warranty servicing costs can reach $15 \%$ of net sales and therefore, a slight reduction in these costs can result in substantial savings for manufacturers [34].

Although they have been so widely used and researched, the exact pricing of warranties in most situations is not determinable and should be estimated [14]. Since warranty claims may translate to large amounts of money for manufacturers, devising strategies that minimize these estimated costs are crucial in increasing the profits generated by sales of warranted products.

\subsection{Problem Statement and Research Objective}

Warranties are a significant component of competitive economic success. Effective warranty policy (servicing strategy) planning can warrant profits, but inadequately researched policies and deficient cost analysis may result in losses for the manufacturer. Because of the highly confidential nature of the warranty claims data, it is not always simple to estimate the distribution of warranty servicing costs. Instead, the expected total warranty servicing costs serve as estimates.

This research is aimed at reducing the warranty servicing cost, from the manufacturer's point of view, per product sold by finding an optimal repair strategy that minimizes the expected warranty servicing cost. The warranty considered is a two-dimensional free-replacement warranty and 
the warranted product is assumed to be repairable.

Since an "all minimal" repair model and an "all replacement" model are not realistic and/or feasible options, the suggested repair strategy is to employ imperfect repairs in certain situations in order to lower the warranty servicing cost. Imperfect repairs can be modeled in various ways. A brief literature review of some of the proposed methods is provided in the next chapter. In this research, two different approaches have been employed.

The first approach, the age reduction model, is an adaptation of the virtual age model proposed by Doyen and Gaudoin [16], according to which the imperfect repair reduces the virtual age of the product by an amount proportional to its age immediately prior to the repair, i.e. its age at the time of failure. The class of virtual age models was first proposed by Kijima [29] and it suggests that the product immediately after repair can be described by its virtual age which is smaller than the real age of the product. Here, the efficiency of an imperfect repair is measured by its degree, and for consecutive imperfect repairs, these degrees are not all necessarily the same.

The second approach, the intensity reduction model, is a modification of the model proposed by Chukova et al. [11] which suggests that the failure rate function of the product after an imperfect repair is between the failure rate of the product after a minimal repair and the failure rate of the product right after the previous repair. The reduction in the failure rate function is proportional to the distance between these two failure rates. Like in the age reduction model, the degrees of repairs are not necessarily the same for all consecutive imperfect repairs.

We define the two models by the effect of the imperfect repair on the conditional intensity function of the underlying failure process. For each of the two models, we derive the conditional distributions of successive times to imperfect repair and the expected warranty servicing cost that is to be minimized, and numerically illustrate these results. The imperfect 
repair strategy proposed in this research is a generalization of the repairreplacement strategies proposed by Iskandar et al [21] and Chukova et al. [13], and an extension of the imperfect repair model proposed by Yun and Kang [54].

\subsection{Research Scope}

The development of an effective warranty servicing strategy and careful cost analysis are significant elements in reducing warranty servicing costs and increasing returns from product sales. The imperfect repair strategy suggested in this research aims at reducing the expected warranty servicing cost.

The derived distributions of the times to imperfect repair provide a good framework for warranty claims modeling where the repairs under warranty are imperfect. The expected warranty servicing costs derived in this research can be employed in estimating warranty costs for different degrees of repair. Also, the estimates obtained here can easily be extended to account for non-constant repair costs.

\subsection{Thesis Outline}

The outline of this thesis is as follows: in this chapter we have provided a brief overview of the thesis.

In Chapter 2, we discuss some concepts necessary in understanding and developing warranty policies and also included is an outline of types of rectification actions and their associated costs to the manufacturer. We then discuss the one-dimensional and two-dimensional mathematical modeling approaches used in modeling product failures. We also discuss, in some detail, the concepts of failure rate, intensity function and some important stochastic point processes in the context of warranty claims modeling. In addition, a summary of a few imperfect repair models relevant 
to this research is included.

In Chapter 3, we provide a brief review of the literature pertaining to the modeling and analysis of one-dimensional and two-dimensional warranty policies, while focusing mainly on policies that use the onedimensional approach to modeling warranty claims. The chapter also provides a detailed description of the imperfect repair strategy proposed in this research.

In Chapter 4, we derive the distribution of the times to imperfect repair and the expected total warranty servicing cost for the age reduction model.

In Chapter 5, we derive the distribution of the times to imperfect repair and the expected warranty servicing cost for the intensity reduction model.

In Chapter 6, we present some numerical results to illustrate the effectiveness of the proposed imperfect repair strategy, for both the age reduction and the intensity reduction models. Comparisons with two repairreplacement strategies with minimal repairs and replacements are provided.

In Chapter 7, we present conclusions and discuss some possible future research directions. 


\section{Chapter 2}

\section{Modeling Warranty Claims}

The most fundamental element in the study of warranty analysis is building a mathematical model that adequately describes the warranty claims process. Warranty claims are based on product failures and therefore, modeling the warranty claims process, assuming all claims are valid, is equivalent to modeling the failures that result in the claims. Since failures occur at random along the time continuum, a continuous-time stochastic structure is most appropriate in modeling product failures.

This chapter includes some basic concepts of warranty and the mathematical modeling of the warranty claims process, along with a detailed discussion of the approaches that will be used to accomplish the goals of this research. Before we begin with modeling warranty claims, in the following section, we provide brief notes on the classification of types of warranty, and discuss different rectification actions and their cost implications.

\subsection{Warranty Policy Classification}

All products are produced to perform specific designated functions. Testing each product to ensure that it performs up to standards is neither simple nor feasible. After a process of random testing and analyzing historic 
failure data, certain assumptions are made about the performance and reliability of the product based on which a suitable warranty policy is chosen. There are many types of warranty policies in use today and they can be classified based on several factors. The most common factors based on which warranty policies are classified are the dimension, duration and type of reimbursement which are described in detail in the books on warranty analysis by Blischke and Murthy $[7,6]$. In this section, we provide a summary description of each of the three factors.

\section{Warranty Dimension}

Warranty policies can be classified based on the number of variables that are employed in defining the terms of the policy. Variables typically used to set the warranty terms are time or age of the product, usage of the product, etc. Based on the number of variables, the warranty policy can have one, two or more dimensions. Although a warranty can have multiple dimensions, the one-dimensional and two-dimensional warranties are the ones typically used.

A one-dimensional warranty is based on a single variable usually time from purchase or the age of the product. In this case, the warranty is defined by an interval on the time axis, say $[0, K)$, starting immediately after the purchase of the product or, some times, at the instant the product is first put into use. One-dimensional policies are employed when product failures are attributed to mostly the age of the product.

A two-dimensional warranty, is based on two variables, usually time or age and usage. Here, product failures are often attributed to the age and the amount of usage of the product. In this case, the warranty is defined by a region in two-dimensional space, with time along the $x$-axis and usage along the $y$-axis. Different two-dimensional warranty policies can be defined based on the shape of the warranty region. However, the most commonly used warranty is defined by a rectangular region, usually denoted by $\Omega$, where $\Omega=[0, K) \times[0, L), K$ is the maximum time and $L$ is 
the maximum usage specified in the warranty limits. Here, the warranty expires when either or both limits are exceeded.

Higher-dimensional warranties, based on more than two variables, are more complex and are usually used in few applications, for instance, in plane engines. In this research, we consider products sold with a twodimensional warranty policy specified by a rectangular warranty region. Refer to 'Warranty Cost Analysis' by Blischke and Murthy [6] for detailed descriptions of different warranty region shapes and policies.

\section{Duration of Warranty}

The duration of the warranty can be an important factor in the classification of the warranty policy. The duration of the warranty policy, may change following the repair or replacement of a warranted product, based on which warranty policies can be classified into two categories; renewing and nonrenewing.

A nonrenewing warranty policy has a fixed duration which does not change following a repair or a replacement. The warranty period after repair or replacement is the remaining warranty period of the product.

For a one-dimensional warranty policy, let $[0, K)$ denote the initial warranty period and let $T_{i}$ denote the time of the $i$-th failure, $i \geq 1$. After the $i$-th repair (replacement) within the warranty period, the product is covered by warranty for a period $\left[T_{i}, K\right)$. The $i$-th repair (replacement) of the product is not covered by warranty, if $T_{i-1} \leq K$ and $T_{i}>K$.

For a two-dimensional rectangular warranty policy with warranty region $\Omega=[0, K) \times[0, L)$, let the pair $\left(T_{i}, U_{i}\right)$ denote the age and usage at the $i$-th repair or replacement. The $i$-th repair (replacement) of the product is covered by warranty if $\left(T_{i}, U_{i}\right) \in[0, K) \times[0, L)$. After the $i$-th repair or replacement (in the warranty region), the product is covered by warranty for a time period of length $K-T_{i}$ and for a usage of $L-U_{i}$ units, i.e. over the warranty region $\left[T_{i}, K\right) \times\left[U_{i}, L\right)$. For $i \geq 1$, the $i$-th repair (replacement) of the product is not covered by warranty, if $T_{i}>K$ and/or $U_{i}>L$. 
A renewing warranty policy is one in which the warranty period is extended for a period equal to the original warranty period after each repair or replacement within the warranty period.

For a one-dimensional warranty policy, if the $i$-th product failure is within the warranty period, i.e. $T_{i} \leq K$, the warranty limit is extended to $T_{i}+K$. Therefore, the repaired or replaced product has a warranty period of $K$ units. The warranty expires if the time between failures exceeds $K$ units.

For a two-dimensional rectangular warranty policy, if the $i$-th failure occurs at $\left(T_{i}, U_{i}\right)$ and $T_{i} \leq K$ and $U_{i} \leq L$, after the repair or replacement, the product is covered by warranty over the region

$$
\left[T_{i}, T_{i}+K\right) \times\left[U_{i}, U_{i}+L\right)
$$

The warranty expires when the time between failures exceeds $K$ units and/or the usage between failures exceeds $L$ units.

The duration of the warranty period in case of a renewing warranty policy is a random variable, while the duration of the nonrenewing warranty is constant [6]. Renewing warranties are not commonly used and are less cost-effective than nonrenewing warranty policies. In this research, we consider a nonrenewing rectangular two-dimensional warranty policy.

\section{Type of Reimbursement}

Warranty policies can be broadly classified based on the type of reimbursement into free-replacement warranty (FRW), pro-rata warranty (PRW) and combination warranty which is often a combination of the FRW and the PRW.

Under a free-Replacement warranty policy, the manufacturer agrees to repair or replace the failed product, at no cost to the consumer, provided that the product failures occur within the warranty period (region). This type of warranty is the most commonly used policy applied to consumer 
products such as televisions, electronic components, automobile parts, etc [6].

Under a pro-rata warranty policy, the manufacturer is liable to pay only a portion of the repair or replacement cost of the failed product, if the product failure occurs within the warranty period (region), and the consumer pays the remaining. The amount paid by the manufacturer is usually inversely proportional to the age (or age and usage in the two-dimensional case) of the product. That is, the refund is often a decreasing function of the classifying variables. This type of warranty is used with less expensive non-repairable products such as batteries and automobile tires [6].

A combination warranty policy is a combination of the FRW and the PRW. This type of warranty is often characterized by a period of FRW followed by a period of PRW. Combination warranty policies are some times used to cover multicomponent products where some components are covered by an FRW and others are covered by a PRW [7].

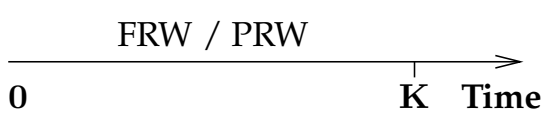

(i)

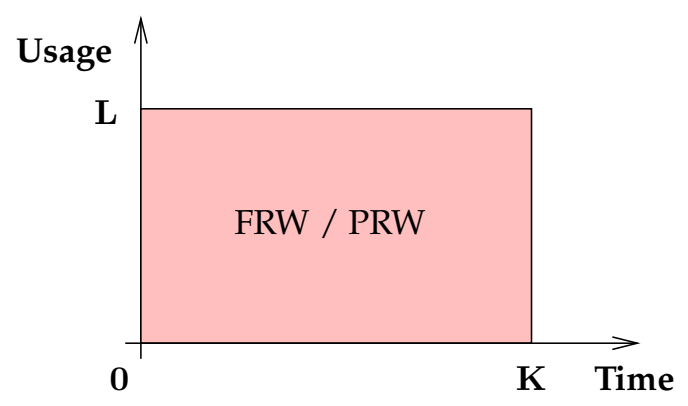

(iii)

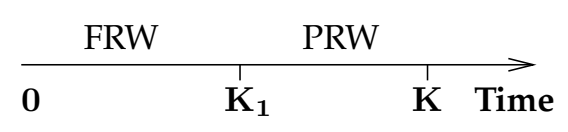

(ii)

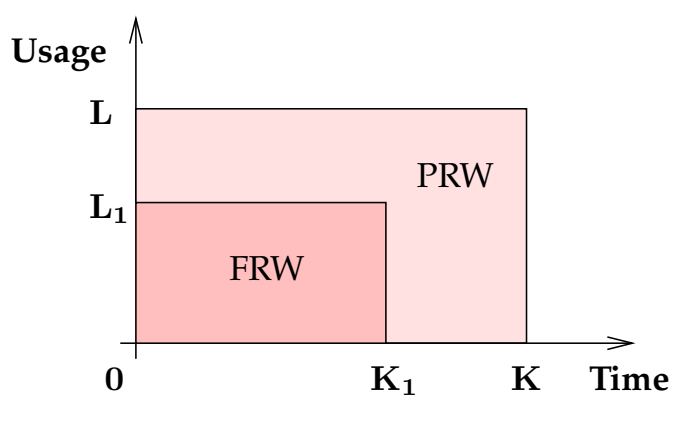

(iv)

Figure 2.1: (i) One-dimensional FRW/PRW, (ii) One-dimensional combination warranty, (iii) Two-dimensional FRW/PRW, (iv) Two-dimensional combination warranty 
Presented in Figure 2.1, are some examples of one-dimensional and two-dimensional warranty policies. In this research, we confine our study to products covered by a nonrenewing two-dimensional FRW policy.

\subsection{Rectification Actions and Costs}

A product is either repairable or nonrepairable. A nonrepairable product is one which is discarded and replaced when it fails to adequately perform and a repairable product is one which can, upon failure, be restored to an acceptable functional state, without necessarily replacing it. Most manufactured products can be repaired on failure, and hence, the study of repairable products is an area of great importance in reliability engineering [45]. In this research, we consider repairable products.

All products degrade with age and/or usage and eventually fail. Product failures can be rectified or controlled through maintenance actions [45]. Maintenance is generally classified into two categories: corrective or preventive. Preventive maintenance refers to the actions performed in order to maintain a product in a specified working condition. Preventive maintenance aims to prevent the occurrence of product failures. Hence, preventive maintenance is done while the product is still in working condition. Corrective maintenance, often termed repair, is any rectification action done to return a failed product to a working condition [46]. In this research, we focus on corrective maintenance or repair.

When a product under warranty fails, the manufacturer can either repair or replace the failed product. If the failed product is nonrepairable, the manufacturer, assuming the warranty claim is valid, can only replace the product. However, if a repairable product fails, the manufacturer can either repair the product or replace it with a new one. The choice between repairing and replacing the failed product depends on many factors such as the cost of the types of rectification action, the working condition of the product prior to failure, the extent of damage, etc $[45,7]$. 
When considering a repairable product, it is frequently assumed that a repair upon failure either returns the product to its original working condition, i.e. renews the product, or has no effect on the working condition of the product, i.e. the repair leaves the product in the condition it was in before failure. These assumptions, in spite making probabilistic modeling less complicated, are not very realistic, since a repair will not always renew a product or leave its working condition unaltered. In more recent studies, the concept of imperfect repair has been the subject of research. Imperfect repair models assume that after repair the working condition of the product is somewhere between the two extreme cases - minimal repair, after which the working condition of the product does not change, and perfect repair or replacement, after which the working condition of the product is the same as a new product. Imperfect repair models are more reasonable and realistic than the minimal repair and replacement models [45].

\subsubsection{Degree of Repair}

The working condition of the repaired product depends on the degree of the repair the product is subjected to. The degree of repair, say $\delta$, is defined as the degree to which a product's working condition can be restored [46]. The degree $\delta$ can be random or preassigned and the range of $\delta$ is usually $[0,1]$. Based on the degree of repair, we use the following three-category classification of repairs from 'Handbook of Reliability Engineering' by Pham [45].

1. Perfect repair or replacement: perfect repair (replacement) restores the operating condition of a failed product to that of a new product. Hence, the lifetime distribution (failure rate) of the product upon repair is the same as the lifetime distribution (failure rate) of a new product. For a degree of repair $\delta \in[0,1]$, a perfect repair (replacement) implies that $\delta=1$. This type of repair can be modeled, under the assumption that the repair time is negligible and can be taken to 
be zero, as an ordinary renewal process. Although modeling replacements of failed products is simple, it is not feasible for the manufacturer to provide replacement products each time the product fails under warranty. Instead, repairing the product to improve its working condition is more feasible.

2. Minimal repair: a minimal repair restores the working condition of the repaired product to the working condition of the product just before failure. The failure rate of the product upon undergoing minimal repair is the same as the failure rate of the product before failure and the degree of repair is $\delta=0$. Minimal repairs can be modeled as a non-homogeneous Poisson process, since the failure rate of the product immediately before failure is the same as the failure rate of the product immediately after repair. From the manufacturer's perspective, minimal repair may be the least costly and hence, most beneficial strategy, but not all failed products can be returned to a working state with minimal repair.

3. Imperfect repair: replacement of a failed product is often not costeffective and minimal repair is not always adequate in returning the failed product to a working condition and hence, we need imperfect repair which restores the product's working condition to an intermediate state, between that of a new product and that of the product prior to failure, i.e. a minimally repaired product. In this research, we assume that the failure rate of the product after imperfect repair is between the failure rate of a new product and the failure rate of a minimally repaired product and hence, for $\delta \in[0,1]$, the degree of repair in case of imperfect repair is $0<\delta<1$. Imperfect repairs can be modeled in various ways and we shall discuss some of these modeling methods later in the chapter.

See Figure 2.2 for the effect of the degree of repair on the failure rate function. The time of the failure is $T_{1}$ and the degree of the imperfect repair 


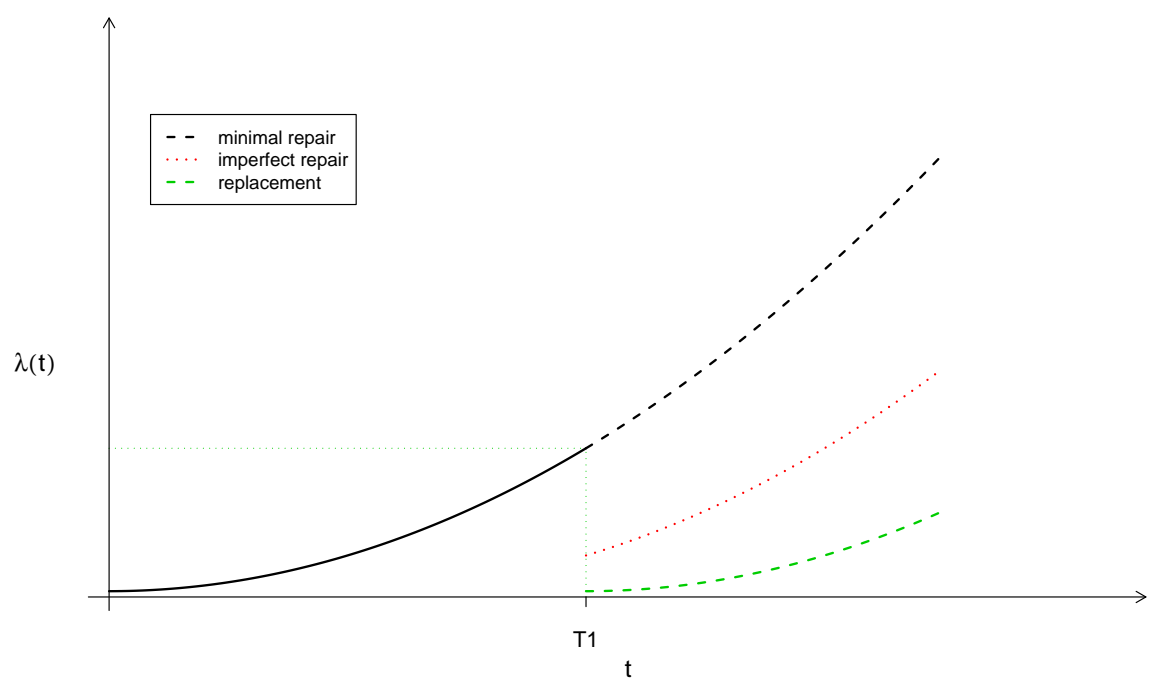

Figure 2.2: Failure rate following repairs of different degrees.

is $\delta=0.5$.

\subsubsection{Cost Implications}

The most important factors in making the choice between repairing or replacing a failed item, are cost and the working state of the product. If the product is nonrepairable or beyond repair, it will have to be replaced by manufacturer at any cost. However, if it is repairable, the manufacturer has the flexibility of choosing the most cost effective option.

When a claim is made under warranty, the manufacturer incurs various costs that are taken into consideration when determining the cost of the repair (replacement). To simplify the modeling process, often, the aggregate of these costs is considered as the cost of the repair. Since the total number of failures and hence, the total number of warranty claims is a random variable, the total warranty servicing cost per product sold which is a function of the number of claims, is also random. Finding a distribution 
function for the total warranty servicing cost is not easy and therefore, it is of importance and convenience to instead derive an expression for the expected total warranty servicing cost [7].

Some of the variables and parameters that affect the warranty servicing cost are the costs associated with different rectification actions and their degrees of repair. The cost of a repair or replacement can be assumed to be proportional to the degree of the repair. Therefore, the degree of repair plays an important role in determining the strategy that minimizes the warranty servicing cost. The degree of repair also has an effect on the number of future failures of the product. In this research, we consider an imperfect repair strategy, where $0<\delta<1$, that aims to minimize the warranty servicing cost.

We now proceed to study the mathematical modeling of the warranty claims process. In the following section, we discuss briefly some one- and two-dimensional stochastic processes, the simplest of which are the Poisson process and the Renewal process, both relevant to modeling the warranty claims process. In modeling warranty claims, it is often assumed that all failures result in immediate and valid claims and repair (or replacement) time is negligible and can be approximated to zero.

\subsection{Warranty Claims in One Dimension}

In the case of one-dimensional warranties, an appropriate model for the warranty claims process is a stochastic counting process that models the product failures along the time continuum. Modeling the lifetime of the original product, i.e. the time to first failure, differs from modeling consecutive failures, since the rectification action performed after the first failure has an effect on the working condition of the product [6]. 


\subsubsection{First Failure of the Original Product}

Let $T_{1}$ denote the lifetime of the original product, i.e., the age of the original product at its first failure. The lifetime $T_{1}$ is a random variable whose distribution is either assumed or derived from the analysis of past failure data. Let $F(t)$ denote the distribution function of the original product. The density function, if it exists, is given by

$$
f(t)=\frac{d}{d t} F(t)
$$

Refer to 'Continuous Univariate Distributions' by Johnson and Kotz [28] for a comprehensive list of distribution functions which are appropriate for modeling product lifetimes. The distribution of the lifetime $T_{1}$ is characterized by the product's failure rate $r(t)$, where $r(t) d t+o(d t)$ is defined as the conditional probability that a product of age $t$ fails in the small interval $[t, t+d t)$, given that the product has not failed prior to $t$. Hence, the failure rate of the product is defined as

$$
\begin{aligned}
r(t) & =\lim _{d t \rightarrow 0} \frac{P\left\{t \leq T_{1}<t+d t \mid T_{1} \geq t\right\}}{d t} \\
& =\lim _{d t \rightarrow 0} \frac{P\left\{t \leq T_{1}<t+d t\right\}}{d t P\left\{T_{1}>t\right\}} \\
& =\frac{1}{\bar{F}(t)} \lim _{d t \rightarrow 0} \frac{F(t+d t)-F(t)}{d t} \\
& =\frac{f(t)}{\bar{F}(t)},
\end{aligned}
$$

where $\bar{F}(t)$ is often called the reliability function of the product. By rearranging the terms in equation (2.1), we get the reliability function of the product in terms of its failure rate:

$$
\begin{aligned}
\frac{d}{d t}\{\ln [\bar{F}(t)]\} & =-r(t) \\
\ln [\bar{F}(t)] & =-\int_{0}^{t} r(s) d s \\
\bar{F}(t) & =\exp \left\{-\int_{0}^{t} r(s) d s\right\} .
\end{aligned}
$$


Given the above relation, the density function $f(t)$ and the distribution function $F(t)$, can be expressed in terms of the failure rate $r(t)$ as follows:

$$
F(t)=1-\exp \left\{-\int_{0}^{t} r(s) d s\right\}
$$

and

$$
f(t)=r(t) \exp \left\{-\int_{0}^{t} r(s) d s\right\} .
$$

The form of the failure rate function $r(t)$ depends on the distribution function and the parameter(s) involved in describing the distribution. Based on $r(t)$, the distribution function $F(t)$ can be classified into one of three categories. The distribution function $F(t)$ has a(n)

- increasing failure rate (IFR), if $r(t)$ increases as $t \geq 0$ increases;

- decreasing failure rate (DFR), if $r(t)$ decreases as $t \geq 0$ increases;

- constant failure rate (CFR), if $r(t)$ remains constant for all values of $t \geq 0[7,4]$.

In this research, we consider a product with an increasing failure rate.

A product upon failure is either repaired or replaced. If the product is replaced upon each failure, the lifetime of each replacement product is the same as the original product. However, if the product is repaired, the future failure times of the product depend on the degree of the repair performed after the previous failure. Consecutive lifetimes or failure times are modeled as stochastic counting processes.

In the following section, we provide a brief introduction to counting processes and their role in modeling consecutive product failures.

\subsubsection{Consecutive Failures of the Product}

Let the number of failures in $[0, t)$ be denoted by $N(t)$, where $N(0)=0$. The process $\{N(t) ; t \geq 0\}$ is a continuous-time non-negative integer valued stochastic process that counts the number of failures that have occurred in the interval $[0, t)$. The point process is such that, if $s<t$, then 
$N(s) \leq N(t)$ and for $s<t, N(t)-N(s)$ denotes the number of failures that have occurred in the interval $[s, t)$. The counting process $\{N(t) ; t \geq 0\}$ is said to have independent increments if for all

$$
t_{0}<t_{1}<t_{2}<\cdots<t_{n}
$$

the random variables,

$$
N\left(t_{1}\right)-N\left(t_{0}\right), N\left(t_{2}\right)-N\left(t_{1}\right), \ldots, N\left(t_{n}\right)-N\left(t_{n-1}\right)
$$

are independent, and the process is said to possess stationary increments if $N(t+s)-N(t)$ has the same distribution for all $t$, i.e. the increments are stationary if the distribution of the change in the process value between any two points, say $t$ and $t+s$, depends only on the distance $s$ between the two points [49].

Let $[0, K)$ denote the warranty period and let $T_{i}$ denote the time of the $i$-th failure, $i=1,2, \ldots$. Then, the total number of failures during the warranty period is $N(K)=\max \left\{i: T_{i}<K\right\}$ or

$$
N(K)=\sum_{i=1}^{\infty} \mathbb{I}\left\{T_{i}<K\right\}
$$

where $\mathbb{I}\left\{T_{i}<K\right\}$ is an indicator function defined as

$$
\mathbb{I}\left\{T_{i}<K\right\}= \begin{cases}1, & T_{i}<K \\ 0, & T_{i} \geq K\end{cases}
$$

Consider a small interval $[t, t+d t)$ and assume that at most one failure can occur in this interval. Therefore, $N(t+d t)-N(t)$ is a binary random variable which can only take the value 0 or 1 . The conditional intensity function $\lambda_{c}(t), t \geq 0$, is defined as the conditional probability that a failure occurs in the interval $[t, t+d t)$, given the history $\mathcal{F}_{t}$ of the process before time $t$, divided by the length $d t$ of this interval. Hence

$$
\lambda_{c}(t)=\lim _{d t \rightarrow 0} \frac{P\left\{N(t+d t)-N(t)=1 \mid \mathcal{F}_{t}\right\}}{d t},
$$


where $N(t+d t)-N(t)$ is the number of failures in the interval $[t, t+d t)$ and $\mathcal{F}_{t}=\{N(s) ; 0 \leq s<t\}$ contains the possible trajectories of the process before time $t$. The rate of occurrence of failures or the intensity function is defined as

$$
\lambda(t)=\lim _{d t \rightarrow 0} \frac{P\{N(t+d t)-N(t)=1\}}{d t}
$$

and hence,

$$
\lambda(t)=E\left[\lambda_{c}(t)\right]
$$

is the mean of the conditional intensity function $\lambda_{c}(t)$ over all the sample trajectories of the failure process $[18,33]$. Since $N(t+d t)-N(t)$ is a binary variable

$$
P\{N(t+d t)-N(t)=1\}=E[N(t+d t)-N(t)] .
$$

From (2.3) and (2.4), it follows that

$$
\lambda(t)=\lim _{d t \rightarrow 0} \frac{E[N(t+d t)]-E[N(t)]}{d t}=\frac{d}{d t} E[N(t)] .
$$

Therefore, the mean function or the cumulative intensity function $\Lambda(t)$ of the process is defined as

$$
\Lambda(t)=E[N(t)]=\int_{0}^{t} \lambda(s) d s .
$$

The failure rate of the product, in terms of the counting process, is defined as

$$
r(t)=\lim _{d t \rightarrow 0} \frac{P\{N(t+d t)-N(t)=1 \mid N(t)=0\}}{d t},
$$

which is a special case of the conditional intensity function in equation (2.2). The first failure of the original item is characterized by the failure rate, while consecutive failures after the first failure are characterized by the conditional intensity function of the underlying counting process. The initial conditional intensity function, i.e. the conditional intensity function prior to the first failure, is the failure rate of the product. That is,

$$
\lambda_{c}(t)=r(t) .
$$


Consider the two extreme cases where all the degrees of repair are either $\delta=0$ (minimal repairs) or $\delta=1$ (replacements). In the following sections, we provide brief discussions on modeling the minimal repair and replacement options.

\section{Modeling Minimal Repairs: Nonhomogeneous Poisson Process}

When a product is minimally repaired upon failure, it's working condition is restored to its condition immediately prior to failure and hence, there is no change in the failure rate function after repair. If the failure rate of the product just after repair is the same as the failure rate immediately before the repair, the failure process can be modeled as a nonhomogeneous Poisson process, with intensity function

$$
\lambda(t)=\lambda_{c}(t)=r(t)
$$

where $r(t)$ is the failure rate of the original product [8]. See Figure 2.3.

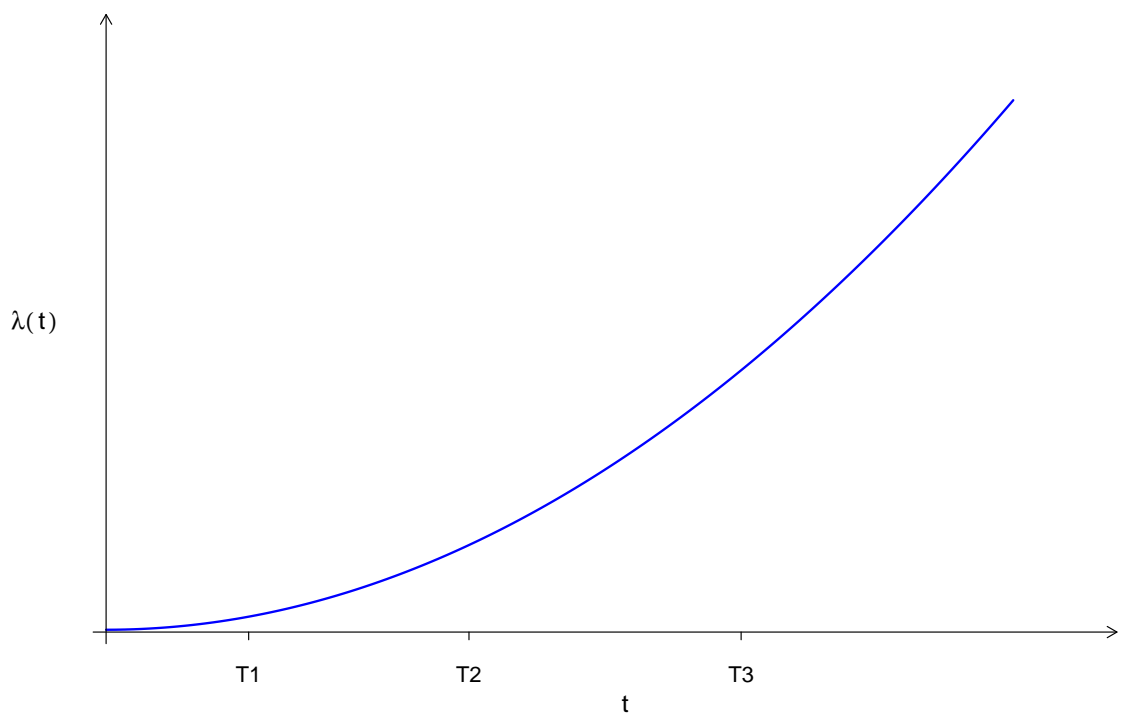

Figure 2.3: Conditional intensity function of a NHPP. 
A counting process $\{N(t) ; t \geq 0\}$ is a nonhomogeneous Poisson process with intensity function $\lambda(t)$ if

1. $N(0)=0$.

2. $\{N(t) ; t \geq 0\}$ has independent increments.

3. $P\{N(t+d t)-N(t)=1\}=\lambda(t) d t+o(d t)$.

4. $P\{N(t+d t)-N(t)>1\}=o(d t)$.

And for $n \geq 0, N(t)$ has a Poisson distribution

$$
P\{N(t)=n\}=\frac{[\Lambda(t)]^{n} e^{-\Lambda(t)}}{n !}
$$

with mean $E[N(t)]=\Lambda(t)$.

If the warranty period is $[0, K)$, then $N(K)$ records the total number of warranty claims in the warranty period, and $\Lambda(K)$ represents the expected number of warranty claims in the warranty period and hence, the expected number of minimal repairs.

\section{Modeling Replacements: Renewal Process}

When a product is replaced upon failure, the failure rate after replacement is the failure rate of the original product. In an all replacement model, consecutive replacements upon failure can be modeled as a renewal process. The initial conditional intensity function of a renewal process is

$$
\lambda_{c}(t)=r(t)
$$

where $r(t)$ is the failure rate of the original product and all replacement products. The conditional intensity function after the first failure is of the form

$$
\lambda_{c}(t)=r\left(t-T_{N(t)}\right),
$$

where $N(t)$ is the number of replacements before time $t$ and hence, $T_{N(t)}$ is the time of the last replacement before time $t[2,17,27]$. See Figure 2.4 . 


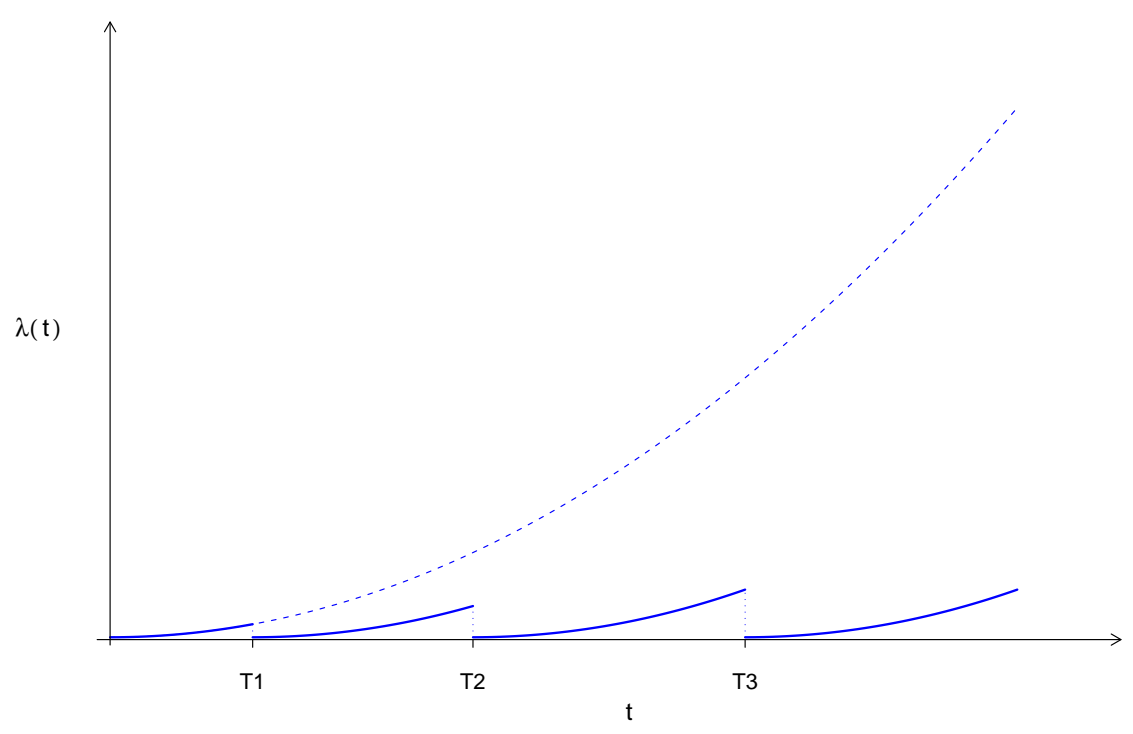

Figure 2.4: Conditional intensity function of a renewal process.

A renewal process $\{N(t) ; t \geq 0\}$ is a counting process that records the number of failures (and hence, renewals) occurring at random in the time interval $[0, t)$. The times between renewals, say $X_{i}, i=1,2, \ldots$, are independent and identically distributed positive random variables with the same distribution function $F($.$) as the original product. The failure times$ $T_{i}, i=1,2, \ldots$ are defined in terms of the lifetimes $X_{i}$ as

$$
T_{i}=\sum_{j=1}^{i} X_{j}
$$

As the distribution of a sum of $n$ independent and identically distributed 
random variables, the distribution of $T_{n}$ is given by

$$
\begin{aligned}
P\left\{T_{n} \leq t\right\} & =P\left\{T_{n-1}+X_{n} \leq t\right\} \\
& =\int_{0}^{\infty} P\left\{T_{n-1}+X_{n} \leq t \mid X_{n}=x\right\} d F(x) \\
& =\int_{0}^{\infty} P\left\{T_{n-1} \leq t-x\right\} d F(x) \\
& =\int_{0}^{\infty} F_{n-1}(t-x) d F(x) \\
& =F^{(n)}(t) \\
& =F(t) * F(t) * \cdots * F(t),
\end{aligned}
$$

where $F^{(n)}(t)$ is the $n$-fold convolution of $F[48,49]$.

In the context of warranty claims modeling, $N(K)$ represents the number of replacements in the warranty period $[0, K)$ and $M(K)=E[N(K)]$, the mean-value function or renewal function, represents the expected number of replacements in the warranty period. For information on estimating the renewal function refer to the works by Jaquette [26], Ross [48] and Xie [51].

\section{Modeling Imperfect Repairs}

Imperfect repair models are more reasonable and realistic than the replacement and minimal repair models. In this section, we briefly discuss some modeling methods, relevant to this research, for imperfect repairs performed on a single-component repairable product.

Kijima [29] proposed two imperfect repair models for a repairable product based on the virtual age process of the product. Suppose that a new product begins operating at time $t=0$, and it is repaired each time it fails. Let the degree of the $i$-th repair be denoted by $\delta_{i}$. Then, $\left\{\delta_{i}\right\}_{i \geq 1}$ is a sequence of independent random variables, where $\delta_{i} \in[0,1]$, for all $i \geq 1$. Two models were proposed based on the effect of the repairs on the virtual age process $\left\{V_{i} ; i \geq 0\right\}$.

The first virtual age model is such that the $i$-th repair cannot undo the damage incurred by the product before the $(i-1)$-th repair, and hence, the additional age $X_{i}$ of the product after the $(i-1)$-th repair is reduced to 
$\delta_{i} X_{i}$. Therefore, the virtual age after the $i$-th repair becomes

$$
V_{i}=V_{i-1}+\delta_{i} X_{i} .
$$

The second virtual age model is such that the $i$-th repair affects the virtual age $V_{i-1}+X_{i}$ accumulated until the $i$-th failure, and the virtual age after the $i$-th repair becomes

$$
V_{i}=\delta_{i}\left(V_{i-1}+X_{i}\right)
$$

That is, each repair removes part of the total accumulated age of the product.

Doyen and Gaudoin [16] introduced two new classes of imperfect repair models. The first is based on a reduction in the conditional intensity function after imperfect repair and the second is based on the reduction in the virtual age of the product after imperfect repair.

In the age reduction models, the effect of the repair is characterized by a reduction in the virtual age of the product and this reduction is proportional to the virtual age of the product at the time of the repair. The real age of the product is $t$ and the virtual age of the product, denoted by $A(t)$, is a positive function of the real age which depends on the past failures and the degrees of the repairs performed following those failures. Therefore,

$$
A(t)=A\left(t ; N(t), T_{1}, T_{2}, \ldots, T_{N(t)}\right),
$$

where $N(t)$ is the number of failures (imperfect repairs) before time $t$. See Figure 2.5.

In the intensity reduction models, the effect of the imperfect repair is characterized by an arithmetic reduction in the conditional intensity function such that at any time $t$ the conditional intensity function is given by

$$
\lambda_{c}(t)=\lambda_{0}(t)-\rho \sum_{j=0}^{N(t)-1}(1-\rho)^{j} \lambda_{0}\left(T_{N(t)-j}\right),
$$

where $\lambda_{0}($.$) is the initial intensity function. The conditional intensity be-$ tween two failures is vertically parallel to the initial intensity. 


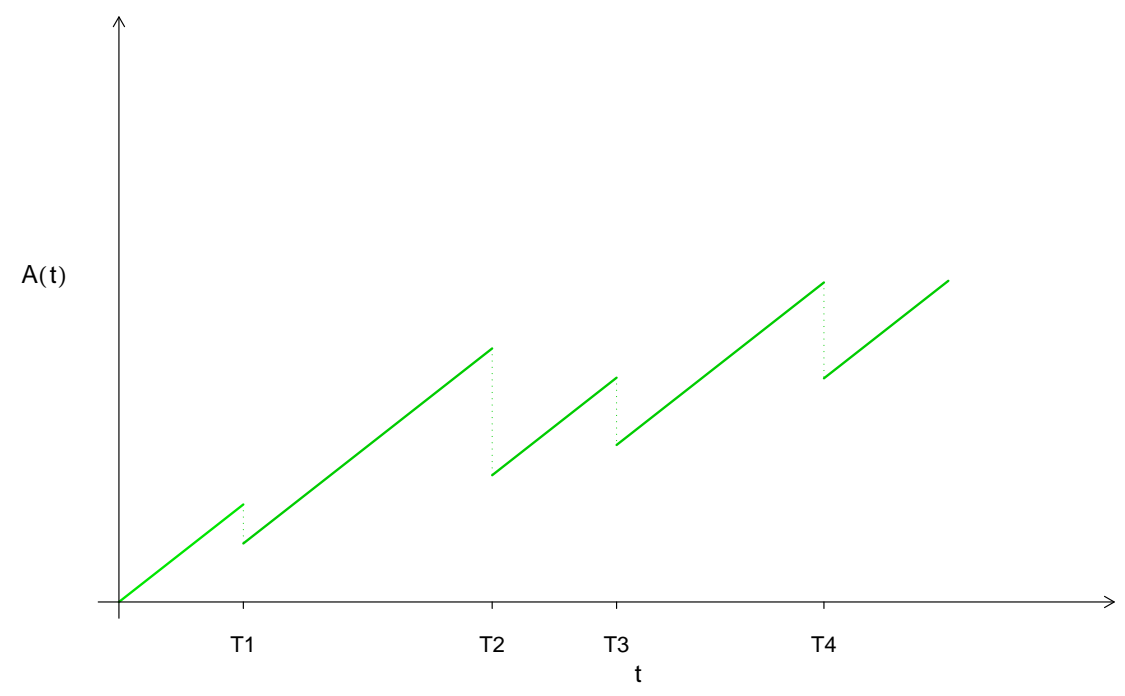

Figure 2.5: Virtual age following imperfect repairs.

Another intensity reduction model is the one introduced by Chukova et al. [11] according to which when a product fails, it undergoes imperfect repair and the conditional intensity after the imperfect repair is between the conditional intensity after a minimal repair and the conditional intensity immediately after the last repair. See Figure 2.6.

Many other imperfect repair models have been suggested. In 'Handbook of Reliability Engineering', Pham [45] provides a good foundation for the study of imperfect repair models. For more on modeling imperfect repairs refer to the works by Nakagawa [40], Brown and Proschan [10], Block et al. [9], Hollander et al. [19], Sheu and Griffith [50], Pham and Wang [46], Jack [23], Langseth and Lindqvist [31] and Mettas and Zhao [35].

In this research, for the first model, we employ an adaptation of the age reduction model proposed by Doyen and Gaudoin [16] which will be discussed in detail in Chapter 4, and for the second model, we use a modification of the model proposed by Chukova et al. [11] which will be 


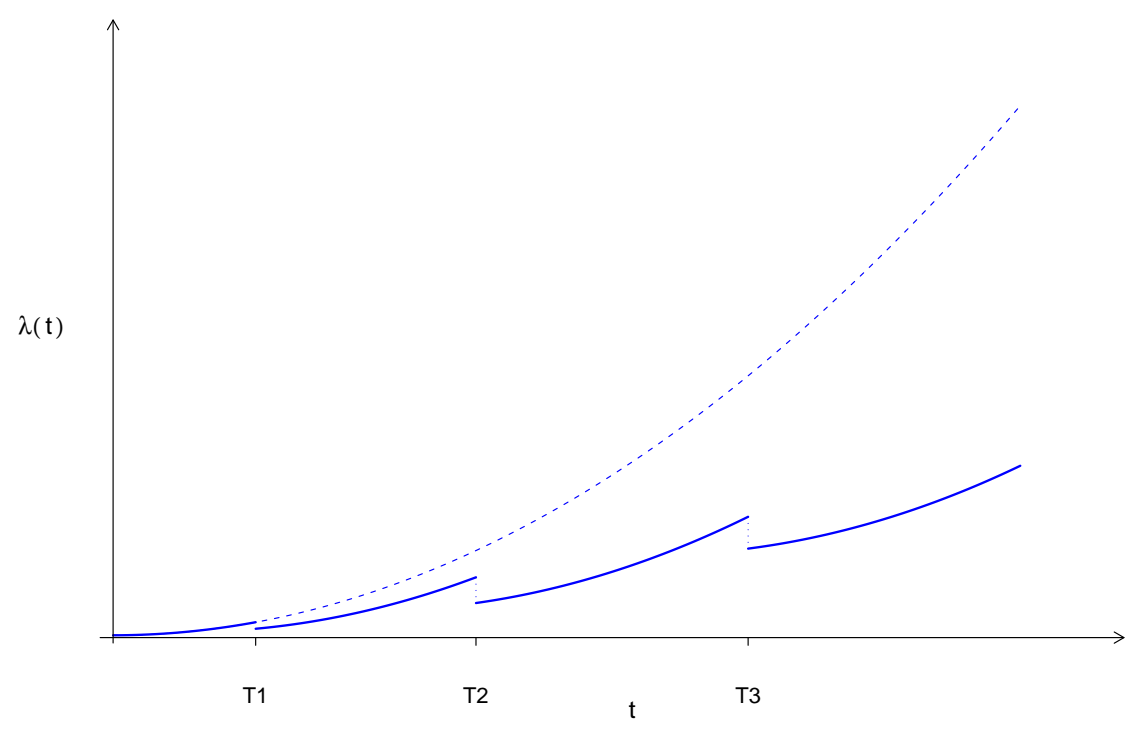

Figure 2.6: Conditional intensity function following imperfect repairs.

discussed in Chapter 5.

\subsection{Warranty Claims in Two Dimensions}

In the case of two-dimensional warranties, the failure process is modeled using a two-dimensional stochastic counting process that counts the product failures in a two-dimensional plane. Two methods have been suggested to model failures in two dimensions: the two-dimensional approach and the one-dimensional approach [38].

Although this research uses the one-dimensional approach which will be discussed in detail later in this section, for completeness, we first briefly discuss the two-dimensional approach. Analogous to the one-dimensional case, we begin with modeling the first failure of the original product and proceed to modeling consecutive failures in two dimensions using the two-dimensional approach. 


\subsubsection{First Failure of the Original Product}

Let $T_{1}$ denote the age of a product at first failure and let $U_{1}$ denote the usage of the product at first failure. In the two-dimensional approach, the pair $\left(T_{1}, U_{1}\right)$ can be modeled as a bivariate random variable with distribution function

$$
F(t, u)=P\left\{T_{1} \leq t, U_{1} \leq u\right\}
$$

and the density function of the distribution (if it exists) is then defined as

$$
f(t, u)=\frac{\partial^{2}}{\partial t \partial u} F(t, u)
$$

The two-dimensional reliability function $\bar{F}(t, u)$, i.e. the probability that the first failure does not occur prior to time $t$ and usage $u$, is defined as

$$
\begin{aligned}
\bar{F}(t, u) & =P\left\{T_{1}>t, U_{1}>u\right\} \\
& =1-\left[P\left\{T_{1} \leq t, U_{1} \leq u\right\}+P\left\{T_{1} \leq t, U_{1}>u\right\}+P\left\{T_{1}>t, U_{1} \leq u\right\}\right] \\
& =1-F_{T_{1}}(t)-F_{U_{1}}(u)+F(t, u)
\end{aligned}
$$

where

$$
F_{T_{1}}(t)=\int_{0}^{t} \int_{0}^{\infty} f(x, u) d u d x
$$

and

$$
F_{U_{1}}(u)=\int_{0}^{u} \int_{0}^{\infty} f(t, y) d t d y
$$

denote the marginal cumulative distribution functions of $T_{1}$ and $U_{1}$ respectively $[1,53]$. In terms of the density function, the reliability function is

$$
\bar{F}(t, u)=\int_{t}^{\infty} \int_{u}^{\infty} f(x, y) d y d x
$$

Analogous to the one-dimensional case, the conditional probability of failure in the rectangle $[t, t+d t) \times\left[u, u+d u\right.$ ) (see Figure 2.7), given that $T_{1}>t$ and $U_{1}>u$ is $r(t, u) d t d u+o(d t d u)$, where $r(t, u)$ is the two-dimensional 
failure rate function defined as

$$
\begin{aligned}
r(t, u) & =\lim _{d t, d u \rightarrow 0} \frac{P\left\{t \leq T_{1} \leq t+d t, u \leq U_{1} \leq u+d u \mid T_{1} \geq t, U_{1} \geq u\right\}}{d t d u} \\
& =\lim _{d t, d u \rightarrow 0} \frac{P\left\{t \leq T_{1} \leq t+d t, u \leq U_{1} \leq u+d u\right\}}{d t d u P\left\{T_{1}>t, U_{1}>u\right\}} \\
& =\frac{1}{\bar{F}(t, u)} \lim _{d t, d u \rightarrow 0} \frac{P\left\{t \leq T_{1} \leq t+d t, u \leq U_{1} \leq u+d u\right\}}{d t d u} \\
& =\frac{f(t, u)}{\bar{F}(t, u)} .
\end{aligned}
$$

The bivariate distribution function $F(t, u)$ can alternatively be derived by

$$
F(t, u)=F_{2}(u \mid t) F_{1}(t),
$$

where $F_{2}(u \mid t)=P\left\{U_{1} \leq u \mid T_{1}=t\right\}$ is the conditional distribution of the usage of the product conditional on the age $T_{1}$ of the product at first failure and $F_{1}(t)=P\left\{T_{1} \leq t\right\}$ is the one-dimensional distribution of $T_{1}$ [6].

\subsubsection{Consecutive Failures of the Product}

Let $\{N(t, u) ; t \geq 0, u \geq 0\}$ denote a two-dimensional stochastic counting process which records the occurrences of failures happening at random in the rectangle $[0, t) \times[0, u)$. If the warranty region is defined by the rectangle $\Omega=[0, K) \times[0, L)$, where $K$ represents maximum time and $L$ represents maximum usage, then $N(K, L)$ denotes the total number of failure (warranty claims) in the warranty region $\Omega$ and is given by

$$
N(K, L)=\sum_{i=1}^{\infty} \mathbb{I}\left\{T_{i}<K, U_{i}<L\right\}
$$

where

$$
\mathbb{I}\left\{T_{i}<K, U_{i}<L\right\}= \begin{cases}1, & T_{i}<K \text { and } U_{i}<L \\ 0, & \text { otherwise }\end{cases}
$$

As in the one-dimensional case, consider the small rectangular region $[t, t+d t) \times[u, u+d u)$ in which at most one failure can occur. For simplicity, 
as in the paper by Baik et al. [2], we use the following notation $N(t, t+d t ; u, u+d u)=N(t+d t, u+d u)-N(t+d t, u)-N(t, u+d u)+N(t, u)$, where $N(t, t+d t ; u, u+d u)$ represents the number of failures in the rectangle $[t, t+d t) \times[u, u+d u)$ (see Figure 2.7).

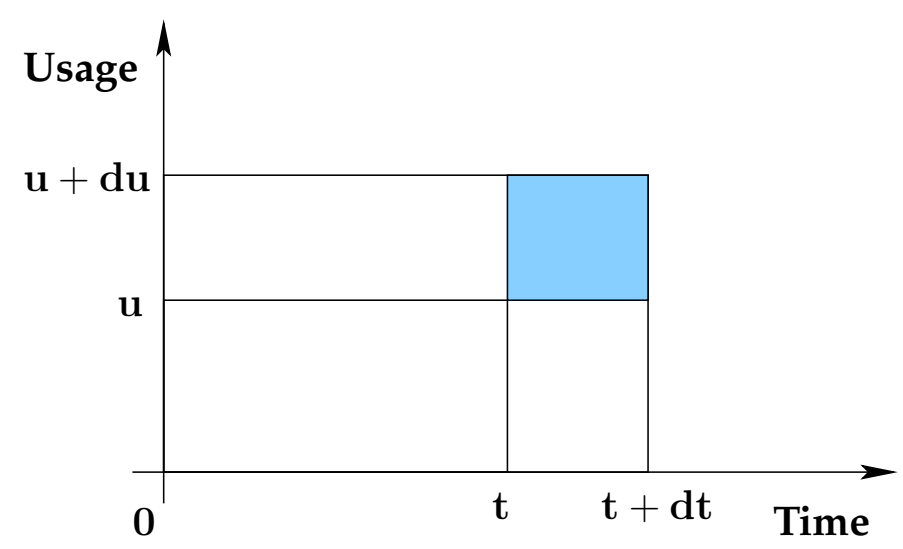

Figure 2.7: The rectangular region $[t, t+d t) \times[u, u+d u)$.

The conditional intensity function of the failure process is given by

$$
\lambda_{c}(t, u)=\lim _{d t, d u \rightarrow 0} \frac{P\left\{N(t, t+d t ; u, u+d u)=1 \mid \mathcal{F}_{t, u}\right\}}{d t d u},
$$

where $\mathcal{F}_{t, u}=\{N(x, y) ; 0 \leq x<t, 0 \leq y<u\}$ is the past of the failure process before time $t$ and usage $u$. The intensity function of the process or the rate of occurrence of failures in $[t, t+d t) \times[u, u+d u)$ is given by

$$
\lambda(t, u)=\lim _{d t, d u \rightarrow 0} \frac{P\{N(t, t+d t ; u, u+d u)=1\}}{d t d u}
$$

and hence, $\lambda(t, u) d t d u$ is viewed as the probability of a failure occurring in the region $[t, t+d t) \times[u, u+d u)$. As in the one-dimensional case, the two-dimensional cumulative intensity function is given by

$$
\Lambda(t, u)=E[N(t, u)]=\int_{0}^{t} \int_{0}^{u} \lambda(x, y) d y d x,
$$


since

$$
\lambda(t, u)=\frac{\partial^{2}}{\partial t \partial u} E[N(t, u)] .
$$

The two-dimensional minimal repairs and replacements models are the nonhomogeneous Poisson process and renewal process in two dimensions respectively. In the following section, we briefly discuss the two models.

\section{Modeling Minimal Repairs: Nonhomogeneous Poisson Process}

Analogous to the one-dimensional modeling of minimal repairs, minimal repairs of a product whose failures depend both on its age and its usage are modeled as a two-dimensional nonhomogeneous Poisson process.

Let $\{N(t, u) ; t \geq 0, u \geq 0\}$ denote a two-dimensional nonhomogeneous Poisson process. Then $N(t, u)$ has a Poisson distribution with intensity function

$$
\lambda(t, u)=\lambda_{c}(t, u)=r(t, u)
$$

That is

$$
P\{N(t, u)=n\}=\frac{[\Lambda(t, u)]^{n} e^{-\Lambda(t, u)}}{n !},
$$

where $\Lambda(t, u)$, defined in equation (2.5), is the expected number of failures in $[0, t) \times[0, u)$.

For the warranty region $\Omega=[0, K) \times[0, L), N(K, L)$ denotes the total number of minimal repairs and $\Lambda(K, L)$ denotes the expected number of minimal repairs in the warranty region. For more, refer to the paper by Baik et al. [2].

\section{Modeling Replacements: Renewal Process}

When all failures are rectified by replacements, the failure process is modeled by a two-dimensional renewal process $\{N(t, u) ; t \geq 0, u \geq 0\}$, with conditional intensity function

$$
\lambda_{c}(t, u)=r\left(t-T_{N(t, u)}, u-U_{N(t, u)}\right),
$$


where $r(.,$.$) is the two-dimensional failure rate function, and T_{N(t, u)}$ and $U_{N(t, u)}$ are the age and usage of the last replacement product in $[0, t) \times[0, u)$ $[2,32,52]$. The number of failures $N(t, u)$ can be expressed in terms of the one-dimensional counting processes corresponding to the age and the usage of the product:

$$
N(t, u)=\min \left\{N_{1}(t), N_{2}(u)\right\}
$$

where $N_{1}(t)$ is the number of failures in the time interval $[0, t)$, and $N_{2}(u)$ is the number of failures in the usage interval $[0, u)[6,18]$. Let the nonnegative bivariate variable $\left(X_{i}, Y_{i}\right)$ denote the age and usage of the $i$-th product, $i \geq 1$. The sequence $\left\{\left(X_{i}, Y_{i}\right) ; i \geq 1\right\}$ is a sequence of independent and identically distributed bivariate random variables, all with the same joint distribution function

$$
F(x, y)=P\left\{X_{i} \leq x, Y_{i} \leq y\right\} .
$$

The failure time-usage pairs $\left(T_{i}, U_{i}\right), i \geq 1$, are the sum of the interfailure time-usage pairs $\left(X_{i}, Y_{i}\right)$ :

$$
\left(T_{i}, U_{i}\right)=\left(\sum_{j=1}^{i} X_{j}, \sum_{j=1}^{i} Y_{j}\right) .
$$

For the rectangular warranty region $\Omega, N(K, L)$ represents the total number of replacements in the warranty region and $M(K, L)=E[N(K, L)]$ represents the expected number of replacements in $\Omega$.

A two-dimensional failure process can be reduced to a one-dimensional process by assuming a relationship between the two variables of the process. In the following section, we discuss the one-dimensional approach to modeling warranty claims in two dimensions.

\subsubsection{The One-Dimensional Approach}

In the context of warranty analysis, the two variables characterizing the two-dimensional warranty region are the age and usage of the warranted 
product. Let $A(t)$ and $U(t)$ denote the age and usage of the product at time $t$, and let $U_{\text {total }}(t)$ denote the total usage of the product at time $t$ [14]. Let the warranty begin at the moment the product is first put into use, $t=0$, which is assumed to be the time of the sale of the product. At this time, the age and usage of the product are both taken to be zero. If no replacements have occurred in $[0, t)$, then $A(t)$ and $U(t)$ are the age and usage of the original item, and $A(t)=t$ and $U(t)=U_{\text {total }}(t)$. If the product has been replaced at least once in the interval $[0, t)$, then $A(t)$ and $U(t)$ are the age and usage of the replacement product in use at time $t$, and $A(t)<t$ and $U(t)<U_{\text {total }}(t)$.

In the one-dimensional approach, we assume that the usage of the product is a linear function of the age of the product, such that

$$
U(t)=R A(t),
$$

where the coefficient $R$ is the usage rate which varies among users and is a non-negative random variable with some distribution $G$. The usage rate $R$, for a given user, is assumed to be constant over the warranty region $[6,14]$. The distribution function and density function (if it exists) of $R$ are given by

$$
G(r)=P\{R \leq r\}
$$

and

$$
g(r)=\frac{d}{d r} G(r)
$$

respectively. The appropriate form for $G(r)$ is chosen based on the consumer usage rate across the population. Blischke and Murthy [6] suggest the Beta, Gamma and uniform distributions for the usage rate $R$.

Let $N(K, L)$, where $K$ and $L$ are the time and usage limits defined in the warranty policy, denote the number of failures in the warranty region $\Omega=[0, K) \times[0, L)$, and let $N(K, L \mid r)$ denote the number of failures in $\Omega$ conditional on $R=r$. Then, the distribution of $N(K, L)$ is given by

$$
P\{N(K, L)=n\}=\int_{0}^{\infty} P\{N(K, L \mid r)=n\} d G(r)
$$




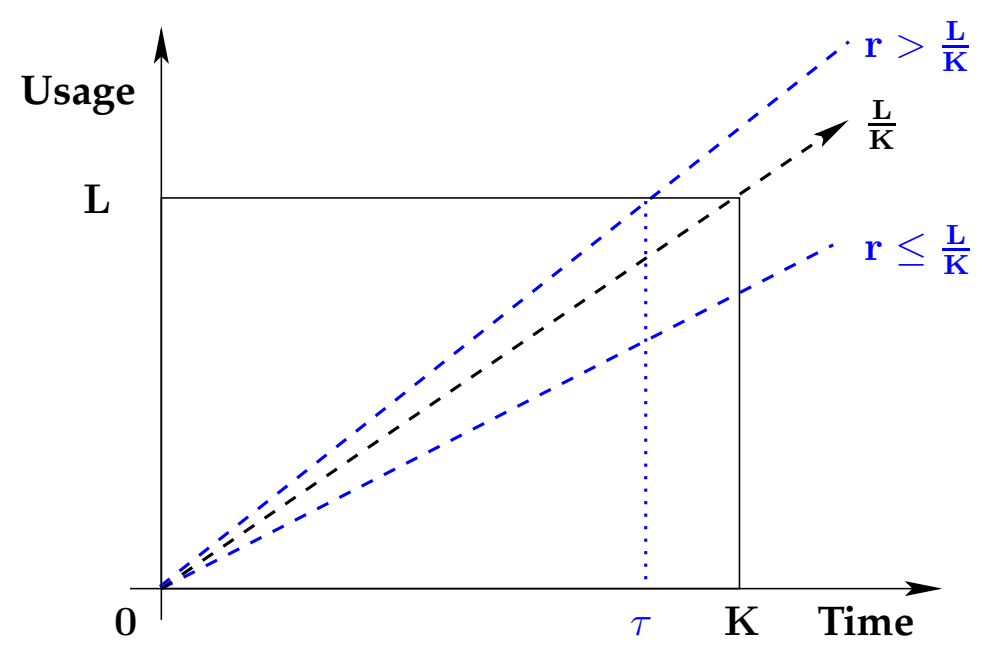

Figure 2.8: A rectangular two-dimensional warranty region.

and since age and usage are related, $N(K, L \mid r)$ can be expressed in terms of a one-dimensional counting process as follows

$$
N(K, L \mid r)= \begin{cases}\tilde{N}(K \mid r), & \text { if } r \leq \frac{L}{K} \\ \tilde{N}(\tau \mid r), & \text { if } r>\frac{L}{K}\end{cases}
$$

where $\{\tilde{N}(t \mid r) ; t \geq 0\}$ denotes the one-dimensional failure process conditional on $R=r$ and

$$
\tau=\frac{L}{r}
$$

See Figure 2.8. The expected number of failures in the warranty region $\Omega$ is given by

$$
E[N(K, L)]=\int_{0}^{\infty} E[N(K, L \mid r)] d G(r)
$$

where $E[N(K, L \mid r)]$ is the expected number of failures in the warranty region conditional on $R=r$ and it can be expressed in terms of the onedimensional process $\{\tilde{N}(t \mid r) ; t \geq 0\}$ as follows:

$$
E[N(K, L \mid r)]= \begin{cases}E[\tilde{N}(K \mid r)], & \text { if } r \leq \frac{L}{K} \\ E[\tilde{N}(\tau \mid r)], & \text { if } r>\frac{L}{K}\end{cases}
$$


When all repairs are minimal, $\tilde{N}(t \mid r)$ is a nonhomogeneous Poisson process with intensity function $\tilde{\lambda}(t \mid r)$ equal to the failure rate function of the original product. In case of replacements, $\tilde{N}(t \mid r)$ is a renewal process with interfailure times having the distribution function

$$
F(t \mid r)=1-\exp \left\{-\int_{0}^{t} \rho(s \mid r) d s\right\}
$$

where $\rho($.$) is the failure rate function of the original product. For further$ details refer to the warranty analysis books by Blischke and Murthy [6, 7].

Thus, one can model the two-dimensional failure process with a onedimensional point process. In this research, we use the one-dimensional approach to model failures in the two-dimensional warranty region $\Omega$. 


\section{Chapter 3}

\section{Review of Literature}

Most studies on warranty policy development and analysis assume that the failures of the warranted product depend only on the age of the product. However, for some products, the failures depend on more than one variable: for example, in automobiles the degradation depends on both the age and usage (mileage) of the vehicle. Studies on the modeling and analysis of two-dimensional warranty policies are a fairly recent addition to the warranty literature.

In this chapter, we provide a brief review of the literature on onedimensional and two-dimensional warranty policies. We focus mostly on cost-minimizing (for the manufacturer) repair-replacement strategies for two-dimensional free-replacement warranty policies where product failures are modeled using the one-dimensional point process approach as it is more relevant to this research.

In the previous chapter, we reviewed some concepts relevant to warranty policies and their classification. A taxonomy of different warranty policies is given in 'Warranty Cost Analysis' by Blischke and Murthy [6]. As a review of types of warranty policies, Figure 3.1 displays part of this taxonomy. 


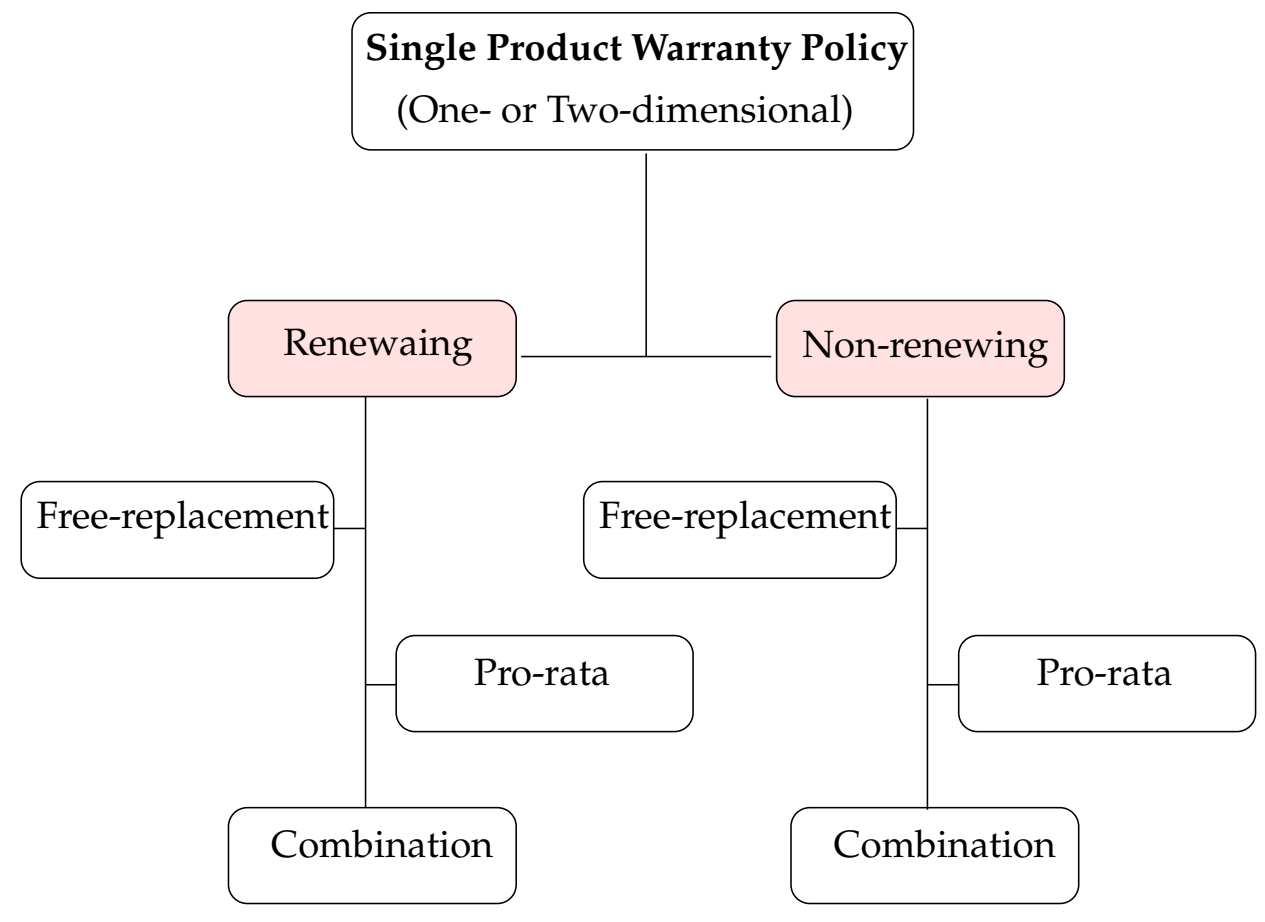

Figure 3.1: Taxonomy for warranty policies [6]

\subsection{One-Dimensional Repair-Replacement Strate- gies}

A one-dimensional warranty policy is characterized by an interval on the time line representing the real age (time) of the product and this interval, starting at the time of the sale of the product, is called the warranty period. Most repair-replacement strategies for the one-dimensional case have been based on minimal repairs and/or replacements. Few strategies have been suggested for imperfect repairs of degree $0<\delta<1$. In this section, we review some one-dimensional repair-replacement strategies.

Nguyen and Murthy [42] proposed a repair-replacement warranty policy for repairable products with an increasing failure rate, where the warranty period $[0, K)$ is divided into two subintervals and all products that 
fail in the first subinterval are replaced by new products and all products that fail in the second subinterval are replaced by repaired products. When a product fails for the first time, if its age is less than some predefined threshold, it is minimally repaired and added to the collection of repaired products to be used as a replacement product. The replacements are done at no cost to the consumer (also see the study by Biedenweg [5]).

Nguyen and Murthy [43] suggested a model to make optimal decisions regarding replace-repair strategies for a combination failure-free warranty policy with fixed and renewed periods $K$ and $W$. The period $(0, K]$ is divided into two intervals and if the product fails in the first interval it is replaced by a new one and if it fails in the second interval it is repaired both free of charge to the consumer. If the product fails in the interval ( $K, K+W$ ], it is either repaired or replaced free of charge to the consumer and the replacement product is covered by a new warranty with a renewed period $W$. When $W=0$ the warranty policy corresponds to a failure-free warranty with a fixed period $K$, and when $K=0$ the warranty policy corresponds to a failure-free warranty with a renewed period $W$.

Nguyen [41] proposed two repair-replacement strategies, in each the warranty period $[0, K)$ is divided into two subintervals $\left[0, K_{1}\right)$ and $\left[K_{1}, K\right)$. The first strategy is such that if a product fails in the first subinterval, it is replaced by a new one and if a product fails in the second subinterval, it is minimally repaired. Under the second strategy, if a product fails in the first subinterval, it is minimally repaired and if a product fails in the second subinterval, it is replaced by a new one. The decision variable $K_{1}$, in each of the two strategies, is selected by minimizing the corresponding warranty servicing cost.

Jack and Van der Duyn Schouten [24] discussed the form of a repairreplacement strategy that minimizes the expected cost of servicing the warranty over the warranty period, where repairs are minimal. They showed that the optimal repair-replacement strategy is determined by comparing the age of the product with a control limit function which is depen- 
dent on time. If the product fails within the warranty period, it is minimally repaired if and only if its age is less than this function. Therefore, the number of replacements is determined by the shape of the control limit function.

Jack and Murthy [25] proposed a new repair-replacement strategy for products sold with a non-renewing free replacement warranty policy by splitting the warranty period $[0, K)$ into three subintervals $\left[0, K_{1}\right),\left[K_{1}, K_{2}\right)$ and $\left[K_{2}, K\right)$, where $0 \leq K_{1} \leq K_{2} \leq K$. All product failures in the first and last subintervals are rectified by minimal repair. At the first product failure in the middle subinterval $\left[K_{1}, K_{2}\right)$, the failed product is replaced by a new one and all subsequent product failures are rectified by minimal repairs.

Yun et al. [55] consider a variation of the strategy proposed by Jack and Murthy [25]. The warranty period $[0, K)$ is still split into three subintervals $\left[0, K_{1}\right),\left[K_{1}, K_{2}\right)$ and $\left[K_{2}, K\right)$, where $0 \leq K_{1} \leq K_{2} \leq K$. All product failures in the first and last subintervals are rectified by minimal repair. At the first product failure in the middle subinterval $\left[K_{1}, K_{2}\right)$, the failed product is rectified by imperfect repair instead of replacement. All subsequent product failures in the middle subinterval are rectified by minimal repair.

For some earlier repair-replacement corrective and preventive maintenance warranty strategies refer to the works by Barlow and Hunter [3], Park [44], Phelps [47], and Mettas [35].

\subsection{Two-Dimensional Repair-Replacement Strate- gies}

A two-dimensional warranty is characterized by a region $\Omega \subset \mathbf{R}_{+}^{2}$ in a two-dimensional plane with the horizontal axis usually representing the age of a product and the vertical axis usually representing the usage of the product. The limits of the warranty coverage are known and fixed. The failures of the product are represented by points in the two-dimensional 
age-usage plane. We confine our research to the case where the warranty region is the rectangular area $\Omega=[0, K) \times[0, L)$, where the warranty expires either at time $K$ or when the total usage of the product exceeds $L$.

Two approaches have been proposed for modeling failures in two dimensions: the one-dimensional and the two-dimensional approaches. In the two-dimensional approach, the product age and the product usage are a bivariate random variable modeled with a bivariate distribution. In the one-dimensional approach, the usage of the product is considered to be an increasing linear function of the age of the product.

Baik et al. [2] extended the concept of minimal repair form one dimension to two dimensions. They provide comparisons between the one- and two-dimensional failure models for an all minimal repair strategy (nonhomogeneous Poisson process) and an all replacement strategy (renewal process). For more on the two-dimensional approach to failure modeling refer to the works by Yang and Nachlas [53], Murthy et al. [39], Kim and Rao [30].

Repair-replacement strategies corresponding to warranty regions with multiple subregions are categorized as either restricted or unrestricted. The definitions are as follows.

Definition 1 Consider a warranty region $\Omega$ divided into $n$ disjoint subregions, $\Omega_{1}, \Omega_{2}, \ldots, \Omega_{n}$ such that

$$
\bigcup_{i=1}^{n} \Omega_{i}=\Omega, \quad \text { and } \quad \bigcap_{i=1}^{n} \Omega_{i}=\emptyset
$$

where

$$
\Omega_{i}=\left\{\left[0, K_{i}\right) \times\left[0, L_{i}\right)\right\} \backslash\left\{\left[0, K_{i-1}\right) \times\left[0, L_{i-1}\right)\right\} .
$$

for $1 \leq i \leq n$, and $K_{0}=L_{0}=0, K_{n}=K$ and $L_{n}=L$. The corresponding repair-replacement strategy, say $\mathcal{S}_{n}$, is restricted if

$$
\frac{L_{1}}{K_{1}}=\frac{L_{2}}{K_{2}}=\ldots=\frac{L_{n-1}}{K_{n-1}}=r_{1}
$$


and

$$
\frac{L_{n}}{K_{n}}=\frac{L}{K}=r_{2}
$$

where $r_{1}>0$ and $r_{2}>0$. In other words, the warranty strategy is restricted if, for $i=1,2, \ldots, n-1$, the rectangles

$$
\bigcup_{j=1}^{i} \Omega_{j}=\left\{\left[0, K_{i}\right) \times\left[0, L_{i}\right)\right\}
$$

are all of similar shape.

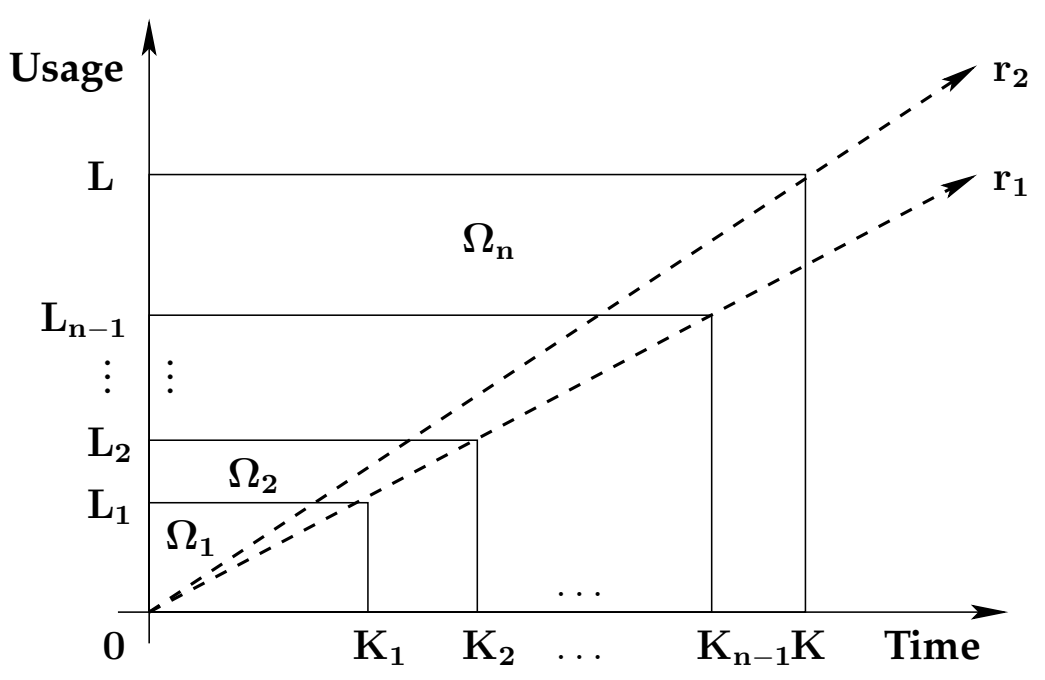

Figure 3.2: Warranty region for the restricted case where $r_{1} \leq r_{2}$.

Definition 2 The n-subregion strategy $\mathcal{S}_{n}$ is unrestricted if all the rectangles

$$
\bigcup_{j=1}^{i} \Omega_{j}=\left\{\left[0, K_{i}\right) \times\left[0, L_{i}\right)\right\},
$$

where $i=1,2, \ldots, n-1$, are not similar in shape.

See Figure 3.2 and Figure 3.3 for the restricted strategy when $r_{1} \leq r_{2}$ and $r_{2} \leq r_{1}$, respectively, and see Figure 3.4 for the unrestricted strategy when $r_{1} \leq r_{2} \leq \ldots \leq r_{n}$. 


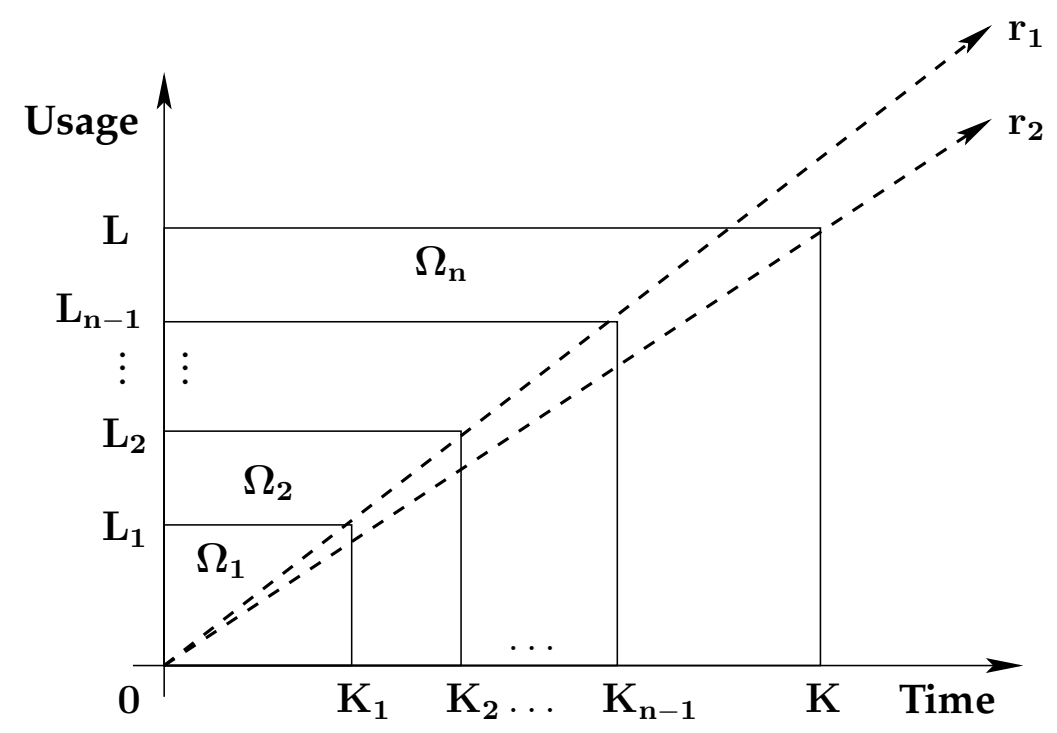

Figure 3.3: Warranty region for the restricted case where $r_{2} \leq r_{1}$.

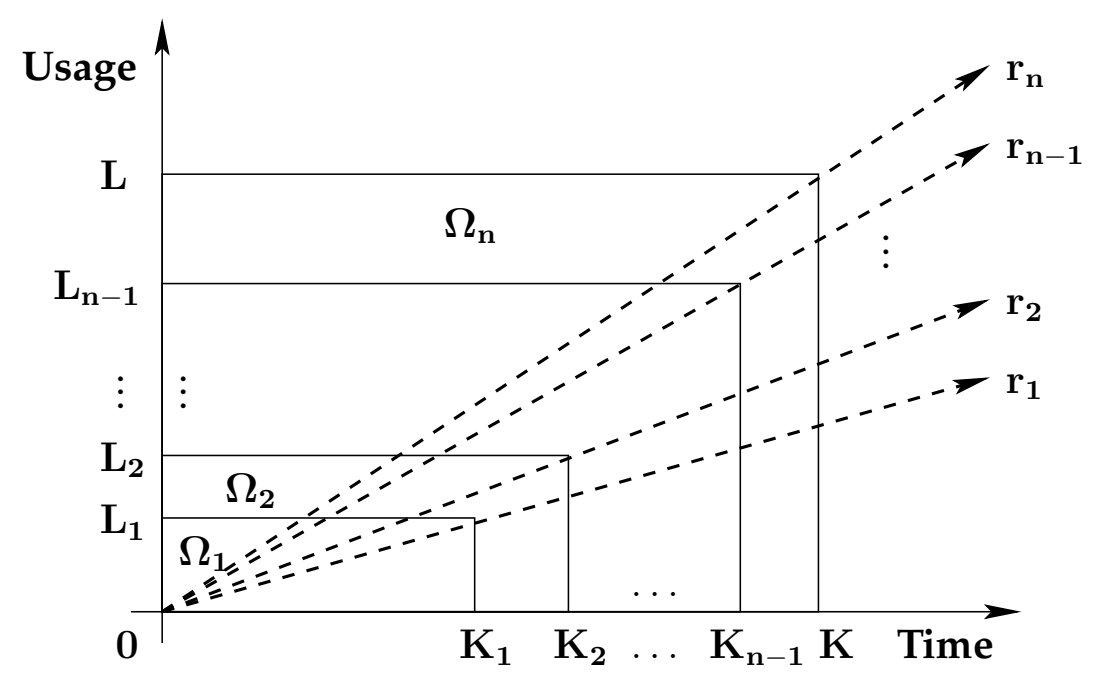

Figure 3.4: Warranty region for the unrestricted case where $r_{1} \leq r_{2} \leq \cdots \leq r_{n}$.

What follows is a review of some previously developed two-dimensional repair-replacement strategies, where the failures are modeled using the one-dimensional approach. 
Moskowitz and Chun [36] proposed a Poisson regression model for two-dimensional warranty policies. They assume that the number of failures of the product is distributed as Poisson and the conditional intensity function is a linear function of the age and usage of the product.

Iskandar and Murthy [20] proposed two two-dimensional free-replacement warranty policies for a repairable product, where in both policies the rectangular warranty region $\Omega=[0, K) \times[0, L)$ is divided into two disjoint subregions $\Omega_{1}$ and $\Omega_{2}$, such that $\Omega_{1} \cup \Omega_{2}=\Omega$ and $\Omega_{1}=\left[0, K_{1}\right) \times\left[0, L_{1}\right)$ is also a rectangle. In the first strategy, if a product fails in $\Omega_{1}$, it is replaced by a new one and if a product fails in $\Omega_{2}$, it is minimally repaired. In the second strategy, if a product fails in $\Omega_{1}$, it is minimally repaired and if a product fails in $\Omega_{2}$, it is replaced by a new one. These are extensions of the one-dimensional repair-replacement strategies proposed by Nguyen and Murthy $[43,42]$ and Nguyen [41]. The decision variables $K_{1}$ and $L_{1}$, in each policy, are determined by minimizing the associated warranty servicing cost. Product failures are modeled, conditional on the usage rate $R=r$, as a Poisson process. This is a more general case of the formulation studied by Moskowitz and Chun [36].

Iskandar et al. [21] extended the one-dimensional warranty servicing strategy proposed by Jack and Murthy [25] to a two-dimensional warranty policy for repairable products sold under free-replacement warranty. They consider a rectangular warranty region with three disjoint subregions $\Omega_{1}$, $\Omega_{2}$ and $\Omega_{3}$, such that $\Omega_{1} \cup \Omega_{2} \cup \Omega_{3}=\Omega$. The rectangles $\Omega_{1}$ and $\Omega_{1} \cup \Omega_{2}$ are assumed to be similar in shape. Hence, the strategy is restricted. If the product fails in $\Omega_{1}$ or $\Omega_{3}$, it is minimally repaired. The first product failure in $\Omega_{2}$ will be rectified by replacing the failed product by a new one, and all subsequent product failures in $\Omega_{2}$ will be rectified by minimally repairing the failed product. The optimal $K_{1}, K_{2}, L_{1}$ and $L_{2}$ are obtained by minimizing the expected warranty servicing cost.

Chukova and Johnston [14] propose an extension of the strategy proposed by Iskandar et al. [21] where the rectangles $\Omega_{1}$ and $\Omega_{1} \cup \Omega_{2}$ are not 
necessarily similar in shape and hence, the strategy is both unrestricted and restricted, i.e. they consider both the case where

$$
\frac{L_{1}}{K_{1}} \neq \frac{L_{2}}{K_{2}},
$$

as well as the case where

$$
\frac{L_{1}}{K_{1}}=\frac{L_{2}}{K_{2}}
$$

The repair-replacement strategy is the same: product failures occurring in $\Omega_{1}$ or $\Omega_{3}$ are rectified by minimal repairs. The first product failure in $\Omega_{2}$ is rectified by replacement of the failed product, and all subsequent failures in $\Omega_{2}$ are rectified by minimal repair. Let

$$
\frac{L_{1}}{K_{1}}=r_{1}, \quad \frac{L_{2}}{K_{2}}=r_{2}, \quad \text { and } \quad \frac{L}{K}=r_{3} .
$$

They derive the expected warranty cost per unit product sold for six cases based on the usage rates $r_{1}, r_{2}$, and $r_{3}$ :
(1) $r_{1} \leq r_{2} \leq r_{3}$
(2) $r_{1} \leq r_{3} \leq r_{2}$
(3) $r_{2} \leq r_{1} \leq r_{3}$
(4) $r_{2} \leq r_{3} \leq r_{1}$
(5) $r_{3} \leq r_{1} \leq r_{2}$
(6) $r_{3} \leq r_{2} \leq r_{1}$

Since usage is a function of age, the expected cost is a function of the four variables $\left(K_{1}, K_{2}, L_{1}, L_{2}\right)$ or $\left(K_{1}, K_{2}, r_{1}, r_{2}\right)$. These decision variables $\left(K_{1}, K_{2}, r_{1}, r_{2}\right)$ are determined by minimizing the expected warranty servicing cost.

Yun and Kang [54] proposed a new warranty servicing strategy which is an extension of the restricted strategy proposed by Iskandar et al. [21]. The warranty region is divided into three subregions $\Omega_{1}, \Omega_{2}$ and $\Omega_{3}$. All repairs in the first and last subregions are minimal. The first repair in the middle subregion is imperfect instead of a replacement and all subsequent repairs in this subregion are minimal (this is analogous to the onedimensional imperfect repair strategy proposed by Yun et al. [55]).

Chukova et al. [13] extended the 3 subregion restricted strategy by Iskandar et al. [21] to an $n$ subregion restricted strategy $\mathcal{S}_{n}$. All product 
failures in the first and last subregions, namely $\Omega_{1}$ and $\Omega_{n}$, are rectified by minimal repair and the first failure in each of the intermediate subregions $\Omega_{i}, i=2,3, \ldots, n-1$, is rectified by replacement and all subsequent failures in the intermediate subregions are rectified by minimal repair. Since the strategy is restricted, the decision variables

$$
\gamma_{n}=\left(K_{1}, K_{2}, \ldots, K_{n-1}, r_{1}\right)
$$

define the shape of the subregions and are determined by minimizing the expected warranty servicing cost.

Having minimal repairs in the first and last subregions are justified because a new product in the first subregion will usually not have any major problems that may cause the manufacturer to replace it and a product in the last subregion is almost out of warranty and the manufacturer can reduce costs by performing minimal repair after each failure in the last subregion until the warranty expires [21].

For more warranty repair-replacement strategies refer to the works by Iskandar et al. [22], Lawless et al. [32], Chun and Tang [15].

In this research, we consider a two-dimensional rectangular warranty region $\Omega=[0, K) \times[0, L)$ which is, as in the paper by Chukova et al. [13], partitioned into $n$ disjoint subregions. The imperfect repair strategy is an extension of the imperfect repair strategy proposed by Yun et al. [55]. The following section provides the details of our imperfect repair strategy.

\subsection{Imperfect Repair Strategy}

We consider a restricted strategy where the warranty region $\Omega$ is partitioned into $n$ disjoint subregions, $\Omega_{i}, i=1,2, \ldots, n$. The warranty region and subregions for the two cases, $r_{1} \leq r_{2}$ and $r_{2} \leq r_{1}$, are depicted in Figure 3.2 and Figure 3.3 respectively.

Since the strategy is restricted, the warranty servicing cost is a function 
of $n$ decision variables

$$
\left(K_{1}, K_{2}, \ldots, K_{n-1}, r_{1}\right)
$$

that uniquely define the subregions $\Omega_{i}, i=1,2, \ldots, n$. The restricted strategy, although less flexible in terms of the shapes of the subregions, is simpler to analyze. Let $C^{\Omega}\left(\psi_{n}\right)$, where

$$
\psi_{n}=\left(K_{1}, K_{2}, \ldots, K_{n-1}, r_{1}\right),
$$

denote the total warranty servicing cost per unit product sold over the $n$-subregion warranty region $\Omega=[0, K) \times[0, L)$. The imperfect repair strategy is such that

- all repairs in the first subregion $\Omega_{1}$ and the last subregion $\Omega_{n}$ are minimal and have constant cost $c_{\text {min }}$;

- the first repair in each of the subregions $\Omega_{i}, i=2,3, \ldots, n-1$ is imperfect and has a cost $c_{i m p}$ which is, in our derivations, assumed to be constant, and all subsequent repairs in each of the subregions are minimal with constant $\operatorname{cost} c_{\min }$; later in the numerical example, we consider costs $c_{i m p}$ that vary with the degrees of the imperfect repairs.

Let $\mathcal{S}_{n}^{\boldsymbol{\delta}}$ denote the imperfect repair strategy, where $n$ is the number of subregions and

$$
\boldsymbol{\delta}=\left(\delta_{1}, \delta_{2}, \ldots, \delta_{n-2}\right)
$$

is the degrees of the imperfect repairs in the $n-2$ intermediate subregions. The objective of this strategy is to minimize the expected total warranty servicing cost per unit product sold by determining the optimal partition $\Omega_{1}, \Omega_{2}, \ldots, \Omega_{n}$, which is equivalent to finding the pairs

$$
\left(K_{1}, L_{1}\right),\left(K_{2}, L_{2}\right), \ldots,\left(K_{n-1}, L_{n-1}\right)
$$


that minimize the expected warranty servicing cost over the entire warranty region. The optimization problem, under the restricted strategy, reduces to finding the optimal decision variables $K_{1}, K_{2}, \ldots, K_{n-1}$ and $r_{1}$ that minimize the expected warranty servicing cost. As in the paper by Chukova et al. [13], let

$$
\psi_{n}^{*}=\left(K_{1}^{*}, K_{2}^{*}, \ldots, K_{n-1}^{*}, r_{1}^{*}\right)
$$

denote the optimal decision variables. Then

$$
\psi_{n}^{*}=\underset{\psi_{n}}{\arg \min } E\left[C^{\Omega}\left(\psi_{n}\right)\right],
$$

where $E\left[C^{\Omega}\left(\psi_{n}\right)\right]$ is the expected total warranty servicing cost per unit product sold.

To model the failures that result in warranty claims and hence, warranty servicing costs, we use the one-dimensional approach to failure modeling, where it is assumed that a linear relationship exists between the age and usage of the product (refer to Chapter 2 for details). In modeling the failures, it is also assumed that

- all failures result in immediate warranty claims;

- all claims are valid;

- the time required to repair or replace the failed product, in comparison to the operating time, is relatively small and can be approximated to zero.

To model imperfect repairs, two approaches are used. The first approach is an adaptation of the age reduction model proposed by Doyen and Gaudoin [16], according to which the imperfect repair reduces the virtual age of the product by an amount proportional to its age immediately prior to the repair. The second approach is a modification of the intensity reduction model proposed by Chukova et al. [11] which suggests that the 
failure rate function of the product after an imperfect repair is between the failure rate of the product immediately after a minimal repair and the failure rate of the product immediately after the previous repair.

The two approaches for modeling imperfect repairs and the associated cost analyses are described in detail in Chapter 4 and Chapter 5. Numerical illustrations of the results derived in both chapters are presented in Chapter 6. 


\section{Chapter 4}

\section{Age Reduction Model}

In this chapter, we discuss the first of the two imperfect repair models, namely the age reduction model, in detail and derive the distribution of the times to imperfect repair and the associated expected total warranty servicing cost for the imperfect repair strategy $\mathcal{S}_{n}^{\boldsymbol{\delta}}$ described in Chapter 3.

\subsection{Model Formulation}

Since we use the one-dimensional approach to modeling failures, according to which

$$
U(t)=R A(t),
$$

where $A(t)$ and $U(t)$ are the age and usage of the product at time $t$, the failure process is a one-dimensional counting process $\{\tilde{N}(t \mid r) ; t \geq 0\}$, conditional on $R=r$, with a predefined initial intensity function. Let the intensity function of the process $\{\tilde{N}(t \mid r) ; t \geq 0\}$ be denoted by $\tilde{\lambda}(t \mid r)$. Since the usage is modeled as a function of the age, the intensity function is a function of the age of the product only [6]. The quantity $\tilde{\lambda}(t \mid r) d t$ can be viewed as the probability that the product currently in use will fail in the small interval $[t, t+d t)$. See Chapter 2 for details.

In the age reduction model, an imperfect repair changes the virtual age 
of the product such that it is less than or equal to the real age of the product. According to the imperfect repair strategy, all repairs in the first and last subregions are minimal, but the first repair in each of the intermediate subregions, $\Omega_{2}, \Omega_{3}, \ldots, \Omega_{n-1}$, is imperfect with a corresponding degree, $\delta_{1}$, $\delta_{2}, \ldots, \delta_{n-2}$. Let $A_{i}(t)$ denote the virtual age at time $t$ following the $i$-th imperfect repair. The virtual age prior to the first imperfect repair is

$$
A_{0}(t)=t
$$

Following the first imperfect repair of degree $\delta_{1}$, there is a reduction, proportional to the degree of repair, in the age, such that

$$
\begin{aligned}
A_{1}(t) & =t-\delta_{1} u_{1} \\
& =A_{0}(t)-\delta_{1} A_{0}\left(u_{1}\right)
\end{aligned}
$$

where $u_{1}$ is the time of the first imperfect repair. The virtual age following the second imperfect repair depends on the degree of the first imperfect repair, $\delta_{1}$, and the degree of the second imperfect repair, $\delta_{2}$, such that

$$
\begin{aligned}
A_{2}(t) & =t-\delta_{1} u_{1}-\delta_{2}\left(u_{2}-\delta_{1} u_{1}\right) \\
& =A_{1}(t)-\delta_{2} A_{1}\left(u_{2}\right) .
\end{aligned}
$$

where $u_{1}$ and $u_{2}$ are the time of the first imperfect repair and the time of the second imperfect repair, respectively. Similarly, after the third imperfect repair at time $u_{3}$, we have

$$
\begin{aligned}
A_{3}(t) & =t-\delta_{1} u_{1}-\delta_{2}\left(u_{2}-\delta_{1} u_{1}\right)-\delta_{3}\left(u_{3}-\delta_{1} u_{1}-\delta_{2}\left(u_{2}-\delta_{1} u_{1}\right)\right) \\
& =A_{2}(t)-\delta_{3} A_{2}\left(u_{3}\right)
\end{aligned}
$$

where $\delta_{3}$ is the degree of the third imperfect repair. In general, the virtual age can be written (recursively) as

$$
A_{i}(t)=A_{i-1}(t)-\delta_{i} A_{i-1}\left(u_{i}\right)
$$

where $\delta_{i}$ is the degree of the $i$-th imperfect repair and $u_{i}$ is the time of the $i$-th imperfect repair [29]. Since all repairs between the imperfect repairs 
are minimal, the intensity function $\tilde{\lambda}(t \mid r)$ of the process after each imperfect repair changes only with respect to the virtual age after the imperfect repairs. Based on the imperfect repair strategy, there can be a maximum of $n-2$ imperfect repairs if there has been at least one failure in each of the subregions $\Omega_{2}, \Omega_{3}, \ldots, \Omega_{n-1}$, since all repairs in the first and last subregions are minimal. Therefore, for any given set of times to imperfect repair $u_{1}, u_{2}, \ldots, u_{n-2}$, the intensity function at time $t$ is given by

$$
\tilde{\lambda}(t \mid r)= \begin{cases}\lambda(t \mid r), & 0 \leq t \leq u_{1} \\ \lambda\left[A_{1}(t) \mid r\right], & u_{1} \leq t \leq u_{2} \\ \lambda\left[A_{2}(t) \mid r\right], & u_{2} \leq t \leq u_{3} \\ \vdots & \\ \lambda\left[A_{i}(t) \mid r\right], & u_{i} \leq t \leq u_{i+1} \\ \vdots & \\ \lambda\left[A_{n-2}(t) \mid r\right], & u_{n-2} \leq t<\infty\end{cases}
$$

See Figure 4.1 for an example of this intensity function.

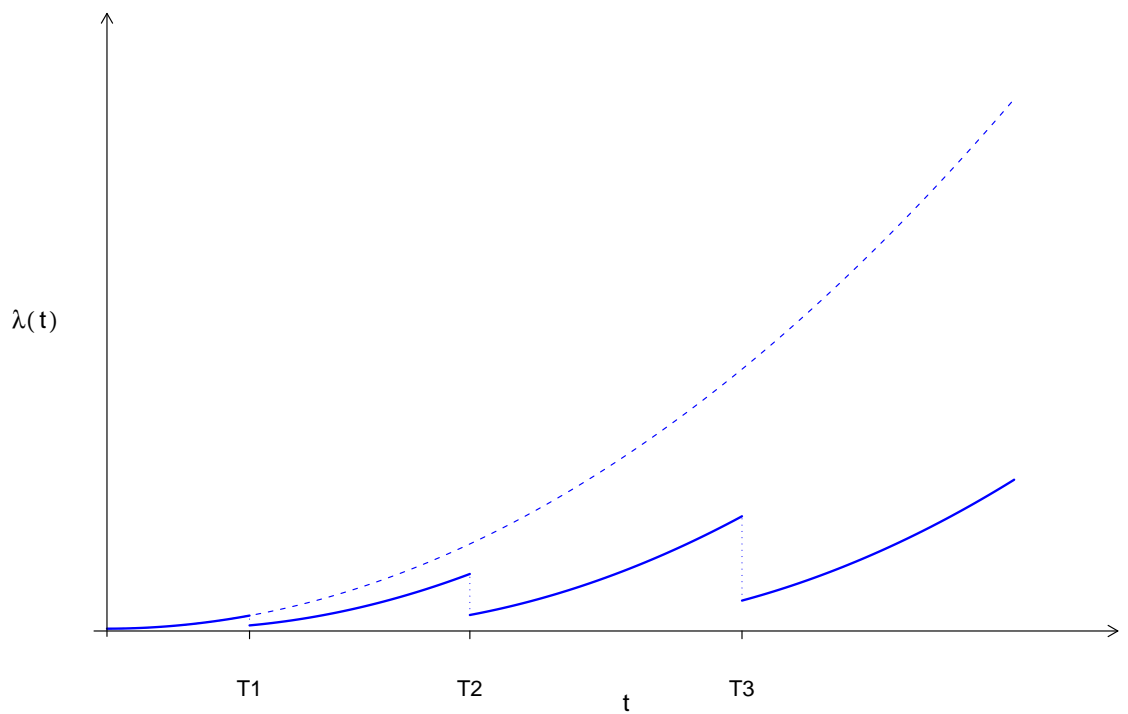

Figure 4.1: Intensity function following imperfect repairs of degree 0.5. 
Since all repairs between the imperfect repairs are minimal, the process between the $i$-th imperfect repair at $u_{i}$ and the next imperfect repair at $u_{i+1}$, for $i=0,1, \ldots, n-2$, can be viewed as a nonhomogeneous Poisson process with intensity function $\lambda\left[A_{i}(t) \mid r\right]$. For $u_{i}<t \leq u_{i+1}$, the expected number of failures in the interval $\left(u_{i}, t\right]$ is given by

$$
\begin{aligned}
E\left[\tilde{N}(t \mid r)-\tilde{N}\left(u_{i} \mid r\right)\right] & =\int_{u_{i}}^{t} \tilde{\lambda}(s \mid r) d s \\
& =\int_{u_{i}}^{t} \lambda\left[A_{i}(s) \mid r\right] d s \\
& =\int_{A_{i}\left(u_{i}\right)}^{A_{i}(t)} \lambda(s \mid r) d s \\
& =\Lambda\left[A_{i}(t) \mid r\right]-\Lambda\left[A_{i}\left(u_{i}\right) \mid r\right],
\end{aligned}
$$

where the virtual age $A_{i}(s)$ after the $i$-th imperfect repair is a function of all previous times to imperfect repair $u_{1}, u_{2}, \ldots, u_{i-1}$, and the $i$-th time to imperfect repair $u_{i}$. The expected number of failures before the first imperfect repair is given by

$$
E[\tilde{N}(t \mid r)]=\Lambda(t \mid r)=\int_{0}^{t} \lambda(s \mid r) d s .
$$

The expected numbers defined in equation (4.3) are conditional on the times to imperfect repair being $u_{1}, u_{2}, \ldots, u_{n-2}$.

In the following section, we derive the density functions of these times to imperfect repair to account for all possible values of $u_{1}, u_{2}, \ldots, u_{n-2}$.

\subsection{Times to Imperfect Repair}

In order to derive the expected total warranty servicing costs, we must first derive the distribution functions of the times to imperfect repair. We 
begin with deriving the distribution function of the time to first failure. As in the paper by Chukova and Johnston [14], let $T_{1 \mid r}$ represent the time to first failure conditional on $R=r$. Then, the distribution function of $T_{1 \mid r}$ is given by

$$
\begin{aligned}
F_{T_{1 \mid r}}(t) & =P\left\{T_{1 \mid r} \leq t\right\} \\
& =1-P\left\{T_{1 \mid r}>t\right\} \\
& =1-P\{\tilde{N}(t \mid r)=0\} \\
& =1-\frac{[\Lambda(t \mid r)]^{0} e^{-\Lambda(t \mid r)}}{0 !} \\
& =1-e^{-\Lambda(t \mid r)},
\end{aligned}
$$

where $\Lambda(t \mid r)$ is defined in equation (4.4). Therefore, the probability that the product does not fail in the interval $[0, t)$, is

$$
\bar{F}_{T_{1 \mid r}}(t)=P\left\{T_{1 \mid r}>t\right\}=P\{\tilde{N}(t \mid r)=0\}=e^{-\Lambda(t \mid r)} .
$$

The density function of $T_{1 \mid r}$ is given by

$$
f_{T_{1 \mid r}}(t)=\frac{d}{d t} F_{T_{1 \mid r}}(t)=\lambda(t \mid r) e^{-\Lambda(t \mid r)} .
$$

Now let the variables $T_{K_{1} \mid r}, T_{K_{2} \mid r}, \ldots, T_{K_{n-2} \mid r}$ each denote the time of the first failure in the corresponding subregion $\Omega_{2}, \Omega_{3}, \ldots, \Omega_{n-1}$. Therefore, $T_{K_{1} \mid r}$ is the time of the first failure after $K_{1}, T_{K_{2} \mid r}$ is the time of the first failure after $K_{2}$ and so on. The distribution function of the time of the first imperfect repair is a conditional form of the distribution of the time to first failure $T_{1 \mid r}$ given in equation (4.5). Hence, for $t>K_{1}$, the distribution function of $T_{K_{1} \mid r}$ is given by

$$
\begin{aligned}
F_{T_{K_{1} \mid r}}(t) & =P\left\{T_{K_{1} \mid r} \leq t\right\} \\
& =1-P\left\{T_{K_{1} \mid r}>t\right\} \\
& =1-P\left\{\tilde{N}(t \mid r)-\tilde{N}\left(K_{1} \mid r\right)=0\right\} \\
& =1-\frac{\left[\Lambda(t \mid r)-\Lambda\left(K_{1} \mid r\right)\right]^{0} e^{-\left[\Lambda(t \mid r)-\Lambda\left(K_{1} \mid r\right)\right]}}{0 !} \\
& =1-e^{-\left[\Lambda(t \mid r)-\Lambda\left(K_{1} \mid r\right)\right]},
\end{aligned}
$$


where $\lambda(s \mid r)$ (equation (4.2)) is the intensity function prior to the first imperfect repair and $\Lambda(s \mid r)$ (equation (4.4)) is the corresponding cumulative intensity function $[14,21]$. The density function for $T_{K_{1} \mid r}$ is given by

$$
f_{T_{K_{1} \mid r}}(t)=\frac{d}{d t} F_{T_{K_{1} \mid r}}(t)=\lambda(t \mid r) e^{-\left[\Lambda(t \mid r)-\Lambda\left(K_{1} \mid r\right)\right]},
$$

and the probability that the first failure after $K_{1}$ is after time $t>K_{1}$ is $\bar{F}_{T_{K_{1} \mid r}}(t)=P\left\{T_{K_{1} \mid r}>t\right\}=P\left\{\tilde{N}(t \mid r)-\tilde{N}\left(K_{1} \mid r\right)=0\right\}=e^{-\left[\Lambda(t \mid r)-\Lambda\left(K_{1} \mid r\right)\right]}$.

The distribution of the time to first failure after $K_{2}, T_{K_{2} \mid r}$, depends on $T_{K_{1} \mid r}$, since the virtual age changes after an imperfect repair. If there have been no failures in the interval $\left(K_{1}, K_{2}\right]$, the distribution of $T_{K_{2} \mid r}$ is similar to that of $T_{K_{1} \mid r}$ derived in equation (4.6), however, if there have been any failures in $\left(K_{1}, K_{2}\right]$, then the virtual age of the product is altered. Hence, the distribution function of $T_{K_{2} \mid r}$ is

$$
\begin{aligned}
F_{T_{K_{2} \mid r}}(t)= & P\left\{T_{K_{2} \mid r} \leq t\right\} \\
= & P\left\{T_{K_{2} \mid r} \leq t, T_{K_{1} \mid r} \leq K_{2}\right\}+P\left\{T_{K_{2} \mid r} \leq t, T_{K_{1} \mid r}>K_{2}\right\} \\
= & P\left\{T_{K_{2} \mid r} \leq t \mid T_{K_{1} \mid r} \leq K_{2}\right\} P\left\{T_{K_{1} \mid r} \leq K_{2}\right\}+P\left\{K_{2}<T_{K_{1} \mid r} \leq t\right\} \\
= & \int_{K_{1}}^{K_{2}} P\left\{T_{K_{2} \mid r} \leq t \mid T_{K_{1} \mid r}=u_{1}\right\} f_{T_{K_{1} \mid r}}\left(u_{1}\right) d u_{1}+\int_{K_{2}}^{t} f_{T_{K_{1} \mid r}}\left(u_{1}\right) d u_{1} \\
& \int_{K_{1}}^{K_{2}}\left(1-e^{-\left\{\Lambda\left[A_{1}(t) \mid r\right]-\Lambda\left[A_{1}\left(K_{2}\right) \mid r\right]\right\}}\right) f_{T_{K_{1} \mid r}}\left(u_{1}\right) d u_{1}+e^{-\left[\Lambda\left(K_{2} \mid r\right)-\Lambda\left(K_{1} \mid r\right)\right]} \\
& \quad-e^{-\left[\Lambda(t \mid r)-\Lambda\left(K_{1} \mid r\right)\right]},
\end{aligned}
$$

where $f_{T_{K_{1} \mid r}}\left(u_{1}\right)$ is given in equation (4.7), and since, conditional on the time of the first imperfect repair being $u_{1}, K_{1}<u_{1}<K_{2}$, we have

$$
\begin{aligned}
P\left\{T_{K_{2} \mid r} \leq t \mid T_{K_{1} \mid r}=u_{1}\right\} & =1-P\left\{\tilde{N}(t \mid r)-\tilde{N}\left(K_{2} \mid r\right)=0\right\} \\
& =1-e^{-\left\{\Lambda\left[A_{1}(t) \mid r\right]-\Lambda\left[A_{1}\left(K_{2}\right) \mid r\right]\right\}} \\
& =1-\exp \left\{-\int_{K_{2}}^{t} \lambda\left[A_{1}(s) \mid r\right] d s\right\} .
\end{aligned}
$$


The probability that the first failure after $K_{2}$ is after time $t>K_{2}$ is given by

$$
\begin{aligned}
\bar{F}_{T_{K_{2} \mid r}}(t) & =P\left\{T_{K_{2} \mid r}>t\right\} \\
& =P\left\{T_{K_{2} \mid r}>t, T_{K_{1} \mid r} \leq K_{2}\right\}+P\left\{T_{K_{2} \mid r}>t, T_{K_{1} \mid r}>K_{2}\right\} \\
& =P\left\{T_{K_{2} \mid r}>t \mid T_{K_{1} \mid r} \leq K_{2}\right\} P\left\{T_{K_{1} \mid r} \leq K_{2}\right\}+P\left\{T_{K_{1} \mid r}>t\right\} \\
& =\int_{K_{1}}^{K_{2}} P\left\{T_{K_{2} \mid r}>t \mid T_{K_{1} \mid r}=u_{1}\right\} f_{T_{K_{1} \mid r}}\left(u_{1}\right) d u_{1}+\bar{F}_{T_{K_{1} \mid r}}(t) \\
& =\int_{K_{1}}^{K_{2}} e^{-\left\{\Lambda\left[A_{1}(t) \mid r\right]-\Lambda\left[A_{1}\left(K_{2}\right) \mid r\right]\right\}} f_{T_{K_{1} \mid r}}\left(u_{1}\right) d u_{1}+e^{-\left[\Lambda(t \mid r)-\Lambda\left(K_{1} \mid r\right)\right]} .
\end{aligned}
$$

For $t>K_{2}$, the density function of the time to first failure after $K_{2}, T_{K_{2} \mid r}$, is given by

$$
\begin{aligned}
f_{T_{K_{2} \mid r}}(t) & =\frac{d}{d t} F_{T_{K_{2} \mid r}}(t) \\
& =\int_{K_{1}}^{K_{2}} \lambda\left[A_{1}(t) \mid r\right] e^{-\left\{\Lambda\left[A_{1}(t) \mid r\right]-\Lambda\left[A_{1}\left(K_{2}\right) \mid r\right]\right\}} f_{T_{K_{1} \mid r}}\left(u_{1}\right) d u_{1}+f_{T_{K_{1} \mid r}}(t)
\end{aligned}
$$

where $f_{T_{K_{1} \mid r}}(t)$ is defined in equation (4.7). These results are a generalization of those derived by Chukova et al. [13]. When instead of an imperfect repair at $u_{1}$ we have a replacement, i.e. when $\delta_{1}=1$, we get

$$
\begin{aligned}
f_{T_{K_{2} \mid r}}(t)= & \int_{K_{1}}^{K_{2}} \lambda\left(t-u_{1} \mid r\right) e^{-\left\{\Lambda\left(t-u_{1} \mid r\right)-\Lambda\left(K_{2}-u_{1} \mid r\right)\right\}} f_{T_{K_{1} \mid r}}\left(u_{1}\right) d u_{1} \\
& \quad+f_{T_{K_{1} \mid r}}(t)
\end{aligned}
$$

which is the density function for the time to first imperfect repair after $K_{2}$ derived by Chukova et al. [13].

In deriving the distribution function of the time to first failure after $K_{3}$, $T_{K_{3} \mid r}$, we must consider the failures in subregions $\Omega_{2}$ and $\Omega_{3}$. For $t>K_{3}$, 
the distribution function of $T_{K_{3} \mid r}$ is given by

$$
\begin{aligned}
& F_{T_{K_{3} \mid r}}(t)=P\left\{T_{K_{3} \mid r} \leq t\right\} \\
& =P\left\{T_{K_{3} \mid r} \leq t, T_{K_{2} \mid r} \leq K_{3}, T_{K_{1} \mid r} \leq K_{2}\right\} \\
& +P\left\{T_{K_{3} \mid r} \leq t, T_{K_{2} \mid r} \leq K_{3}, T_{K_{1} \mid r}>K_{2}\right\} \\
& +P\left\{T_{K_{3} \mid r} \leq t, T_{K_{2} \mid r}>K_{3}, T_{K_{1} \mid r} \leq K_{2}\right\} \\
& +P\left\{T_{K_{3} \mid r} \leq t, T_{K_{2} \mid r}>K_{3}, T_{K_{1} \mid r}>K_{2}\right\} \\
& =P\left\{T_{K_{3} \mid r} \leq t \mid T_{K_{2} \mid r} \leq K_{3}, T_{K_{1} \mid r} \leq K_{2}\right\} P\left\{T_{K_{2} \mid r} \leq K_{3}, T_{K_{1} \mid r} \leq K_{2}\right\} \\
& +P\left\{T_{K_{3} \mid r} \leq t \mid T_{K_{2} \mid r} \leq K_{3}, T_{K_{1} \mid r}>K_{2}\right\} P\left\{T_{K_{2} \mid r} \leq K_{3}, T_{K_{1} \mid r}>K_{2}\right\} \\
& +P\left\{T_{K_{3} \mid r} \leq t \mid T_{K_{2} \mid r}>K_{3}, T_{K_{1} \mid r} \leq K_{2}\right\} P\left\{T_{K_{2} \mid r}>K_{3}, T_{K_{1} \mid r} \leq K_{2}\right\} \\
& +P\left\{T_{K_{3} \mid r} \leq t \mid T_{K_{2} \mid r}>K_{3}, T_{K_{1} \mid r}>K_{2}\right\} P\left\{T_{K_{2} \mid r}>K_{3}, T_{K_{1} \mid r}>K_{2}\right\} \\
& =\int_{K_{2}}^{K_{3}} \int_{K_{1}}^{K_{2}}\left\{\left(1-e^{-\left\{\Lambda\left[A_{2}(t) \mid r\right]-\Lambda\left[A_{2}\left(K_{3}\right) \mid r\right]\right\}}\right)\right. \\
& \left.\times \lambda\left[A_{1}\left(u_{2}\right) \mid r\right] e^{-\left\{\Lambda\left[A_{1}\left(u_{2}\right) \mid r\right]-\Lambda\left[A_{1}\left(K_{2}\right) \mid r\right]\right\}} f_{T_{K_{1} \mid r}}\left(u_{1}\right)\right\} d u_{1} d u_{2} \\
& +\int_{K_{2}}^{K_{3}}\left(1-e^{-\left\{\Lambda\left[A_{1}(t) \mid r\right]-\Lambda\left[A_{1}\left(K_{3}\right) \mid r\right]\right\}}\right) f_{T_{K_{1} \mid r}}\left(u_{1}\right) d u_{1} \\
& +\int_{K_{1}}^{K_{2}}\left\{\left(1-e^{-\left\{\Lambda\left[A_{1}(t) \mid r\right]-\Lambda\left[A_{1}\left(K_{3}\right) \mid r\right]\right\}}\right) e^{-\left\{\Lambda\left[A_{1}\left(K_{3}\right) \mid r\right]-\Lambda\left[A_{1}\left(K_{2}\right) \mid r\right]\right\}}\right. \\
& \left.\times f_{T_{K_{1} \mid r}}\left(u_{1}\right)\right\} d u_{1} \\
& +\left(1-e^{\left\{\Lambda(t \mid r)-\Lambda\left(K_{3} \mid r\right)\right\}}\right) e^{-\left\{\Lambda\left(K_{3} \mid r\right)-\Lambda\left(K_{1} \mid r\right)\right\}} .
\end{aligned}
$$

The first summand of the above density represents the case where there has been at least one failure in each of the two intervals $\left(K_{1}, K_{2}\right]$ and $\left(K_{2}, K_{3}\right]$, i.e. there have been two imperfect repairs prior to $K_{3}$. The second and third summands are the two cases where there has been only one imperfect repair prior to $K_{3}$; in the second summand, no failures have occurred in $\left(K_{1}, K_{2}\right]$ and hence, the first failure after $K_{1}$ is after $K_{2}$, and in 
the third summand, no failures have occurred in $\left(K_{2}, K_{3}\right]$. The last summand is the case where no failures have occurred in $\left(K_{1}, K_{3}\right]$. The density function for $T_{K_{3} \mid r}$ is given by

$$
\begin{aligned}
f_{T_{K_{3} \mid r}}(t)= & \frac{d}{d t} F_{T_{K_{3} \mid r}}(t) \\
= & \int_{K_{2}}^{K_{3}} \int_{K_{1}}^{K_{2}}\left\{\lambda\left[A_{2}(t) \mid r\right] e^{-\left\{\Lambda\left[A_{2}(t) \mid r\right]-\Lambda\left[A_{2}\left(K_{3}\right) \mid r\right]\right\}}\right. \\
& \left.\times \lambda\left[A_{1}\left(u_{2}\right) \mid r\right] e^{-\left\{\Lambda\left[A_{1}\left(u_{2}\right) \mid r\right]-\Lambda\left[A_{1}\left(K_{2}\right) \mid r\right]\right\}} f_{T_{K_{1} \mid r}}\left(u_{1}\right)\right\} d u_{1} d u_{2} \\
& +\int_{K_{2}}^{K_{3}} \lambda\left[A_{1}(t) \mid r\right] e^{-\left\{\Lambda\left[A_{1}(t) \mid r\right]-\Lambda\left[A_{1}\left(K_{3}\right) \mid r\right]\right\}} f_{T_{K_{1} \mid r}}\left(u_{1}\right) d u_{1} \\
& +\int_{K_{1}}^{K_{2}} \lambda\left[A_{1}(t) \mid r\right] e^{-\left\{\Lambda\left[A_{1}(t) \mid r\right]-\Lambda\left[A_{1}\left(K_{2}\right) \mid r\right]\right\}} f_{T_{K_{1} \mid r}}\left(u_{1}\right) d u_{1} \\
& +f_{T_{K_{1} \mid r}}(t) .
\end{aligned}
$$

In the third summand, the exponent term implies that no failures have occurred in the interval $\left(K_{2}, t\right]$, i.e. there have been no imperfect repairs in the previous subinterval $\left(K_{2}, K_{3}\right]$. As mentioned earlier, these results are generalizations of those of Chukova et al. [13]. When $\delta_{1}=\delta_{2}=1$, we get

$$
\begin{aligned}
f_{T_{K_{3}} \mid r}(t)= & \int_{K_{2}}^{K_{3}} \int_{K_{1}}^{K_{2}}\left\{\lambda\left(t-u_{2} \mid r\right) e^{-\left\{\Lambda\left(t-u_{2} \mid r\right)-\Lambda\left(K_{3}-u_{2} \mid r\right)\right\}}\right. \\
& \left.\times \lambda\left(u_{2}-u_{1} \mid r\right) e^{-\left\{\Lambda\left(u_{2}-u_{1} \mid r\right)-\Lambda\left(K_{2}-u_{1} \mid r\right)\right\}} f_{T_{K_{1} \mid r}}\left(u_{1}\right)\right\} d u_{1} d u_{2} \\
& +\int_{K_{2}}^{K_{3}} \lambda\left(t-u_{1} \mid r\right) e^{-\left\{\Lambda\left(t-u_{1} \mid r\right)-\Lambda\left(K_{3}-u_{1} \mid r\right)\right\}} f_{T_{K_{1} \mid r}}\left(u_{1}\right) d u_{1} \\
& +\int_{K_{1}}^{K_{2}} \lambda\left(t-u_{1} \mid r\right) e^{-\left\{\Lambda\left(t-u_{1} \mid r\right)-\Lambda\left(K_{2}-u_{1} \mid r\right)\right\}} f_{T_{K_{1} \mid r}}\left(u_{1}\right) d u_{1} \\
& +f_{T_{K_{1} \mid r}}(t) .
\end{aligned}
$$


The first and second summands correspond to replacements in the third subinterval $\left(K_{2}, K_{3}\right]$. Also, notice that the first element in the first summand is now only a function of the replacement in $\left(K_{2}, K_{3}\right]$. We use the dummy variable $u_{1}$ for the replacement in $\left(K_{1}, K_{2}\right]$, and $u_{2}$ for the replacement in $\left(K_{2}, K_{3}\right]$. The above equation can now be rewritten as

$$
\begin{aligned}
f_{T_{K_{3} \mid r}}(t)= & \int_{K_{2}}^{K_{3}} \lambda\left(t-u_{2} \mid r\right) e^{-\left\{\Lambda\left(t-u_{2} \mid r\right)-\Lambda\left(K_{3}-u_{2} \mid r\right)\right\}} \\
& \times\left\{\int_{K_{1}}^{K_{2}} \lambda\left(u_{2}-u_{1} \mid r\right) e^{-\left\{\Lambda\left(u_{2}-u_{1} \mid r\right)-\Lambda\left(K_{2}-u_{1} \mid r\right)\right\}} f_{T_{K_{1} \mid r}}\left(u_{1}\right) d u_{1}\right. \\
& \left.\quad+f_{T_{K_{1} \mid r}}\left(u_{2}\right)\right\} d u_{2} \\
& +\int_{K_{1}}^{K_{2}} \lambda\left(t-u_{1} \mid r\right) e^{-\left\{\Lambda\left(t-u_{1} \mid r\right)-\Lambda\left(K_{2}-u_{1} \mid r\right)\right\}} f_{T_{K_{1} \mid r}}\left(u_{1}\right) d u_{1} \\
& +f_{T_{K_{1} \mid r}}(t) .
\end{aligned}
$$

Note that the term in the curly brackets is the density function of the time to first failure after $K_{2}$ given in equation (4.8), where $u_{1}$ denotes the last replacement before $K_{2}$. Hence, when $\delta_{1}=\delta_{2}=1$, the above becomes

$$
\begin{aligned}
f_{T_{K_{3} \mid r}}(t)= & \int_{K_{2}}^{K_{3}} \lambda\left(t-u_{2} \mid r\right) e^{-\left\{\Lambda\left(t-u_{2} \mid r\right)-\Lambda\left(K_{3}-u_{2} \mid r\right)\right\}} f_{T_{K_{2} \mid r}}\left(u_{2}\right) d u_{2} \\
& +\int_{K_{1}}^{K_{2}} \lambda\left(t-u_{1} \mid r\right) e^{-\left\{\Lambda\left(t-u_{1} \mid r\right)-\Lambda\left(K_{2}-u_{1} \mid r\right)\right\}} f_{T_{K_{1} \mid r}}\left(u_{1}\right) d u_{1}+f_{T_{K_{1} \mid r}}(t) \\
= & \sum_{j=2}^{3} \int_{K_{j-1}}^{K_{j}} \lambda\left(t-u_{j-1} \mid r\right) e^{-\left\{\Lambda\left(t-u_{j-1} \mid r\right)-\Lambda\left(K_{j}-u_{j-1} \mid r\right)\right\}} f_{T_{K_{j-1} \mid r}}\left(u_{j-1}\right) d u_{j-1} \\
& +f_{T_{K_{1} \mid r}}(t),
\end{aligned}
$$

where $u_{j-1}$ is now the time of the last replacement before $K_{3}$. 
These results match those derived by Chukova et al. [13].

Next, the density function for the time to first failure after $K_{4}, T_{K_{4} \mid r}$, is

$$
\begin{aligned}
f_{T_{K_{4}} \mid r}(t)= & \int_{K_{3}}^{K_{4}} \int_{K_{2}}^{K_{3}} \int_{K_{1}}^{K_{2}}\left\{\lambda\left[A_{3}(t) \mid r\right] e^{-\left\{\Lambda\left[A_{3}(t) \mid r\right]-\Lambda\left[A_{3}\left(K_{4}\right) \mid r\right]\right\}}\right. \\
& \times \lambda\left[A_{2}\left(u_{3}\right) \mid r\right] e^{-\left\{\Lambda\left[A_{2}\left(u_{3}\right) \mid r\right]-\Lambda\left[A_{2}\left(K_{3}\right) \mid r\right]\right\}} \\
& \left.\times \lambda\left[A_{1}\left(u_{2}\right) \mid r\right] e^{-\left\{\Lambda\left[A_{1}\left(u_{2}\right) \mid r\right]-\Lambda\left[A_{1}\left(K_{2}\right) \mid r\right]\right\}} f_{T_{K_{1} \mid r}}\left(u_{1}\right)\right\} d u_{1} d u_{2} d u_{3} \\
& +\int_{K_{2}}^{K_{3}} \int_{K_{1}}^{K_{2}}\left\{\lambda\left[A_{2}(t) \mid r\right] e^{-\left\{\Lambda\left[A_{2}(t) \mid r\right]-\Lambda\left[A_{2}\left(K_{3}\right) \mid r\right]\right\}}\right. \\
& \left.\times \lambda\left[A_{1}\left(u_{2}\right) \mid r\right] e^{-\left\{\Lambda\left[A_{1}\left(u_{2}\right) \mid r\right]-\Lambda\left[A_{1}\left(K_{2}\right) \mid r\right]\right\}} f_{T_{K_{1} \mid r}}\left(u_{1}\right)\right\} d u_{1} d u_{2} \\
& +\int_{K_{3}}^{K_{4}} \int_{K_{2}}^{K_{3}}\left\{\lambda\left[A_{2}(t) \mid r\right] e^{-\left\{\Lambda\left[A_{2}(t) \mid r\right]-\Lambda\left[A_{2}\left(K_{4}\right) \mid r\right]\right\}}\right. \\
& \left.\times{ } \lambda\left[A_{1}\left(u_{2}\right) \mid r\right] e^{-\left\{\Lambda\left[A_{1}\left(u_{2}\right) \mid r\right]-\Lambda\left[A_{1}\left(K_{3}\right) \mid r\right]\right\}} f_{T_{K_{1} \mid r}}\left(u_{1}\right)\right\} d u_{1} d u_{2} \\
& +f_{K_{1}} f_{K_{1} \mid r}(t) . \\
& +\int_{K_{3}}^{K_{4}} \int_{K_{1}}^{K_{2}}\left\{\lambda\left[A_{2}(t) \mid r\right] e^{-\left\{\Lambda\left[A_{2}(t) \mid r\right]-\Lambda\left[A_{2}\left(K_{4}\right) \mid r\right]\right\}}\right. \\
& +\int_{K_{2}}{ }^{K_{2}} \lambda\left[A_{1}(t) \mid r\right] e^{-\left\{\Lambda\left[A_{1}(t) \mid r\right]-\Lambda\left[A_{1}\left(K_{3}\right) \mid r\right]\right\}} f_{T_{K_{1} \mid r}}\left(u_{1}\right) d u_{1} \\
& +\int_{K_{3}}^{K_{3}} \lambda\left[A_{1}(t) \mid r\right] e^{-\left\{\Lambda\left[A_{1}(t) \mid r\right]-\Lambda\left[A_{1}\left(K_{4}\right) \mid r\right]\right\}} f_{T_{K_{1} \mid r}}\left(u_{1}\right) d u_{1} \\
& \left.\left.\left.K_{4}\right) \mid r\right] e^{-\left\{\Lambda\left[A_{1}\left(u_{2}\right) \mid r\right]-\Lambda\left[A_{1}\left(K_{2}\right) \mid r\right]\right\}} f_{T_{K_{1} \mid r}}\left(u_{1}\right)\right\} d u_{1} d u_{2}
\end{aligned}
$$


The density function for $T_{K_{4} \mid r}$ is derived in a manner similar to $f_{T_{K_{3} \mid r}}(t)$, and hence, we have omitted the derivation process. The summands in the density function denote the cases where none, one, two or three imperfect repairs have been performed before $K_{4}$. The first summand is the case where there has been at least one failure (and therefore, one imperfect repair) in each of the subintervals $\left(K_{1}, K_{2}\right],\left(K_{2}, K_{3}\right]$, and $\left(K_{3}, K_{4}\right]$ before $K_{4}$. The next three summands each represents the case where there has been at least one failure in each of the two corresponding subintervals and the next three summands each represents the case where there has been at least one failure in the corresponding subinterval. The last summand represents the case where no failures have occurred in the subinterval $\left(K_{1}, K_{4}\right]$.

When $\delta_{1}=\delta_{2}=\delta_{3}=1$, as shown for $f_{T_{K_{3} \mid r}}(t)$, this density function collapses to

$$
\begin{aligned}
f_{T_{K_{4} \mid r}}(t)= & \sum_{j=2}^{4} \int_{K_{j-1}}^{K_{j}} \lambda\left(t-u_{j-1} \mid r\right) e^{-\left\{\Lambda\left(t-u_{j-1} \mid r\right)-\Lambda\left(K_{j}-u_{j-1} \mid r\right)\right\}} f_{T_{K_{j-1} \mid r}}\left(u_{j-1}\right) d u_{j-1} \\
& +f_{T_{K_{1} \mid r}}(t)
\end{aligned}
$$

where $u_{j-1}$ is the time of the last replacement before $K_{4}$. Again, this matches the results derived by Chukova et al. [13].

In general, to derive the density function of the first repair after $K_{l}$, i.e. the time $T_{K_{l \mid r}}$ of the $l$-th imperfect repair, where $l=1,2, \ldots, n-2$, we need to consider all possible combinations of imperfect repairs in the previous subregions $\Omega_{2}, \Omega_{3}, \ldots, \Omega_{l}$. Let $i$ denote the number of possible previous imperfect repairs. For $T_{K_{l \mid r}}$, the time of the first failure after $K_{l}$, we have $i=0,1, \ldots, l-1$ possible previous imperfect repairs. When $i=0$, the time of the first failure after $K_{l}, T_{K_{l \mid r}}$, is the time of the first failure after $K_{1}$. In other words, no failures have occurred in the interval $\left(K_{1}, K_{l}\right]$ and the case is straight-forward. When $i=1$, there has been one imperfect repair before $K_{l}$, which could be in any one of the subintervals $\left(K_{1}, K_{2}\right],\left(K_{2}, K_{3}\right], \ldots,\left(K_{l-1}, K_{l}\right]$. When $i=2$, there have been two imperfect repairs before $K_{l}$, which could be in any two of the subintervals 
$\left(K_{1}, K_{2}\right],\left(K_{2}, K_{3}\right], \ldots,\left(K_{l-1}, K_{l}\right]$, and so on. By conditioning on the variables $u_{1}, u_{2}, \ldots, u_{l-1}$ and then removing the condition, we account for all possible times to imperfect repair. Following the patterns in the density functions derived earlier for $T_{K_{1} \mid r}, T_{K_{2} \mid r}, T_{K_{3} \mid r}$ and $T_{K_{4} \mid r}$, for a given $i>0$ and a given $l$, we define the sum

$$
\begin{aligned}
\sum_{\forall\left\{j_{1}, j_{2} \ldots, j_{i}\right\} \in J_{i, l}} \int_{K_{j_{i}-1}}^{K_{j_{i}}} \ldots \int_{K_{j_{2}-1}}^{K_{j_{2}}} \int_{K_{j_{1}-1}}^{K_{j_{1}}}\left\{\lambda\left[A_{i}(t) \mid r\right] e^{-\left\{\Lambda\left[A_{i}(t) \mid r\right]-\Lambda\left[A_{i}\left(K_{j_{i}}\right)\right]\right\}}\right. \\
\times \lambda\left[A_{i-1}\left(u_{i}\right) \mid r\right] e^{-\left\{\Lambda\left[A_{i-1}\left(u_{i}\right) \mid r\right]-\Lambda\left[A_{i-1}\left(K_{j_{i-1}}\right)\right]\right\}} \\
\quad \vdots \\
\times \lambda\left[A_{1}\left(u_{2}\right) \mid r\right] e^{-\left\{\Lambda\left[A_{1}\left(u_{2}\right) \mid r\right]-\Lambda\left[A_{1}\left(K_{j_{1}}\right)\right]\right\}} \\
\times f_{\left.T_{K_{1} \mid r}\left(u_{1}\right)\right\} d u_{1} d u_{2} \ldots d u_{i}}
\end{aligned}
$$

which represents the density corresponding to all possible combinations of the $i$ imperfect repairs before $K_{l}$. For $i>0$, the set

$$
J_{i, l}=\left\{\left\{j_{1}, \ldots, j_{i}\right\}:\left\{j_{1}, \ldots, j_{i}\right\} \subseteq\{2, \ldots, l\} \text { and } j_{1}<\cdots<j_{i}\right\}
$$

provides all possible combinations of $\left\{K_{j_{1}}, \ldots, K_{j_{i}}\right\}$ which generate the subintervals in which the imperfect repairs have been performed [12]. Therefore, the set $J_{i, l}$ works such that when $i=1$, we have

$$
\begin{aligned}
& \sum_{\forall\left\{j_{1}\right\} \in J_{1, l} K_{j_{1}-1}} \int_{j_{1}}^{K_{j_{1}}} \lambda\left[A_{1}(t) \mid r\right] e^{-\left\{\Lambda\left[A_{1}(t) \mid r\right]-\Lambda\left[A_{1}\left(K_{j_{1}}\right)\right]\right\}} f_{T_{K_{1} \mid r}}\left(u_{1}\right) d u_{1} \\
= & \sum_{K_{j_{1}-1}}^{l} \int_{K_{j_{1}}}^{K_{1}} \lambda\left[A_{1}(t) \mid r\right] e^{-\left\{\Lambda\left[A_{1}(t) \mid r\right]-\Lambda\left[A_{1}\left(K_{j_{1}}\right)\right]\right\}} f_{T_{K_{1} \mid r}}\left(u_{1}\right) d u_{1},
\end{aligned}
$$

which implies that when $i=1$, there has been one imperfect repair before $K_{l}$, which could be in any one of the subintervals $\left(K_{1}, K_{2}\right],\left(K_{2}, K_{3}\right], \ldots$, $\left(K_{l-1}, K_{l}\right]$. 
When $i=2$, we have

$$
\begin{aligned}
\sum_{\forall\left\{j_{1}, j_{2}\right\} \in J_{2, l} \int_{K_{j_{2}-1}}^{K_{j_{2}}} \int_{K_{j_{1}-1}}^{K_{j_{1}}}\{\lambda} & {\left[A_{2}(t) \mid r\right] e^{-\left\{\Lambda\left[A_{2}(t) \mid r\right]-\Lambda\left[A_{2}\left(K_{j_{2}}\right)\right]\right\}} } \\
\times & \lambda\left[A_{1}\left(u_{2}\right) \mid r\right] e^{-\left\{\Lambda\left[A_{1}\left(u_{2}\right) \mid r\right]-\Lambda\left[A_{1}\left(K_{j_{1}}\right)\right]\right\}} \\
& \left.\times f_{T_{K_{1} \mid r}}\left(u_{1}\right)\right\} d u_{1} d u_{2}
\end{aligned}
$$

which implies that when $i=2$, there have been two imperfect repairs before $K_{l}$ which could be in any two of the subintervals $\left(K_{1}, K_{2}\right],\left(K_{2}, K_{3}\right]$, $\ldots,\left(K_{l-1}, K_{l}\right]$, and so on. When $i=l-1$, we have the term

$$
\begin{aligned}
& \int_{K_{l-1}}^{K_{l}} \int_{K_{l-2}}^{K_{l-1}} \ldots \int_{K_{2}}^{K_{3}} \int_{K_{1}}^{K_{2}}\left\{\lambda\left[A_{l-1}(t) \mid r\right] e^{-\left\{\Lambda\left[A_{l-1}(t) \mid r\right]-\Lambda\left[A_{l-1}\left(K_{l}\right)\right]\right\}}\right. \\
& \quad \times \lambda\left[A_{l-2}\left(u_{l-1}\right) \mid r\right] e^{-\left\{\Lambda\left[A_{l-2}\left(u_{l-1}\right) \mid r\right]-\Lambda\left[A_{l-2}\left(K_{l-1}\right)\right]\right\}} \\
& \quad \times \lambda\left[A_{l-3}\left(u_{l-2}\right) \mid r\right] e^{-\left\{\Lambda\left[A_{l-3}\left(u_{l-2}\right) \mid r\right]-\Lambda\left[A_{l-3}\left(K_{l-2}\right)\right]\right\}} \\
& \quad \vdots \\
& \quad \times \quad \lambda\left[A_{1}\left(u_{2}\right) \mid r\right] e^{-\left\{\Lambda\left[A_{1}\left(u_{2}\right) \mid r\right]-\Lambda\left[A_{1}\left(K_{2}\right)\right]\right\}} \\
& \left.\times f_{T_{K_{1} \mid r}}\left(u_{1}\right)\right\} d u_{1} d u_{2} \ldots d u_{l-2} d u_{l-1}
\end{aligned}
$$

which corresponds to the case where at least one failure has occurred in all the subintervals before $K_{l}$, namely $\left(K_{1}, K_{2}\right],\left(K_{2}, K_{3}\right], \ldots,\left(K_{l-1}, K_{l}\right]$. In other words, there has been an imperfect repair in all the subregions $\Omega_{2}, \Omega_{3}, \ldots, \Omega_{l}$ before $K_{l}$.

Since the events $i=0, i=1, \ldots, i=l-1$ are exclusive, the distribution function for $T_{K_{l} \mid r}$ is the sum of all probabilities corresponding to these events. And hence, when $i=0$, the density function component is just $f_{T_{K_{1} \mid r}}(t)$, and when $i>0$, the density function component is the sum over all $i>0$ of the summands defined in equation (4.9), thus accounting for both the number of possible previous imperfect repairs and the combination of intervals in which they've been performed. 
Therefore, density function for $T_{K_{l} \mid r}$ is given by

$$
\begin{aligned}
f_{T_{K_{l} \mid r}}(t)= & f_{T_{K_{1} \mid r}}(t) \\
+ & \sum_{i=1}^{l-1} \sum_{\forall\left\{j_{1}, j_{2} \ldots, j_{i}\right\} \in J_{i, l} K_{K_{i}-1}} \int_{K_{j_{2}-1}}^{K_{j_{i}}} \ldots \int_{K_{j_{1}-1}}^{K_{j_{2}}}\left\{\lambda\left[A_{i}(t) \mid r\right] e^{-\left\{\Lambda\left[A_{i}(t) \mid r\right]-\Lambda\left[A_{i}\left(K_{j_{i}}\right) \mid r\right]\right\}}\right. \\
& \times \lambda\left[A_{i-1}\left(u_{i}\right) \mid r\right] e^{-\left\{\Lambda\left[A_{i-1}\left(u_{i}\right) \mid r\right]-\Lambda\left[A_{i-1}\left(K_{j_{i-1}}\right) \mid r\right]\right\}} \\
& \quad \vdots \\
& \times \lambda\left[A_{1}\left(u_{2}\right) \mid r\right] e^{-\left\{\Lambda\left[A_{1}\left(u_{2}\right) \mid r\right]-\Lambda\left[A_{1}\left(K_{j_{1}}\right) \mid r\right]\right\}} \\
& \left.\times f_{T_{K_{1} \mid r}}\left(u_{1}\right)\right\} d u_{1} d u_{2} \ldots d u_{i}
\end{aligned}
$$

where $A_{i}($.$) is the virtual age after the i$-th imperfect repair given in equation (4.1) and $f_{T_{K_{1} \mid r}}(t)$ is defined in equation (4.7). These results are generalizations of those presented in Chukova et al. [13]. When

$$
\delta_{1}=\delta_{2}=\ldots=\delta_{l-1}=1
$$

for $t>K_{l}$, we have

$$
\begin{aligned}
f_{T_{K_{l} \mid r}}(t) & =f_{T_{K_{1} \mid r}}(t) \\
& +\sum_{j=2}^{l} \int_{K_{j-1}}^{K_{j}} \lambda\left(t-u_{j-1} \mid r\right) e^{-\left\{\Lambda\left(t-u_{j-1} \mid r\right)-\Lambda\left(K_{j}-u_{j-1} \mid r\right)\right\}} f_{T_{K_{j-1} \mid r}}\left(u_{j-1}\right) d u_{j-1},
\end{aligned}
$$

where $u_{j-1}$ is now the time of the replacement in the subinterval $\left[K_{j-1}, K_{j}\right)$ or in $\Omega_{j}$ and may not be the time of the $j$-th replacement; $u_{j-1}$ is the time of the last replacement before $K_{l}$. This matches the results derived by Chukova et al. [13]. We now proceed to deriving the expected total warranty servicing cost per product sold over the warranty region. 


\subsection{Warranty Servicing Costs}

Let $E\left[C^{\Omega}\left(\psi_{n}\right)\right]$ denote the expected total warranty servicing cost over the warranty region $\Omega$, where

$$
\psi_{n}=\left(K_{1}, K_{2}, \ldots, K_{n-1}, r_{1}\right)
$$

Since the imperfect repair strategy is restricted (see Chapter 3 for details), such that

$$
\frac{L_{1}}{K_{1}}=\frac{L_{2}}{K_{2}}=\ldots=\frac{L_{n-1}}{K_{n-1}}=r_{1} \quad \text { and } \quad \frac{L}{K}=r_{2} ，
$$

in determining the expected total warranty servicing cost $E\left[C^{\Omega}\left(\psi_{n}\right)\right]$, we need to consider only two cases: $r_{1} \leq r_{2}$ (Figure 3.2) and $r_{2} \leq r_{1}$ (Figure 3.3). Let $E\left[C_{A}^{\Omega}\left(\psi_{n}\right)\right]$ denote the expected total warranty servicing cost for the first case $r_{1} \leq r_{2}$ and let $E\left[C_{B}^{\Omega}\left(\psi_{n}\right)\right]$ denote the expected total warranty servicing cost for the second case $r_{2} \leq r_{1}[21,13]$. That is,

- Case A: $E\left[C^{\Omega}\left(\psi_{n}\right)\right]=E\left[C_{A}^{\Omega}\left(\psi_{n}\right)\right]$

- Case B: $E\left[C^{\Omega}\left(\psi_{n}\right)\right]=E\left[C_{B}^{\Omega}\left(\psi_{n}\right)\right]$

The main results of this chapter are the density function derived in equation (4.10), and the expected warranty servicing costs $E\left[C_{A}^{\Omega}\left(\psi_{n}\right)\right]$ for Case A and $E\left[C_{B}^{\Omega}\left(\psi_{n}\right)\right]$ for Case $\mathrm{B}$, for the age reduction model, which we derive in the following sections.

\subsubsection{Case A: $r_{1} \leq r_{2}$}

Since we are using the one-dimensional approach where the usage of the product is a function of the age of the product, in order to derive the expected total warranty servicing cost $E\left[C_{A}^{\Omega}\left(\psi_{n}\right)\right]$, we condition on $R=r$. As in the papers by Iskandar et al. [21] and Chukova et al. [13], we subdivide the warranty region based on the rates $r_{1}$ and $r_{2}$, and, conditional on 
the usage rate $R=r$, derive the expected warranty servicing costs for the following three sub-cases:

$$
\begin{array}{ll}
\text { (1) } & r \leq r_{1} \leq r_{2} \\
\text { (2) } & r_{1} \leq r \leq r_{2} \\
\text { (3) } & r_{1} \leq r_{2} \leq r
\end{array}
$$

Let $E\left[C_{r}^{(1)}\left(\psi_{n}\right)\right], E\left[C_{r}^{(2)}\left(\psi_{n}\right)\right]$, and $E\left[C_{r}^{(3)}\left(\psi_{n}\right)\right]$ denote the expected warranty servicing costs, conditional on $R=r$, for the three sub-cases respectively [13]. Then the expected total warranty servicing cost for Case $A$ is given by

$$
E\left[C_{A}^{\Omega}\left(\psi_{n}\right)\right]=\int_{0}^{r_{1}} E\left[C_{r}^{(1)}\left(\psi_{n}\right)\right] d G(r)+\int_{r_{1}}^{r_{2}} E\left[C_{r}^{(2)}\left(\psi_{n}\right)\right] d G(r)+\int_{r_{2}}^{\infty} E\left[C_{r}^{(3)}\left(\psi_{n}\right)\right] d G(r) .
$$

Each of the expected costs $E\left[C_{r}^{(j)}\left(\psi_{n}\right)\right], j=1,2,3$, are the sum of the expected costs in each of the subregions $\Omega_{1}, \Omega_{2}, \ldots, \Omega_{n}$, which are denoted by

$$
E\left[C_{r}^{\Omega_{1}}\left(\psi_{n}\right)\right], E\left[C_{r}^{\Omega_{2}}\left(\psi_{n}\right)\right], \ldots, E\left[C_{r}^{\Omega_{n}}\left(\psi_{n}\right)\right] .
$$

That is, the expected warranty servicing cost conditional on $R=r$, for the three sub-cases, $j=1,2,3$, is given by

$$
E\left[C_{r}^{(j)}\left(\psi_{n}\right)=E\left[C_{r}^{\Omega_{1}}\left(\psi_{n}\right)\right]+E\left[C_{r}^{\Omega_{2}}\left(\psi_{n}\right)\right]+\ldots+E\left[C_{r}^{\Omega_{n}}\left(\psi_{n}\right)\right] .\right.
$$

For simplicity, since it is clear by the context, we omit the usage of the index $j$ on the right hand side of the above equation.

We now proceed to deriving the expected costs $E\left[C_{A}^{\Omega}\left(\psi_{n}\right)\right]$ and $E\left[C_{B}^{\Omega}\left(\psi_{n}\right)\right]$. We will only derive the expected cost for sub-case A-(1), since the expected costs for A-(2) and A-(3) and Case B can be obtained in a similar way, with minor adjustments.

(1) $\mathrm{r} \leq \mathrm{r}_{1} \leq \mathrm{r}_{2}$

Figure 4.2 depicts the case where $r \leq r_{1} \leq r_{2}$. Let $c_{\text {min }}$ and $c_{i m p}$ denote the cost of a minimal repair and the cost of an imperfect repair respectively. 


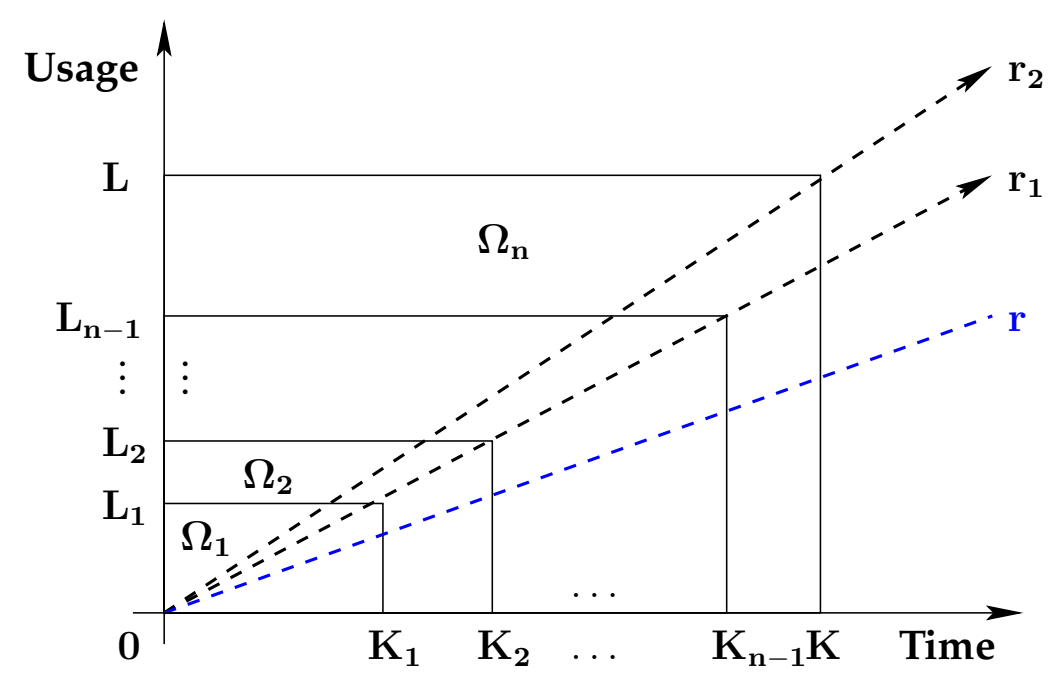

Figure 4.2: $r \leq r_{1} \leq r_{2}$.

The costs $c_{\min }$ and $c_{i m p}$ are assumed to be constant. Later in the numerical example, we adjust the costs $c_{i m p}$ of the imperfect repairs based on their degrees.

When deriving the warranty servicing cost in a subregion, we must consider all imperfect repairs performed in the preceding subregions, since they alter the virtual age of the product. By considering the number of possible imperfect repairs and conditioning on the time of these repairs, we can derive the conditional expected warranty servicing costs in the subregion. Later, we remove the conditioning using the summands of the density function derived earlier to account for all possible combinations of subintervals with these imperfect repairs and all possible repair times.

The expected warranty servicing cost in the first subregion $\Omega_{1}$ is not conditional on times to imperfect repair, since all repairs in this subregion are minimal. Therefore, the warranty servicing cost in this subregion is given by

$$
\begin{cases}c_{\min } \tilde{N}\left(K_{1} \mid r\right), & \tilde{N}\left(K_{1} \mid r\right)>0 \\ 0, & \text { otherwise }\end{cases}
$$


where $\tilde{N}\left(K_{1} \mid r\right)$ is the number of failures in the interval $\left[0, K_{1}\right]$ and the expected warranty servicing cost in $\Omega_{1}$ is given by

$$
\begin{cases}c_{\min } \Lambda\left(K_{1} \mid r\right), & \tilde{N}\left(K_{1} \mid r\right)>0 \\ 0, & \text { otherwise }\end{cases}
$$

where

$$
\Lambda\left(K_{1} \mid r\right)=\int_{0}^{K_{1}} \lambda(s \mid r) d s
$$

Hence, the expected warranty cost in the first subregion $\Omega_{1}$ is

$$
E\left[C_{r}^{\Omega_{1}}\left(\psi_{n}\right)\right]=c_{\min } \Lambda\left(K_{1} \mid r\right)
$$

The first failure in the second subregion is rectified by an imperfect repair and all subsequent failures are rectified by minimal repair. If the first failure after $K_{1}$ occurs at time $u_{1}$, then conditional on $T_{K_{1} \mid r}=u_{1}$, the conditional warranty servicing cost in subregion $\Omega_{2}$ is given by

$$
\begin{cases}c_{i m p}+c_{\min }\left[\tilde{N}\left(K_{2} \mid r\right)-\tilde{N}\left(u_{1} \mid r\right)\right], & u_{1} \leq K_{2} \\ 0, & u_{1}>K_{2}\end{cases}
$$

where $\tilde{N}\left(K_{2} \mid r\right)-\tilde{N}\left(u_{1} \mid r\right)$ is the number of failures, and hence minimal repairs, in the interval $\left(u_{1}, K_{2}\right]$. The conditional expected warranty servicing cost in $\Omega_{2}$ is given by

$$
\begin{cases}c_{i m p}+c_{\min }\left\{\Lambda\left[A_{1}\left(K_{2}\right) \mid r\right]-\Lambda\left[A_{1}\left(u_{1}\right) \mid r\right]\right\}, & u_{1} \leq K_{2} \\ 0, & u_{1}>K_{2}\end{cases}
$$

where $A_{1}(t)$ is the virtual age after the first imperfect repair at time $u_{1}$ with degree $\delta_{1}$. Now removing the conditioning on $T_{K_{1} \mid r}=u_{1}$, we get

$$
\begin{aligned}
E\left[C_{r}^{\Omega_{2}}\left(\psi_{n}\right)\right]=\int_{K_{1}}^{K_{2}}\{ & \left(c_{i m p}+c_{\min }\left\{\Lambda\left[A_{1}\left(K_{2}\right) \mid r\right]-\Lambda\left[A_{1}\left(u_{1}\right) \mid r\right]\right\}\right) \\
& \left.\times f_{T_{K_{1} \mid r}}\left(u_{1}\right)\right\} d u_{1}
\end{aligned}
$$


The first failure in the third subregion $\Omega_{3}$ is also rectified by an imperfect repair and all subsequent failures in this subregion are rectified by minimal repair. In computing the warranty cost in subregion $\Omega_{3}$, we must consider the two cases $T_{K_{1} \mid r} \leq K_{2}$ and $T_{K_{1} \mid r}>K_{2}$. Therefore, conditional on the first failure after $K_{1}$ being at time $u_{1}$, i.e., time of the first imperfect repair being $T_{K_{1} \mid r}=u_{1}$ and the time of the second imperfect repair being $u_{2}$, the conditional warranty servicing cost in subregion $\Omega_{3}$ is given by

$$
\begin{cases}c_{i m p}+c_{\min }\left[\tilde{N}\left(K_{3} \mid r\right)-\tilde{N}\left(u_{1} \mid r\right)\right], & K_{2}<u_{1} \leq K_{3} \\ c_{i m p}+c_{\min }\left[\tilde{N}\left(K_{3} \mid r\right)-\tilde{N}\left(u_{2} \mid r\right)\right], & u_{1} \leq K_{2} \text { and } u_{2} \leq K_{3} \\ 0, & \text { otherwise }\end{cases}
$$

where $\tilde{N}\left(K_{3} \mid r\right)-\tilde{N}\left(u_{1} \mid r\right)$ and $\tilde{N}\left(K_{3} \mid r\right)-\tilde{N}\left(u_{2} \mid r\right)$ count the number of minimal repairs in the subintervals $\left(u_{1}, K_{3}\right]$ and $\left(u_{2}, K_{3}\right]$ respectively. The conditional expected warranty servicing cost in subregion $\Omega_{3}$ is

$$
\begin{cases}c_{i m p}+c_{\min }\left\{\Lambda\left[A_{1}\left(K_{3}\right) \mid r\right]-\Lambda\left[A_{1}\left(u_{1}\right) \mid r\right]\right\}, & K_{2}<u_{1} \leq K_{3} \\ c_{i m p}+c_{\min }\left\{\Lambda\left[A_{2}\left(K_{3}\right) \mid r\right]-\Lambda\left[A_{2}\left(u_{2}\right) \mid r\right]\right\}, & u_{1} \leq K_{2} \text { and } u_{2} \leq K_{3} \\ 0, & \text { otherwise }\end{cases}
$$

On removing the conditioning on the time of the first imperfect repair $u_{1}$ and the time of the second imperfect repair $u_{2}$, we get the expected warranty servicing cost in subregion $\Omega_{3}$

$$
\begin{aligned}
E\left[C_{r}^{\Omega_{3}}\left(\psi_{n}\right)\right]= & \int_{K_{2}}^{K_{3}}\left\{\left(c_{i m p}+c_{\min }\left\{\Lambda\left[A_{1}\left(K_{3}\right) \mid r\right]-\Lambda\left[A_{1}\left(u_{1}\right) \mid r\right]\right\}\right)\right. \\
& \left.\times f_{T_{K_{1} \mid r}}\left(u_{1}\right)\right\} d u_{1} \\
+ & \int_{K_{2}}^{K_{3}} \int_{K_{1}}^{K_{2}}\left\{\left(c_{i m p}+c_{\min }\left\{\Lambda\left[A_{2}\left(K_{3}\right) \mid r\right]-\Lambda\left[A_{2}\left(u_{2}\right) \mid r\right]\right\}\right)\right. \\
& \left.\times \lambda\left[A_{1}\left(u_{2}\right) \mid r\right] e^{-\left\{\Lambda\left[A_{1}\left(u_{2}\right) \mid r\right]-\Lambda\left[A_{1}\left(K_{2}\right) \mid r\right]\right\}} f_{T_{K_{1} \mid r}}\left(u_{1}\right)\right\} d u_{1} d u_{2} .
\end{aligned}
$$


Similar to the cost in $\Omega_{3}$, the cost in $\Omega_{4}$ is derived by considering all possible imperfect repairs in previous subregions. Conditional on the time of the first imperfect repair being $u_{1}$, the time of the second imperfect repair being $u_{2}$ and the time of the third imperfect repair being $u_{3}$, we consider three cases: the case where no imperfect repairs have been performed before $\Omega_{4}$ (i.e, before $K_{3}$ ), the case where one imperfect repair has been performed before $\Omega_{4}$, and the case where two imperfect repairs have been performed before $\Omega_{4}$. The first failure in this subregion is followed by an imperfect repair and all subsequent failures are followed by minimal repairs. Hence, the conditional warranty servicing cost in this subregion is given by

$$
\begin{cases}c_{i m p}+c_{\min }\left[\tilde{N}\left(K_{4} \mid r\right)-\tilde{N}\left(u_{1} \mid r\right)\right], & K_{3}<u_{1} \leq K_{4} \\ c_{i m p}+c_{\min }\left[\tilde{N}\left(K_{4} \mid r\right)-\tilde{N}\left(u_{2} \mid r\right)\right], & u_{1} \leq K_{2} \text { and } K_{3}<u_{2} \leq K_{4} \\ c_{i m p}+c_{\min }\left[\tilde{N}\left(K_{4} \mid r\right)-\tilde{N}\left(u_{2} \mid r\right)\right], & K_{2}<u_{1} \leq K_{3} \text { and } K_{3}<u_{2} \leq K_{4} \\ c_{i m p}+c_{\min }\left[\tilde{N}\left(K_{4} \mid r\right)-\tilde{N}\left(u_{3} \mid r\right)\right], & u_{1} \leq K_{2}, u_{2} \leq K_{3} \text { and } u_{3} \leq K_{4} \\ 0, & \text { otherwise }\end{cases}
$$

where the variables denote the number of minimal repairs in the intervals $\left(u_{1}, K_{4}\right],\left(u_{2}, K_{4}\right]$ and $\left(u_{3}, K_{4}\right]$ respectively. The expected conditional warranty servicing cost in this subregion is given by

$$
\begin{cases}c_{i m p}+c_{\min }\left\{\Lambda\left[A_{1}\left(K_{4}\right) \mid r\right]-\Lambda\left[A_{1}\left(u_{1}\right) \mid r\right]\right\}, & K_{3}<u_{1} \leq K_{4} \\ c_{i m p}+c_{\min }\left\{\Lambda\left[A_{2}\left(K_{4}\right) \mid r\right]-\Lambda\left[A_{2}\left(u_{2}\right) \mid r\right]\right\}, & u_{1} \leq K_{2} \text { and } K_{3}<u_{2} \leq K_{4} \\ c_{i m p}+c_{\min }\left\{\Lambda\left[A_{2}\left(K_{4}\right) \mid r\right]-\Lambda\left[A_{2}\left(u_{2}\right) \mid r\right]\right\}, & K_{2}<u_{1} \leq K_{3} \\ & \text { and } K_{3}<u_{2} \leq K_{4} \\ c_{i m p}+c_{\min }\left\{\Lambda\left[A_{3}\left(K_{4}\right) \mid r\right]-\Lambda\left[A_{3}\left(u_{3}\right) \mid r\right]\right\}, & u_{1} \leq K_{2}, u_{2} \leq K_{3} \\ 0, & \text { and } u_{3} \leq K_{4} \\ 0 & \text { otherwise . }\end{cases}
$$

On removing the conditioning on the times to imperfect repair $u_{1}, u_{2}$ and $u_{3}$, we get the expected warranty servicing cost in the fourth subregion, $\Omega_{4}$. 
That is

$$
\begin{aligned}
& E\left[C_{r}^{\Omega_{4}}\left(\psi_{n}\right)\right]=\int_{K_{3}}^{K_{4}}\left\{\left(c_{i m p}+c_{\min }\left\{\Lambda\left[A_{1}\left(K_{4}\right) \mid r\right]-\Lambda\left[A_{1}\left(u_{1}\right) \mid r\right]\right\}\right)\right. \\
& \left.\times f_{T_{K_{1} \mid r}}\left(u_{1}\right)\right\} d u_{1} \\
& +\int_{K_{3}}^{K_{4}} \int_{K_{1}}^{K_{2}}\left\{\left(c_{i m p}+c_{\min }\left\{\Lambda\left[A_{2}\left(K_{4}\right) \mid r\right]-\Lambda\left[A_{2}\left(u_{2}\right) \mid r\right]\right\}\right)\right. \\
& \times \lambda\left[A_{1}\left(u_{2}\right) \mid r\right] e^{-\left\{\Lambda\left[A_{1}\left(u_{2}\right) \mid r\right]-\Lambda\left[A_{1}\left(K_{2}\right) \mid r\right]\right\}} \\
& \left.\times f_{T_{K_{1} \mid r}}\left(u_{1}\right)\right\} d u_{1} d u_{2} \\
& +\int_{K_{3}}^{K_{4}} \int_{K_{2}}^{K_{3}}\left\{\left(c_{i m p}+c_{\min }\left\{\Lambda\left[A_{2}\left(K_{4}\right) \mid r\right]-\Lambda\left[A_{2}\left(u_{2}\right) \mid r\right]\right\}\right)\right. \\
& \times \lambda\left[A_{1}\left(u_{2}\right) \mid r\right] e^{-\left\{\Lambda\left[A_{1}\left(u_{2}\right) \mid r\right]-\Lambda\left[A_{1}\left(K_{3}\right) \mid r\right]\right\}} \\
& \left.\times f_{T_{K_{1} \mid r}}\left(u_{1}\right)\right\} d u_{1} d u_{2} \\
& +\int_{K_{3}}^{K_{4}} \int_{K_{2}}^{K_{3}} \int_{K_{1}}^{K_{2}}\left\{\left(c_{i m p}+c_{\min }\left\{\Lambda\left[A_{3}\left(K_{4}\right) \mid r\right]-\Lambda\left[A_{3}\left(u_{3}\right) \mid r\right]\right\}\right)\right. \\
& \times \lambda\left[A_{2}\left(u_{3}\right) \mid r\right] e^{-\left\{\Lambda\left[A_{2}\left(u_{3}\right) \mid r\right]-\Lambda\left[A_{2}\left(K_{3}\right) \mid r\right]\right\}} \\
& \times \lambda\left[A_{1}\left(u_{2}\right) \mid r\right] e^{-\left\{\Lambda\left[A_{1}\left(u_{2}\right) \mid r\right]-\Lambda\left[A_{1}\left(K_{2}\right) \mid r\right]\right\}} \\
& \left.\times f_{T_{K_{1} \mid r}}\left(u_{1}\right)\right\} d u_{1} d u_{2} d u_{3} \text {. }
\end{aligned}
$$

In general, for any of the intermediate subregions $\Omega_{l}, l=2,3, \ldots, n-1$, the number of possible previous imperfect repairs before that subregion can be $i=0,1, \ldots, l-2$. The first repair in $\Omega_{l}$ is imperfect and all subsequent repairs are minimal; given that at least one failure (hence, an imperfect repair) occurs in $\Omega_{l}$, when the number of possible previous imperfect repairs is $i=0$, the imperfect repair in $\Omega_{l}$ is the first in the warranty region; when $i=1$, the imperfect repair in $\Omega_{l}$ is the second in the warranty region, and so on.

Now, given the number of possible previous imperfect repairs $i$, where $i=0,1, \ldots, l-2$, and conditional on the time $u_{i+1}$ of the imperfect repair in $\Omega_{l}$, the corresponding conditional expected warranty servicing costs are 
given by

$$
\begin{cases}c_{i m p}+c_{\min }\left\{\Lambda\left[A_{1}\left(K_{l}\right) \mid r\right]-\Lambda\left[A_{1}\left(u_{1}\right) \mid r\right]\right\}, & i=0 \text { and } K_{l-1}<u_{1} \leq K_{l} \\ c_{i m p}+c_{\min }\left\{\Lambda\left[A_{2}\left(K_{l}\right) \mid r\right]-\Lambda\left[A_{2}\left(u_{2}\right) \mid r\right]\right\}, & i=1 \text { and } K_{l-1}<u_{2} \leq K_{l} \\ c_{i m p}+c_{\min }\left\{\Lambda\left[A_{3}\left(K_{l}\right) \mid r\right]-\Lambda\left[A_{3}\left(u_{3}\right) \mid r\right]\right\}, & i=2 \text { and } K_{l-1}<u_{3} \leq K_{l} \\ \vdots & \\ c_{i m p}+c_{\min }\left\{\Lambda\left[A_{l-1}\left(K_{l}\right) \mid r\right]-\Lambda\left[A_{l-1}\left(u_{l-1}\right) \mid r\right]\right\}, & i=l-2 \text { and } \\ 0, & K_{l-1}<u_{l-1} \leq K_{l}\end{cases}
$$

In the first case, the imperfect repair in $\Omega_{l}$ is the first. In the second case, the imperfect repair in $\Omega_{l}$ is the second, and hence, the first imperfect repair at time $u_{1}$ could have been in any of the subregions $\Omega_{2}, \Omega_{3}, \ldots, \Omega_{l-1}$. In the third case, the imperfect repair in $\Omega_{l}$ is the third, and hence, the first and second imperfect repairs at times $u_{1}$ and $u_{2}$ could have been in any two of the subregions $\Omega_{2}, \Omega_{3}, \ldots, \Omega_{l-1}$, and so on.

To derive the expected warranty servicing cost within the subregion $\Omega_{l}$, we remove the conditioning on the times to imperfect repair, $u_{1}, u_{2}, \ldots, u_{l}-$ 1 , to account for all possible combinations of subintervals in which the imperfect repairs have been performed. 
Therefore

$$
\begin{aligned}
& E\left[C_{r}^{\Omega_{l}}\left(\psi_{n}\right)\right]=\int_{K_{l-1}}^{K_{l}}\left\{\left(c_{i m p}+c_{\min }\left\{\Lambda\left[A_{1}\left(K_{l}\right) \mid r\right]-\Lambda\left[A_{1}\left(u_{1}\right) \mid r\right]\right\}\right)\right. \\
& \left.\times f_{T_{K_{1} \mid r}}\left(u_{1}\right)\right\} d u_{1} \\
& +\int_{K_{l-1}}^{K_{l}} \sum_{\left\{j_{1}\right\} \in J_{1, l-1}} \int_{K_{j_{1}-1}}^{K_{j_{1}}}\left\{\left(c_{i m p}+c_{\min }\left\{\Lambda\left[A_{2}\left(K_{l}\right) \mid r\right]-\Lambda\left[A_{2}\left(u_{2}\right) \mid r\right]\right\}\right)\right. \\
& \left.\times \lambda\left[A_{1}\left(u_{2}\right) \mid r\right] e^{-\left\{\Lambda\left[A_{1}\left(u_{2}\right) \mid r\right]-\Lambda\left[A_{1}\left(K_{j_{1}}\right) \mid r\right]\right\}} f_{T_{K_{1} \mid r}}\left(u_{1}\right)\right\} d u_{1} d u_{2} \\
& +\int_{K_{l-1}}^{K_{l}} \sum_{\forall\left\{j_{1}, j_{2}\right\} \in J_{2, l-1}} \int_{K_{j_{2}-1}}^{K_{j_{2}}} \int_{K_{j_{1}-1}}^{K_{j_{1}}}\left\{\left(c_{i m p}+c_{\min }\left\{\Lambda\left[A_{3}\left(K_{l}\right) \mid r\right]-\Lambda\left[A_{3}\left(u_{3}\right) \mid r\right]\right\}\right)\right. \\
& \times \lambda\left[A_{2}\left(u_{3}\right) \mid r\right] e^{-\left\{\Lambda\left[A_{2}\left(u_{3}\right) \mid r\right]-\Lambda\left[A_{2}\left(K_{j_{2}}\right) \mid r\right]\right\}} \\
& \times \lambda\left[A_{1}\left(u_{2}\right) \mid r\right] e^{-\left\{\Lambda\left[A_{1}\left(u_{2}\right) \mid r\right]-\Lambda\left[A_{1}\left(K_{j_{1}}\right) \mid r\right]\right\}} \\
& \left.\times f_{T_{K_{1} \mid r}}\left(u_{1}\right)\right\} d u_{1} d u_{2} d u_{3} \\
& +\ldots+ \\
& +\int_{K_{l-1}}^{K_{l}} \int_{K_{l-2}}^{K_{l-1}} \ldots \int_{K_{2}}^{K_{3}} \int_{K_{1}}^{K_{2}}\left\{\left(c_{i m p}+c_{\min }\left\{\Lambda\left[A_{l-1}\left(K_{l}\right) \mid r\right]-\Lambda\left[A_{l-1}\left(u_{l-1}\right) \mid r\right]\right\}\right)\right. \\
& \times \lambda\left[A_{l-2}\left(u_{l-1}\right) \mid r\right] e^{-\left\{\Lambda\left[A_{l-2}\left(u_{l-1}\right) \mid r\right]-\Lambda\left[A_{l-2}\left(K_{l-1}\right) \mid r\right]\right\}} \\
& \times \lambda\left[A_{l-3}\left(u_{l-2}\right) \mid r\right] e^{-\left\{\Lambda\left[A_{l-3}\left(u_{l-2}\right) \mid r\right]-\Lambda\left[A_{l-3}\left(K_{l-2}\right) \mid r\right]\right\}} \\
& \times \lambda\left[A_{1}\left(u_{2}\right) \mid r\right] e^{-\left\{\Lambda\left[A_{1}\left(u_{2}\right) \mid r\right]-\Lambda\left[A_{1}\left(K_{2}\right) \mid r\right]\right\}} \\
& \left.\times f_{T_{K_{1} \mid r}}\left(u_{1}\right)\right\} d u_{1} d u_{2} \ldots d u_{l-2} d u_{l-1}
\end{aligned}
$$

where the set $J_{i, l-1}$, defined on page 60 , provides all possible combinations of the subintervals in which the $i=1, \ldots, l-2$ possible previous imperfect repairs have been performed.

For the $n$-subregion warranty region, $\Omega_{n}$ denotes the last subregion within which all repairs are minimal. The conditional expected warranty servicing costs for $i=0,1, \ldots, n-1$ possible previous imperfect repairs is 
given by

$$
\begin{cases}c_{\min }\left\{\Lambda(K \mid r)-\Lambda\left(K_{n-1} \mid r\right)\right\}, & i=0 \\ c_{\min }\left\{\Lambda\left[A_{1}(K) \mid r\right]-\Lambda\left[A_{1}\left(K_{n-1}\right) \mid r\right]\right\}, & i=1 \\ c_{\min }\left\{\Lambda\left[A_{2}(K) \mid r\right]-\Lambda\left[A_{2}\left(K_{n-1}\right) \mid r\right]\right\}, & i=2 \\ \vdots & \\ c_{\min }\left\{\Lambda\left[A_{n-2}(K) \mid r\right]-\Lambda\left[A_{n-2}\left(K_{n-1}\right) \mid r\right]\right\}, & i=n-2\end{cases}
$$

if at least one failure has occurred in $\left[K_{n-1}, K\right)$, and zero if no failures have occurred in $\Omega_{n}$.

When unconditioning the costs in the last subregion $\Omega_{n}$, we must take into account the subregion in which the last imperfect repair occurred and the probability that no failures have occurred in the subregions between that subregion and $\Omega_{n}$. This probability is of the form

$$
e^{-\left\{\Lambda\left[A_{i}\left(K_{n-1}\right) \mid r\right]-\Lambda\left[A_{i}\left(K_{j_{i}}\right) \mid r\right]\right\}},
$$

where $i$ is the number of possible previous imperfect repairs and the time $u_{i}$ of the last (i.e. $i$-th) imperfect repair is in the interval $\left(K_{j_{i}-1}, K_{j_{i}}\right]$; this term implies that no failures (hence, imperfect repairs) have occurred in the interval $\left(K_{j_{i}}, K_{n-1}\right]$.

Finally, to derive the expected warranty servicing cost in the last subregion $\Omega_{n}$, we remove the conditioning using the summands of the density function derived in equation (4.10) and the above mentioned probabilities 
as follows:

$$
\begin{aligned}
& E\left[C_{r}^{\Omega_{n}}\left(\psi_{n}\right)\right]=c_{\min }\left\{\Lambda(K \mid r)-\Lambda\left(K_{n-1} \mid r\right)\right\} e^{-\left\{\Lambda\left(K_{n-1} \mid r\right)-\Lambda\left(K_{1} \mid r\right)\right\}} \\
& +\sum_{\forall\left\{j_{1}\right\} \in J_{1, n-1}} \int_{K_{j_{1}-1}}^{K_{j_{1}}}\left\{c_{\min }\left(\Lambda\left[A_{1}(K) \mid r\right]-\Lambda\left[A_{1}\left(K_{n-1}\right) \mid r\right]\right)\right. \\
& \left.\times e^{-\left\{\Lambda\left[A_{1}\left(K_{n-1}\right) \mid r\right]-\Lambda\left[A_{1}\left(K_{j_{1}}\right) \mid r\right]\right\}} f_{T_{K_{1} \mid r}}\left(u_{1}\right)\right\} d u_{1} \\
& +\sum_{\forall\left\{j_{1}, j_{2}\right\} \in J_{2, n-1}} \int_{K_{j_{2}-1}}^{K_{j_{2}}} \int_{K_{j_{1}-1}}^{K_{j_{1}}}\left\{c_{\min }\left(\Lambda\left[A_{2}(K) \mid r\right]-\Lambda\left[A_{2}\left(K_{n-1}\right) \mid r\right]\right)\right. \\
& \times e^{-\left\{\Lambda\left[A_{2}\left(K_{n-1}\right) \mid r\right]-\Lambda\left[A_{2}\left(K_{j_{2}}\right) \mid r\right]\right\}} \\
& \left.\times \lambda\left[A_{1}\left(u_{2}\right) \mid r\right] e^{-\left\{\Lambda\left[A_{1}\left(u_{2}\right) \mid r\right]-\Lambda\left[A_{1}\left(K_{j_{1}}\right) \mid r\right]\right\}} f_{T_{K_{1} \mid r}}\left(u_{1}\right)\right\} d u_{1} d u_{2} \\
& +\ldots+ \\
& +\int_{K_{n-2}}^{K_{n-1}} \cdots \int_{K_{2}}^{K_{3}} \int_{K_{1}}^{K_{2}}\left\{c_{\min }\left(\Lambda\left[A_{n-2}(K) \mid r\right]-\Lambda\left[A_{n-2}\left(K_{n-1}\right) \mid r\right]\right)\right. \\
& \times \lambda\left[A_{n-3}\left(u_{n-2}\right) \mid r\right] e^{-\left\{\Lambda\left[A_{n-3}\left(u_{n-2}\right) \mid r\right]-\Lambda\left[A_{n-3}\left(K_{n-2}\right) \mid r\right]\right\}} \\
& \times \quad: \\
& \times \lambda\left[A_{1}\left(u_{2}\right) \mid r\right] e^{-\left\{\Lambda\left[A_{1}\left(u_{2}\right) \mid r\right]-\Lambda\left[A_{1}\left(K_{2}\right) \mid r\right]\right\}} \\
& \left.\times \quad f_{T_{K_{1} \mid r}}\left(u_{1}\right)\right\} d u_{1} d u_{2} \ldots d u_{n-2}
\end{aligned}
$$

where the set $J_{i, n-1}$, defined on page 60 , provides all possible combinations of the subintervals in which the $i=1, \ldots, n-2$ previous imperfect repairs have been performed.

The expected total warranty servicing cost for sub-case A-(1), conditional on $R=r$, is given by

$$
E\left[C_{r}^{(1)}\left(\psi_{n}\right)\right]=E\left[C_{r}^{\Omega_{1}}\left(\psi_{n}\right)\right]+E\left[C_{r}^{\Omega_{2}}\left(\psi_{n}\right)\right]+\ldots+E\left[C_{r}^{\Omega_{n}}\left(\psi_{n}\right)\right]
$$

When summarizing the expected costs derived earlier $[(4.11),(4.12), \ldots$, (4.16)], notice that the virtual age corresponding to the number of possible previous imperfect repairs $i$, for $\Omega_{n}$ is different from that of $\Omega_{l}, l=$ $2, \ldots, n-1$. This is because in the last subregion $\Omega_{n}$ all repairs are minimal, while in the intermediate subregions $\Omega_{2}, \Omega_{3}, \ldots, \Omega_{n-1}$, the first repair 
is imperfect. Therefore, if $i$ is the number of possible previous imperfect repairs, when deriving the conditional costs in $\Omega_{l}, l=2,3, \ldots, n-1$, we have $i$ previous imperfect repairs and the $(i+1)$-th imperfect repair which is in $\Omega_{l}$. Hence, the expected total warranty servicing cost for sub-case A-(1) becomes

$$
\begin{aligned}
E\left[C_{r}^{(1)}\left(\psi_{n}\right)\right]= & c_{\min } \Lambda\left(K_{1} \mid r\right) \\
+ & c_{\min }\left[\Lambda(K \mid r)-\Lambda\left(K_{n-1} \mid r\right)\right] e^{-\left[\Lambda\left(K_{n-1} \mid r\right)-\Lambda\left(K_{1} \mid r\right)\right]} \\
+ & \sum_{l=2}^{n-1} \int_{K_{l-1}}^{K_{l}}\left\{\left(c_{i m p}+c_{\min }\left\{\Lambda\left[A_{1}\left(K_{l}\right) \mid r\right]-\Lambda\left[A_{1}\left(u_{1}\right) \mid r\right]\right\}\right.\right. \\
& \left.+c_{\min }\left\{\Lambda\left[A_{1}(K) \mid r\right]-\Lambda\left[A_{1}\left(K_{n-1}\right) \mid r\right]\right\} e^{-\left\{\Lambda\left[A_{1}\left(K_{n-1}\right) \mid r\right]-\Lambda\left[A_{1}\left(K_{l}\right) \mid r\right]\right\}}\right) \\
& \left.\times f_{T_{K_{1} \mid r}}\left(u_{1}\right)\right\} d u_{1} \\
+ & \sum_{l=3}^{n-1} \sum_{i=1}^{l-2} \int_{K_{l-1}}^{K_{l}}\left(\forall \{ j _ { 1 } , j _ { 2 } , \ldots , j _ { i } \} \in J _ { i , l - 1 } K _ { j _ { i } - 1 } \ldots \int _ { K _ { j _ { 2 } - 1 } K _ { j _ { 1 } - 1 } } ^ { K _ { j _ { i } } } \left\{\left[c_{i m p}+\right.\right.\right. \\
& +c_{\min }\left(\Lambda\left[A_{i+1}\left(K_{l}\right) \mid r\right]-\Lambda\left[A_{i+1}\left(u_{i+1}\right) \mid r\right]\right) \\
& \left.+c_{\min }\left(\Lambda\left[A_{i+1}(K) \mid r\right]-\Lambda\left[A_{i+1}\left(K_{n-1}\right) \mid r\right]\right) e^{-\left(\Lambda\left[A_{i+1}\left(K_{n-1}\right) \mid r\right]-\Lambda\left[A_{i+1}\left(K_{l}\right) \mid r\right]\right)}\right] \\
& \times \lambda\left[A_{i}\left(u_{i+1}\right) \mid r\right] e^{-\left\{\Lambda\left[A_{i}\left(u_{i+1}\right) \mid r\right]-\Lambda\left[A_{i}\left(K_{j_{i}}\right) \mid r\right]\right\}} \\
& \times \lambda\left[A_{i-1}\left(u_{i}\right) \mid r\right] e^{-\left\{\Lambda\left[A_{i-1}\left(u_{i}\right) \mid r\right]-\Lambda\left[A_{i-1}\left(K_{j_{i-1}}\right) \mid r\right]\right\}} \\
& \vdots \\
& \times \lambda\left[A_{1}\left(u_{2}\right) \mid r\right] e^{-\left\{\Lambda\left[A_{1}\left(u_{2}\right) \mid r\right]-\Lambda\left[A_{1}\left(K_{j_{1}}\right) \mid r\right]\right\}} \\
& \left.\left.\times f_{T_{K_{1} \mid r}}\left(u_{1}\right)\right\} d u_{1} d u_{2} \ldots d u_{i}\right) d u_{i+1}
\end{aligned}
$$

The expected cost in equation (4.17) is a function of the decision variables $K_{1}, K_{2}, \ldots, K_{n-1}$ and the warranty time limit $K$. At this point, we define a generic function $\zeta($.$) , such that$

$$
E\left[C_{r}^{(1)}\left(\psi_{n}\right)\right]=\zeta\left(K_{1}, K_{2}, \ldots, K_{n-1}, K\right) .
$$

The arguments of this function will later be modified to obtain the costs for sub-cases A-(2) and A-(3), and for Case B.

The expected cost derived by Chukova et al. [13], where instead of performing imperfect repair the failed product is replaced, is a special case 
of the cost in equation (4.17). When

$$
\delta_{1}=\delta_{2}=\ldots=\delta_{n-2}=1,
$$

equation (4.17) becomes

$$
\begin{aligned}
E\left[C_{r}^{(1)}\left(\psi_{n}\right)\right]= & c_{\min } \Lambda\left(K_{1} \mid r\right) \\
+ & c_{\min }\left[\Lambda(K \mid r)-\Lambda\left(K_{n-1} \mid r\right)\right] e^{-\left[\Lambda\left(K_{n-1} \mid r\right)-\Lambda\left(K_{1} \mid r\right)\right]} \\
+ & \sum_{l=2}^{n-1} \int_{K_{l-1}}^{K_{l}}\left\{c_{i m p}+c_{\min } \Lambda\left(K_{l}-u_{l-1} \mid r\right)+c_{\min }\left\{\Lambda\left(K-u_{l-1} \mid r\right)-\right.\right. \\
& \left.\quad-\Lambda\left(K_{n-1}-u_{l-1} \mid r\right)\right\} e^{-\left\{\Lambda\left(K_{n-1}-u_{l-1} \mid r\right)-\Lambda\left(K_{l}-u_{l-1} \mid r\right)\right\}} \\
& \left.\quad \times f_{T_{K_{l-1} \mid r}}\left(u_{l-1}\right)\right\} d u_{l-1}
\end{aligned}
$$

where $u_{l-1}$ is the time of the last perfect repair (replacement) which is in the subinterval $\left(K_{l-1}, K_{l}\right]$ (i.e. subregion $\left.\Omega_{l}\right)$, and $c_{i m p}=c_{p e r}$ is the cost of a perfect repair (replacement) [13].

The expected costs for sub-cases A-(2) and A-(3) are derived in a similar manner as the expected cost for sub-case A-(1). By adjusting the arguments of the function $\zeta($.$) in equation (4.18), we next define the expected$ costs $E\left[C_{r}^{(2)}\left(\psi_{n}\right)\right]$ and $E\left[C_{r}^{(3)}\left(\psi_{n}\right)\right]$ for Case A.

(2): $\mathrm{r}_{1} \leq \mathrm{r} \leq \mathrm{r}_{2}$

Figure 4.3 depicts the case where $r_{1} \leq r \leq r_{2}$. When $r_{1} \leq r \leq r_{2}$, the warranty over the subregions $\Omega_{1}, \Omega_{2}, \ldots, \Omega_{n-1}$ will expire due to exceeding the usage limits $L_{1}, L_{2}, \ldots, L_{n-1}$, at time points

$$
\tau_{1}=\frac{L_{1}}{r}, \quad \tau_{2}=\frac{L_{2}}{r}, \ldots, \quad \tau_{n-1}=\frac{L_{n-1}}{r},
$$

respectively. Therefore, the expected total warranty cost for sub-case A-(2), conditional on $R=r$, is similar to that of sub-case A-(1) in equation (4.17) except that $K_{i}$ are replaced by $\tau_{i}, i=1,2, \ldots, n-1$, respectively. Therefore, as in equation (4.18) we can write this cost in terms of the above variables, using the generic function $\zeta($.$) , as$

$$
E\left[C_{r}^{(2)}\left(\psi_{n}\right)\right]=\zeta\left(\tau_{1}, \tau_{2}, \ldots, \tau_{n-1}, K\right) .
$$




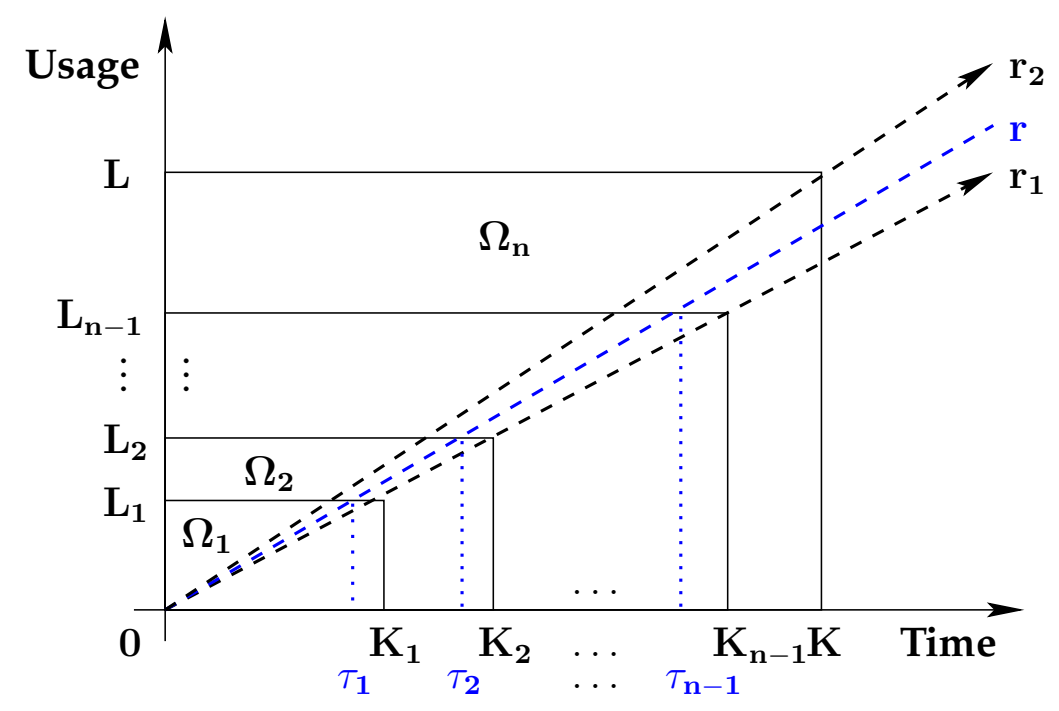

Figure 4.3: $r_{1} \leq r \leq r_{2}$.

(3): $\mathrm{r}_{1} \leq \mathrm{r}_{2} \leq \mathrm{r}$

Figure 4.4 depicts the case where $r_{1} \leq r_{2} \leq r$.

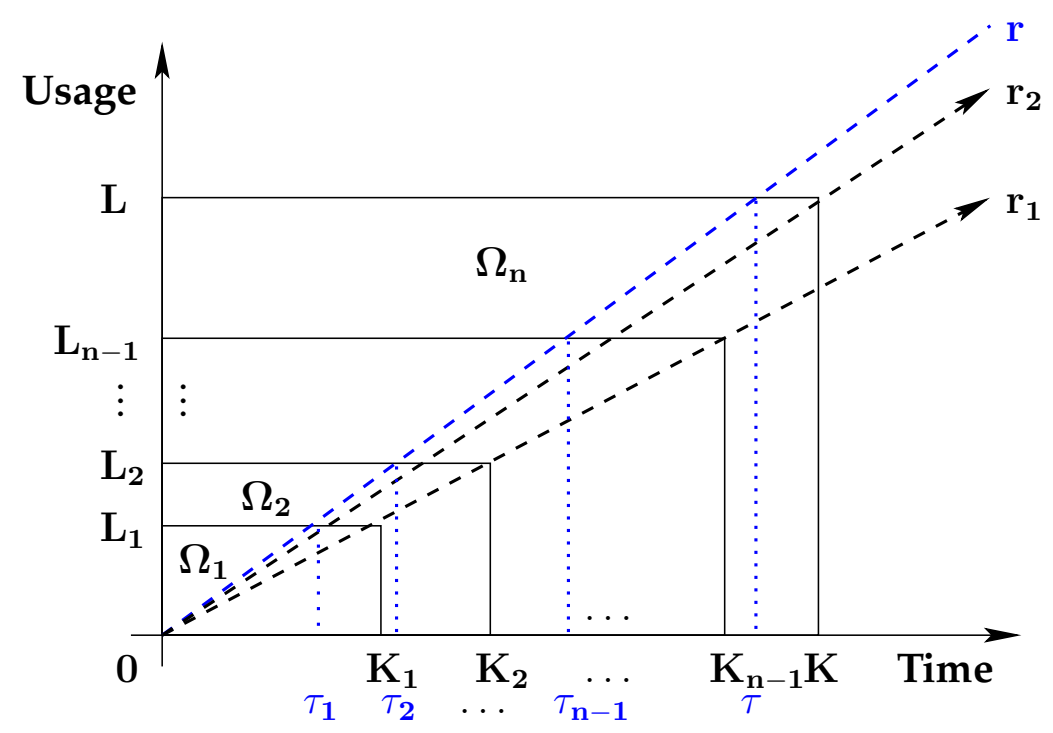

Figure 4.4: $r_{1} \leq r_{2} \leq r$. 
When $r_{1} \leq r_{2} \leq r$ the warranty over the entire region $\Omega$ expires at time

$$
\tau=\frac{L}{r}
$$

and the warranty over the subregions $\Omega_{1}, \Omega_{2}, \ldots, \Omega_{n-1}$ will expire at time points

$$
\tau_{1}=\frac{L_{1}}{r}, \quad \tau_{2}=\frac{L_{2}}{r}, \quad \ldots, \quad \tau_{n-1}=\frac{L_{n-1}}{r},
$$

respectively. The expected total warranty cost for sub-case A-(3), conditional on $R=r$, is similar to that of sub-case A-(2) in equation (4.19) except that $K$ is replaced by $\tau$. That is

$$
E\left[C_{r}^{(3)}(\psi)\right]=\zeta\left(\tau_{1}, \tau_{2}, \ldots, \tau_{n-1}, \tau\right)
$$

The expected total warranty cost for Case A, is derived by unconditioning the variable $R=r$. Therefore, for $r_{1} \leq r_{2}$, we have $E\left[C_{A}^{\Omega}\left(\psi_{n}\right)\right]=\int_{0}^{r_{1}} E\left[C_{r}^{(1)}\left(\psi_{n}\right)\right] d G(r)+\int_{r_{1}}^{r_{2}} E\left[C_{r}^{(2)}\left(\psi_{n}\right)\right] d G(r)+\int_{r_{2}}^{\infty} E\left[C_{r}^{(3)}\left(\psi_{n}\right)\right] d G(r)$

where $G(r)$ is the distribution of the usage rate $R$.

\subsubsection{Case B: $r_{2} \leq r_{1}$}

For Case $\mathrm{B}$, as for Case $\mathrm{A}$, we conditional on the usage rate $R=r$ and derive the expected warranty servicing costs for the following three subcases:

$\begin{array}{ll}\text { (1) } & r \leq r_{2} \leq r_{1} \\ \text { (2) } & r_{2} \leq r \leq r_{1} \\ \text { (3) } & r_{2} \leq r_{1} \leq r\end{array}$

Let $E\left[C_{r}^{(1)}\left(\psi_{n}\right)\right], E\left[C_{r}^{(2)}\left(\psi_{n}\right)\right]$, and $E\left[C_{r}^{(3)}\left(\psi_{n}\right)\right]$ now denote the expected warranty servicing costs, conditional on $R=r$, for the three sub-cases of Case $\mathrm{B}$ respectively [13]. Each of the expected costs $E\left[C_{r}^{(j)}\left(\psi_{n}\right)\right], j=1,2,3$, are 
the sum of the expected costs in each of the subregions $\Omega_{1}, \Omega_{2}, \ldots, \Omega_{n}$. That is

$$
E\left[C_{r}^{(j)}\left(\psi_{n}\right)=E\left[C_{r}^{\Omega_{1}}\left(\psi_{n}\right)\right]+E\left[C_{r}^{\Omega_{2}}\left(\psi_{n}\right)\right]+\ldots+E\left[C_{r}^{\Omega_{n}}\left(\psi_{n}\right)\right] .\right.
$$

We have not used the index $j$ in denoting the expected costs $E\left[C_{r}^{\Omega_{l}}\left(\psi_{n}\right)\right]$, $l=1,2, \ldots, n$, since it is clear by the context that these costs are specific to the corresponding sub-cases of Case B. The expected total warranty servicing cost for Case B is similar to that of Case A in equation (4.21), with the following adjustments.

(1): $r \leq r_{2} \leq r_{1}$

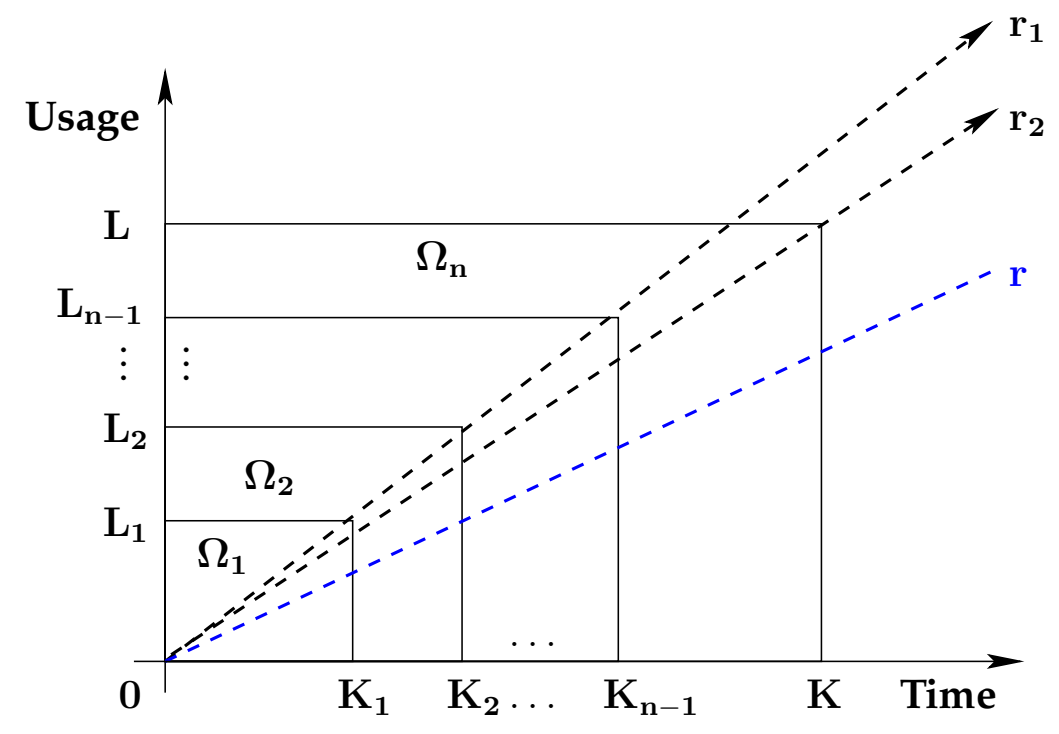

Figure 4.5: $r \leq r_{2} \leq r_{1}$

Figure 4.5 depicts the case where $r \leq r_{2} \leq r_{1}$. The expected total warranty servicing cost for sub-case B-(1) is the same as that of sub-case A-(1) in equation (4.17), and is given by

$$
E\left[C_{r}^{(1)}\left(\psi_{n}\right)\right]=\zeta\left(K_{1}, K_{2}, \ldots, K_{n-1}, K\right) .
$$


(2): $\mathrm{r}_{2} \leq \mathrm{r} \leq \mathrm{r}_{1}$

Figure 4.6 depicts the case where $r_{2} \leq r \leq r_{1}$.

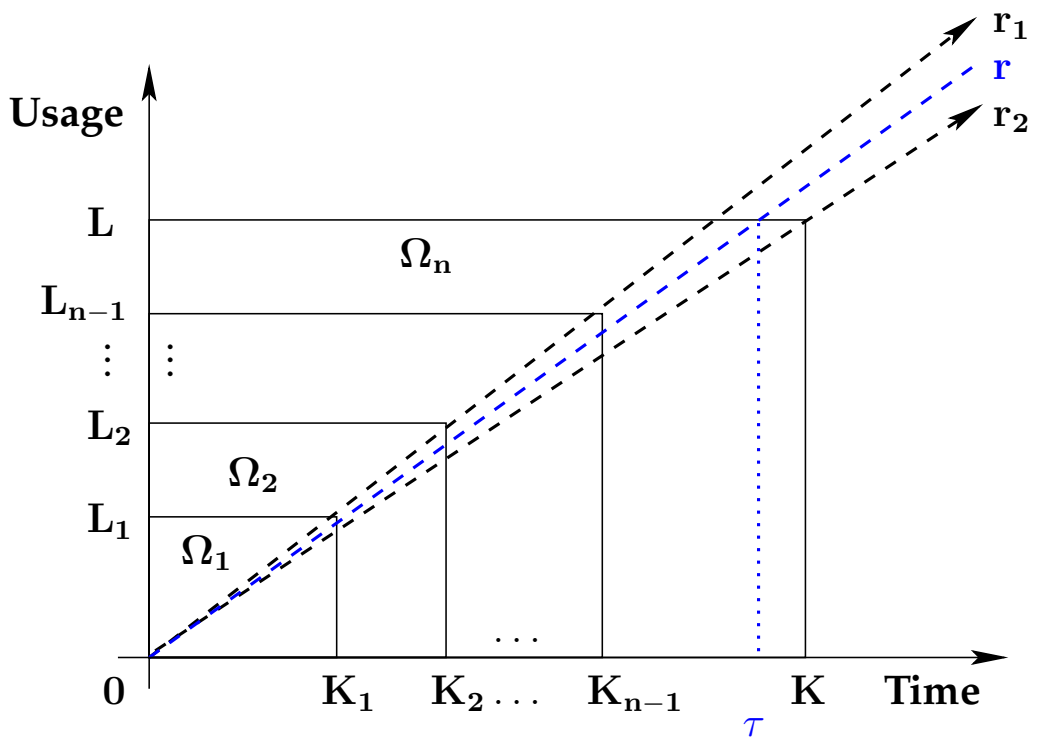

Figure 4.6: $r_{2} \leq r \leq r_{1}$

The expected total warranty servicing cost for sub-case B-(2) is similar to that of sub-case B-(1), with the exception that the warranty over the entire region $\Omega$ expires at time

$$
\tau=\frac{L}{r}
$$

and is given by

$$
E\left[C_{r}^{(2)}\left(\psi_{n}\right)\right]=\zeta\left(K_{1}, K_{2}, \ldots, K_{n-1}, \tau\right)
$$

(3): $\mathrm{r}_{2} \leq \mathrm{r}_{1} \leq \mathrm{r}$

Figure 4.7 depicts the case where $r_{2} \leq r_{1} \leq r$. 


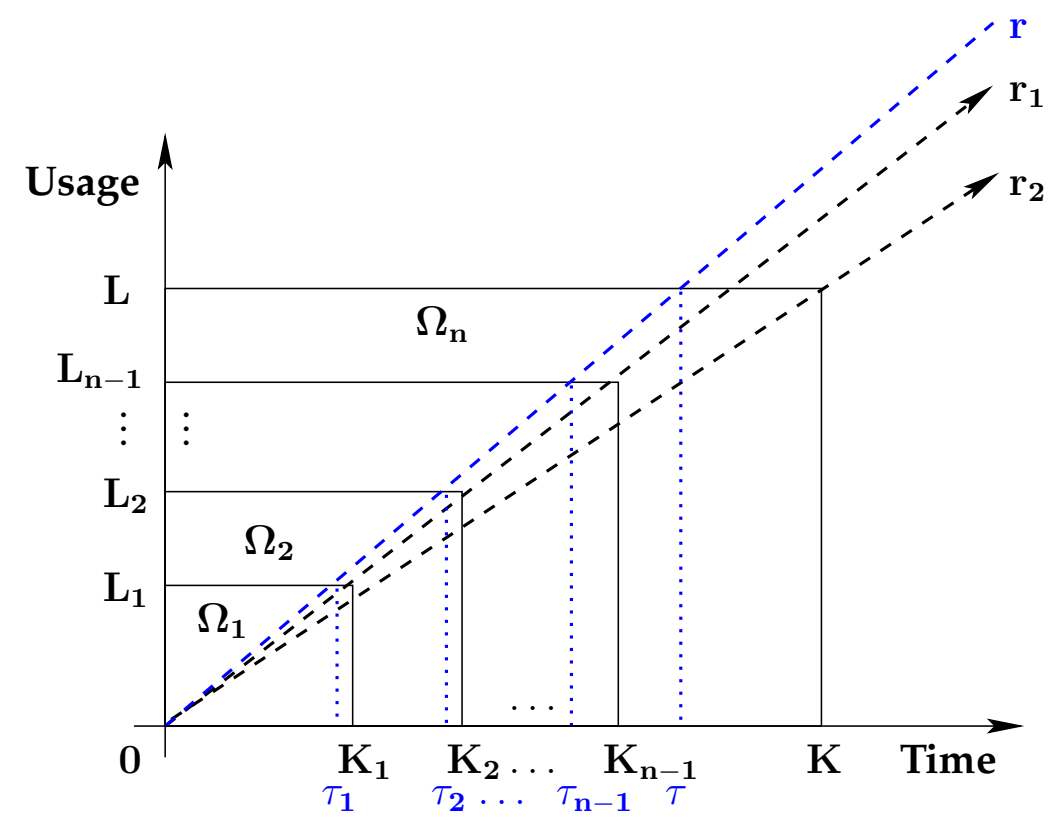

Figure 4.7: $r_{2} \leq r_{1} \leq r$

The expected total warranty servicing cost for sub-case B-(3) is similar to that of sub-case B-(2), with the exception that the warranty over the subregions $\Omega_{1}, \Omega_{2}, \ldots, \Omega_{n-1}$ expire at time points

$$
\tau_{1}=\frac{L_{1}}{r}, \quad \tau_{2}=\frac{L_{2}}{r}, \ldots, \quad \tau_{n-1}=\frac{L_{n-1}}{r},
$$

respectively. This cost is the same as the cost for sub-case A-(3). Therefore, the expected warranty servicing cost for sub-case B-(3) is given by

$$
E\left[C_{r}^{(3)}\left(\psi_{n}\right)\right]=\zeta\left(\tau_{1}, \tau_{2}, \ldots, \tau_{n-1}, \tau\right)
$$

Similar to the expected cost for Case A in equation (4.21), unconditioning the variable $R=r$, we get the expected total warranty cost for Case B. Therefore, for $r_{2} \leq r_{1}$, we have $E\left[C_{B}^{\Omega}\left(\psi_{n}\right)\right]=\int_{0}^{r_{2}} E\left[C_{r}^{(1)}\left(\psi_{n}\right)\right] d G(r)+\int_{r_{2}}^{r_{1}} E\left[C_{r}^{(2)}\left(\psi_{n}\right)\right] d G(r)+\int_{r_{1}}^{\infty} E\left[C_{r}^{(3)}\left(\psi_{n}\right)\right] d G(r)$. 
The results derived in this chapter will be illustrated numerically in Chapter 6, where the expected cost $E\left[C^{\Omega}\left(\psi_{n}\right)\right]$, given by equation (4.21) when $r_{1} \leq r_{2}$ and by equation (4.22) when $r_{2} \leq r_{1}$, is minimized to obtain the optimal decision variables

$$
\psi_{n}^{*}=\left(K_{1}^{*}, K_{2}^{*}, \ldots, K_{n-1}^{*}, r_{1}^{*}\right)
$$

that determine the partitions $\Omega_{1}, \Omega_{2}, \ldots, \Omega_{n}$. 


\section{Chapter 5}

\section{Intensity Reduction Model}

In this chapter, we discuss the second of the two imperfect repair models, namely the intensity reduction model, in detail and derive the distribution of the times to imperfect repair and the associated expected total warranty servicing cost. According to the intensity reduction model, the effect of an imperfect repair is characterized by the change in the conditional intensity function of the underlying failure process [16]. At any time, the conditional intensity function of the failure process after an imperfect repair is between the conditional intensity after a minimal repair and the conditional intensity after a perfect repair (replacement).

Chukova et al. [11] consider the failure rate functions of the first lifetime and second lifetime of a process and propose that the failure rate of the second lifetime distribution after an imperfect repair at time $u_{1}$ with degree $0<\delta<1$ is

$$
\lambda_{1}(t)=\lambda(t)-\delta\left[\lambda(t)-\lambda\left(t-u_{1}\right)\right]
$$

for $t>u_{1}$, where $\lambda(t)$ is the failure rate of the first lifetime distribution. Chukova et al. [12] extend this to a generalized form, such that, after the $i$-th imperfect repair, the failure rate of the lifetime distribution becomes

$$
\lambda_{i}(t)=\lambda_{i-1}(t)-\delta\left[\lambda_{i-1}(t)-\lambda_{i-1}\left(t-u_{i}\right)\right],
$$


for $t>u_{i}$, where $u_{i}$ is the time of the $i$-th imperfect repair. This model suggests that the imperfect repair can only undo the damage that the product has accumulated since the last imperfect repair. The intensity reduction model considered in this research is a modification of this model. Here an imperfect repair can undo the damage that the product has accumulated since it was first put into use. This model is more appropriate for modeling failures of a single-component product. These models are analogous to the virtual age models proposed by Kijima [29]. We now proceed to explain in detail the intensity reduction model used in this research.

\subsection{Model Formulation}

Let $\{\tilde{N}(t \mid r) ; t \geq 0\}$ denote the one-dimensional counting process conditional on $R=r$, and let $\tilde{\lambda}(t \mid r)$ be the intensity function of the process. If the times to imperfect repair are non-random, then the conditional intensity function of the process is the intensity function of the process. Therefore, conditional on the times to imperfect repair being $u_{1}, u_{2}, \ldots, u_{n-2}$, the intensity function of the process changes as follows. Let the initial intensity function of the failure process, conditional on $R=r$, be

$$
\lambda_{0}(t \mid r)=\lambda(t \mid r),
$$

where $\lambda(t \mid r)$ is an increasing function of time, and is equal to the failure rate function of the original product. As before, let $\delta_{i}$ denote the degree of the $i$-th imperfect repair, where $i=1,2, \ldots, n-2$. Conditional on $R=r$, the intensity function after the first imperfect repair at time $u_{1}$ with degree $\delta_{1}$, becomes

$$
\lambda_{1}(t \mid r)=\lambda(t \mid r)-\delta_{1}\left[\lambda(t \mid r)-\lambda\left(t-u_{1} \mid r\right)\right],
$$

where $u_{1}<t \leq u_{2}$. Therefore, after a minimal repair, i.e. when $\delta_{1}=0$, we have

$$
\lambda_{1}(t \mid r)=\lambda(t \mid r)
$$


and after a replacement at time $u_{1}$, i.e. when $\delta_{1}=1$, we have

$$
\lambda_{1}(t \mid r)=\lambda\left(t-u_{1} \mid r\right)
$$

which is the initial intensity function of the process at time $t-u_{1}$. The intensity function after the second imperfect repair at time $u_{2}$ with degree $\delta_{2}$ is

$$
\lambda_{2}(t \mid r)=\lambda_{1}(t \mid r)-\delta_{2}\left[\lambda_{1}(t \mid r)-\lambda\left(t-u_{2} \mid r\right)\right],
$$

where $u_{2}<t \leq u_{3}$. Therefore, when $\delta_{2}=0$, we have

$$
\lambda_{2}(t \mid r)=\lambda_{1}(t \mid r)
$$

and when $\delta_{2}=1$, we have

$$
\lambda_{2}(t \mid r)=\lambda\left(t-u_{2} \mid r\right)
$$

which is the initial intensity of the process at time $t-u_{2}$. In general, after the $i$-th imperfect repair at time $u_{i}$ with degree $\delta_{i}$, for $u_{i}<t \leq u_{i+1}$, the intensity function becomes

$$
\lambda_{i}(t \mid r)=\lambda_{i-1}(t \mid r)-\delta_{i}\left[\lambda_{i-1}(t \mid r)-\lambda\left(t-u_{i} \mid r\right)\right],
$$

which, when $\delta_{i}=0$ (minimal repair), reduces to

$$
\lambda_{i}(t \mid r)=\lambda_{i-1}(t \mid r)
$$

and when $\delta_{i}=1$ (replacement), reduces to

$$
\lambda_{i}(t \mid r)=\lambda\left(t-u_{i} \mid r\right),
$$

which is the initial intensity of the process at time $t-u_{i}$. Therefore, conditional on $R=r$, the intensity function of the process $\tilde{N}(t \mid r)$, given the 
times to imperfect repair $u_{1}, u_{2}, \ldots, u_{n-2}$, is given by

$$
\tilde{\lambda}(t \mid r)= \begin{cases}\lambda(t \mid r), & 0 \leq t \leq u_{1} \\ \lambda_{1}(t \mid r), & u_{1} \leq t \leq u_{2} \\ \lambda_{2}(t \mid r), & u_{2} \leq t \leq u_{3} \\ \vdots & \\ \lambda_{i}(t \mid r), & u_{i} \leq t \leq u_{i+1} \\ \vdots & \\ \lambda_{n-2}(t \mid r), & u_{n-2} \leq t<\infty\end{cases}
$$

See Figure 5.1 for an example of this intensity function.

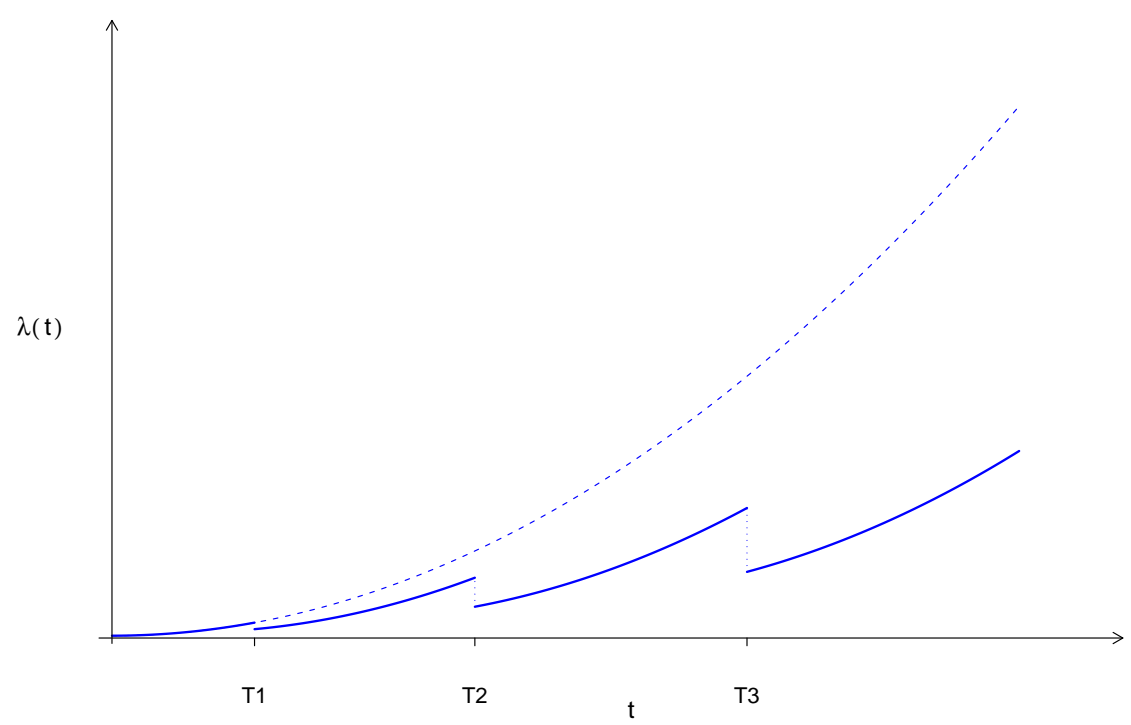

Figure 5.1: Intensity function following imperfect repairs of degree 0.5.

The cumulative intensity function of the process is given by

$$
\tilde{\Lambda}(t \mid r)=\int_{0}^{t} \tilde{\lambda}(s \mid r) d s .
$$

Therefore, for $i>0$ and $u_{i}<t \leq u_{i+1}$, the expected number of failures in 
the subinterval $\left(u_{i}, t\right]$ is given by

$$
\begin{aligned}
E\left[\tilde{N}(t \mid r)-\tilde{N}\left(u_{i} \mid r\right)\right] & =\int_{u_{i}}^{t} \tilde{\lambda}(s \mid r) d s \\
& =\int_{u_{i}}^{t} \lambda_{i}(s \mid r) d s \\
& =\tilde{\Lambda}(t \mid r)-\tilde{\Lambda}\left(u_{i} \mid r\right),
\end{aligned}
$$

where $\lambda_{i}(t \mid r)$ is the intensity function after the $i$-th imperfect repair and is a function of all previous times to imperfect repair. For simplification and clarity purposes, let $\tilde{\Lambda}(t \mid r)-\tilde{\Lambda}\left(u_{i} \mid r\right)$ be denoted by $\Lambda_{i}(t \mid r)$. That is

$$
\Lambda_{i}(t \mid r)=\tilde{\Lambda}(t \mid r)-\tilde{\Lambda}\left(u_{i} \mid r\right),
$$

for $i=1,2, \ldots, n-2$. For $t<u_{1}$, i.e. before the first imperfect repair, we have

$$
\tilde{\Lambda}(t \mid r)=\Lambda(t \mid r)=\int_{0}^{t} \lambda(s \mid r) d s
$$

which is the expected number of minimal repairs (failures) before the first imperfect repair. These expected numbers are conditional on the times to imperfect repair being $u_{1}, u_{2}, \ldots, u_{n-2}$.

In the following section, we derive the density functions of the times to imperfect repair for the intensity reduction model. These density functions are derived in exactly the same manner as those derived for the age reduction model. Also, these density functions have a similar structure as those derived in the previous chapter, with one difference: in the density functions for the age reduction model, the change in the intensity function after each repair is only in the virtual age and hence, the function itself does not change; in the density functions for the intensity reduction model, the change is in the intensity function itself. Hence, where we had

$$
\lambda\left[A_{i}(t) \mid r\right],
$$


we will now have

$$
\lambda_{i}(t \mid r)
$$

and where we had

$$
\Lambda\left[A_{i}(t) \mid r\right]-\Lambda\left[A_{i}\left(K_{j}\right) \mid r\right]
$$

we will now have

$$
\Lambda_{i}(t \mid r)-\Lambda_{i}\left(K_{j} \mid r\right)
$$

Therefore, we will omit the derivation process, and only provide the final results.

\subsection{Times to Imperfect Repair}

As before, $T_{1 \mid r}$ denotes the time of the first failure conditional on $R=r$, and $T_{K_{l} \mid r}, l=1,2, \ldots, n-2$ denotes the time of the first failure after $K_{l}$, i.e. in subregion $\Omega_{l+1}$. See Chapter 4 for the distribution of the first failure time $T_{1 \mid r}[14,21]$. The distribution function and density function of the first failure after $K_{1}, T_{K_{1} \mid r}$, are given by

$$
F_{T_{K_{1} \mid r}}(t)=P\left\{T_{K_{1} \mid r} \leq t\right\}=1-e^{-\left[\Lambda(t \mid r)-\Lambda\left(K_{1} \mid r\right)\right]},
$$

and

$$
f_{T_{K_{1} \mid r}}(t)=\lambda(t \mid r) e^{-\left[\Lambda(t \mid r)-\Lambda\left(K_{1} \mid r\right)\right]}
$$

respectively, where $\lambda(t \mid r)$ is the initial intensity function, conditional on $R=r$. For $t>K_{2}$, the distribution function and density function of the time to first failure after $K_{2}, T_{K_{2} \mid r}$, are given by

$$
\begin{aligned}
F_{T_{K_{2} \mid r}}(t)= & P\left\{T_{K_{2} \mid r} \leq t\right\} \\
= & \int_{K_{1}}^{K_{2}}\left(1-e^{-\left\{\Lambda_{1}(t \mid r)-\Lambda_{1}\left(K_{2} \mid r\right)\right\}}\right) f_{T_{K_{1} \mid r}}\left(u_{1}\right) d u_{1}+e^{-\left[\Lambda\left(K_{2} \mid r\right)-\Lambda\left(K_{1} \mid r\right)\right]} \\
& -e^{-\left[\Lambda(t \mid r)-\Lambda\left(K_{1} \mid r\right)\right]}
\end{aligned}
$$


and

$$
\begin{aligned}
f_{T_{K_{2} \mid r}}(t) & =\frac{d}{d t} F_{T_{K_{2} \mid r}}(t) \\
& =\int_{K_{1}}^{K_{2}} \lambda_{1}(t \mid r) e^{-\left\{\Lambda_{1}(t \mid r)-\Lambda_{1}\left(K_{2} \mid r\right)\right\}} f_{T_{K_{1} \mid r}}\left(u_{1}\right) d u_{1}+f_{T_{K_{1} \mid r}}(t),
\end{aligned}
$$

respectively, where $\lambda_{1}(t \mid r)$ is the intensity function after the first imperfect repair at time $u_{1}$, and $f_{T_{K_{1} \mid r}}(t)$ is defined in equation (5.4). As derived in the previous chapter, for $t>K_{3}$, the distribution function of $T_{K_{3} \mid r}$ is given by

$$
\begin{aligned}
F_{T_{K_{3} \mid r}}(t)= & P\left\{T_{K_{3} \mid r} \leq t\right\} \\
= & \int_{K_{2}}^{K_{3}} \int_{K_{1}}^{K_{2}}\left\{\left(1-e^{-\left\{\Lambda_{2}(t \mid r)-\Lambda_{2}\left(K_{3} \mid r\right)\right\}}\right) \lambda_{1}\left(u_{2} \mid r\right) e^{-\left\{\Lambda_{1}\left(u_{2} \mid r\right)-\Lambda_{1}\left(K_{2} \mid r\right)\right\}}\right. \\
& \left.f_{T_{K_{1} \mid r}}\left(u_{1}\right)\right\} d u_{1} d u_{2} \\
& +\int_{K_{2}}^{K_{3}}\left(1-e^{-\left\{\Lambda_{1}(t \mid r)-\Lambda_{1}\left(K_{3} \mid r\right)\right\}}\right) e^{-\left\{\Lambda_{1}\left(K_{3} \mid r\right)-\Lambda_{1}\left(K_{3} \mid r\right)\right\}} f_{T_{K_{1} \mid r}}\left(u_{1}\right) d u_{1} \\
& +\int_{K_{1}}^{K_{2}}\left(1-e^{-\left\{\Lambda_{1}(t \mid r)-\Lambda_{1}\left(K_{3} \mid r\right)\right\}}\right) e^{-\left\{\Lambda_{1}\left(K_{3} \mid r\right)-\Lambda_{1}\left(K_{2} \mid r\right)\right\}} f_{T_{K_{1} \mid r}}\left(u_{1}\right) d u_{1} \\
& +\left(1-e^{\left\{\Lambda(t \mid r)-\Lambda\left(K_{3} \mid r\right)\right\}}\right) e^{-\left\{\Lambda\left(K_{3} \mid r\right)-\Lambda\left(K_{1} \mid r\right)\right\}} .
\end{aligned}
$$

The functions $\Lambda_{1}(. \mid r)$ and $\Lambda_{2}(. \mid r)$ are the cumulative intensity functions after the first imperfect repair and second imperfect repair, respectively. The double integral is over the subintervals $\left(K_{1}, K_{2}\right]$ and $\left(K_{2}, K_{3}\right]$, where an imperfect repair could have been performed. In the third summand, the exponent term implies that no failures have occurred in the subinterval $\left(K_{2}, t\right]$. In other words, there have been no imperfect repairs in the 
previous subinterval $\left(K_{2}, K_{3}\right]$. The density function of $T_{K_{3} \mid r}$ is given by

$$
\begin{aligned}
f_{T_{K_{3} \mid r}}(t)= & \frac{d}{d t} F_{T_{K_{3} \mid r}}(t) \\
= & \int_{K_{2}}^{K_{3}} \int_{K_{1}}^{K_{2}}\left\{\lambda_{2}(t \mid r) e^{-\left\{\Lambda_{2}(t \mid r)-\Lambda_{2}\left(K_{3} \mid r\right)\right\}}\right. \\
& \left.\lambda_{1}\left(u_{2} \mid r\right) e^{-\left\{\Lambda_{1}\left(u_{2} \mid r\right)-\Lambda_{1}\left(K_{2} \mid r\right)\right\}} f_{T_{K_{1} \mid r}}\left(u_{1}\right)\right\} d u_{1} d u_{2} \\
& +\int_{K_{2}}^{K_{3}} \lambda_{1}(t \mid r) e^{-\left\{\Lambda_{1}(t \mid r)-\Lambda_{1}\left(K_{3} \mid r\right)\right\}} f_{T_{K_{1} \mid r}}\left(u_{1}\right) d u_{1} \\
& +\int_{K_{1}}^{K_{2}} \lambda_{1}(t \mid r) e^{-\left\{\Lambda_{1}(t \mid r)-\Lambda_{1}\left(K_{2} \mid r\right)\right\}} f_{T_{K_{1} \mid r}}\left(u_{1}\right) d u_{1} \\
& +f_{T_{K_{1} \mid r}}(t) .
\end{aligned}
$$

Similarly, in deriving the density function of the time of the first failure after $K_{4}$, namely $T_{K_{4} \mid r}$, we must consider the cases where none, one, two or three imperfect repairs have been performed before $K_{4}$. The density function for $T_{K_{4} \mid r}$ is given on the following page.

Note that the first summand is the case where there has been at least one failure (and therefore an imperfect repair) in each of the subintervals, $\left(K_{1}, K_{2}\right],\left(K_{2}, K_{3}\right]$, and $\left(K_{3}, K_{4}\right]$, before $K_{4}$. The last summand is the case where no failures have occurred in the subintervals $\left(K_{1}, K_{2}\right],\left(K_{2}, K_{3}\right]$, and $\left(K_{3}, K_{4}\right]$, i.e, no failures in the subinterval $\left(K_{1}, K_{4}\right]$. The three summands with double integrals each represents the case where there has been at least one failure in each of the two corresponding subintervals and the next three summands with single integrals each represents the case where there has been at least one failure in the corresponding subinterval. 
The density function for $T_{K_{4} \mid r}$ is

$$
\begin{aligned}
& f_{T_{K_{4} \mid r}}(t)=\int_{K_{3}}^{K_{4}} \int_{K_{2}}^{K_{3}} \int_{K_{1}}^{K_{2}}\left\{\lambda_{3}(t \mid r) e^{-\left\{\Lambda_{3}(t \mid r)-\Lambda_{3}\left(K_{4} \mid r\right)\right\}}\right. \\
& \times \lambda_{2}\left(u_{3} \mid r\right) e^{-\left\{\Lambda_{2}\left(u_{3} \mid r\right)-\Lambda_{2}\left(K_{3} \mid r\right)\right\}} \\
& \left.\times \lambda_{1}\left(u_{2} \mid r\right) e^{-\left\{\Lambda_{1}\left(u_{2} \mid r\right)-\Lambda_{1}\left(K_{2} \mid r\right)\right\}} f_{T_{K_{1} \mid r}}\left(u_{1}\right)\right\} d u_{1} d u_{2} d u_{3} \\
& +\int_{K_{2}}^{K_{3}} \int_{K_{1}}^{K_{2}}\left\{\lambda_{2}(t \mid r) e^{-\left\{\Lambda_{2}(t \mid r)-\Lambda_{2}\left(K_{3} \mid r\right)\right\}}\right. \\
& \left.\times \lambda_{1}\left(u_{2} \mid r\right) e^{-\left\{\Lambda_{1}\left(u_{2} \mid r\right)-\Lambda_{1}\left(K_{2} \mid r\right)\right\}} f_{T_{K_{1} \mid r}}\left(u_{1}\right)\right\} d u_{1} d u_{2} \\
& +\int_{K_{3}}^{K_{4}} \int_{K_{2}}^{K_{3}}\left\{\lambda_{2}(t \mid r) e^{-\left\{\Lambda_{2}(t \mid r)-\Lambda_{2}\left(K_{4} \mid r\right)\right\}}\right. \\
& \left.\times \lambda_{1}\left(u_{2} \mid r\right) e^{-\left\{\Lambda_{1}\left(u_{2} \mid r\right)-\Lambda_{1}\left(K_{3} \mid r\right)\right\}} f_{T_{K_{1} \mid r}}\left(u_{1}\right)\right\} d u_{1} d u_{2} \\
& +\int_{K_{3}}^{K_{4}} \int_{K_{1}}^{K_{2}}\left\{\lambda_{2}(t \mid r) e^{-\left\{\Lambda_{2}(t \mid r)-\Lambda_{2}\left(K_{4} \mid r\right)\right\}}\right. \\
& \left.\times \lambda_{1}\left(u_{2} \mid r\right) e^{-\left\{\Lambda_{1}\left(u_{2} \mid r\right)-\Lambda_{1}\left(K_{2} \mid r\right)\right\}} f_{T_{K_{1} \mid r}}\left(u_{1}\right)\right\} d u_{1} d u_{2} \\
& +\int_{K_{3}}^{K_{4}} \lambda_{1}(t \mid r) e^{-\left\{\Lambda_{1}(t \mid r)-\Lambda_{1}\left(K_{4} \mid r\right)\right\}} f_{T_{K_{1} \mid r}}\left(u_{1}\right) d u_{1} \\
& +\int_{K_{2}}^{K_{3}} \lambda_{1}(t \mid r) e^{-\left\{\Lambda_{1}(t \mid r)-\Lambda_{1}\left(K_{3} \mid r\right)\right\}} f_{T_{K_{1} \mid r}}\left(u_{1}\right) d u_{1} \\
& +\int_{K_{1}}^{K_{2}} \lambda_{1}(t \mid r) e^{-\left\{\Lambda_{1}(t \mid r)-\Lambda_{1}\left(K_{2} \mid r\right)\right\}} f_{T_{K_{1} \mid r}}\left(u_{1}\right) d u_{1} \\
& +f_{T_{K_{1} \mid r}}(t) \text {. }
\end{aligned}
$$

where the functions $\Lambda_{1}(. \mid r), \Lambda_{2}(. \mid r)$ and $\Lambda_{3}(. \mid r)$ are the cumulative 
intensity functions after the first imperfect repair, the second imperfect repair and the third imperfect repair, respectively.

In general, to derive the density function of the first repair after $K_{l}$, namely $T_{K_{l \mid r}}$, where $l=1, \ldots, n-2$ for the $n$-subregion strategy, we need to consider all possible combinations of imperfect repairs in the previous subregions $\Omega_{2}, \Omega_{3}, \ldots, \Omega_{l}$. As before, let $i$ denote the possible number of previous imperfect repairs. For $T_{K_{l \mid r}}$, the time of the first failure after $K_{l}$, we have $i=0,1, \ldots, l-1$ possible previous imperfect repairs. By conditioning on the times to imperfect repair $u_{1}, u_{2}, \ldots, u_{l-1}$ and then removing the conditioning, we account for all possible times to imperfect repair within the corresponding subintervals. Then the density function of the time to first failure after $K_{l}, T_{K_{l} \mid r}$, is given by

$$
\begin{aligned}
f_{T_{K_{l} \mid r}}(t)= & f_{T_{K_{1} \mid r}}(t) \\
+ & \sum_{i=1}^{l-1} \sum_{\forall\left\{j_{1}, j_{2} \ldots, j_{i}\right\} \in J_{i, l} K_{K_{j_{i}-1}}} \int_{K_{j_{2}-1}}^{K_{j_{i}}} \int_{K_{j_{1}-1}}^{K_{j_{2}}}\left\{\lambda_{i}(t \mid r) e^{\left.-\left\{\Lambda_{i}(t \mid r)-\Lambda_{i}\left(K_{j_{i}} \mid r\right)\right]\right\}}\right. \\
& \times \lambda_{i-1}\left(u_{i} \mid r\right) e^{\left.-\left\{\Lambda_{i-1}\left(u_{i} \mid r\right)-\Lambda_{i-1}\left(K_{j_{i-1}} \mid r\right)\right]\right\}} \\
& \vdots \\
& \times \lambda_{1}\left(u_{2} \mid r\right) e^{\left.-\left\{\Lambda_{1}\left(u_{2} \mid r\right)-\Lambda_{1}\left(K_{j_{1}} \mid r\right)\right]\right\}} \\
& \left.\times f_{T_{K_{1} \mid r}}\left(u_{1}\right)\right\} d u_{1} d u_{2} \ldots d u_{i}
\end{aligned}
$$

where the functions $\lambda_{i}(. \mid r)$ and $\Lambda_{i}(. \mid r)$ are defined in equation (5.1) and equation (5.3) respectively. For $i>0$, the set

$$
J_{i, l}=\left\{\left\{j_{1}, \ldots, j_{i}\right\}:\left\{j_{1}, \ldots, j_{i}\right\} \subseteq\{2, \ldots, l\} \text { and } j_{1}<\cdots<j_{i}\right\}
$$

provides all possible combinations of $\left\{K_{j_{1}}, K_{j_{2}} \ldots, K_{j_{i}}\right\}$ which generate these subintervals (see page 60 for details on $J_{i, l}$ ).

Having derived the density function, we proceed to deriving the expected warranty servicing cost over the warranty region for the intensity 
reduction model. This cost is derived in the same manner as the cost for the age reduction model in Chapter 4. Like the density functions, the structure of this cost is the same as that of the cost derived for the age reduction model. The differences between the derived cost for the two models are mentioned earlier on page 87 and in addition, the expected number of failures in $\left(u_{i}, t\right]$ for the age reduction model is denoted by

$$
\Lambda\left[A_{i}(t) \mid r\right]-\Lambda\left[A_{i}\left(u_{i}\right) \mid r\right]
$$

and for the intensity reduction model is denoted by

$$
\Lambda_{i}(t \mid r) \text {. }
$$

See page 86 for the definition of the cumulative intensity function $\Lambda_{i}(t \mid r)$. Therefore, in the following section, we will derive the expected warranty servicing costs in a more concise manner.

\subsection{Warranty Servicing Costs}

Like before, let $E\left[C^{\Omega}\left(\psi_{n}\right)\right]$ denote the total expected warranty servicing cost over the warranty region $\Omega$. Since the repair strategy is restricted, in determining the total expected warranty cost $E\left[C^{\Omega}\left(\psi_{n}\right)\right]$, we consider the two cases; $r_{1} \leq r_{2}$ and $r_{2} \leq r_{1}$, where $r_{1}>0$ and $r_{2}>0$. As before, $E\left[C_{A}^{\Omega}\left(\psi_{n}\right)\right]$ denotes the total expected warranty servicing cost for the first case $r_{1} \leq r_{2}$ and $E\left[C_{B}^{\Omega}\left(\psi_{n}\right)\right]$ denotes the total expected warranty servicing cost for the second case $r_{2} \leq r_{1}$. That is,

- Case A: $E\left[C^{\Omega}\left(\psi_{n}\right)\right]=E\left[C_{A}^{\Omega}\left(\psi_{n}\right)\right]$

- Case B: $E\left[C^{\Omega}\left(\psi_{n}\right)\right]=E\left[C_{B}^{\Omega}\left(\psi_{n}\right)\right]$

In the following sections, we derive the expected warranty costs for Case A and Case B. 


\subsubsection{Case A: $r_{1} \leq r_{2}$}

As in the age reduction model, we subdivide the warranty region based on the rates $r_{1}$ and $r_{2}$ and conditional on the usage rate $R=r$ derive the expected warranty servicing costs for the following three sub-cases;

$\begin{array}{ll}\text { (1) } & r \leq r_{1} \leq r_{2} \\ \text { (2) } & r_{1} \leq r \leq r_{2} \\ \text { (3) } & r_{1} \leq r_{2} \leq r\end{array}$

The expected warranty servicing costs, conditional on $R=r$, for the three sub-cases are denoted by $E\left[C_{r}^{(1)}\left(\psi_{n}\right)\right], E\left[C_{r}^{(2)}\left(\psi_{n}\right)\right]$, and $E\left[C_{r}^{(3)}\left(\psi_{n}\right)\right]$ respectively. Then the expected total warranty cost for Case $A$ is given by

$E\left[C_{A}^{\Omega}\left(\psi_{n}\right)\right]=\int_{0}^{r_{1}} E\left[C_{r}^{(1)}\left(\psi_{n}\right)\right] d G(r)+\int_{r_{1}}^{r_{2}} E\left[C_{r}^{(2)}\left(\psi_{n}\right)\right] d G(r)+\int_{r_{2}}^{\infty} E\left[C_{r}^{(3)}\left(\psi_{n}\right)\right] d G(r)$.

Each of the expected costs $E\left[C_{r}^{(j)}\left(\psi_{n}\right)\right], j=1,2,3$, are the sum of the corresponding expected costs in each of the subregions $\Omega_{1}, \Omega_{2}, \ldots, \Omega_{n}$, which are denoted by

$$
E\left[C_{r}^{\Omega_{1}}\left(\psi_{n}\right)\right], E\left[C_{r}^{\Omega_{2}}\left(\psi_{n}\right)\right], \ldots, E\left[C_{r}^{\Omega_{n}}\left(\psi_{n}\right)\right] .
$$

Since these costs are clear in context, the use of the index $j$ is omitted. Therefore, the expected warranty servicing cost conditional on $R=r$, for the three sub-cases, $j=1,2,3$, is given by

$$
E\left[C_{r}^{(j)}\left(\psi_{n}\right)=E\left[C_{r}^{\Omega_{1}}\left(\psi_{n}\right)\right]+E\left[C_{r}^{\Omega_{2}}\left(\psi_{n}\right)\right]+\ldots+E\left[C_{r}^{\Omega_{3}}\left(\psi_{n}\right)\right] .\right.
$$

The main results of this chapter are the density function derived in equation (5.5), and the expected warranty servicing costs for Case A, $E\left[C_{A}^{\Omega}\left(\psi_{n}\right)\right]$, and Case B, $E\left[C_{B}^{\Omega}\left(\psi_{n}\right)\right]$, for the intensity reduction model which we derive in the following sections. As in the age reduction model, we will only derive the expected cost for sub-case A-(1), since the expected costs for A(2) and A-(3) and Case B can be obtained in a similar manner with minor adjustments. 
(1) $\mathrm{r} \leq \mathrm{r}_{1} \leq \mathrm{r}_{2}$

Let $c_{\text {min }}$ and $c_{i m p}$ denote the cost of a minimal repair and the cost of an imperfect repair, respectively. The costs $c_{\min }$ and $c_{i m p}$ are assumed to be constant. Later, in the numerical example, we will adjust the cost $c_{i m p}$ based on the degrees of the imperfect repairs.

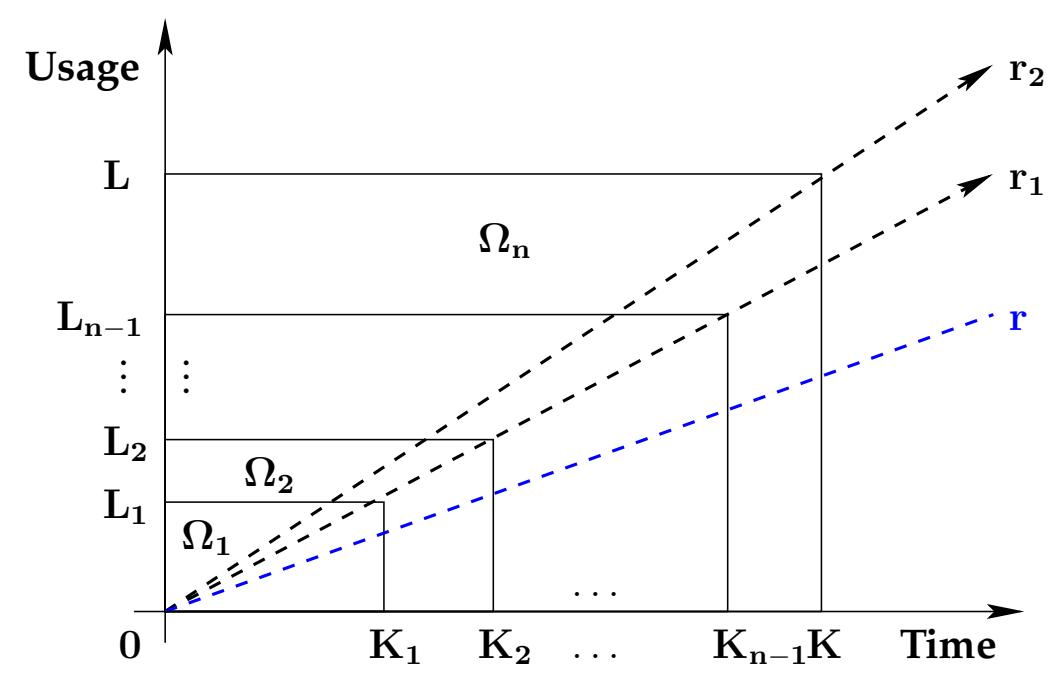

Figure 5.2: $\mathbf{r} \leq \mathbf{r}_{1} \leq \mathbf{r}_{2}$.

The expected warranty servicing cost in the first subregion $\Omega_{1}$ is not conditional on times to imperfect repair, since all repairs in this subregion are minimal, and therefore, the warranty servicing cost in this subregion is given by

$$
\begin{cases}c_{\min } \tilde{N}\left(K_{1} \mid r\right), & \tilde{N}\left(K_{1} \mid r\right)>0 \\ 0, & \text { otherwise },\end{cases}
$$

where $\tilde{N}\left(K_{1} \mid r\right)$ is the number of failures in the subinterval $\left[0, K_{1}\right]$ and the expected warranty servicing cost in $\Omega_{1}$ is given by

$$
\begin{cases}c_{\min } \Lambda\left(K_{1} \mid r\right), & \tilde{N}\left(K_{1} \mid r\right)>0 \\ 0, & \text { otherwise }\end{cases}
$$


where

$$
\Lambda\left(K_{1} \mid r\right)=\int_{0}^{K_{1}} \lambda(s \mid r) d s
$$

That is

$$
E\left[C_{r}^{\Omega_{1}}\left(\psi_{n}\right)\right]=c_{\min } \Lambda\left(K_{1} \mid r\right) .
$$

The first failure in the second subregion $\Omega_{2}$ is followed by an imperfect repair and all consequent failures are followed by minimal repairs. Conditional on the time of the imperfect repair being $u_{1}$, the conditional warranty servicing cost in subregion $\Omega_{2}$ is given by

$$
\begin{cases}c_{i m p}+c_{\min }\left[\tilde{N}\left(K_{2} \mid r\right)-\tilde{N}\left(u_{1} \mid r\right)\right], & u_{1} \leq K_{2} \\ 0, & u_{1}>K_{2}\end{cases}
$$

where $\tilde{N}\left(K_{2} \mid r\right)-\tilde{N}\left(u_{1} \mid r\right)$ denotes the number of failures (minimal repairs) in the subinterval $\left(u_{1}, K_{2}\right]$. The expected conditional warranty servicing cost in $\Omega_{2}$ is given by

$$
\begin{cases}c_{i m p}+c_{\min } \Lambda_{1}\left(K_{2} \mid r\right), & u_{1} \leq K_{2} \\ 0, & u_{1}>K_{2}\end{cases}
$$

where

$$
\Lambda_{1}\left(K_{2} \mid r\right)=\int_{u_{1}}^{K_{2}} \lambda_{1}(s \mid r) d s
$$

is the cumulative intensity function after the first imperfect repair at time $u_{1}$ and the expected number of minimal repairs in the subinterval $\left(u_{1}, K_{2}\right]$. Removing the conditioning on $u_{1}$, we get

$$
E\left[C_{r}^{\Omega_{2}}\left(\psi_{n}\right)\right]=\int_{K_{1}}^{K_{2}}\left[c_{i m p}+c_{\min } \Lambda_{1}\left(K_{2} \mid r\right)\right] f_{T_{K_{1} \mid r}}\left(u_{1}\right) d u_{1}
$$

The first failure in the third subregion $\Omega_{3}$ is also followed by an imperfect repair and all subsequent repairs in this subregion are followed by minimal repairs. Therefore, conditional on the time of the first imperfect repair 
$u_{1}$ and the time of the second imperfect repair $u_{2}$, the conditional warranty servicing cost in subregion $\Omega_{3}$ is given by

$$
\begin{cases}c_{i m p}+c_{\min }\left[\tilde{N}\left(K_{3} \mid r\right)-\tilde{N}\left(u_{1} \mid r\right)\right], & K_{2}<u_{1} \leq K_{3} \\ c_{i m p}+c_{\min }\left[\tilde{N}\left(K_{3} \mid r\right)-\tilde{N}\left(u_{2} \mid r\right)\right], & u_{1} \leq K_{2} \text { and } u_{2} \leq K_{3} \\ 0, & \text { otherwise }\end{cases}
$$

where $\tilde{N}\left(K_{3} \mid r\right)-\tilde{N}\left(u_{1} \mid r\right)$ and $\tilde{N}\left(K_{3} \mid r\right)-\tilde{N}\left(u_{2} \mid r\right)$ count the number of minimal repairs in the subintervals $\left(u_{1}, K_{3}\right]$ and $\left(u_{2}, K_{3}\right]$ respectively. The expected conditional warranty servicing cost in subregion $\Omega_{3}$ is

$$
\begin{cases}c_{i m p}+c_{\min } \Lambda_{1}\left(K_{3} \mid r\right), & K_{2}<u_{1} \leq K_{3} \\ c_{i m p}+c_{\min } \Lambda_{2}\left(K_{3} \mid r\right), & u_{1} \leq K_{2} \text { and } u_{2} \leq K_{3} \\ 0, & \text { otherwise }\end{cases}
$$

where $\Lambda_{1}\left(K_{3} \mid r\right)$ and $\Lambda_{2}\left(K_{3} \mid r\right)$ are the expected number of minimal repairs in the subintervals $\left(u_{1}, K_{3}\right]$ and $\left(u_{2}, K_{3}\right]$ respectively. On removing the conditioning on $u_{1}$ and $u_{2}$, we get the expected warranty servicing cost in subregion $\Omega_{3}$, i.e.

$$
\begin{aligned}
E\left[C_{r}^{\Omega_{3}}\left(\psi_{n}\right)\right]= & \int_{K_{2}}^{K_{3}}\left[c_{i m p}+c_{\min } \Lambda_{1}\left(K_{3} \mid r\right)\right] f_{T_{K_{1} \mid r}}\left(u_{1}\right) d u_{1} \\
+ & \int_{K_{2}}^{K_{3}} \int_{K_{1}}^{K_{2}}\left\{\left[c_{i m p}+c_{\min } \Lambda_{2}\left(K_{3} \mid r\right)\right] \lambda_{1}\left(u_{2} \mid r\right) e^{-\left\{\Lambda_{1}\left(u_{2} \mid r\right)-\Lambda_{1}\left(K_{2} \mid r\right)\right\}}\right. \\
& \left.\quad \times f_{T_{K_{1} \mid r}}\left(u_{1}\right)\right\} d u_{1} d u_{2}
\end{aligned}
$$

Similar to the cost in $\Omega_{3}$, the conditional warranty servicing cost in subregion $\Omega_{4}$, conditional on the times to imperfect repair $u_{1}, u_{2}$ and $u_{3}$, is given by

$$
\begin{cases}c_{i m p}+c_{\min }\left[\tilde{N}\left(K_{4} \mid r\right)-\tilde{N}\left(u_{1} \mid r\right)\right], & K_{3}<u_{1} \leq K_{4} \\ c_{i m p}+c_{\min }\left[\tilde{N}\left(K_{4} \mid r\right)-\tilde{N}\left(u_{2} \mid r\right)\right], & u_{1} \leq K_{2} \text { and } K_{3}<u_{2} \leq K_{4} \\ c_{i m p}+c_{\min }\left[\tilde{N}\left(K_{4} \mid r\right)-\tilde{N}\left(u_{2} \mid r\right)\right], & K_{2}<u_{1} \leq K_{3} \text { and } K_{3}<u_{2} \leq K_{4} \\ c_{i m p}+c_{\min }\left[\tilde{N}\left(K_{4} \mid r\right)-\tilde{N}\left(u_{3} \mid r\right)\right], & u_{1} \leq K_{2}, u_{2} \leq K_{3} \text { and } u_{3} \leq K_{4} \\ 0, & \text { otherwise }\end{cases}
$$


and the expected conditional warranty servicing cost in this subregion is given by

$$
\begin{cases}c_{i m p}+c_{\text {min }} \Lambda_{1}\left(K_{4} \mid r\right), & K_{3}<u_{1} \leq K_{4} \\ c_{i m p}+c_{\text {min }} \Lambda_{2}\left(K_{4} \mid r\right), & u_{1} \leq K_{2} \text { and } K_{3}<u_{2} \leq K_{4} \\ c_{i m p}+c_{\text {min }} \Lambda_{2}\left(K_{4} \mid r\right), & K_{2}<u_{1} \leq K_{3} \text { and } K_{3}<u_{2} \leq K_{4} \\ c_{i m p}+c_{\text {min }} \Lambda_{3}\left(K_{4} \mid r\right), & u_{1} \leq K_{2}, u_{2} \leq K_{3} \text { and } u_{3} \leq K_{4} \\ 0, & \text { otherwise }\end{cases}
$$

where $\Lambda_{1}\left(K_{4} \mid r\right), \Lambda_{2}\left(K_{4} \mid r\right)$ and $\Lambda_{3}\left(K_{4} \mid r\right)$ are the expected number of minimal repairs in the subintervals $\left(u_{1}, K_{4}\right],\left(u_{2}, K_{4}\right]$ and $\left(u_{3}, K_{4}\right]$ respectively. On removing the conditioning on $u_{1}, u_{2}$ and $u_{3}$, we get the expected warranty servicing cost in subregion $\Omega_{4}$, i.e.

$$
\begin{aligned}
E\left[C_{r}^{\Omega_{4}}\left(\psi_{n}\right)\right]= & \int_{K_{3}}^{K_{4}}\left[c_{i m p}+c_{\text {min }} \Lambda_{1}\left(K_{4} \mid r\right)\right] f_{T_{K_{1} \mid r}}\left(u_{1}\right) d u_{1} \\
+ & \int_{K_{3}}^{K_{4}} \int_{K_{1}}^{K_{2}}\left\{\left[c_{i m p}+c_{\min } \Lambda_{2}\left(K_{4} \mid r\right)\right]\right. \\
& \left.\times \lambda_{1}\left(u_{2} \mid r\right) e^{-\left\{\Lambda_{1}\left(u_{2} \mid r\right)-\Lambda_{1}\left(K_{2} \mid r\right)\right\}} f_{T_{K_{1} \mid r}}\left(u_{1}\right)\right\} d u_{1} d u_{2} \\
+ & \int_{K_{3}}^{K_{4}} \int_{K_{2}}^{K_{3}}\left\{\left[c_{i m p}+c_{\min } \Lambda_{2}\left(K_{4} \mid r\right)\right]\right. \\
& \left.\times \lambda_{1}\left(u_{2} \mid r\right) e^{-\left\{\Lambda_{1}\left(u_{2} \mid r\right)-\Lambda_{1}\left(K_{3} \mid r\right)\right\}} f_{T_{K_{1} \mid r}}\left(u_{1}\right)\right\} d u_{1} d u_{2} \\
+ & \int_{K_{3}}^{K_{4}} \int_{K_{2}}^{K_{3}} \int K_{K_{1}}\left\{\left[c_{i m p}+c_{m i n} \Lambda_{3}\left(K_{4} \mid r\right)\right]\right. \\
& \quad \times \lambda_{2}\left(u_{3} \mid r\right) e^{-\left\{\Lambda_{2}\left(u_{3} \mid r\right)-\Lambda_{2}\left(K_{3} \mid r\right)\right\}} \lambda_{1}\left(u_{2} \mid r\right) e^{-\left\{\Lambda_{1}\left(u_{2} \mid r\right)-\Lambda_{1}\left(K_{2} \mid r\right)\right\}} \\
& \left.\times f_{T_{K_{1} \mid r}}\left(u_{1}\right)\right\} d u_{1} d u_{2} d u_{3} .
\end{aligned}
$$

In any of the intermediate subregions $\Omega_{l}, l=2,3, \ldots, n-1$, the possible number of previous imperfect repairs before the subregion, can be $i=0,1, \ldots, l-2$, since all repairs in the first subregion are minimal. As derived in the age reduction model, conditional on the times to imperfect repair being $u_{1}, u_{2}, \ldots, u_{l-1}$, the conditional expected warranty cost in 
subregion $\Omega_{l}$ corresponding to the number of possible imperfect repairs $i=0,1, \ldots, l-2$ before the subregion and the $(i+1)$-th imperfect repair which is in $\Omega_{l}$, is given by

$$
\left\{\begin{array}{cc}
c_{i m p}+c_{\text {min }} \Lambda_{1}\left(K_{l} \mid r\right), & i=0 \text { and } K_{l-1}<u_{1} \leq K_{l} \\
c_{i m p}+c_{\text {min }} \Lambda_{2}\left(K_{l} \mid r\right), & i=1 \text { and } K_{l-1}<u_{2} \leq K_{l} \\
c_{i m p}+c_{m i n} \Lambda_{3}\left(K_{l} \mid r\right), & i=2 \text { and } K_{l-1}<u_{3} \leq K_{l} \\
\vdots & \vdots \\
c_{i m p}+c_{m i n} \Lambda_{l-1}\left(K_{l} \mid r\right), & i=l-2 \text { and } K_{l-1}<u_{l-1} \leq K_{l} \\
0, & \text { otherwise } .
\end{array}\right.
$$

To derive the expected warranty servicing cost within the subregion $\Omega_{l}$, we remove the conditioning on the times to imperfect repair to get

$$
\begin{aligned}
& E\left[C_{r}^{\Omega_{l}}\left(\psi_{n}\right)\right]=\int_{K_{l-1}}^{K_{l}}\left[c_{i m p}+c_{\min } \Lambda_{1}\left(K_{l} \mid r\right)\right] f_{T_{K_{1} \mid r}}\left(u_{1}\right) d u_{1} \\
& +\int_{K_{l-1}}^{K_{l}}\left(\sum _ { \forall \{ j _ { 1 } \} \in J _ { 1 , l - 1 } } \int _ { K _ { j _ { 1 } - 1 } } ^ { K _ { j _ { 1 } } } \left\{\left[c_{i m p}+c_{\min } \Lambda_{2}\left(K_{l} \mid r\right)\right]\right.\right. \\
& \left.\left.\times \lambda_{1}\left(u_{2} \mid r\right) e^{-\left\{\Lambda_{1}\left(u_{2} \mid r\right)-\Lambda_{1}\left(K_{j_{1}} \mid r\right)\right\}} f_{T_{K_{1} \mid r}}\left(u_{1}\right)\right\} d u_{1}\right) d u_{2} \\
& +\int_{K_{l-1}}^{K_{l}}\left(\sum _ { \forall \{ j _ { 1 } , j _ { 2 } \} \in J _ { 2 , l - 1 } } \int _ { K _ { j _ { 2 } - 1 } } ^ { K _ { j _ { 2 } } } \int _ { K _ { j _ { 1 } - 1 } } ^ { K _ { j _ { 1 } } } \left\{\left[c_{i m p}+c_{\min } \Lambda_{3}\left(K_{l} \mid r\right)\right]\right.\right. \\
& \times \lambda_{2}\left(u_{3} \mid r\right) e^{-\left\{\Lambda_{2}\left(u_{3} \mid r\right)-\Lambda_{2}\left(K_{j_{2}} \mid r\right)\right\}} \\
& \left.\left.\times \lambda_{1}\left(u_{2} \mid r\right) e^{-\left\{\Lambda_{1}\left(u_{2} \mid r\right)-\Lambda_{1}\left(K_{j_{1}} \mid r\right)\right\}} f_{T_{K_{1} \mid r}}\left(u_{1}\right)\right\} d u_{1} d u_{2}\right) d u_{3} \\
& +\ldots+ \\
& +\int_{K_{l-1}}^{K_{l}} \int_{K_{l-2}}^{K_{l-1}} \cdots \int_{K_{2}}^{K_{3}} \int_{K_{1}}^{K_{2}}\left\{\left[c_{i m p}+c_{\min } \Lambda_{l-1}\left(K_{l} \mid r\right)\right]\right. \\
& \times \lambda_{l-2}\left(u_{l-1} \mid r\right) e^{-\left\{\Lambda_{l-2}\left(u_{l-1} \mid r\right)-\Lambda_{l-2}\left(K_{l-1} \mid r\right)\right\}} \\
& \times \lambda_{l-3}\left(u_{l-2} \mid r\right) e^{-\left\{\Lambda_{l-3}\left(u_{l-2} \mid r\right)-\Lambda_{l-3}\left(K_{l-2} \mid r\right)\right\}} \\
& \vdots \\
& \times \lambda_{1}\left(u_{2} \mid r\right) e^{\left.-\left\{\Lambda_{1}\left(u_{2} \mid r\right)-\Lambda_{1}\left(K_{2} \mid r\right)\right]\right\}} \\
& \left.\times f_{T_{K_{1} \mid r}}\left(u_{1}\right)\right\} d u_{1} d u_{2} \ldots d u_{l-2} d u_{l-1}
\end{aligned}
$$


where the set $J_{i, l-1}, i=1, \ldots, l-2$ (see page 60 ), provides all combinations of the subintervals in which the $i$ possible previous imperfect repairs have been performed. The conditional expected warranty servicing costs in the last subregion $\Omega_{n}$, for $i$ previous imperfect repairs before $\Omega_{n}$ is

$$
\begin{cases}c_{\min }\left[\Lambda(K \mid r)-\Lambda\left(K_{n-1} \mid r\right)\right], & i=0 \\ c_{\min }\left[\Lambda_{1}(K \mid r)-\Lambda_{1}\left(K_{n-1} \mid r\right)\right], & i=1 \\ c_{\min }\left[\Lambda_{2}(K \mid r)-\Lambda_{2}\left(K_{n-1} \mid r\right)\right], & i=2 \\ \vdots & \\ c_{\text {min }}\left[\Lambda_{n-2}(K \mid r)-\Lambda_{n-2}\left(K_{n-1} \mid r\right)\right], & i=n-2\end{cases}
$$

if at least one failure has occurred in $\Omega_{n}$ and zero otherwise. To derive the expected warranty servicing cost in the last subregion $\Omega_{n}$, we remove the conditioning on the times to imperfect repair as follows

$$
\begin{aligned}
& E\left[C_{r}^{\Omega_{n}}\left(\psi_{n}\right)\right]=c_{\min }\left[\Lambda(K \mid r)-\Lambda\left(K_{n-1} \mid r\right)\right] e^{-\left\{\Lambda\left(K_{n-1} \mid r\right)-\Lambda\left(K_{1} \mid r\right)\right\}} \\
& +\sum_{\forall\left\{j_{1}\right\} \in J_{1, n-1}} \int_{K_{j_{1}-1}}^{K_{j_{1}}}\left\{c_{\min }\left[\Lambda_{1}(K \mid r)-\Lambda_{1}\left(K_{n-1} \mid r\right)\right]\right. \\
& \left.\times e^{-\left\{\Lambda_{1}\left(K_{n-1} \mid r\right)-\Lambda_{1}\left(K_{j_{1}} \mid r\right)\right\}} f_{T_{K_{1} \mid r}}\left(u_{1}\right)\right\} d u_{1} \\
& +\sum_{\forall\left\{j_{1}, j_{2}\right\} \in J_{2, n-1}} \int_{K_{j_{2}-1}}^{K_{j_{2}}} \int_{K_{j_{1}-1}}^{K_{j_{1}}}\left\{c_{\min }\left\{\Lambda_{2}(K \mid r)-\Lambda_{2}\left(K_{n-1} \mid r\right)\right\}\right. \\
& \times e^{-\left\{\Lambda_{2}\left(K_{n-1} \mid r\right)-\Lambda_{2}\left(K_{j_{2}} \mid r\right)\right\}} \lambda_{1}\left(u_{2} \mid r\right) e^{-\left\{\Lambda_{1}\left(u_{2} \mid r\right)-\Lambda_{1}\left(K_{j_{1}} \mid r\right)\right\}} \\
& \left.\times f_{T_{K_{1} \mid r}}\left(u_{1}\right)\right\} d u_{1} d u_{2} \\
& +\ldots+ \\
& +\int_{K_{n-2}}^{K_{n-1}} \int_{K_{n-3}}^{K_{n-2}} \cdots \int_{K_{2}}^{K_{3}} \int_{K_{1}}^{K_{2}}\left\{c_{\min }\left\{\Lambda_{n-2}(K \mid r)-\Lambda_{n-2}\left(K_{n-1} \mid r\right)\right\}\right. \\
& \times \lambda_{n-3}\left(u_{n-2} \mid r\right) e^{-\left\{\Lambda_{n-3}\left(u_{n-2} \mid r\right)-\Lambda_{n-3}\left(K_{n-2} \mid r\right)\right\}} \\
& \times \lambda_{n-4}\left(u_{n-3} \mid r\right) e^{-\left\{\Lambda_{n-4}\left(u_{n-3} \mid r\right)-\Lambda_{n-4}\left(K_{n-3} \mid r\right)\right\}} \\
& \vdots \\
& \times \lambda_{1}\left(u_{2} \mid r\right) e^{\left.-\left\{\Lambda_{1}\left(u_{2} \mid r\right)-\Lambda_{1}\left(K_{2} \mid r\right)\right]\right\}} \\
& \left.\times f_{T_{K_{1} \mid r}}\left(u_{1}\right)\right\} d u_{1} d u_{2} \ldots d u_{n-3} d u_{n-2} \text {, }
\end{aligned}
$$


where the set $J_{i, n-1}, i=1, \ldots, n-2$ provides all possible combinations of the subintervals in which the $i$ previous imperfect repairs have been performed (see page 60 for details on the set $J_{i, n-1}$ ). For $i=1,2, \ldots, n-2$, the probability

$$
e^{-\left\{\Lambda_{i}\left(K_{n-1} \mid r\right)-\Lambda_{i}\left(K_{j_{i}} \mid r\right)\right\}},
$$

reflects the event that the last imperfect repair was performed in the subinterval $\left(K_{j_{i}-1}, K_{j_{i}}\right.$ ), i.e. no failures have occurred in the subregions between $\Omega_{j_{i}}$ and $\Omega_{n}$. Having derived the costs in the $n$ subregions, we now derive the expected cost for sub-case A-(1). Conditional on $R=r$, this cost is given by

$$
E\left[C_{r}^{(1)}\left(\psi_{n}\right)\right]=E\left[C_{r}^{\Omega_{1}}\left(\psi_{n}\right)\right]+E\left[C_{r}^{\Omega_{2}}\left(\psi_{n}\right)\right]+\ldots+E\left[C_{r}^{\Omega_{n}}\left(\psi_{n}\right)\right] .
$$

On summarizing the equations $[(5.6),(5.7), \ldots,(5.11)]$ derived for each subregion, as we did for the age reduction model, the expected warranty servicing cost for sub-case A-(1) becomes

$$
\begin{aligned}
E\left[C_{r}^{(1)}\left(\psi_{n}\right)\right]= & c_{\min } \Lambda\left(K_{1} \mid r\right) \\
+ & c_{\min }\left[\Lambda(K \mid r)-\Lambda\left(K_{n-1} \mid r\right)\right] e^{-\left\{\Lambda\left(K_{n-1} \mid r\right)-\Lambda\left(K_{1} \mid r\right)\right\}} \\
+ & \sum_{l=2}^{n-2} \int_{K_{l-1}}^{K_{l}}\left\{\left[c_{i m p}+c_{\min } \Lambda_{1}\left(K_{l} \mid r\right)\right]+c_{\min }\left\{\Lambda_{1}(K \mid r)-\Lambda_{1}\left(K_{n-1} \mid r\right)\right\}\right] \\
& \left.\quad \times e^{-\left\{\Lambda_{1}\left(K_{n-1} \mid r\right)-\Lambda_{1}\left(K_{l} \mid r\right)\right\}} f_{T_{K_{1} \mid r}}\left(u_{1}\right)\right\} d u_{1} \\
+ & \sum_{l=3}^{n-1} \sum_{i=1}^{l-2} \int_{K_{l-1}}^{K_{l}}\left(\sum _ { \forall \{ j _ { 1 } , j _ { 2 } , \ldots , j _ { i } \} \in J _ { i , l - 1 } } \int _ { K _ { j _ { i } - 1 } } ^ { K _ { j _ { i } } } \ldots \int _ { K _ { j _ { 2 } - 1 } } ^ { K _ { j _ { 2 } } } \int _ { K _ { j _ { 1 } - 1 } } ^ { K _ { j _ { 1 } } } \left\{\left[c_{i m p}\right.\right.\right. \\
& +c_{\min } \Lambda_{i+1}\left(K_{l} \mid r\right)+c_{\min }\left\{\Lambda_{i+1}(K \mid r)-\Lambda_{i+1}\left(K_{n-1} \mid r\right)\right\} \\
& \left.e^{-\left(\Lambda_{i+1}\left(K_{n-1} \mid r\right)-\Lambda_{i+1}\left(K_{l} \mid r\right)\right)}\right] \\
& \times \lambda_{i}\left(u_{i+1} \mid r\right) e^{-\left\{\Lambda_{i}\left(u_{i+1} \mid r\right)-\Lambda_{i}\left(K_{j_{i}} \mid r\right)\right\}} \\
& \times \lambda_{i-1}\left(u_{i} \mid r\right) e^{-\left\{\Lambda_{i-1}\left(u_{i} \mid r\right)-\Lambda_{i-1}\left(K_{j_{i-1}} \mid r\right)\right\}} \\
& \vdots \\
& \times \lambda_{1}\left(u_{2} \mid r\right) e^{-\left\{\Lambda_{1}\left(u_{2} \mid r\right)-\Lambda_{1}\left(K_{j_{1}} \mid r\right)\right\}} \\
& \left.\left.\times f_{T_{K_{1} \mid r}}\left(u_{1}\right)\right\} d u_{1} d u_{2} \ldots d u_{i}\right) d u_{i+1}
\end{aligned}
$$


The expected cost in equation (5.12) is a function of the decision variables $K_{1}, K_{2}, \ldots, K_{n-1}$ and the warranty time limit $K$. At this point, we define a generic function $\xi($.$) , such that$

$$
E\left[C_{r}^{(1)}\left(\psi_{n}\right)\right]=\xi\left(K_{1}, K_{2}, \ldots, K_{n-1}, K\right) .
$$

The arguments of this function will later be modified to obtain the costs for sub-cases A-(2) and A-(3), and for Case B.

When $\delta_{1}=\delta_{2}=\ldots=\delta_{n-2}=1$, this cost reduces to the one derived by Chukova et al. [13]; see page 75 for details.

Next, we use the function defined in equation (5.13) to define the costs for sub-cases A-(2) and A-(3).

(2): $\mathrm{r}_{1} \leq \mathrm{r} \leq \mathrm{r}_{2}$

Figure 5.3 depicts the case where $r_{1} \leq r \leq r_{2}$.

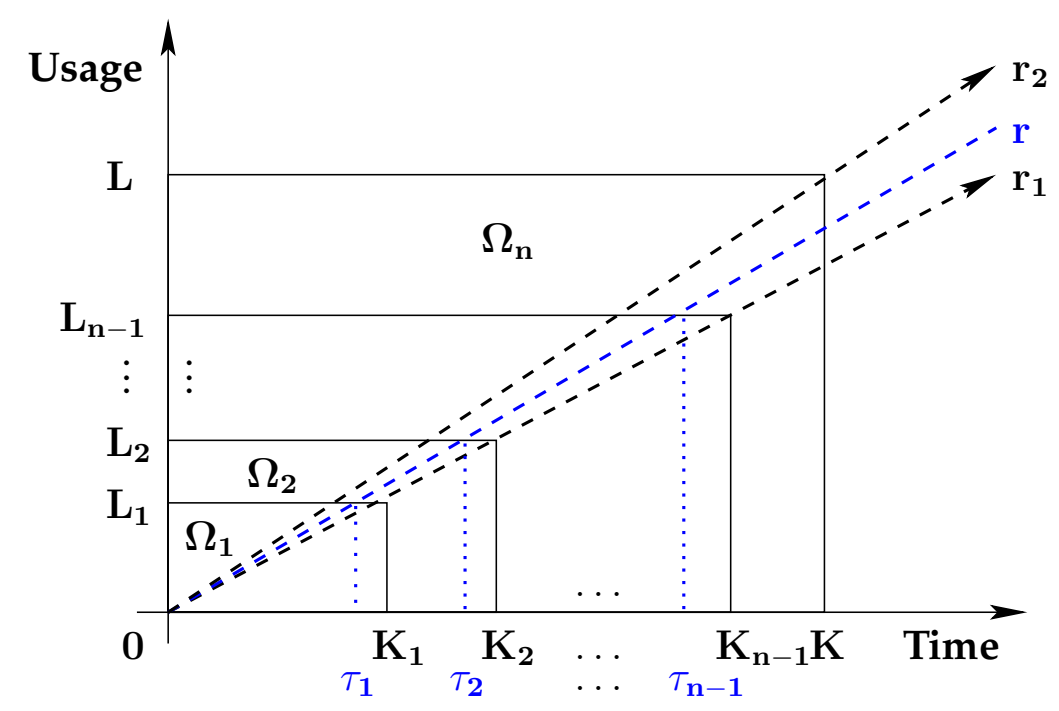

Figure 5.3: $r_{1} \leq r \leq r_{2}$.

When $r_{1} \leq r \leq r_{2}$, the warranty over the subregions $\Omega_{1}, \Omega_{2}, \ldots, \Omega_{n-1}$ will 
expire due to exceeding the usage limits $L_{1}, L_{2}, \ldots, L_{n-1}$ at time points

$$
\tau_{1}=\frac{L_{1}}{r}, \quad \tau_{2}=\frac{L_{2}}{r}, \quad \ldots, \quad \tau_{n-1}=\frac{L_{n-1}}{r},
$$

respectively. Therefore, the expected warranty cost for sub-case A-(2) is given by

$$
E\left[C_{r}^{(2)}\left(\psi_{n}\right)\right]=\xi\left(\tau_{1}, \tau_{2}, \ldots, \tau_{n-1}, K\right) .
$$

(3): $\mathrm{r}_{1} \leq \mathrm{r}_{2} \leq \mathrm{r}$

Figure 5.4 depicts the case where $r_{1} \leq r_{2} \leq r$. When $r_{1} \leq r_{2} \leq r$ the warranty over the entire region $\Omega$ expires at time

$$
\tau=\frac{L}{r}
$$

and the warranty over the subregions $\Omega_{1}, \Omega_{2}, \ldots, \Omega_{n-1}$ will expire at time points

$$
\tau_{1}=\frac{L_{1}}{r}, \quad \tau_{2}=\frac{L_{2}}{r}, \quad \ldots, \quad \tau_{n-1}=\frac{L_{n-1}}{r},
$$

respectively. Therefore, the expected warranty servicing cost for sub-case A-(3), is given by

$$
E\left[C_{r}^{(3)}\left(\psi_{n}\right)\right]=\xi\left(\tau_{1}, \tau_{2}, \ldots, \tau_{n-1}, \tau\right) .
$$

Finally, the expected total warranty cost for Case A, is derived by unconditioning the variable $R=r$. Therefore, for $r_{1} \leq r_{2}$, we have

$$
E\left[C_{A}^{\Omega}\left(\psi_{n}\right)\right]=\int_{0}^{r_{1}} E\left[C_{r}^{(1)}\left(\psi_{n}\right)\right] d G(r)+\int_{r_{1}}^{r_{2}} E\left[C_{r}^{(2)}\left(\psi_{n}\right)\right] d G(r)+\int_{r_{2}}^{\infty} E\left[C_{r}^{(3)}\left(\psi_{n}\right)\right] d G(r)
$$

where $G(r)$ is the distribution function of the usage rate $R$. 


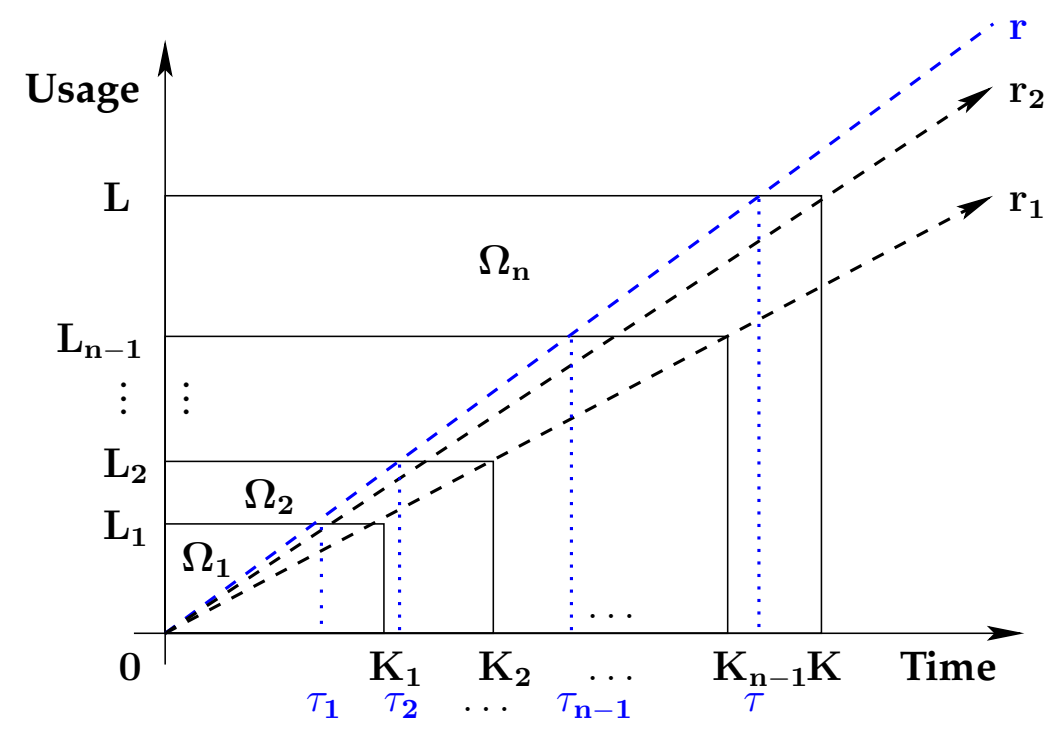

Figure 5.4: $r_{1} \leq r_{2} \leq r$.

\subsubsection{Case B: $r_{2} \leq r_{1}$}

For Case $\mathrm{B}$, as for Case $\mathrm{A}$, we conditional on the usage rate $R=r$ and derive the expected warranty servicing costs for the following three subcases:

$\begin{array}{ll}\text { (1) } & r \leq r_{2} \leq r_{1} \\ \text { (2) } & r_{2} \leq r \leq r_{1} \\ \text { (3) } & r_{2} \leq r_{1} \leq r\end{array}$

Let $E\left[C_{r}^{(1)}\left(\psi_{n}\right)\right], E\left[C_{r}^{(2)}\left(\psi_{n}\right)\right]$, and $E\left[C_{r}^{(3)}\left(\psi_{n}\right)\right]$ denote the expected warranty servicing costs, conditional on $R=r$, for the three sub-cases respectively [13]. Each of the expected costs $E\left[C_{r}^{(j)}\left(\psi_{n}\right)\right], j=1,2,3$, is the sum of the expected costs in each of the subregions $\Omega_{1}, \Omega_{2}, \ldots, \Omega_{n}$. That is

$$
E\left[C_{r}^{(j)}\left(\psi_{n}\right)=E\left[C_{r}^{\Omega_{1}}\left(\psi_{n}\right)\right]+E\left[C_{r}^{\Omega_{2}}\left(\psi_{n}\right)\right]+\ldots+E\left[C_{r}^{\Omega_{n}}\left(\psi_{n}\right)\right] .\right.
$$

We have not used the index $j$ in denoting the expected costs $E\left[C_{r}^{\Omega_{l}}\left(\psi_{n}\right)\right]$, where $l=1,2, \ldots, n$, since it is clear by the context that these costs are specific to the corresponding sub-cases of Case B. The expected total warranty 
servicing cost for Case B is similar to that of Case A in equation (5.14), with the following adjustments.

(1): $\mathrm{r} \leq \mathrm{r}_{2} \leq \mathrm{r}_{1}$

Figure 5.5 depicts the case where $r \leq r_{2} \leq r_{1}$.

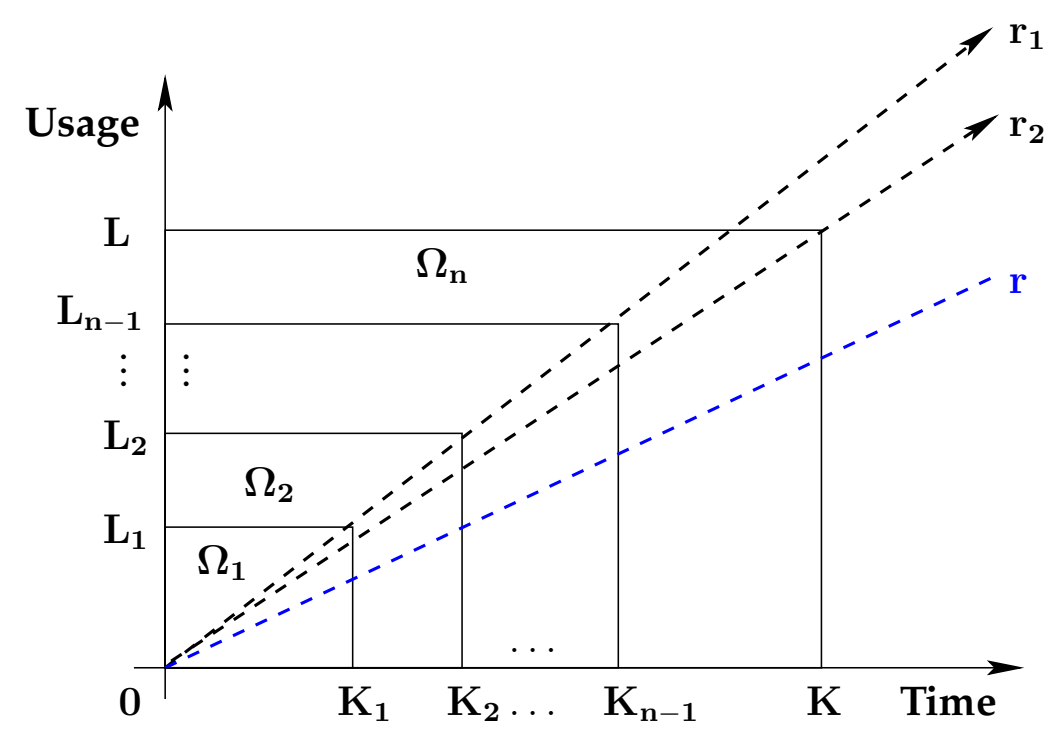

Figure 5.5: $r \leq r_{2} \leq r_{1}$

The expected warranty servicing cost for sub-case B-(1) is the same as that of sub-case A-(1), and is given by

$$
E\left[C_{r}^{(1)}\left(\psi_{n}\right)\right]=\xi\left(K_{1}, K_{2}, \ldots, K_{n-1}, K\right) .
$$

(2): $\mathrm{r}_{2} \leq \mathrm{r} \leq \mathrm{r}_{1}$

Figure 5.6 depicts the case where $r_{2} \leq r \leq r_{1}$.

The expected total warranty servicing cost for sub-case B-(2) is similar to that of sub-case B-(1), with the exception that the warranty over the entire region $\Omega$ expires at time

$$
\tau=\frac{L}{r} .
$$




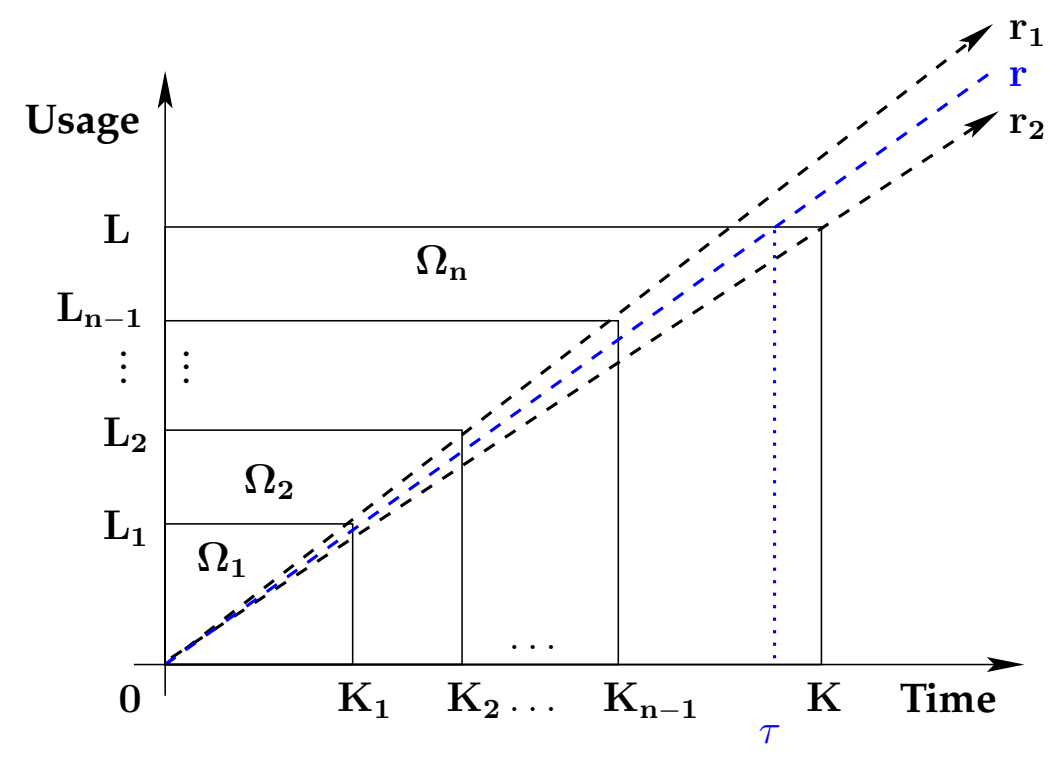

Figure 5.6: $r_{2} \leq r \leq r_{1}$

Therefore, the expected cost becomes

$$
E\left[C_{r}^{(2)}\left(\psi_{n}\right)\right]=\xi\left(K_{1}, K_{2}, \ldots, K_{n-1}, \tau\right) .
$$

(3): $\mathrm{r}_{2} \leq \mathrm{r}_{1} \leq \mathrm{r}$

Figure 5.7 depicts the case where $r_{2} \leq r_{1} \leq r$. The expected warranty servicing cost for sub-case B-(3) is the same as that of sub-case A-(3), where the warranty over the subregions $\Omega_{1}, \Omega_{2}, \ldots, \Omega_{n-1}, \Omega_{n}$ will expire at time points

$$
\tau_{1}=\frac{L_{1}}{r}, \quad \tau_{2}=\frac{L_{2}}{r}, \quad \ldots, \quad \tau_{n-1}=\frac{L_{n-1}}{r}, \quad \tau=\frac{L}{r},
$$

respectively. The expected total warranty servicing cost for sub-case B-(3) is given by

$$
E\left[C_{r}^{(3)}\left(\psi_{n}\right)\right]=\xi\left(\tau_{1}, \tau_{2}, \ldots, \tau_{n-1}, \tau\right) .
$$

Similar to the expected total warranty servicing cost for Case A, given in equation (5.14), unconditioning the variable $R=r$, we get the expected 


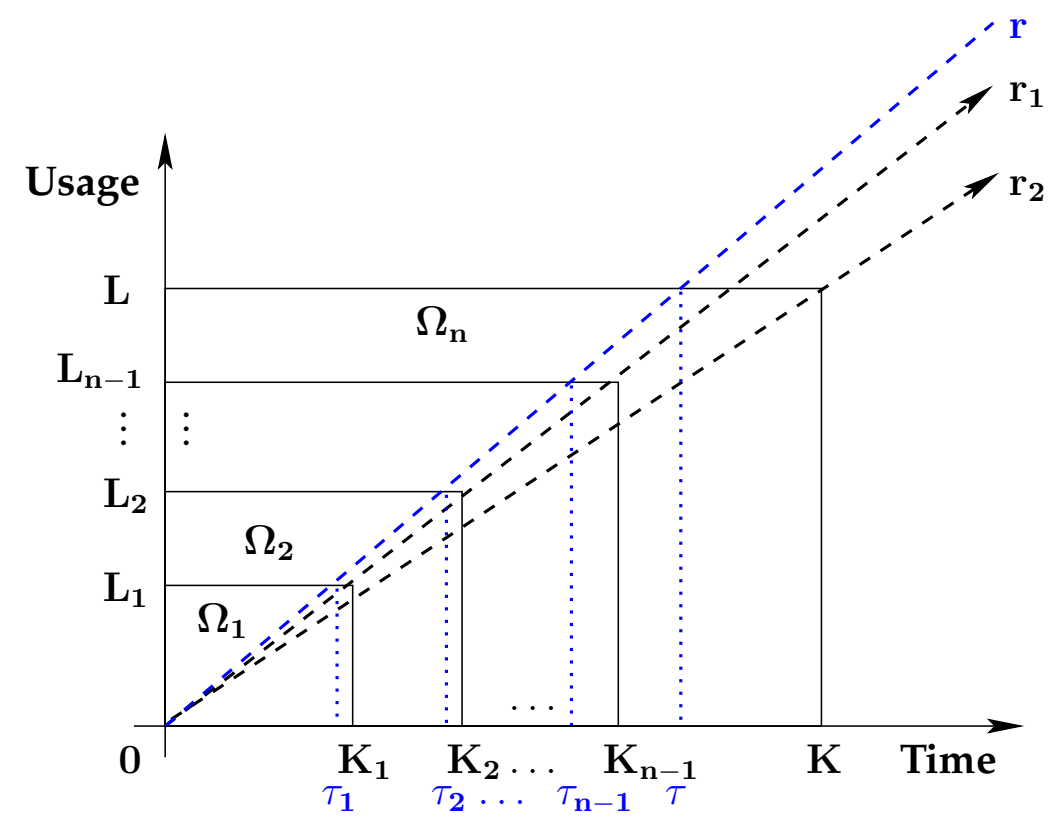

Figure 5.7: $r_{2} \leq r_{1} \leq r$

total warranty cost for Case B. That is $E\left[C_{B}^{\Omega}\left(\psi_{n}\right)\right]=\int_{0}^{r_{2}} E\left[C_{r}^{(1)}\left(\psi_{n}\right)\right] d G(r)+\int_{r_{2}}^{r_{1}} E\left[C_{r}^{(2)}\left(\psi_{n}\right)\right] d G(r)+\int_{r_{1}}^{\infty} E\left[C_{r}^{(3)}\left(\psi_{n}\right)\right] d G(r)$.

Numerical results illustrating the results derived in this chapter are presented in the following chapter. 


\section{Chapter 6}

\section{Numerical Illustration}

In this research, we study an imperfect repair strategy $\mathcal{S}_{n}^{\boldsymbol{\delta}}$ for a repairable product sold with a two-dimensional free-replacement warranty policy. The vector

$$
\boldsymbol{\delta}=\left(\delta_{1}, \delta_{2}, \ldots, \delta_{n-2}\right)
$$

denotes the degrees of the imperfect repairs, and $n$ denotes the number of subregions. The imperfect repair strategy is restricted and hence, characterized by $n$ decision variables

$$
\psi_{n}=\left(K_{1}, K_{2}, \ldots, K_{n-1}, r_{1}\right)
$$

The strategy is a generalized form of the repair-replacement strategy proposed by Chukova et al. [13] in which all repairs in the first and last subregions are minimal, and the first repair in each of the intermediate subregions is perfect (a replacement) while all consecutive repairs in the subregion are minimal. In the imperfect repair strategy considered here, the first repair in each of the intermediate subregions is imperfect, i.e. the degree of the repair is in the interval $(0,1)$. Refer to page 44 for a detailed description of the imperfect repair strategy. Assuming that the cost of an imperfect repair is proportional to the degree of the repair, with the imperfect repair strategy, we aim to reduce the warranty servicing cost by finding the set of decision variables that minimize the expected total warranty 
servicing cost over the rectangular warranty region $\Omega=[0, K) \times[0, L)$.

Two methods, described in Chapter 4 and Chapter 5, have been used to model the imperfect repairs, namely, the age reduction model in which the effect of the repair is characterized by a reduction in the virtual age of the product, and the intensity reduction model in which the effect of the repair is characterized by a reduction in the conditional intensity function of the failure process $[11,16]$. Having derived the expected total warranty servicing cost $E\left[C^{\Omega}\left(\psi_{n}\right)\right]$ for both models, under the restricted imperfect repair strategy $\mathcal{S}_{n}^{\boldsymbol{\delta}}$, we use numerical optimization to find the optimal

$$
\psi_{n}^{*}=\left(K_{1}^{*}, K_{2}^{*}, \ldots, K_{n-1}^{*}, r_{1}^{*}\right),
$$

which yields the minimum expected total warranty servicing $\operatorname{cost} E\left[C^{\Omega}\left(\psi_{n}^{*}\right)\right]$.

In this chapter, we provide numerical results for the imperfect repair strategies $\mathcal{S}_{3}^{\boldsymbol{\delta}}$, where the warranty region is divided into three subregions, and $\mathcal{S}_{4}^{\delta}$, where the warranty region is divided into four subregions, for both the age and intensity reduction models. For the 3-subregion strategy, there is one imperfect repair which is in the middle subregion, and for the 4 -subregion strategy, there are two imperfect repairs which are in the two middle subregions. The vector $\delta$ of degrees of the imperfect repairs for the two strategies are

$$
\boldsymbol{\delta}=\delta
$$

and

$$
\boldsymbol{\delta}=\left(\delta_{1}, \delta_{2}\right),
$$

respectively. For $r_{1} \leq r_{2}$, Figure 6.1 and Figure 6.2 depict the warranty region for $\mathcal{S}_{3}^{\boldsymbol{\delta}}$ and $\mathcal{S}_{4}^{\boldsymbol{\delta}}$, respectively.

For comparison reasons, we consider the example provided in the papers by Iskandar et al. [21] and Chukova et al. [13], and we use a numerical procedure (grid search) similar to the one used in these two papers. The details of the numerical example and search procedure are provided in the following section. 


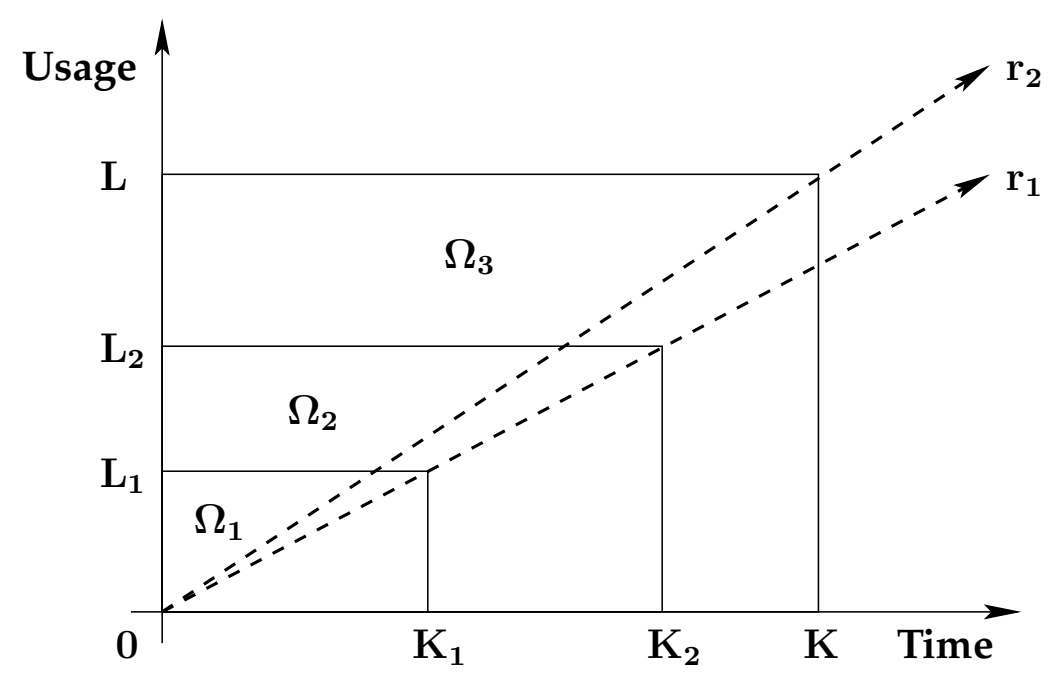

Figure 6.1: The 3-subregion rectangular warranty region

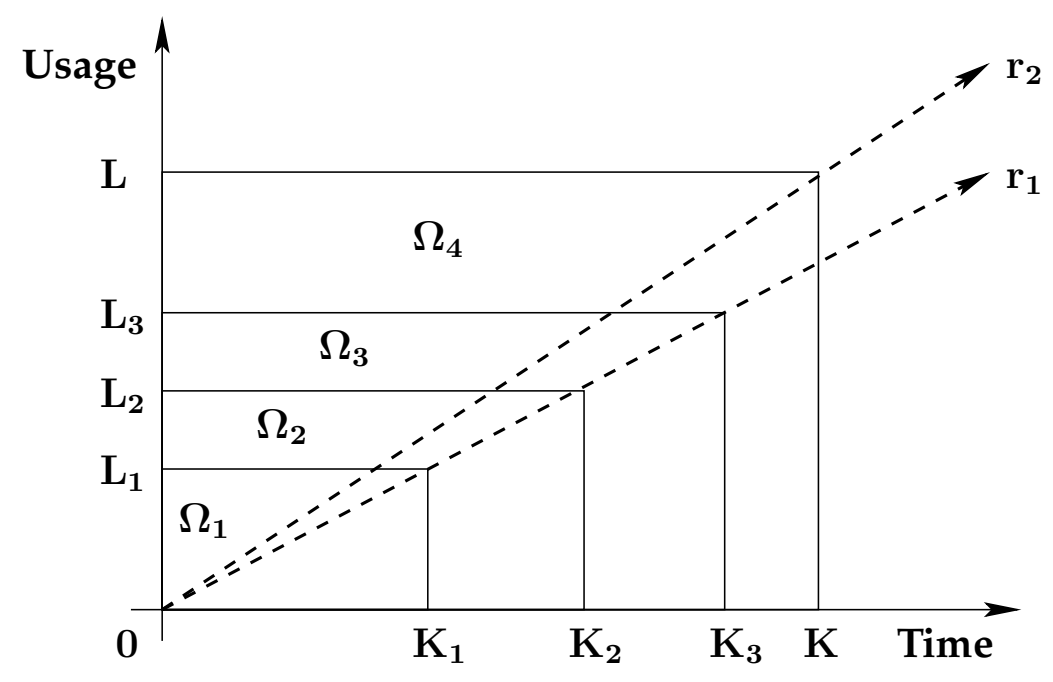

Figure 6.2: The 4-subregion rectangular warranty region

\subsection{Numerical Example}

The product under consideration is an automobile component sold with a free-replacement warranty. The time is measured in units of year and 
the usage is measured in units of 10,000 kilometers. It is assumed that $K=2$ and $L=2$, i.e. the time limit of the warranty policy is two years and the usage limit of the warranty policy is twenty thousand kilometers. Therefore,

$$
r_{2}=\frac{L}{K}=1
$$

For the 3 -subregion imperfect repair strategy $\mathcal{S}_{3}^{\boldsymbol{\delta}}$, in both the age and intensity reduction models, a grid search for minimizing the expected cost $E\left[C^{\Omega}\left(\psi_{3}\right)\right]$ was done with the decision variables $K_{1}$ and $K_{2}$ incremented in steps of 0.1 , over the interval $[0.1,2.0)$, and the decision variable $r_{1}$ incremented in steps of 0.2 , starting at 0.2 . For the 4 -subregion imperfect repair strategy $\mathcal{S}_{4}^{\boldsymbol{\delta}}$, in both the age and intensity reduction models, a grid search for minimizing the expected cost $E\left[C^{\Omega}\left(\psi_{4}\right)\right]$ was done with the decision variables $K_{1}, K_{2}$ and $K_{3}$ incremented in steps of 0.1 , over the interval $[0.1,2.0)$, and the decision variable $r_{1}$ incremented in steps of 0.2 , starting at $0.2[21]$.

\subsubsection{Repair Costs}

Let $c_{\text {min }}, c_{i m p}$ and $c_{p e r}$ denote the costs of a minimal repair, an imperfect repair and a perfect repair (replacement) respectively. The costs $c_{\min }$ and $c_{\text {per }}$ are assumed to be constant, and the $\operatorname{cost} c_{i m p}$ is assumed to be proportional to the degree of repair. We also assume that

$$
c_{\text {min }}<c_{i m p}<c_{p e r} .
$$

For the numerical example, we assume that the degrees of the imperfect repairs in all the intermediate subregions are equal. That is, for $\mathcal{S}_{4}^{\boldsymbol{\delta}}$ where we have two intermediate subregions and hence, two possible imperfect repairs with degrees $\delta_{1}$ and $\delta_{2}$ respectively, we assume that $\delta_{1}=\delta_{2}=\delta$. Hence, $\boldsymbol{\delta}=\delta$. This makes $\mathcal{S}_{4}^{\boldsymbol{\delta}}$ comparable with $\mathcal{S}_{3}^{\boldsymbol{\delta}}$ when the values of $\delta$ are equal. We define the ratios

$$
\mu=\frac{c_{\min }}{c_{p e r}}
$$


and

$$
\delta=\frac{c_{i m p}}{c_{p e r}}
$$

and compute the expected warranty servicing costs $E\left[C^{\Omega}\left(\psi_{3}\right)\right]$ and $E\left[C^{\Omega}\left(\psi_{4}\right)\right]$ for different values of $\mu$ and $\delta$ for both imperfect repair models. In this numerical example, as suggested by Iskandar et al [21], we set $c_{\text {per }}=1$, which makes the ratios $\mu$ and $\delta$ the cost of a minimal repair and the cost of an imperfect repair, respectively.

\subsubsection{Initial Intensity Function}

The initial intensity function, conditional on $R=r$, is of the form

$$
\lambda(t \mid r)=\theta_{0}+\theta_{1} r+\theta_{2} A^{2}(t)+\theta_{3} A(t) U(t),
$$

where $\theta_{0}, \theta_{1}, \theta_{2}$, and $\theta_{3}$ are all positive constants [6]. Since usage is a function of age, this intensity function reduces to

$$
\lambda(t \mid r)=\theta_{0}+\theta_{1} r+\left(\theta_{2}+\theta_{3} r\right) A^{2}(t) .
$$

As long as the failed product is repaired minimally, this function further reduces to

$$
\lambda(t \mid r)=\theta_{0}+\theta_{1} r+\left(\theta_{2}+\theta_{3} r\right) t^{2},
$$

making the cumulative intensity function before the first imperfect repair

$$
\begin{aligned}
\Lambda(t \mid r) & =\int_{0}^{t} \lambda(s \mid r) d s \\
& =\theta_{0} t+\theta_{1} r t+\left(\theta_{2}+\theta_{3} r\right) \frac{t^{3}}{3} .
\end{aligned}
$$

As in the example by Iskandar et al. [21], here, the values of the parameters are $\theta_{0}=0.1, \theta_{1}=0.2, \theta_{2}=0.7$ and $\theta_{3}=0.7$. Therefore, equation (6.1) becomes

$$
\lambda(t \mid r)=0.1+0.2 r+(0.7+0.7 r) t^{2},
$$


and the cumulative intensity function in equation (6.2) becomes

$$
\Lambda(t \mid r)=0.1 t+0.2 r t+(0.7+0.7 r) \frac{t^{3}}{3} \text {. }
$$

These functions are conditional on the usage rate $R$. In order to determine the intensity function and hence, the unconditional expected costs, we need to remove the conditioning on $R=r$.

\subsubsection{Distribution of the Usage Rate $\mathbf{R}$}

The usage rate $R$ is uniformly distributed over an interval $\left[r_{l}, r_{u}\right]$ with distribution and density functions

$$
\begin{aligned}
& G(r)= \begin{cases}\frac{r-r_{l}}{r_{u}-r_{l}}, & r \in\left[r_{l}, r_{u}\right] \\
0, & \text { otherwise }\end{cases} \\
& g(r)= \begin{cases}\frac{1}{r_{u}-r_{l}}, & r \in\left[r_{l}, r_{u}\right] \\
0, & \text { otherwise }\end{cases}
\end{aligned}
$$

respectively.

In the numerical example, three usage categories are considered for the consumer usage rate $R$ : light, medium and heavy [21]. The distribution functions corresponding to the three categories are

$$
\begin{array}{ll}
\text { Light: } & G(r)= \begin{cases}\frac{r-0.1}{0.9-0.1}, & r \in[0.1,0.9] \\
0, & \text { otherwise },\end{cases} \\
\text { Medium: } & G(r)= \begin{cases}\frac{r-0.7}{1.3-0.7}, & r \in[0.7,1.3] \\
0, & \text { otherwise }\end{cases} \\
\text { Heavy : } & G(r)= \begin{cases}\frac{r-1.1}{2.9-1.1}, & r \in[1.1,2.9] \\
0, & \text { otherwise }\end{cases}
\end{array}
$$

Since, the usage rate $R$ is uniformly distributed over $\left[r_{l}, r_{u}\right]$, the expected usage rate across the population of consumers is given by

$$
E(R)=\frac{1}{r_{u}-r_{l}} \int_{r_{l}}^{r_{u}} r d r=\frac{r_{l}+r_{u}}{2} .
$$




\subsubsection{Time and Usage at First Failure}

The expected time to first failure of the product is given by

$$
\begin{aligned}
E\left(T_{1}\right) & =\int_{r_{l}}^{r_{u}}\left\{\int_{0}^{\infty} \bar{F}_{T_{1 \mid r}}(t) d t\right\} d G(r) \\
& =\frac{1}{r_{u}-r_{l}} \int_{r_{l}}^{r_{u}} \int_{0}^{\infty} \exp \left\{-\int_{0}^{t}\left[\theta_{0}+\theta_{1} r+\left(\theta_{2}+\theta_{3} r\right) s^{2}\right] d s\right\} d t d r .
\end{aligned}
$$

where $\bar{F}_{T_{1 \mid r}}(t)$ is the probability that the first failure after $K_{1}$ is after $t$. Equation (6.3) can be solved numerically. The usage rate $R$ and the time to first failure $T_{1}$ are independent. Hence, the expected usage at first failure $E\left(U_{1}\right)$ is the product of the expected usage rate $E(R)$ and the expected time to first failure $E\left(T_{1}\right)$, i.e.

$$
E\left(U_{1}\right)=E(R) E\left(T_{1}\right) .
$$

For the numerical example considered, the expected usage rate, the expected time to first failure and the expected usage at first failure are presented in Table 6.1.

Table 6.1: Expected time and usage at first failure

\begin{tabular}{|c|c|c|c|c|}
\hline Usage type & {$\left[\mathbf{r}_{\mathbf{l}}, \mathbf{r}_{\mathbf{u}}\right]$} & $\mathbf{E}(\mathbf{R})$ & $\mathbf{E}\left(\mathbf{T}_{\mathbf{1}}\right)$ & $\mathbf{E}\left(\mathbf{U}_{\mathbf{1}}\right)$ \\
\hline Light & {$[0.1,0.9]$} & 0.5 & 1.1118 & 0.5559 \\
Medium & {$[0.7,1.3]$} & 1.0 & 0.9575 & 0.9575 \\
Heavy & {$[1.1,2.9]$} & 2.0 & 0.7755 & 1.5510 \\
\hline
\end{tabular}

Hence, the time to first failure for the light usage category is 1.1118 years and the corresponding usage at first failure is 5559 kilometers; the time to first failure for the medium usage category is 0.9575 years and the corresponding usage at first failure is 9575 kilometers; the time to first failure 
for the heavy usage category is 0.7755 years and the corresponding usage at first failure is 15,510 kilometers.

\subsection{Results for Age Reduction Model}

The expected total warranty servicing cost $E\left[C^{\Omega}\left(\psi_{n}\right)\right]$, for the age reduction model is given by equation (4.21) (on page 77) when $r_{1} \leq r_{2}$ and equation (4.22) (on page 80) when $r_{2} \leq r_{1}$.

\subsubsection{Expected Costs for the 3-Subregion Strategy}

For strategy $\mathcal{S}_{3}^{\boldsymbol{\delta}}$, when $r_{1} \leq r_{2}$, the expected warranty servicing cost $E\left[C^{\Omega}\left(\psi_{3}\right)\right]$ is given by

$$
E\left[C_{A}^{\Omega}\left(\psi_{3}\right)\right]=\int_{0}^{r_{1}} E\left[C_{r}^{(1)}\left(\psi_{3}\right)\right] d G(r)+\int_{r_{1}}^{r_{2}} E\left[C_{r}^{(2)}\left(\psi_{3}\right)\right] d G(r)+\int_{r_{2}}^{\infty} E\left[C_{r}^{(3)}\left(\psi_{3}\right)\right] d G(r)
$$

where

$$
\begin{aligned}
E\left[C_{r}^{(1)}\left(\psi_{3}\right)\right]= & c_{\min } \Lambda\left(K_{1} \mid r\right) \\
+ & c_{\min }\left[\Lambda(K \mid r)-\Lambda\left(K_{2} \mid r\right)\right] e^{-\left[\Lambda\left(K_{2} \mid r\right)-\Lambda\left(K_{1} \mid r\right)\right]} \\
+ & \int_{K_{1}}^{K_{2}}\left\{\left(c_{i m p}+c_{\min }\left\{\Lambda\left[A_{1}\left(K_{2}\right) \mid r\right]-\Lambda\left[A_{1}\left(u_{1}\right) \mid r\right]\right\}\right.\right. \\
& \left.\left.\quad+c_{\min }\left\{\Lambda\left[A_{1}(K) \mid r\right]-\Lambda\left[A_{1}\left(K_{2}\right) \mid r\right]\right\}\right) f_{T_{K_{1} \mid r}}\left(u_{1}\right)\right\} d u_{1} \\
= & \zeta\left(K_{1}, K_{2}, K\right)
\end{aligned}
$$

is the cost when $r \leq r_{1} \leq r_{2}$,

$$
E\left[C_{r}^{(2)}\left(\psi_{3}\right)\right]=\zeta\left(\tau_{1}, \tau_{2}, K\right)
$$

is the cost when $r_{1} \leq r \leq r_{2}$, and

$$
E\left[C_{r}^{(3)}\left(\psi_{3}\right)\right]=\zeta\left(\tau_{1}, \tau_{2}, \tau\right)
$$


is the cost when $r_{1} \leq r_{2} \leq r$. When $r_{2} \leq r_{1}$, the expected warranty servicing cost $E\left[C^{\Omega}\left(\psi_{3}\right)\right]$ is given by $E\left[C_{B}^{\Omega}\left(\psi_{3}\right)\right]=\int_{0}^{r_{2}} E\left[C_{r}^{(1)}\left(\psi_{3}\right)\right] d G(r)+\int_{r_{2}}^{r_{1}} E\left[C_{r}^{(2)}\left(\psi_{3}\right)\right] d G(r)+\int_{r_{1}}^{\infty} E\left[C_{r}^{(3)}\left(\psi_{3}\right)\right] d G(r)$,

where

$$
E\left[C_{r}^{(1)}\left(\psi_{3}\right)\right]=\zeta\left(K_{1}, K_{2}, K\right)
$$

is the cost when $r \leq r_{2} \leq r_{1}$,

$$
E\left[C_{r}^{(2)}\left(\psi_{3}\right)\right]=\zeta\left(K_{1}, K_{2}, \tau\right)
$$

is the cost when $r_{2} \leq r \leq r_{1}$, and

$$
E\left[C_{r}^{(3)}\left(\psi_{3}\right)\right]=\zeta\left(\tau_{1}, \tau_{2}, \tau\right)
$$

is the cost when $r_{2} \leq r_{1} \leq r$; refer to Chapter 4 for more information.

Table 6.2 provides the minimum expected total warranty servicing cost $E\left[C^{\Omega}\left(\psi_{3}^{*}\right)\right]$, with the optimal partition

$$
\psi_{3}^{*}=\left(K_{1}^{*}, K_{2}^{*}, r_{1}^{*}\right)
$$

for different values of $\mu$ and $\delta$, for the light usage category. The structure of the table is as follows: the ratio $\mu$ of the cost of a minimal repair to the cost of a perfect repair is displayed in the first column, the degree $\delta$ of the imperfect repair, which is also the ratio of the cost of an imperfect repair to the cost of a perfect repair, corresponding to our strategy is displayed in the second column, the optimal value of the decision variables $K_{1}^{*}, K_{2}^{*}$ and $r_{1}^{*}$ are given in the next three columns, the corresponding expected cost $E\left[C^{\Omega}\left(\psi_{3}^{*}\right)\right]$ is given in the sixth column, the expected cost $E\left[C^{\Omega}\left(\hat{\phi}_{3}{ }^{*}\right)\right]$ for the 3-subregion restricted repair-replacement strategy by Iskandar et al. [21] is displayed in the seventh column, the expected cost $E\left[C^{\Omega}\left(\gamma_{3}^{*}\right)\right]$ for the 3-subregion unrestricted repair-replacement strategy by Chukova and Johnston [14] is provided in the eighth column, and the expected cost 
$E\left[C_{M R}^{\Omega}\left(\gamma^{*}\right)\right]$ for an all minimal repair strategy is presented in the last column. The expected cost $E\left[C^{\Omega}\left(\psi_{3}^{*}\right)\right]$ corresponding to the imperfect repair strategy is printed in boldface if it is the minimum for a given value of $\mu$.

We observe, in Table 6.2 (light usage category), that for $\mu=0.1$, the strategy that costs the least is an all minimal repair strategy; when $\mu=0.2$, there are two imperfect repair strategies that cost the least compared to other strategies; when $\mu>0.2$, the imperfect repair strategies all yield lower costs than the other strategies with the strategy corresponding to the lowest degree of repair $\delta, \mu<\delta<1.0$, being the best for a given value of the ratio $\mu$.

Similarly, Table 6.3 and Table 6.4 provide the minimum expected total warranty servicing cost $E\left[C^{\Omega}\left(\psi_{3}^{*}\right)\right]$ for the medium and heavy usage categories respectively; all three tables have the same structure, and as before, the expected $\cos t E\left[C^{\Omega}\left(\psi_{3}^{*}\right)\right]$ corresponding to the imperfect repair strategy is printed in boldface if it is the minimum of the costs for a given value of the ratio $\mu$.

For the medium usage category (Table 6.3), as in the light category, for $\mu=0.1$, the strategy that costs the least is the all minimal repair strategy and for $\mu=0.2$, two of the imperfect repair strategies cost lower than the corresponding restricted and unrestricted repair-replacement strategies and the all minimal repair strategy. For $\mu>0.2$, the imperfect repair strategies cost lower than the corresponding alternate strategies.

For the heavy usage category (Table 6.4), for $\mu=0.1,0.2$, the strategy that costs the least is the all minimal repair strategy and for $\mu=0.3$, the imperfect repair strategy with $\delta=0.4$ costs lower than the corresponding restricted and unrestricted repair-replacement strategies and the all minimal repair strategy. For $\mu>0.3$, the imperfect repair strategies cost lower than the corresponding alternate replacement and minimal repair strategies; the strategy with the smallest $\delta, \mu<\delta<1.0$, yields the lowest cost among the imperfect repair strategies. 
Table 6.2: Age reduction $\mathcal{S}_{3}^{\delta}$ : costs for light usage

\begin{tabular}{|c|c|c|c|c|c|c|c|c|}
\hline$\mu$ & $\delta$ & $\mathbf{K}_{1}^{*}$ & $\mathbf{K}_{2}^{*}$ & $\mathrm{r}_{1}^{*}$ & $\mathbf{E}\left[\mathbf{C}^{\boldsymbol{\Omega}}\left(\psi_{\mathbf{3}}^{*}\right)\right]$ & $\mathbf{E}\left[\mathbf{C}^{\boldsymbol{\Omega}}\left({\hat{\phi_{3}}}^{*}\right)\right]$ & $\mathbf{E}\left[\mathbf{C}^{\boldsymbol{\Omega}}\left(\gamma_{\mathbf{3}}^{*}\right)\right]$ & $\mathbf{E}\left[\mathbf{C}_{\mathbf{M R}}^{\Omega}\left(\gamma^{*}\right)\right]$ \\
\hline \multirow{8}{*}{0.1} & 0.2 & 0.1 & 0.2 & 0.2 & 0.3209 & \multirow{8}{*}{0.3281} & \multirow{8}{*}{0.3231} & \multirow{8}{*}{0.3200} \\
\hline & 0.3 & 0.1 & 0.2 & 0.2 & 0.3218 & & & \\
\hline & 0.4 & 0.1 & 0.2 & 0.2 & 0.3227 & & & \\
\hline & 0.5 & 0.1 & 0.2 & 0.2 & 0.3236 & & & \\
\hline & 0.6 & 0.1 & 0.2 & 0.2 & 0.3245 & & & \\
\hline & 0.7 & 0.1 & 0.2 & 0.2 & 0.3254 & & & \\
\hline & 0.8 & 0.1 & 0.2 & 0.2 & 0.3263 & & & \\
\hline & 0.9 & 0.1 & 0.2 & 0.2 & 0.3272 & & & \\
\hline \multirow{7}{*}{0.2} & 0.3 & 0.8 & 1.7 & 1.0 & 0.5908 & \multirow{7}{*}{0.6469} & \multirow{7}{*}{0.6427} & \multirow{7}{*}{0.6400} \\
\hline & 0.4 & 0.9 & 1.4 & 1.0 & 0.6292 & & & \\
\hline & 0.5 & 0.1 & 0.2 & 0.2 & 0.6425 & & & \\
\hline & 0.6 & 0.1 & 0.2 & 0.2 & 0.6434 & & & \\
\hline & 0.7 & 0.1 & 0.2 & 0.2 & 0.6443 & & & \\
\hline & 0.8 & 0.1 & 0.2 & 0.2 & 0.6451 & & & \\
\hline & 0.9 & 0.1 & 0.2 & 0.2 & 0.6460 & & & \\
\hline \multirow{6}{*}{0.3} & 0.4 & 0.7 & 1.9 & 1.0 & 0.7863 & \multirow{6}{*}{0.9656} & \multirow{6}{*}{0.9623} & \multirow{6}{*}{0.9600} \\
\hline & 0.5 & 0.7 & 1.7 & 1.0 & 0.8226 & & & \\
\hline & 0.6 & 0.7 & 1.6 & 1.0 & 0.8602 & & & \\
\hline & 0.7 & 0.7 & 1.5 & 1.0 & 0.8979 & & & \\
\hline & 0.8 & 0.8 & 1.4 & 1.0 & 0.9330 & & & \\
\hline & 0.9 & 0.9 & 1.1 & 1.0 & 0.9585 & & & \\
\hline \multirow{5}{*}{0.4} & 0.5 & 0.7 & 1.9 & 1.0 & 0.9488 & \multirow{5}{*}{1.1401} & \multirow{5}{*}{1.1400} & \multirow{5}{*}{1.2800} \\
\hline & 0.6 & 0.7 & 1.8 & 1.0 & 0.9781 & & & \\
\hline & 0.7 & 0.7 & 1.8 & 1.0 & 1.0123 & & & \\
\hline & 0.8 & 0.7 & 1.7 & 1.0 & 1.0511 & & & \\
\hline & 0.9 & 0.7 & 1.6 & 1.0 & 1.0942 & & & \\
\hline \multirow{4}{*}{0.5} & 0.6 & 0.6 & 1.9 & 1.0 & 1.0859 & \multirow{4}{*}{1.2258} & \multirow{4}{*}{1.2258} & \multirow{4}{*}{1.6000} \\
\hline & 0.7 & 0.6 & 1.9 & 1.0 & 1.1093 & & & \\
\hline & 0.8 & 0.6 & 1.8 & 1.0 & 1.1410 & & & \\
\hline & 0.9 & 0.6 & 1.8 & 1.0 & 1.1799 & & & \\
\hline \multirow{3}{*}{0.6} & 0.7 & 0.6 & 1.9 & 1.0 & 1.2027 & \multirow{3}{*}{1.2971} & \multirow{3}{*}{1.2971} & \multirow{3}{*}{1.9200} \\
\hline & 0.8 & 0.6 & 1.9 & 1.0 & 1.2236 & & & \\
\hline & 0.9 & 0.6 & 1.9 & 1.0 & 1.2558 & & & \\
\hline \multirow{2}{*}{0.7} & 0.8 & 0.6 & 1.9 & 1.0 & 1.3052 & \multirow{2}{*}{1.3627} & 13627 & 22400 \\
\hline & 0.9 & 0.6 & 1.9 & 1.0 & 1.3274 & & 1.0627 & $2 . \angle 400$ \\
\hline 0.8 & 0.9 & 0.6 & 1.9 & 1.0 & 1.3990 & 1.4263 & 1.4263 & 2.5600 \\
\hline
\end{tabular}


Table 6.3: Age reduction $\mathcal{S}_{3}^{\delta}$ : costs for medium usage

\begin{tabular}{|c|c|c|c|c|c|c|c|c|}
\hline$\mu$ & $\delta$ & $\mathbf{K}_{1}^{*}$ & $\mathbf{K}_{2}^{*}$ & $\mathrm{r}_{1}^{*}$ & $\mathbf{E}\left[\mathbf{C}^{\boldsymbol{\Omega}}\left(\psi_{\mathbf{3}}^{*}\right)\right]$ & $\mathbf{E}\left[\mathbf{C}^{\boldsymbol{\Omega}}\left({\hat{\phi_{3}}}^{*}\right)\right]$ & $\mathbf{E}\left[\mathbf{C}^{\boldsymbol{\Omega}}\left(\gamma_{\mathbf{3}}^{*}\right)\right]$ & $\mathbf{E}\left[\mathbf{C}_{\mathbf{M R}}^{\boldsymbol{\Omega}}\left(\gamma^{*}\right)\right]$ \\
\hline \multirow{8}{*}{0.1} & 0.2 & 0.5 & 0.6 & 0.2 & 0.3643 & \multirow{8}{*}{0.3691} & \multirow{8}{*}{0.3637} & \multirow{8}{*}{0.3637} \\
\hline & 0.3 & 0.3 & 0.4 & 0.2 & 0.3649 & & & \\
\hline & 0.4 & 0.3 & 0.4 & 0.2 & 0.3655 & & & \\
\hline & 0.5 & 0.3 & 0.4 & 0.2 & 0.3661 & & & \\
\hline & 0.6 & 0.2 & 0.3 & 0.2 & 0.3667 & & & \\
\hline & 0.7 & 0.2 & 0.3 & 0.2 & 0.3673 & & & \\
\hline & 0.8 & 0.2 & 0.3 & 0.2 & 0.3679 & & & \\
\hline & 0.9 & 0.2 & 0.3 & 0.2 & 0.3685 & & & \\
\hline \multirow{7}{*}{0.2} & 0.3 & 0.8 & 1.7 & 1.0 & 0.6616 & \multirow{7}{*}{0.7319} & \multirow{7}{*}{0.7274} & \multirow{7}{*}{0.7274} \\
\hline & 0.4 & 0.8 & 1.5 & 1.0 & 0.7013 & & & \\
\hline & 0.5 & 1.2 & 1.3 & 0.8 & 0.7276 & & & \\
\hline & 0.6 & 1.4 & 1.5 & 0.2 & 0.7295 & & & \\
\hline & 0.7 & 0.9 & 1.0 & 0.2 & 0.7301 & & & \\
\hline & 0.8 & 0.8 & 0.9 & 0.2 & 0.7307 & & & \\
\hline & 0.9 & 0.7 & 0.8 & 0.2 & 0.7313 & & & \\
\hline \multirow{6}{*}{0.3} & 0.4 & 0.7 & 1.9 & 1.0 & 0.8819 & \multirow{6}{*}{1.0894} & \multirow{6}{*}{1.0793} & \multirow{6}{*}{1.0911} \\
\hline & 0.5 & 0.7 & 1.8 & 1.0 & 0.9139 & & & \\
\hline & 0.6 & 0.7 & 1.7 & 1.0 & 0.9493 & & & \\
\hline & 0.7 & 0.7 & 1.6 & 1.0 & 0.9874 & & & \\
\hline & 0.8 & 0.8 & 1.5 & 1.0 & 1.0265 & & & \\
\hline & 0.9 & 0.8 & 1.3 & 1.0 & 1.0628 & & & \\
\hline \multirow{5}{*}{0.4} & 0.5 & 0.7 & 1.9 & 1.0 & 1.0653 & \multirow{5}{*}{1.2420} & \multirow{5}{*}{1.2417} & \multirow{5}{*}{1.4549} \\
\hline & 0.6 & 0.7 & 1.9 & 1.0 & 1.0881 & & & \\
\hline & 0.7 & 0.7 & 1.8 & 1.0 & 1.1170 & & & \\
\hline & 0.8 & 0.7 & 1.7 & 1.0 & 1.1532 & & & \\
\hline & 0.9 & 0.7 & 1.7 & 1.0 & 1.1955 & & & \\
\hline \multirow{4}{*}{0.5} & 0.6 & 0.7 & 1.9 & 1.0 & 1.2197 & \multirow{4}{*}{1.3390} & \multirow{4}{*}{1.3390} & \multirow{4}{*}{1.8186} \\
\hline & 0.7 & 0.7 & 1.9 & 1.0 & 1.2356 & & & \\
\hline & 0.8 & 0.6 & 1.8 & 1.0 & 1.2617 & & & \\
\hline & 0.9 & 0.6 & 1.8 & 1.0 & 1.2954 & & & \\
\hline \multirow{3}{*}{0.6} & 0.7 & 0.6 & 1.9 & 1.0 & 1.3517 & \multirow{3}{*}{1.4249} & \multirow{3}{*}{1.4249} & \multirow{3}{*}{2.1823} \\
\hline & 0.8 & 0.6 & 1.9 & 1.0 & 1.3638 & & & \\
\hline & 0.9 & 0.6 & 1.9 & 1.0 & 1.3885 & & & \\
\hline \multirow{2}{*}{0.7} & 0.8 & 0.6 & 1.9 & 1.0 & 1.4658 & \multirow{2}{*}{1.5066} & 15066 & 25460 \\
\hline & 0.9 & 0.6 & 1.9 & 1.0 & 1.4789 & & 1.0000 & 2.5400 \\
\hline 0.8 & 0.9 & 0.6 & 1.9 & 1.0 & 1.5693 & 1.5875 & 1.5875 & 2.9097 \\
\hline
\end{tabular}


Table 6.4: Age reduction $\mathcal{S}_{3}^{\delta}$ : costs for heavy usage

\begin{tabular}{|c|c|c|c|c|c|c|c|c|}
\hline$\mu$ & $\delta$ & $\mathbf{K}_{1}^{*}$ & $\mathbf{K}_{2}^{*}$ & $\mathrm{r}_{1}^{*}$ & $\mathbf{E}\left[\mathbf{C}^{\boldsymbol{\Omega}}\left(\psi_{\mathbf{3}}^{*}\right)\right]$ & $\mathbf{E}\left[\mathbf{C}^{\boldsymbol{\Omega}}\left({\hat{\phi_{3}}}^{*}\right)\right]$ & $\mathbf{E}\left[\mathbf{C}^{\boldsymbol{\Omega}}\left(\gamma_{\mathbf{3}}^{*}\right)\right]$ & $\mathbf{E}\left[\mathbf{C}_{\mathbf{M R}}^{\Omega}\left(\gamma^{*}\right)\right]$ \\
\hline \multirow{8}{*}{0.1} & 0.2 & 0.5 & 0.6 & 0.2 & 0.1465 & \multirow{8}{*}{0.1505} & \multirow{8}{*}{0.1466} & \multirow{8}{*}{0.1460} \\
\hline & 0.3 & 0.4 & 0.5 & 0.2 & 0.1470 & & & \\
\hline & 0.4 & 0.3 & 0.4 & 0.2 & 0.1475 & & & \\
\hline & 0.5 & 0.3 & 0.4 & 0.2 & 0.1480 & & & \\
\hline & 0.6 & 0.3 & 0.4 & 0.2 & 0.1485 & & & \\
\hline & 0.7 & 0.3 & 0.4 & 0.2 & 0.1490 & & & \\
\hline & 0.8 & 0.2 & 0.3 & 0.2 & 0.1495 & & & \\
\hline & 0.9 & 0.2 & 0.3 & 0.2 & 0.1500 & & & \\
\hline \multirow{7}{*}{0.2} & 0.3 & 1.8 & 1.9 & 0.2 & 0.2924 & \multirow{7}{*}{0.2959} & \multirow{7}{*}{0.2924} & \multirow{7}{*}{0.2919} \\
\hline & 0.4 & 1.3 & 1.4 & 0.2 & 0.2929 & & & \\
\hline & 0.5 & 0.9 & 1.0 & 0.2 & 0.2934 & & & \\
\hline & 0.6 & 0.8 & 0.9 & 0.2 & 0.2939 & & & \\
\hline & 0.7 & 0.7 & 0.8 & 0.2 & 0.2944 & & & \\
\hline & 0.8 & 0.7 & 0.8 & 0.2 & 0.2949 & & & \\
\hline & 0.9 & 0.6 & 0.7 & 0.2 & 0.2954 & & & \\
\hline \multirow{6}{*}{0.3} & 0.4 & 1.0 & 1.9 & 0.8 & 0.4251 & \multirow{6}{*}{0.4412} & \multirow{6}{*}{0.4381} & \multirow{6}{*}{0.4379} \\
\hline & 0.5 & 1.8 & 1.9 & 0.2 & 0.4387 & & & \\
\hline & 0.6 & 1.8 & 1.9 & 0.2 & 0.4392 & & & \\
\hline & 0.7 & 1.8 & 1.9 & 0.2 & 0.4397 & & & \\
\hline & 0.8 & 1.5 & 1.6 & 0.2 & 0.4402 & & & \\
\hline & 0.9 & 1.3 & 1.4 & 0.2 & 0.4407 & & & \\
\hline \multirow{5}{*}{0.4} & 0.5 & 0.6 & 1.7 & 1.0 & 0.5348 & \multirow{5}{*}{0.5864} & \multirow{5}{*}{0.5817} & \multirow{5}{*}{0.5839} \\
\hline & 0.6 & 0.8 & 1.5 & 1.0 & 0.5672 & & & \\
\hline & 0.7 & 1.8 & 1.9 & 0.6 & 0.5838 & & & \\
\hline & 0.8 & 1.8 & 1.9 & 0.2 & 0.5854 & & & \\
\hline & 0.9 & 1.8 & 1.9 & 0.2 & 0.5859 & & & \\
\hline \multirow{4}{*}{0.5} & 0.6 & 0.6 & 1.8 & 1.0 & 0.6346 & \multirow{4}{*}{0.7313} & \multirow{4}{*}{0.7080} & \multirow{4}{*}{0.7299} \\
\hline & 0.7 & 0.7 & 1.6 & 1.0 & 0.6727 & & & \\
\hline & 0.8 & 1.0 & 1.8 & 0.8 & 0.7037 & & & \\
\hline & 0.9 & 1.2 & 1.6 & 0.8 & 0.7252 & & & \\
\hline \multirow{3}{*}{0.6} & 0.7 & 0.5 & 1.9 & 1.0 & 0.7286 & \multirow{3}{*}{0.8384} & \multirow{3}{*}{0.8168} & \multirow{3}{*}{0.8758} \\
\hline & 0.8 & 0.6 & 1.7 & 1.0 & 0.7690 & & & \\
\hline & 0.9 & 0.6 & 1.6 & 1.0 & 0.8062 & & & \\
\hline \multirow{2}{*}{0.7} & 0.8 & 0.5 & 1.9 & 1.0 & 0.8177 & \multirow{2}{*}{0.9022} & 08992 & 10218 \\
\hline & 0.9 & 0.5 & 1.8 & 1.0 & 0.8609 & & 0.8992 & 1.0218 \\
\hline 0.8 & 0.9 & 0.5 & 1.9 & 1.0 & 0.9047 & 0.9500 & 0.9500 & 1.1678 \\
\hline
\end{tabular}




\subsubsection{Expected Costs for the 4-Subregion Strategy}

For strategy $\mathcal{S}_{4}^{\boldsymbol{\delta}}$, when $r_{1} \leq r_{2}$, the expected warranty servicing cost $E\left[C^{\Omega}\left(\psi_{4}\right)\right]$ is given by

$$
E\left[C_{A}^{\Omega}\left(\psi_{4}\right)\right]=\int_{0}^{r_{1}} E\left[C_{r}^{(1)}\left(\psi_{4}\right)\right] d G(r)+\int_{r_{1}}^{r_{2}} E\left[C_{r}^{(2)}\left(\psi_{4}\right)\right] d G(r)+\int_{r_{2}}^{\infty} E\left[C_{r}^{(3)}\left(\psi_{4}\right)\right] d G(r)
$$

where

$$
\begin{aligned}
E\left[C_{r}^{(1)}\left(\psi_{4}\right)\right]= & c_{\min } \Lambda\left(K_{1} \mid r\right) \\
+ & c_{\min }\left[\Lambda(K \mid r)-\Lambda\left(K_{3} \mid r\right)\right] e^{-\left[\Lambda\left(K_{3} \mid r\right)-\Lambda\left(K_{1} \mid r\right)\right]} \\
+ & \int_{K_{1}}^{K_{2}}\left\{\left(c_{i m p}+c_{\min }\left\{\Lambda\left[A_{1}\left(K_{2}\right) \mid r\right]-\Lambda\left[A_{1}\left(u_{1}\right) \mid r\right]\right\}\right.\right. \\
& \left.+c_{\min }\left\{\Lambda\left[A_{1}(K) \mid r\right]-\Lambda\left[A_{1}\left(K_{3}\right) \mid r\right]\right\} e^{-\left\{\Lambda\left[A_{1}\left(K_{3}\right) \mid r\right]-\Lambda\left[A_{1}\left(K_{2}\right) \mid r\right]\right\}}\right) \\
& \left.\quad \times f_{T_{K_{1} \mid r}}\left(u_{1}\right)\right\} d u_{1} \\
+ & \int_{K_{2}}^{K_{3}}\left\{\left(c_{i m p}+c_{\min }\left\{\Lambda\left[A_{1}\left(K_{3}\right) \mid r\right]-\Lambda\left[A_{1}\left(u_{1}\right) \mid r\right]\right\}\right.\right. \\
& \left.\left.+c_{\min }\left\{\Lambda\left[A_{1}(K) \mid r\right]-\Lambda\left[A_{1}\left(K_{3}\right) \mid r\right]\right\}\right) f_{T_{K_{1} \mid r}}\left(u_{1}\right)\right\} d u_{1} \\
+ & \int_{K_{2}}^{K_{3}} \int_{K_{1}}\left\{\left(c_{i m p}+c_{\min }\left\{\Lambda\left[A_{2}\left(K_{3}\right) \mid r\right]-\Lambda\left[A_{2}\left(u_{2}\right) \mid r\right]\right\}\right.\right. \\
& \left.\quad+c_{\min }\left\{\Lambda\left[A_{2}(K) \mid r\right]-\Lambda\left[A_{2}\left(K_{3}\right) \mid r\right]\right\}\right) \\
& \left.\quad \times \quad \lambda\left[A_{1}\left(u_{2}\right) \mid r\right] e^{-\left\{\Lambda\left[A_{1}\left(u_{2}\right) \mid r\right]-\Lambda\left[A_{1}\left(K_{2}\right) \mid r\right]\right\}} f_{T_{K_{1} \mid r}}\left(u_{1}\right)\right\} d u_{1} d u_{2} \\
= & \zeta\left(K_{1}, K_{2}, K_{3}, K\right)
\end{aligned}
$$

is the cost when $r \leq r_{1} \leq r_{2}$,

$$
E\left[C_{r}^{(2)}\left(\psi_{4}\right)\right]=\zeta\left(\tau_{1}, \tau_{2}, \tau_{3}, K\right)
$$

is the cost when $r_{1} \leq r \leq r_{2}$, and

$$
E\left[C_{r}^{(3)}\left(\psi_{4}\right)\right]=\zeta\left(\tau_{1}, \tau_{2}, \tau_{3}, \tau\right)
$$


is the cost when $r_{1} \leq r_{2} \leq r$. When $r_{2} \leq r_{1}$, the expected warranty servicing cost $E\left[C^{\Omega}\left(\psi_{4}\right)\right]$ is given by $E\left[C_{B}^{\Omega}\left(\psi_{4}\right)\right]=\int_{0}^{r_{2}} E\left[C_{r}^{(1)}\left(\psi_{4}\right)\right] d G(r)+\int_{r_{2}}^{r_{1}} E\left[C_{r}^{(2)}\left(\psi_{4}\right)\right] d G(r)+\int_{r_{1}}^{\infty} E\left[C_{r}^{(3)}\left(\psi_{4}\right)\right] d G(r)$ where

$$
E\left[C_{r}^{(1)}\left(\psi_{4}\right)\right]=\zeta\left(K_{1}, K_{2}, K_{3}, K\right)
$$

is the cost when $r \leq r_{2} \leq r_{1}$,

$$
E\left[C_{r}^{(2)}\left(\psi_{4}\right)\right]=\zeta\left(K_{1}, K_{2}, K_{3}, \tau\right)
$$

is the cost when $r_{2} \leq r \leq r_{1}$, and

$$
E\left[C_{r}^{(3)}\left(\psi_{4}\right)\right]=\zeta\left(\tau_{1}, \tau_{2}, \tau_{3}, \tau\right)
$$

is the cost when $r_{2} \leq r_{1} \leq r$; refer to Chapter 4 for details.

Table 6.5, Table 6.6 and Table 6.7, provide the minimum expected total warranty servicing cost $E\left[C^{\Omega}\left(\psi_{4}^{*}\right)\right]$, with the optimal partition

$$
\psi_{4}^{*}=\left(K_{1}^{*}, K_{2}^{*}, K_{3}^{*}, r_{1}^{*}\right)
$$

for different values of $\mu$ and $\delta$, for the light, medium and heavy usage categories respectively. The structure of the tables is as follows: the ratio $\mu$ of the cost of a minimal repair to the cost of a perfect repair is displayed in the first column, the ratio $\delta$ of the cost of an imperfect repair to the cost of a perfect repair is displayed in the second column, the optimal decision variables $K_{1}^{*}, K_{2}^{*}, K_{3}^{*}$ and $r_{1}^{*}$ are given in the next four columns, the corresponding expected cost $E\left[C^{\Omega}\left(\psi_{4}^{*}\right)\right]$ is given in the seventh column, the expected cost $E\left[C^{\Omega}\left(\phi_{4}^{*}\right)\right]$ for the 4-subregion restricted repair-replacement strategy by Chukova et al. [13] is provided in the eighth column, and the expected cost $E\left[C_{M R}^{\Omega}\left(\gamma^{*}\right)\right]$ for an all minimal repair strategy is presented in the last column. The expected cost $E\left[C^{\Omega}\left(\psi_{4}^{*}\right)\right]$ corresponding to the imperfect repair strategy is printed in boldface if it is the minimum for a given value of $\mu$. The tables for $\mathcal{S}_{4}^{\boldsymbol{\delta}}$ show similar results as those for $\mathcal{S}_{3}^{\boldsymbol{\delta}}$. 
For the light usage category (Table 6.5), when $\mu=0.1$, the strategy that costs the least is an all minimal repair strategy; when $\mu=0.2$, there are two imperfect repair strategies that cost less than the corresponding alternate strategies; when $\mu>0.2$, the imperfect repair strategies all yield lower costs than the other strategies, with the strategy corresponding to the lowest degree of repair $\delta, \mu<\delta<1.0$, being the best for a given value of the ratio $\mu$.

Similarly, for the medium usage category (Table 6.6), for $\mu=0.1$, the strategy that costs the least is the all minimal repair warranty strategy and for $\mu=0.2$, two of the imperfect repair strategies cost lower than the corresponding repair-replacement strategy and the all minimal repair strategy. And finally, for $0.2<\mu \leq 0.8$, the imperfect repair strategies cost lower than the corresponding alternate strategies.

For the heavy usage category (Table 6.7), for $\mu=0.1,0.2$, the strategy that costs the least is the all minimal repair strategy and for $\mu=0.3,0.4$, at least one imperfect repair strategy costs lower than the corresponding repair-replacement strategy and the all minimal repair strategy. For $\mu>0.4$, the imperfect repair strategies cost lower than the corresponding alternate repair strategies, and the imperfect repair strategy with the lowest degree of repair $\delta, \mu<\delta<1.0$, yields the lowest cost among them. 
Table 6.5: Age reduction $\mathcal{S}_{4}^{\delta}$ : costs for light usage

\begin{tabular}{|c|c|c|c|c|c|c|c|c|}
\hline$\mu$ & $\delta$ & $\mathbf{K}_{1}^{*}$ & $\mathbf{K}_{2}^{*}$ & $\mathbf{K}_{3}^{*}$ & $\mathrm{r}_{1}^{*}$ & $\mathbf{E}\left[\mathbf{C}^{\boldsymbol{\Omega}}\left(\psi_{4}^{*}\right)\right]$ & $\mathbf{E}\left[\mathbf{C}^{\boldsymbol{\Omega}}\left(\phi_{4}^{*}\right)\right]$ & $\mathbf{E}\left[\mathbf{C}_{\mathbf{M R}}^{\boldsymbol{\Omega}}\left(\gamma^{*}\right)\right]$ \\
\hline \multirow{8}{*}{0.1} & 0.2 & 0.1 & 0.2 & 0.3 & 0.2 & 0.3218 & \multirow{8}{*}{0.3367} & \multirow{8}{*}{0.3200} \\
\hline & 0.3 & 0.1 & 0.2 & 0.3 & 0.2 & 0.3236 & & \\
\hline & 0.4 & 0.1 & 0.2 & 0.3 & 0.2 & 0.3255 & & \\
\hline & 0.5 & 0.1 & 0.2 & 0.3 & 0.2 & 0.3274 & & \\
\hline & 0.6 & 0.1 & 0.2 & 0.3 & 0.2 & 0.3292 & & \\
\hline & 0.7 & 0.1 & 0.2 & 0.3 & 0.2 & 0.3311 & & \\
\hline & 0.8 & 0.1 & 0.2 & 0.3 & 0.2 & 0.3330 & & \\
\hline & 0.9 & 0.1 & 0.2 & 0.3 & 0.2 & 0.3349 & & \\
\hline \multirow{7}{*}{0.2} & 0.3 & 0.7 & 1.0 & 1.7 & 1.0 & 0.5893 & \multirow{7}{*}{0.6540} & \multirow{7}{*}{0.6400} \\
\hline & 0.4 & 0.9 & 1.0 & 1.4 & 1.0 & 0.6319 & & \\
\hline & 0.5 & 0.1 & 0.2 & 0.3 & 0.2 & 0.6450 & & \\
\hline & 0.6 & 0.1 & 0.2 & 0.3 & 0.2 & 0.6468 & & \\
\hline & 0.7 & 0.1 & 0.2 & 0.3 & 0.2 & 0.6486 & & \\
\hline & 0.8 & 0.1 & 0.2 & 0.3 & 0.2 & 0.6504 & & \\
\hline & 0.9 & 0.1 & 0.2 & 0.3 & 0.2 & 0.6522 & & \\
\hline \multirow{6}{*}{0.3} & 0.4 & 0.5 & 1.1 & 1.8 & 1.0 & 0.7682 & \multirow{6}{*}{0.9712} & \multirow{6}{*}{0.9600} \\
\hline & 0.5 & 0.7 & 0.8 & 1.7 & 1.0 & 0.8226 & & \\
\hline & 0.6 & 0.7 & 0.8 & 1.6 & 1.0 & 0.8638 & & \\
\hline & 0.7 & 0.7 & 0.8 & 1.5 & 1.0 & 0.9037 & & \\
\hline & 0.8 & 0.8 & 0.9 & 1.3 & 1.0 & 0.9383 & & \\
\hline & 0.9 & 0.9 & 1.0 & 1.1 & 1.0 & 0.9599 & & \\
\hline \multirow{5}{*}{0.4} & 0.5 & 0.4 & 1.1 & 1.9 & 1.0 & 0.9137 & \multirow{5}{*}{1.1474} & \multirow{5}{*}{1.2800} \\
\hline & 0.6 & 0.5 & 0.9 & 1.8 & 1.0 & 0.9698 & & \\
\hline & 0.7 & 0.6 & 0.7 & 1.8 & 1.0 & 1.0135 & & \\
\hline & 0.8 & 0.6 & 0.7 & 1.7 & 1.0 & 1.0556 & & \\
\hline & 0.9 & 0.6 & 0.7 & 1.6 & 1.0 & 1.1007 & & \\
\hline \multirow{4}{*}{0.5} & 0.6 & 0.3 & 1.1 & 1.9 & 1.0 & 1.0377 & \multirow{4}{*}{1.2318} & \multirow{4}{*}{1.6000} \\
\hline & 0.7 & 0.4 & 0.9 & 1.9 & 1.0 & 1.0932 & & \\
\hline & 0.8 & 0.6 & 0.7 & 1.8 & 1.0 & 1.1400 & & \\
\hline & 0.9 & 0.6 & 0.7 & 1.8 & 1.0 & 1.1835 & & \\
\hline \multirow{3}{*}{0.6} & 0.7 & 0.3 & 1.1 & 1.9 & 1.0 & 1.1464 & \multirow{3}{*}{1.2982} & \multirow{3}{*}{1.9200} \\
\hline & 0.8 & 0.4 & 1.0 & 1.9 & 1.0 & 1.2005 & & \\
\hline & 0.9 & 0.5 & 0.8 & 1.8 & 1.0 & 1.2520 & & \\
\hline \multirow{2}{*}{0.7} & 0.8 & 0.3 & 1.1 & 1.9 & 1.0 & 1.2452 & \multirow{2}{*}{1.3552} & \multirow{2}{*}{2.2400} \\
\hline & 0.9 & 0.3 & 0.9 & 1.9 & 1.0 & 1.2996 & & \\
\hline 0.8 & 0.9 & 0.3 & 1.0 & 1.9 & 1.0 & 1.3383 & 1.3952 & 2.5600 \\
\hline
\end{tabular}


Table 6.6: Age reduction $\mathcal{S}_{4}^{\boldsymbol{\delta}}$ : costs for medium usage

\begin{tabular}{|c|c|c|c|c|c|c|c|c|}
\hline$\mu$ & $\delta$ & $\mathbf{K}_{1}^{*}$ & $\mathbf{K}_{2}^{*}$ & $\mathbf{K}_{3}^{*}$ & $\mathbf{r}_{1}^{*}$ & $\mathbf{E}\left[\mathbf{C}^{\boldsymbol{\Omega}}\left(\psi_{4}^{*}\right)\right]$ & $\mathbf{E}\left[\mathbf{C}^{\boldsymbol{\Omega}}\left(\phi_{\mathbf{4}}^{*}\right)\right]$ & $\mathbf{E}\left[\mathbf{C}_{\mathbf{M R}}^{\Omega}\left(\gamma^{*}\right)\right]$ \\
\hline \multirow{8}{*}{0.1} & 0.2 & 0.5 & 0.6 & 0.7 & 0.2 & 0.3649 & \multirow{8}{*}{0.3744} & \multirow{8}{*}{0.3637} \\
\hline & 0.3 & 0.3 & 0.4 & 0.5 & 0.2 & 0.3661 & & \\
\hline & 0.4 & 0.2 & 0.3 & 0.4 & 0.2 & 0.3673 & & \\
\hline & 0.5 & 0.2 & 0.3 & 0.4 & 0.2 & 0.3685 & & \\
\hline & 0.6 & 0.2 & 0.3 & 0.4 & 0.2 & 0.3697 & & \\
\hline & 0.7 & 0.2 & 0.3 & 0.4 & 0.2 & 0.3709 & & \\
\hline & 0.8 & 0.2 & 0.3 & 0.4 & 0.2 & 0.3721 & & \\
\hline & 0.9 & 0.2 & 0.3 & 0.4 & 0.2 & 0.3732 & & \\
\hline \multirow{7}{*}{0.2} & 0.3 & 0.6 & 1.1 & 1.7 & 1.0 & 0.6574 & \multirow{7}{*}{0.7363} & \multirow{7}{*}{0.7274} \\
\hline & 0.4 & 0.8 & 0.9 & 1.5 & 1.0 & 0.7046 & & \\
\hline & 0.5 & 1.4 & 1.5 & 1.6 & 0.6 & 0.7290 & & \\
\hline & 0.6 & 1.3 & 1.4 & 1.5 & 0.2 & 0.7316 & & \\
\hline & 0.7 & 0.8 & 0.9 & 1.0 & 0.2 & 0.7328 & & \\
\hline & 0.8 & 0.7 & 0.8 & 0.9 & 0.2 & 0.7340 & & \\
\hline & 0.9 & 0.6 & 0.7 & 0.8 & 0.2 & 0.7352 & & \\
\hline \multirow{6}{*}{0.3} & 0.4 & 0.5 & 1.2 & 1.8 & 1.0 & 0.8553 & \multirow{6}{*}{1.0911} & \multirow{6}{*}{1.0911} \\
\hline & 0.5 & 0.6 & 0.9 & 1.7 & 1.0 & 0.9129 & & \\
\hline & 0.6 & 0.7 & 0.8 & 1.6 & 1.0 & 0.9539 & & \\
\hline & 0.7 & 0.7 & 0.8 & 1.5 & 1.0 & 0.9953 & & \\
\hline & 0.8 & 0.7 & 0.8 & 1.4 & 1.0 & 1.0356 & & \\
\hline & 0.9 & 0.9 & 1.0 & 1.4 & 0.8 & 1.0701 & & \\
\hline \multirow{5}{*}{0.4} & 0.5 & 0.4 & 1.1 & 1.9 & 1.0 & 1.0165 & \multirow{5}{*}{1.2536} & \multirow{5}{*}{1.4549} \\
\hline & 0.6 & 0.5 & 1.0 & 1.8 & 1.0 & 1.0736 & & \\
\hline & 0.7 & 0.6 & 0.7 & 1.8 & 1.0 & 1.1183 & & \\
\hline & 0.8 & 0.6 & 0.7 & 1.7 & 1.0 & 1.1587 & & \\
\hline & 0.9 & 0.6 & 0.7 & 1.6 & 1.0 & 1.2044 & & \\
\hline \multirow{4}{*}{0.5} & 0.6 & 0.4 & 1.1 & 1.9 & 1.0 & 1.1541 & \multirow{4}{*}{1.3465} & \multirow{4}{*}{1.8186} \\
\hline & 0.7 & 0.4 & 1.0 & 1.9 & 1.0 & 1.2096 & & \\
\hline & 0.8 & 0.5 & 0.8 & 1.8 & 1.0 & 1.2582 & & \\
\hline & 0.9 & 0.6 & 0.7 & 1.8 & 1.0 & 1.2987 & & \\
\hline \multirow{3}{*}{0.6} & 0.7 & 0.3 & 1.1 & 1.9 & 1.0 & 1.2747 & \multirow{3}{*}{1.4268} & \multirow{3}{*}{2.1823} \\
\hline & 0.8 & 0.4 & 1.0 & 1.9 & 1.0 & 1.3287 & & \\
\hline & 0.9 & 0.5 & 0.8 & 1.9 & 1.0 & 1.3814 & & \\
\hline \multirow{2}{*}{0.7} & 0.8 & 0.3 & 1.1 & 1.9 & 1.0 & 1.3843 & \multirow{2}{*}{1.5049} & \multirow{2}{*}{2.5460} \\
\hline & 0.9 & 0.4 & 1.0 & 1.9 & 1.0 & 1.4392 & & \\
\hline 0.8 & 0.9 & 0.3 & 1.0 & 1.9 & 1.0 & 1.4874 & 1.5493 & 2.9097 \\
\hline
\end{tabular}


Table 6.7: Age reduction $\mathcal{S}_{4}^{\boldsymbol{\delta}}$ : costs for heavy usage

\begin{tabular}{|c|c|c|c|c|c|c|c|c|}
\hline$\mu$ & $\delta$ & $\mathbf{K}_{1}^{*}$ & $\mathbf{K}_{2}^{*}$ & $\mathbf{K}_{3}^{*}$ & $\mathbf{r}_{1}^{*}$ & $\mathbf{E}\left[\mathbf{C}^{\boldsymbol{\Omega}}\left(\psi_{4}^{*}\right)\right]$ & $\mathbf{E}\left[\mathbf{C}^{\boldsymbol{\Omega}}\left(\phi_{\mathbf{4}}^{*}\right)\right]$ & $\mathbf{E}\left[\mathbf{C}_{\mathbf{M R}}^{\Omega}\left(\gamma^{*}\right)\right]$ \\
\hline \multirow{8}{*}{0.1} & 0.2 & 0.4 & 0.5 & 0.6 & 0.2 & 0.1470 & \multirow{8}{*}{0.1550} & \multirow{8}{*}{0.1460} \\
\hline & 0.3 & 0.3 & 0.4 & 0.5 & 0.2 & 0.1480 & & \\
\hline & 0.4 & 0.3 & 0.4 & 0.5 & 0.2 & 0.1490 & & \\
\hline & 0.5 & 0.2 & 0.3 & 0.4 & 0.2 & 0.1500 & & \\
\hline & 0.6 & 0.2 & 0.3 & 0.4 & 0.2 & 0.1510 & & \\
\hline & 0.7 & 0.2 & 0.3 & 0.4 & 0.2 & 0.1520 & & \\
\hline & 0.8 & 0.2 & 0.3 & 0.4 & 0.2 & 0.1530 & & \\
\hline & 0.9 & 0.2 & 0.3 & 0.4 & 0.2 & 0.1540 & & \\
\hline \multirow{7}{*}{0.2} & 0.3 & 1.7 & 1.8 & 1.9 & 0.2 & 0.2928 & \multirow{7}{*}{0.2998} & \multirow{7}{*}{0.2919} \\
\hline & 0.4 & 1.2 & 1.3 & 1.4 & 0.2 & 0.2938 & & \\
\hline & 0.5 & 0.9 & 1.0 & 1.1 & 0.2 & 0.2949 & & \\
\hline & 0.6 & 0.7 & 0.8 & 0.9 & 0.2 & 0.2959 & & \\
\hline & 0.7 & 0.7 & 0.8 & 0.9 & 0.2 & 0.2969 & & \\
\hline & 0.8 & 0.6 & 0.7 & 0.8 & 0.2 & 0.2979 & & \\
\hline & 0.9 & 0.6 & 0.7 & 0.8 & 0.2 & 0.2989 & & \\
\hline \multirow{6}{*}{0.3} & 0.4 & 1.0 & 1.1 & 1.9 & 0.8 & 0.4252 & \multirow{6}{*}{0.4445} & \multirow{6}{*}{0.4379} \\
\hline & 0.5 & 1.7 & 1.8 & 1.9 & 0.2 & 0.4394 & & \\
\hline & 0.6 & 1.7 & 1.8 & 1.9 & 0.2 & 0.4404 & & \\
\hline & 0.7 & 1.7 & 1.8 & 1.9 & 0.2 & 0.4415 & & \\
\hline & 0.8 & 1.4 & 1.5 & 1.6 & 0.2 & 0.4425 & & \\
\hline & 0.9 & 1.2 & 1.3 & 1.4 & 0.2 & 0.4435 & & \\
\hline \multirow{5}{*}{0.4} & 0.5 & 0.6 & 1.0 & 1.7 & 1.0 & 0.5332 & \multirow{5}{*}{0.5888} & \multirow{5}{*}{0.5839} \\
\hline & 0.6 & 1.0 & 1.1 & 1.8 & 0.8 & 0.5680 & & \\
\hline & 0.7 & 1.7 & 1.8 & 1.9 & 0.6 & 0.5841 & & \\
\hline & 0.8 & 1.7 & 1.8 & 1.9 & 0.2 & 0.5868 & & \\
\hline & 0.9 & 1.7 & 1.8 & 1.9 & 0.2 & 0.5878 & & \\
\hline \multirow{4}{*}{0.5} & 0.6 & 0.4 & 1.0 & 1.8 & 1.0 & 0.6306 & \multirow{4}{*}{0.7331} & \multirow{4}{*}{0.7299} \\
\hline & 0.7 & 0.6 & 0.7 & 1.6 & 1.0 & 0.6734 & & \\
\hline & 0.8 & 1.0 & 1.1 & 1.8 & 0.8 & 0.7051 & & \\
\hline & 0.9 & 1.2 & 1.3 & 1.5 & 0.8 & 0.7262 & & \\
\hline \multirow{3}{*}{0.6} & 0.7 & 0.4 & 1.0 & 1.8 & 1.0 & 0.7223 & \multirow{3}{*}{0.8404} & \multirow{3}{*}{0.8758} \\
\hline & 0.8 & 0.6 & 0.7 & 1.7 & 1.0 & 0.7693 & & \\
\hline & 0.9 & 0.6 & 0.7 & 1.6 & 1.0 & 0.8078 & & \\
\hline \multirow{2}{*}{0.7} & 0.8 & 0.3 & 0.9 & 1.9 & 1.0 & 0.8095 & \multirow{2}{*}{0.8919} & \multirow{2}{*}{1.0218} \\
\hline & 0.9 & 0.5 & 0.6 & 1.8 & 1.0 & 0.8605 & & \\
\hline 0.8 & 0.9 & 0.3 & 0.9 & 1.9 & 1.0 & 0.8947 & 0.9148 & 1.1678 \\
\hline
\end{tabular}




\subsection{Results for Intensity Reduction Model}

The expected total warranty servicing cost $E\left[C^{\Omega}\left(\psi_{n}\right)\right]$, for the intensity reduction model is given by equation (5.14) (on page 102) when $r_{1} \leq r_{2}$ and equation (5.15) (on page 106) when $r_{2} \leq r_{1}$.

\subsubsection{Expected Costs for the 3-Subregion Strategy}

For strategy $\mathcal{S}_{3}^{\boldsymbol{\delta}}$, when $r_{1} \leq r_{2}$, the expected warranty servicing cost $E\left[C^{\Omega}\left(\psi_{3}\right)\right]$ is given by

$$
E\left[C_{A}^{\Omega}\left(\psi_{3}\right)\right]=\int_{0}^{r_{1}} E\left[C_{r}^{(1)}\left(\psi_{3}\right)\right] d G(r)+\int_{r_{1}}^{r_{2}} E\left[C_{r}^{(2)}\left(\psi_{3}\right)\right] d G(r)+\int_{r_{2}}^{\infty} E\left[C_{r}^{(3)}\left(\psi_{3}\right)\right] d G(r)
$$

where

$$
\begin{aligned}
E\left[C_{r}^{(1)}\left(\psi_{3}\right)\right]= & c_{\min } \Lambda\left(K_{1} \mid r\right) \\
+ & c_{\min }\left[\Lambda(K \mid r)-\Lambda\left(K_{2} \mid r\right)\right] e^{-\left[\Lambda\left(K_{2} \mid r\right)-\Lambda\left(K_{1} \mid r\right)\right]} \\
+ & \int_{K_{1}}^{K_{2}}\left\{\left(c_{i m p}+c_{\min } \Lambda_{1}\left(K_{2} \mid r\right)+c_{\min }\left\{\Lambda_{1}(K \mid r)-\Lambda_{1}\left(K_{2} \mid r\right)\right\}\right)\right. \\
& \left.\quad \times f_{T_{K_{1} \mid r}}\left(u_{1}\right)\right\} d u_{1} \\
= & \xi\left(K_{1}, K_{2}, K\right)
\end{aligned}
$$

is the cost when $r \leq r_{1} \leq r_{2}$,

$$
E\left[C_{r}^{(2)}\left(\psi_{3}\right)\right]=\xi\left(\tau_{1}, \tau_{2}, K\right)
$$

is the cost when $r_{1} \leq r \leq r_{2}$, and

$$
E\left[C_{r}^{(3)}\left(\psi_{3}\right)\right]=\xi\left(\tau_{1}, \tau_{2}, \tau\right)
$$

is the cost when $r_{1} \leq r_{2} \leq r$. When $r_{2} \leq r_{1}$, the expected warranty servicing cost $E\left[C^{\Omega}\left(\psi_{3}\right)\right]$ is given by

$$
E\left[C_{B}^{\Omega}\left(\psi_{3}\right)\right]=\int_{0}^{r_{2}} E\left[C_{r}^{(1)}\left(\psi_{3}\right)\right] d G(r)+\int_{r_{2}}^{r_{1}} E\left[C_{r}^{(2)}\left(\psi_{3}\right)\right] d G(r)+\int_{r_{1}}^{\infty} E\left[C_{r}^{(3)}\left(\psi_{3}\right)\right] d G(r)
$$


where

$$
E\left[C_{r}^{(1)}\left(\psi_{3}\right)\right]=\xi\left(K_{1}, K_{2}, K\right)
$$

is the cost when $r \leq r_{2} \leq r_{1}$,

$$
E\left[C_{r}^{(2)}\left(\psi_{3}\right)\right]=\xi\left(K_{1}, K_{2}, \tau\right)
$$

is the cost when $r_{2} \leq r \leq r_{1}$, and

$$
E\left[C_{r}^{(3)}\left(\psi_{3}\right)\right]=\xi\left(\tau_{1}, \tau_{2}, \tau\right)
$$

is the cost when $r_{2} \leq r_{1} \leq r$; refer to Chapter 5 for more information.

Table 6.8, Table 6.9 and Table 6.10 provide the minimum expected total warranty servicing cost $E\left[C^{\Omega}\left(\psi_{3}^{*}\right)\right]$, with the optimal partition

$$
\psi_{3}^{*}=\left(K_{1}^{*}, K_{2}^{*}, r_{1}^{*}\right)
$$

for different values of $\mu$ and $\delta$, for the light, medium and heavy usage categories, respectively. The structure of the tables is as follows: the ratio $\mu$ of the cost of a minimal repair to the cost of a perfect repair is displayed in the first column, the ratio $\delta$ of the cost of an imperfect repair to the cost of a perfect repair is displayed in the second column, the optimal decision variables $K_{1}^{*}, K_{2}^{*}$ and $r_{1}^{*}$ are given in the next three columns, the corresponding expected cost $E\left[C^{\Omega}\left(\psi_{3}^{*}\right)\right]$ is given in the sixth column, the expected cost $E\left[C^{\Omega}\left(\hat{\phi}_{3}{ }^{*}\right)\right]$ for the 3-subregion restricted repair-replacement strategy by Iskandar et al. [21] is displayed in the seventh column, and the expected cost $E\left[C_{M R}^{\Omega}\left(\gamma^{*}\right)\right]$ for an all minimal repair strategy is presented in the last column. The expected cost $E\left[C^{\Omega}\left(\psi_{3}^{*}\right)\right]$ corresponding to the imperfect repair strategy is printed in boldface if it is the minimum for a given value of $\mu$.

For the light usage category (Table 6.8), when $\mu=0.1$, the strategy that costs the least is an all minimal repair strategy; when $\mu=0.2$, the imperfect repair strategy with $\delta=0.3$ costs the least when compared to corresponding strategies; when $0.2<\mu \leq 0.5$, all expect one of the imperfect repair strategies yield lower costs than the corresponding alternate 
strategies, with the strategy corresponding to the lowest degree of repair $\delta, \mu<\delta<1.0$, being the best for a given value of $\mu$. For $\mu>0.5$, as the degree of repair $\delta$ increases the cost decreases, and hence, having replacements in place of imperfect repairs is more feasible.

For the medium usage category (Table 6.9), as in the light category, for $\mu=0.1$, the strategy that costs the least is the all minimal repair strategy and for $\mu=0.2$, the imperfect repair strategy with $\delta=0.3$ costs lower than the corresponding repair-replacement strategy and the all minimal repair strategy. For $\mu=0.3,0.4$, the imperfect repair strategies all cost lower than the corresponding alternate strategies. For $\mu>0.4$, as the degree of repair $\delta$ increases the cost decreases, and therefore, the corresponding repair-replacement strategy by Iskandar et al. [21] costs the least.

For the heavy usage category (Table 6.10), for $\mu=0.1,0.2$, the strategy that costs the least is the all minimal repair strategy and for $\mu=0.3,0.4$, two of the imperfect repair strategies cost lower than the corresponding repair-replacement strategy and the all minimal repair strategy. For $\mu>$ 0.4 , the imperfect repair strategies cost lower than the corresponding alternate strategies, with the strategy corresponding to the smallest degree of repair $\delta, \mu<\delta<1.0$, being the best among them. 
Table 6.8: Intensity reduction $\mathcal{S}_{3}^{\boldsymbol{\delta}}$ : costs for light usage

\begin{tabular}{|c|c|c|c|c|c|c|c|}
\hline$\mu$ & $\delta$ & $\mathbf{K}_{1}^{*}$ & $\mathbf{K}_{\mathbf{2}}^{*}$ & $\mathrm{r}_{1}^{*}$ & $\mathbf{E}\left[\mathbf{C}^{\boldsymbol{\Omega}}\left(\psi_{\mathbf{3}}^{*}\right)\right]$ & $\mathbf{E}\left[\mathbf{C}^{\boldsymbol{\Omega}}\left(\phi_{\mathbf{3}}^{*}\right)\right]$ & $\mathbf{E}\left[\mathbf{C}_{\mathbf{M R}}^{\Omega}\left(\gamma^{*}\right)\right]$ \\
\hline \multirow{8}{*}{0.1} & 0.2 & 0.1 & 0.2 & 0.2 & 0.3209 & \multirow{8}{*}{0.3281} & \multirow{8}{*}{0.3200} \\
\hline & 0.3 & 0.1 & 0.2 & 0.2 & 0.3218 & & \\
\hline & 0.4 & 0.1 & 0.2 & 0.2 & 0.3227 & & \\
\hline & 0.5 & 0.1 & 0.2 & 0.2 & 0.3236 & & \\
\hline & 0.6 & 0.1 & 0.2 & 0.2 & 0.3245 & & \\
\hline & 0.7 & 0.1 & 0.2 & 0.2 & 0.3254 & & \\
\hline & 0.8 & 0.1 & 0.2 & 0.2 & 0.3263 & & \\
\hline & 0.9 & 0.1 & 0.2 & 0.2 & 0.3272 & & \\
\hline \multirow{7}{*}{0.2} & 0.3 & 0.7 & 1.5 & 1.0 & 0.6260 & \multirow{7}{*}{0.6469} & \multirow{7}{*}{0.6400} \\
\hline & 0.4 & 0.2 & 0.3 & 0.2 & 0.6416 & & \\
\hline & 0.5 & 0.1 & 0.2 & 0.2 & 0.6425 & & \\
\hline & 0.6 & 0.1 & 0.2 & 0.2 & 0.6434 & & \\
\hline & 0.7 & 0.1 & 0.2 & 0.2 & 0.6443 & & \\
\hline & 0.8 & 0.1 & 0.2 & 0.2 & 0.6451 & & \\
\hline & 0.9 & 0.1 & 0.2 & 0.2 & 0.6460 & & \\
\hline \multirow{6}{*}{0.3} & 0.4 & 0.6 & 1.8 & 1.0 & 0.8531 & \multirow{6}{*}{0.9656} & \multirow{6}{*}{0.9600} \\
\hline & 0.5 & 0.6 & 1.6 & 1.0 & 0.8885 & & \\
\hline & 0.6 & 0.7 & 1.5 & 1.0 & 0.9180 & & \\
\hline & 0.7 & 0.8 & 1.3 & 1.0 & 0.9410 & & \\
\hline & 0.8 & 0.9 & 1.2 & 1.0 & 0.9569 & & \\
\hline & 0.9 & 0.9 & 1.0 & 1.0 & 0.9623 & & \\
\hline \multirow{5}{*}{0.4} & 0.5 & 0.6 & 1.9 & 1.0 & 1.0425 & \multirow{5}{*}{1.1401} & \multirow{5}{*}{1.2800} \\
\hline & 0.6 & 0.6 & 1.8 & 1.0 & 1.0661 & & \\
\hline & 0.7 & 0.6 & 1.7 & 1.0 & 1.0872 & & \\
\hline & 0.8 & 0.6 & 1.6 & 1.0 & 1.1065 & & \\
\hline & 0.9 & 0.6 & 1.6 & 1.0 & 1.1239 & & \\
\hline \multirow{4}{*}{0.5} & 0.6 & 0.6 & 1.9 & 1.0 & 1.1978 & \multirow{4}{*}{1.2258} & \multirow{4}{*}{1.6000} \\
\hline & 0.7 & 0.6 & 1.8 & 1.0 & 1.2072 & & \\
\hline & 0.8 & 0.6 & 1.8 & 1.0 & 1.2141 & & \\
\hline & 0.9 & 0.6 & 1.8 & 1.0 & 1.2211 & & \\
\hline \multirow{3}{*}{0.6} & 0.7 & 0.6 & 1.9 & 1.0 & 1.3202 & \multirow{3}{*}{1.2971} & \multirow{3}{*}{1.9200} \\
\hline & 0.8 & 0.6 & 1.9 & 1.0 & 1.3132 & & \\
\hline & 0.9 & 0.6 & 1.9 & 1.0 & 1.3062 & & \\
\hline \multirow{2}{*}{0.7} & 0.8 & 0.5 & 1.9 & 1.0 & 1.4097 & \multirow{2}{*}{1.3627} & \multirow{2}{*}{2.2400} \\
\hline & 0.9 & 0.6 & 1.9 & 1.0 & 1.3862 & & \\
\hline 0.8 & 0.9 & 0.5 & 1.9 & 1.0 & 1.4661 & 1.4263 & 2.5600 \\
\hline
\end{tabular}


Table 6.9: Intensity reduction $\mathcal{S}_{3}^{\delta}$ : costs for medium usage

\begin{tabular}{|c|c|c|c|c|c|c|c|}
\hline$\mu$ & $\delta$ & $\mathbf{K}_{1}^{*}$ & $\mathbf{K}_{2}^{*}$ & $\mathbf{r}_{1}^{*}$ & $\mathbf{E}\left[\mathbf{C}^{\boldsymbol{\Omega}}\left(\psi_{3}^{*}\right)\right]$ & $\mathbf{E}\left[\mathbf{C}^{\boldsymbol{\Omega}}\left(\phi_{3}^{*}\right)\right]$ & $\mathbf{E}\left[\mathbf{C}_{\mathbf{M R}}^{\Omega}\left(\gamma^{*}\right)\right.$ \\
\hline \multirow{8}{*}{0.1} & 0.2 & 0.5 & 0.6 & 0.2 & 0.3643 & \multirow{8}{*}{0.3691} & \multirow{8}{*}{0.3637} \\
\hline & 0.3 & 0.3 & 0.4 & 0.2 & 0.3649 & & \\
\hline & 0.4 & 0.3 & 0.4 & 0.2 & 0.3655 & & \\
\hline & 0.5 & 0.3 & 0.4 & 0.2 & 0.3661 & & \\
\hline & 0.6 & 0.2 & 0.3 & 0.2 & 0.3667 & & \\
\hline & 0.7 & 0.2 & 0.3 & 0.2 & 0.3673 & & \\
\hline & 0.8 & 0.2 & 0.3 & 0.2 & 0.3679 & & \\
\hline & 0.9 & 0.2 & 0.3 & 0.2 & 0.3685 & & \\
\hline \multirow{7}{*}{0.2} & 0.3 & 0.7 & 1.5 & 1.0 & 0.7033 & \multirow{7}{*}{0.7319} & \multirow{7}{*}{0.7274} \\
\hline & 0.4 & 1.8 & 1.9 & 0.4 & 0.7281 & & \\
\hline & 0.5 & 1.5 & 1.6 & 0.2 & 0.7289 & & \\
\hline & 0.6 & 0.9 & 1.0 & 0.2 & 0.7296 & & \\
\hline & 0.7 & 0.8 & 0.9 & 0.2 & 0.7301 & & \\
\hline & 0.8 & 0.7 & 0.8 & 0.2 & 0.7307 & & \\
\hline & 0.9 & 0.7 & 0.8 & 0.2 & 0.7313 & & \\
\hline \multirow{6}{*}{0.3} & 0.4 & 0.6 & 1.8 & 1.0 & 0.9579 & \multirow{6}{*}{1.0894} & \multirow{6}{*}{1.0911} \\
\hline & 0.5 & 0.6 & 1.6 & 1.0 & 0.9908 & & \\
\hline & 0.6 & 0.7 & 1.5 & 1.0 & 1.0189 & & \\
\hline & 0.7 & 0.7 & 1.4 & 1.0 & 1.0431 & & \\
\hline & 0.8 & 0.8 & 1.3 & 1.0 & 1.0635 & & \\
\hline & 0.9 & 0.9 & 1.4 & 0.8 & 1.0791 & & \\
\hline \multirow{5}{*}{0.4} & 0.5 & 0.6 & 1.9 & 1.0 & 1.1713 & \multirow{5}{*}{1.2420} & \multirow{5}{*}{1.4549} \\
\hline & 0.6 & 0.6 & 1.8 & 1.0 & 1.1884 & & \\
\hline & 0.7 & 0.6 & 1.7 & 1.0 & 1.2038 & & \\
\hline & 0.8 & 0.6 & 1.7 & 1.0 & 1.2178 & & \\
\hline & 0.9 & 0.6 & 1.6 & 1.0 & 1.2304 & & \\
\hline \multirow{4}{*}{0.5} & 0.6 & 0.6 & 1.9 & 1.0 & 1.3463 & \multirow{4}{*}{1.3390} & \multirow{4}{*}{1.8186} \\
\hline & 0.7 & 0.6 & 1.9 & 1.0 & 1.3459 & & \\
\hline & 0.8 & 0.6 & 1.8 & 1.0 & 1.3440 & & \\
\hline & 0.9 & 0.6 & 1.8 & 1.0 & 1.3417 & & \\
\hline \multirow{3}{*}{0.6} & 0.7 & 0.6 & 1.9 & 1.0 & 1.4834 & \multirow{3}{*}{1.4249} & \multirow{3}{*}{2.1823} \\
\hline & 0.8 & 0.6 & 1.9 & 1.0 & 1.4642 & & \\
\hline & 0.9 & 0.6 & 1.9 & 1.0 & 1.4449 & & \\
\hline \multirow{2}{*}{0.7} & 0.8 & 0.6 & 1.9 & 1.0 & 1.5829 & \multirow{2}{*}{1.5066} & \multirow{2}{*}{2.5460} \\
\hline & 0.9 & 0.6 & 1.9 & 1.0 & 1.5447 & & \\
\hline 0.8 & 0.9 & 0.6 & 1.9 & 1.0 & 1.6445 & 1.5875 & 2.9097 \\
\hline
\end{tabular}


Table 6.10: Intensity reduction $\mathcal{S}_{3}^{\delta}$ : costs for heavy usage

\begin{tabular}{|c|c|c|c|c|c|c|c|}
\hline$\mu$ & $\delta$ & $\mathbf{K}_{1}^{*}$ & $\mathbf{K}_{\mathbf{2}}^{*}$ & $r_{1}^{*}$ & $\mathbf{E}\left[\mathbf{C}^{\boldsymbol{\Omega}}\left(\psi_{3}^{*}\right)\right]$ & $\mathbf{E}\left[\mathbf{C}^{\boldsymbol{\Omega}}\left(\phi_{\mathbf{3}}^{*}\right)\right]$ & $\mathbf{E}\left[\mathbf{C}_{\mathbf{M R}}^{\Omega}\left(\gamma^{*}\right)\right]$ \\
\hline \multirow{8}{*}{0.1} & 0.2 & 0.5 & 0.6 & 0.2 & 0.1465 & \multirow{8}{*}{0.1505} & \multirow{8}{*}{0.1460} \\
\hline & 0.3 & 0.3 & 0.4 & 0.2 & 0.1470 & & \\
\hline & 0.4 & 0.3 & 0.4 & 0.2 & 0.1475 & & \\
\hline & 0.5 & 0.3 & 0.4 & 0.2 & 0.1480 & & \\
\hline & 0.6 & 0.3 & 0.4 & 0.2 & 0.1485 & & \\
\hline & 0.7 & 0.2 & 0.3 & 0.2 & 0.1490 & & \\
\hline & 0.8 & 0.2 & 0.3 & 0.2 & 0.1495 & & \\
\hline & 0.9 & 0.2 & 0.3 & 0.2 & 0.1500 & & \\
\hline \multirow{7}{*}{0.2} & 0.3 & 1.8 & 1.9 & 0.2 & 0.2924 & \multirow{7}{*}{0.2959} & \multirow{7}{*}{0.2919} \\
\hline & 0.4 & 1.0 & 1.1 & 0.2 & 0.2929 & & \\
\hline & 0.5 & 0.8 & 0.9 & 0.2 & 0.2934 & & \\
\hline & 0.6 & 0.7 & 0.8 & 0.2 & 0.2939 & & \\
\hline & 0.7 & 0.7 & 0.8 & 0.2 & 0.2944 & & \\
\hline & 0.8 & 0.6 & 0.7 & 0.2 & 0.2949 & & \\
\hline & 0.9 & 0.6 & 0.7 & 0.2 & 0.2954 & & \\
\hline \multirow{6}{*}{0.3} & 0.4 & 1.1 & 1.6 & 0.8 & 0.4365 & \multirow{6}{*}{0.4412} & \multirow{6}{*}{0.4379} \\
\hline & 0.5 & 1.8 & 1.9 & 0.2 & 0.4387 & & \\
\hline & 0.6 & 1.8 & 1.9 & 0.2 & 0.4392 & & \\
\hline & 0.7 & 1.6 & 1.7 & 0.2 & 0.4397 & & \\
\hline & 0.8 & 1.4 & 1.5 & 0.2 & 0.4402 & & \\
\hline & 0.9 & 1.2 & 1.3 & 0.2 & 0.4407 & & \\
\hline \multirow{5}{*}{0.4} & 0.5 & 0.6 & 1.6 & 1.0 & 0.5569 & \multirow{5}{*}{0.5864} & \multirow{5}{*}{0.5839} \\
\hline & 0.6 & 1.1 & 1.6 & 0.8 & 0.5811 & & \\
\hline & 0.7 & 1.8 & 1.9 & 0.2 & 0.5849 & & \\
\hline & 0.8 & 1.8 & 1.9 & 0.2 & 0.5854 & & \\
\hline & 0.9 & 1.8 & 1.9 & 0.2 & 0.5859 & & \\
\hline \multirow{4}{*}{0.5} & 0.6 & 0.5 & 1.7 & 1.0 & 0.6630 & \multirow{4}{*}{0.7313} & \multirow{4}{*}{0.7299} \\
\hline & 0.7 & 0.8 & 1.9 & 0.8 & 0.6943 & & \\
\hline & 0.8 & 1.0 & 1.7 & 0.8 & 0.7164 & & \\
\hline & 0.9 & 1.6 & 1.9 & 0.6 & 0.7286 & & \\
\hline \multirow{3}{*}{0.6} & 0.7 & 0.5 & 1.8 & 1.0 & 0.7585 & \multirow{3}{*}{0.8384} & \multirow{3}{*}{0.8758} \\
\hline & 0.8 & 0.6 & 1.7 & 1.0 & 0.7908 & & \\
\hline & 0.9 & 0.8 & 1.9 & 0.8 & 0.8172 & & \\
\hline \multirow{2}{*}{0.7} & 0.8 & 0.5 & 1.9 & 1.0 & 0.8451 & \multirow{2}{*}{0.9022} & \multirow{2}{*}{1.0218} \\
\hline & 0.9 & 0.5 & 1.8 & 1.0 & 0.8757 & & \\
\hline 0.8 & 0.9 & 0.4 & 1.9 & 1.0 & 0.9220 & 0.9500 & 1.1678 \\
\hline
\end{tabular}




\subsubsection{Expected Costs for the 4-Subregion Strategy}

For strategy $\mathcal{S}_{4}^{\boldsymbol{\delta}}$, when $r_{1} \leq r_{2}$, the expected warranty servicing cost $E\left[C^{\Omega}\left(\psi_{4}\right)\right]$ is given by

$E\left[C_{A}^{\Omega}\left(\psi_{4}\right)\right]=\int_{0}^{r_{1}} E\left[C_{r}^{(1)}\left(\psi_{4}\right)\right] d G(r)+\int_{r_{1}}^{r_{2}} E\left[C_{r}^{(2)}\left(\psi_{4}\right)\right] d G(r)+\int_{r_{2}}^{\infty} E\left[C_{r}^{(3)}\left(\psi_{4}\right)\right] d G(r)$

where

$$
\begin{aligned}
& E\left[C_{r}^{(1)}\left(\psi_{4}\right)\right]=c_{\min } \Lambda\left(K_{1} \mid r\right) \\
& +c_{\min }\left[\Lambda(K \mid r)-\Lambda\left(K_{3} \mid r\right)\right] e^{-\left[\Lambda\left(K_{3} \mid r\right)-\Lambda\left(K_{1} \mid r\right)\right]} \\
& +\int_{K_{1}}^{K_{2}}\left\{\left(c_{i m p}+c_{\text {min }} \Lambda_{1}\left(K_{2} \mid r\right)+c_{\min }\left\{\Lambda_{1}(K \mid r)-\Lambda_{1}\left(K_{3} \mid r\right)\right\}\right.\right. \\
& \left.\left.\times e^{-\left\{\Lambda_{1}\left(K_{3} \mid r\right)-\Lambda_{1}\left(K_{2} \mid r\right)\right\}}\right) f_{T_{K_{1} \mid r}}\left(u_{1}\right)\right\} d u_{1} \\
& +\int_{K_{2}}^{K_{3}}\left\{\left(c_{i m p}+c_{\min } \Lambda_{1}\left(K_{3} \mid r\right)+c_{\min }\left\{\Lambda_{1}(K \mid r)-\Lambda_{1}\left(K_{3} \mid r\right)\right\}\right)\right. \\
& \left.\times f_{T_{K_{1} \mid r}}\left(u_{1}\right)\right\} d u_{1} \\
& +\int_{K_{2}}^{K_{3}} \int_{K_{1}}^{K_{2}}\left\{\left(c_{i m p}+c_{\min } \Lambda_{2}\left(K_{3} \mid r\right)+c_{\min }\left\{\Lambda_{2}(K \mid r)-\Lambda_{2}\left(K_{3} \mid r\right)\right\}\right.\right. \\
& \left.\times e^{-\left\{\Lambda_{2}\left(K_{3} \mid r\right)-\Lambda_{2}\left(K_{3} \mid r\right)\right\}}\right) \\
& \left.\times \lambda_{1}\left(u_{2} \mid r\right) e^{-\left\{\Lambda_{1}\left(u_{2} \mid r\right)-\Lambda_{1}\left(K_{2} \mid r\right)\right\}} f_{T_{K_{1} \mid r}}\left(u_{1}\right)\right\} d u_{1} d u_{2} \\
& =\xi\left(K_{1}, K_{2}, K_{3}, K\right)
\end{aligned}
$$

is the cost when $r \leq r_{1} \leq r_{2}$,

$$
E\left[C_{r}^{(2)}\left(\psi_{4}\right)\right]=\xi\left(\tau_{1}, \tau_{2}, \tau_{3}, K\right)
$$

is the cost when $r_{1} \leq r \leq r_{2}$, and

$$
E\left[C_{r}^{(3)}\left(\psi_{4}\right)\right]=\xi\left(\tau_{1}, \tau_{2}, \tau_{3}, \tau\right)
$$

is the cost when $r_{1} \leq r_{2} \leq r$. When $r_{2} \leq r_{1}$, the expected warranty servicing cost $E\left[C^{\Omega}\left(\psi_{4}\right)\right]$ is given by

$$
E\left[C_{B}^{\Omega}\left(\psi_{4}\right)\right]=\int_{0}^{r_{2}} E\left[C_{r}^{(1)}\left(\psi_{4}\right)\right] d G(r)+\int_{r_{2}}^{r_{1}} E\left[C_{r}^{(2)}\left(\psi_{4}\right)\right] d G(r)+\int_{r_{1}}^{\infty} E\left[C_{r}^{(3)}\left(\psi_{4}\right)\right] d G(r)
$$


where

$$
E\left[C_{r}^{(1)}\left(\psi_{4}\right)\right]=\xi\left(K_{1}, K_{2}, K_{3}, K\right)
$$

is the cost when $r \leq r_{2} \leq r_{1}$,

$$
E\left[C_{r}^{(2)}\left(\psi_{4}\right)\right]=\xi\left(K_{1}, K_{2}, K_{3}, \tau\right)
$$

is the cost when $r_{2} \leq r \leq r_{1}$, and

$$
E\left[C_{r}^{(3)}\left(\psi_{4}\right)\right]=\xi\left(\tau_{1}, \tau_{2}, \tau_{3}, \tau\right)
$$

is the cost when $r_{2} \leq r_{1} \leq r$; see Chapter 5 for information on deriving the expected cost for the intensity reduction model.

Table 6.11, Table 6.12 and Table 6.13 provide the minimum expected total warranty servicing cost $E\left[C^{\Omega}\left(\psi_{4}^{*}\right)\right]$, with the optimal partition

$$
\psi_{4}^{*}=\left(K_{1}^{*}, K_{2}^{*}, K_{4}^{*}, r_{1}^{*}\right)
$$

for different values of $\mu$ and $\delta$, for the light, medium and heavy usage categories, respectively. The structure of the tables is as follows; the ratio $\mu$ of the cost of a minimal repair to the cost of a perfect repair is displayed in the first column, the ratio $\delta$ of the cost of an imperfect repair to the cost of a perfect repair is displayed in the second column, the optimal decision variables $K_{1}^{*}, K_{2}^{*}, K_{3}^{*}$ and $r_{1}^{*}$ are given in the next four columns, the corresponding expected cost $E\left[C^{\Omega}\left(\psi_{4}^{*}\right)\right]$ is given in the seventh column, the expected cost $E\left[C^{\Omega}\left(\phi_{4}^{*}\right)\right]$ for the 4-subregion restricted repair-replacement strategy by Chukova et al [13], where all repairs in the first and last subregions are minimal, the first repair in each of the intermediate subregions is perfect (replacement) and all subsequent repairs in the subregion are minimal, is displayed in the eighth column, and the expected cost $E\left[C_{M R}^{\Omega}\left(\gamma^{*}\right)\right]$ for an all minimal repair strategy is presented in the last column. The expected cost $E\left[C^{\Omega}\left(\psi_{4}^{*}\right)\right]$ corresponding to the imperfect repair strategy is printed in boldface if it is the minimum for a given value of $\mu$.

For the light usage category (Table 6.11), when $\mu=0.12$, the strategy that costs the least is an all minimal repair strategy; when $\mu=0.2$, the 
imperfect repair strategy with $\delta=0.3$ costs less than the other strategies; when $0.2<\mu \leq 0.7$, all except one of the imperfect repair strategies yield lower costs than the corresponding alternate strategies, with the strategy corresponding to the lowest degree of repair $\delta, \mu<\delta<1.0$, being the best for a given value of $\mu$. For $\mu>0.7$, having replacements in place of imperfect repairs costs the least.

For the medium usage category (Table 6.12), as in the light category, for $\mu=0.1$, the strategy that costs the least is the all minimal repair strategy and for $\mu=0.2$, the imperfect repair strategy with $\delta=0.3$ costs lower than the corresponding repair-replacement strategy and the all minimal repair strategy. For $0.2<\mu \leq 0.7$, all except one of the imperfect repair strategies cost lower than the corresponding alternate strategies. For $\mu>$ 0.7, the corresponding repair-replacement strategy by Chukova et al. [13] costs lower than the imperfect repair strategy and the all minimal repair strategy.

For the heavy usage category (Table 6.13), for $\mu=0.1,0.2$, the strategy that costs the least is the all minimal repair strategy and for $\mu=0.3,0.4$, at least one imperfect repair strategy costs lower than the corresponding repair-replacement strategy by Chukova et al. [13] and the all minimal repair strategy. For $0.4<\mu \leq 0.8$, the imperfect repair strategies cost lower than the corresponding alternate strategies, with the strategy with the smallest $\delta, \mu<\delta<1.0$, yielding the lowest cost among them. 
Table 6.11: Intensity reduction $\mathcal{S}_{4}^{\boldsymbol{\delta}}$ : costs for light usage

\begin{tabular}{|c|c|c|c|c|c|c|c|c|}
\hline$\mu$ & $\delta$ & $\mathbf{K}_{1}^{*}$ & $\mathbf{K}_{2}^{*}$ & $\mathbf{K}_{3}^{*}$ & $\mathbf{r}_{1}^{*}$ & $\mathbf{E}\left[\mathbf{C}^{\boldsymbol{\Omega}}\left(\psi_{4}^{*}\right)\right]$ & $\mathbf{E}\left[\mathbf{C}^{\boldsymbol{\Omega}}\left(\phi_{4}^{*}\right)\right]$ & $\mathbf{E}\left[\mathbf{C}_{\mathbf{M R}}^{\boldsymbol{\Omega}}\left(\gamma^{*}\right)\right]$ \\
\hline \multirow{8}{*}{0.1} & 0.2 & 0.1 & 0.2 & 0.3 & 0.2 & 0.3218 & \multirow{8}{*}{0.3367} & \multirow{8}{*}{0.3200} \\
\hline & 0.3 & 0.1 & 0.2 & 0.3 & 0.2 & 0.3237 & & \\
\hline & 0.4 & 0.1 & 0.2 & 0.3 & 0.2 & 0.3255 & & \\
\hline & 0.5 & 0.1 & 0.2 & 0.3 & 0.2 & 0.3274 & & \\
\hline & 0.6 & 0.1 & 0.2 & 0.3 & 0.2 & 0.3293 & & \\
\hline & 0.7 & 0.1 & 0.2 & 0.3 & 0.2 & 0.3311 & & \\
\hline & 0.8 & 0.1 & 0.2 & 0.3 & 0.2 & 0.3330 & & \\
\hline & 0.9 & 0.1 & 0.2 & 0.3 & 0.2 & 0.3349 & & \\
\hline \multirow{7}{*}{0.2} & 0.3 & 0.7 & 0.8 & 1.4 & 1.0 & 0.6262 & \multirow{7}{*}{0.6540} & \multirow{7}{*}{0.6400} \\
\hline & 0.4 & 0.1 & 0.2 & 0.3 & 0.2 & 0.6433 & & \\
\hline & 0.5 & 0.1 & 0.2 & 0.3 & 0.2 & 0.6450 & & \\
\hline & 0.6 & 0.1 & 0.2 & 0.3 & 0.2 & 0.6468 & & \\
\hline & 0.7 & 0.1 & 0.2 & 0.3 & 0.2 & 0.6486 & & \\
\hline & 0.8 & 0.1 & 0.2 & 0.3 & 0.2 & 0.6504 & & \\
\hline & 0.9 & 0.1 & 0.2 & 0.3 & 0.2 & 0.6522 & & \\
\hline \multirow{6}{*}{0.3} & 0.4 & 0.4 & 1.1 & 1.7 & 1.0 & 0.8411 & \multirow{6}{*}{0.9712} & \multirow{6}{*}{0.9600} \\
\hline & 0.5 & 0.6 & 0.7 & 1.6 & 1.0 & 0.8889 & & \\
\hline & 0.6 & 0.6 & 0.7 & 1.4 & 1.0 & 0.9211 & & \\
\hline & 0.7 & 0.7 & 0.8 & 1.3 & 1.0 & 0.9454 & & \\
\hline & 0.8 & 0.9 & 1.0 & 1.1 & 1.0 & 0.9588 & & \\
\hline & 0.9 & 0.8 & 0.9 & 1.0 & 1.0 & 0.9666 & & \\
\hline \multirow{5}{*}{0.4} & 0.5 & 0.4 & 1.1 & 1.8 & 1.0 & 1.0119 & \multirow{5}{*}{1.1474} & \multirow{5}{*}{1.2800} \\
\hline & 0.6 & 0.4 & 0.9 & 1.7 & 1.0 & 1.0592 & & \\
\hline & 0.7 & 0.6 & 0.7 & 1.7 & 1.0 & 1.0885 & & \\
\hline & 0.8 & 0.6 & 0.7 & 1.6 & 1.0 & 1.1106 & & \\
\hline & 0.9 & 0.6 & 0.7 & 1.6 & 1.0 & 1.1308 & & \\
\hline \multirow{4}{*}{0.5} & 0.6 & 0.3 & 1.1 & 1.9 & 1.0 & 1.1496 & \multirow{4}{*}{1.2318} & \multirow{4}{*}{1.6000} \\
\hline & 0.7 & 0.4 & 1.0 & 1.8 & 1.0 & 1.1881 & & \\
\hline & 0.8 & 0.5 & 0.7 & 1.8 & 1.0 & 1.2124 & & \\
\hline & 0.9 & 0.5 & 0.6 & 1.7 & 1.0 & 1.2237 & & \\
\hline \multirow{3}{*}{0.6} & 0.7 & 0.3 & 1.1 & 1.9 & 1.0 & 1.2593 & \multirow{3}{*}{1.2982} & \multirow{3}{*}{1.9200} \\
\hline & 0.8 & 0.3 & 1.0 & 1.9 & 1.0 & 1.2867 & & \\
\hline & 0.9 & 0.4 & 0.8 & 1.8 & 1.0 & 1.3001 & & \\
\hline \multirow{2}{*}{0.7} & 0.8 & 0.3 & 1.1 & 1.9 & 1.0 & 1.3425 & \multirow{2}{*}{1.3552} & \multirow{2}{*}{2.2400} \\
\hline & 0.9 & 0.3 & 0.9 & 1.9 & 1.0 & 1.3551 & & \\
\hline 0.8 & 0.9 & 0.3 & 1.1 & 1.9 & 1.0 & 1.3999 & 1.3952 & 2.5600 \\
\hline
\end{tabular}


Table 6.12: Intensity reduction $\mathcal{S}_{4}^{\boldsymbol{\delta}}$ : costs for medium usage

\begin{tabular}{|c|c|c|c|c|c|c|c|c|}
\hline$\mu$ & $\delta$ & $\mathbf{K}_{1}^{*}$ & $\mathbf{K}_{2}^{*}$ & $\mathbf{K}_{3}^{*}$ & $\mathbf{r}_{1}^{*}$ & $\mathbf{E}\left[\mathbf{C}^{\boldsymbol{\Omega}}\left(\psi_{4}^{*}\right)\right]$ & $\mathbf{E}\left[\mathbf{C}^{\boldsymbol{\Omega}}\left(\phi_{4}^{*}\right)\right]$ & $\mathbf{E}\left[\mathbf{C}_{\mathbf{M R}}^{\Omega}\left(\gamma^{*}\right)\right]$ \\
\hline \multirow{8}{*}{0.1} & 0.2 & 0.4 & 0.5 & 0.6 & 0.2 & 0.3649 & \multirow{8}{*}{0.3744} & \multirow{8}{*}{0.3637} \\
\hline & 0.3 & 0.3 & 0.4 & 0.5 & 0.2 & 0.3661 & & \\
\hline & 0.4 & 0.2 & 0.3 & 0.4 & 0.2 & 0.3673 & & \\
\hline & 0.5 & 0.2 & 0.3 & 0.4 & 0.2 & 0.3685 & & \\
\hline & 0.6 & 0.2 & 0.3 & 0.4 & 0.2 & 0.3697 & & \\
\hline & 0.7 & 0.2 & 0.3 & 0.4 & 0.2 & 0.3709 & & \\
\hline & 0.8 & 0.2 & 0.3 & 0.4 & 0.2 & 0.3721 & & \\
\hline & 0.9 & 0.2 & 0.3 & 0.4 & 0.2 & 0.3732 & & \\
\hline \multirow{7}{*}{0.2} & 0.3 & 0.6 & 0.8 & 1.5 & 1.0 & 0.7032 & \multirow{7}{*}{0.7363} & \multirow{7}{*}{0.7274} \\
\hline & 0.4 & 1.7 & 1.8 & 1.9 & 0.2 & 0.7290 & & \\
\hline & 0.5 & 1.2 & 1.3 & 1.4 & 0.2 & 0.7304 & & \\
\hline & 0.6 & 0.9 & 1.0 & 1.1 & 0.2 & 0.7317 & & \\
\hline & 0.7 & 0.7 & 0.8 & 0.9 & 0.2 & 0.7328 & & \\
\hline & 0.8 & 0.7 & 0.8 & 0.9 & 0.2 & 0.7340 & & \\
\hline & 0.9 & 0.6 & 0.7 & 0.8 & 0.2 & 0.7352 & & \\
\hline \multirow{6}{*}{0.3} & 0.4 & 0.4 & 1.1 & 1.8 & 1.0 & 0.9390 & \multirow{6}{*}{1.0911} & \multirow{6}{*}{1.0911} \\
\hline & 0.5 & 0.6 & 0.7 & 1.6 & 1.0 & 0.9904 & & \\
\hline & 0.6 & 0.6 & 0.7 & 1.5 & 1.0 & 1.0226 & & \\
\hline & 0.7 & 0.7 & 0.8 & 1.4 & 1.0 & 1.0498 & & \\
\hline & 0.8 & 0.8 & 0.9 & 1.4 & 0.8 & 1.0706 & & \\
\hline & 0.9 & 1.0 & 1.1 & 1.3 & 0.8 & 1.0843 & & \\
\hline \multirow{5}{*}{0.4} & 0.5 & 0.4 & 1.1 & 1.9 & 1.0 & 1.1279 & \multirow{5}{*}{1.2536} & \multirow{5}{*}{1.4549} \\
\hline & 0.6 & 0.4 & 1.0 & 1.7 & 1.0 & 1.1753 & & \\
\hline & 0.7 & 0.6 & 0.7 & 1.7 & 1.0 & 1.2043 & & \\
\hline & 0.8 & 0.6 & 0.7 & 1.7 & 1.0 & 1.2231 & & \\
\hline & 0.9 & 0.6 & 0.7 & 1.6 & 1.0 & 1.2388 & & \\
\hline \multirow{4}{*}{0.5} & 0.6 & 0.3 & 1.1 & 1.9 & 1.0 & 1.2800 & \multirow{4}{*}{1.3465} & \multirow{4}{*}{1.8186} \\
\hline & 0.7 & 0.4 & 1.0 & 1.8 & 1.0 & 1.3163 & & \\
\hline & 0.8 & 0.5 & 0.8 & 1.8 & 1.0 & 1.3396 & & \\
\hline & 0.9 & 0.6 & 0.7 & 1.8 & 1.0 & 1.3453 & & \\
\hline \multirow{3}{*}{0.6} & 0.7 & 0.3 & 1.1 & 1.9 & 1.0 & 1.4006 & \multirow{3}{*}{1.4268} & \multirow{3}{*}{2.1823} \\
\hline & 0.8 & 0.3 & 1.0 & 1.9 & 1.0 & 1.4245 & & \\
\hline & 0.9 & 0.4 & 0.8 & 1.8 & 1.0 & 1.4351 & & \\
\hline \multirow{2}{*}{0.7} & 0.8 & 0.3 & 1.1 & 1.9 & 1.0 & 1.4921 & \multirow{2}{*}{1.5049} & \multirow{2}{*}{2.5460} \\
\hline & 0.9 & 0.3 & 1.0 & 1.9 & 1.0 & 1.5002 & & \\
\hline 0.8 & 0.9 & 0.3 & 1.1 & 1.9 & 1.0 & 1.5553 & 1.5493 & 2.9097 \\
\hline
\end{tabular}


Table 6.13: Intensity reduction $\mathcal{S}_{4}^{\boldsymbol{\delta}}$ : costs for heavy usage

\begin{tabular}{|c|c|c|c|c|c|c|c|c|}
\hline$\mu$ & $\delta$ & $\mathbf{K}_{1}^{*}$ & $\mathbf{K}_{2}^{*}$ & $\mathbf{K}_{3}^{*}$ & $\mathbf{r}_{1}^{*}$ & $\mathbf{E}\left[\mathbf{C}^{\boldsymbol{\Omega}}\left(\psi_{4}^{*}\right)\right]$ & $\mathbf{E}\left[\mathbf{C}^{\boldsymbol{\Omega}}\left(\phi_{\mathbf{4}}^{*}\right)\right]$ & $\mathbf{E}\left[\mathbf{C}_{\mathbf{M R}}^{\Omega}\left(\gamma^{*}\right)\right]$ \\
\hline \multirow{8}{*}{0.1} & 0.2 & 0.4 & 0.5 & 0.6 & 0.2 & 0.1470 & \multirow{8}{*}{0.1550} & \multirow{8}{*}{0.1460} \\
\hline & 0.3 & 0.3 & 0.4 & 0.5 & 0.2 & 0.1480 & & \\
\hline & 0.4 & 0.2 & 0.3 & 0.4 & 0.2 & 0.1490 & & \\
\hline & 0.5 & 0.2 & 0.3 & 0.4 & 0.2 & 0.1500 & & \\
\hline & 0.6 & 0.2 & 0.3 & 0.4 & 0.2 & 0.1510 & & \\
\hline & 0.7 & 0.2 & 0.3 & 0.4 & 0.2 & 0.1520 & & \\
\hline & 0.8 & 0.2 & 0.3 & 0.4 & 0.2 & 0.1530 & & \\
\hline & 0.9 & 0.2 & 0.3 & 0.4 & 0.2 & 0.1540 & & \\
\hline \multirow{7}{*}{0.2} & 0.3 & 1.7 & 1.8 & 1.9 & 0.2 & 0.2928 & \multirow{7}{*}{0.2998} & \multirow{7}{*}{0.2919} \\
\hline & 0.4 & 1.0 & 1.1 & 1.2 & 0.2 & 0.2939 & & \\
\hline & 0.5 & 0.8 & 0.9 & 1.0 & 0.2 & 0.2949 & & \\
\hline & 0.6 & 0.7 & 0.8 & 0.9 & 0.2 & 0.2959 & & \\
\hline & 0.7 & 0.6 & 0.7 & 0.8 & 0.2 & 0.2969 & & \\
\hline & 0.8 & 0.6 & 0.7 & 0.8 & 0.2 & 0.2979 & & \\
\hline & 0.9 & 0.6 & 0.7 & 0.8 & 0.2 & 0.2987 & & \\
\hline \multirow{6}{*}{0.3} & 0.4 & 1.1 & 1.2 & 1.5 & 0.8 & 0.4366 & \multirow{6}{*}{0.4445} & \multirow{6}{*}{0.4379} \\
\hline & 0.5 & 1.7 & 1.8 & 1.9 & 0.2 & 0.4395 & & \\
\hline & 0.6 & 1.7 & 1.8 & 1.9 & 0.2 & 0.4405 & & \\
\hline & 0.7 & 1.5 & 1.6 & 1.7 & 0.2 & 0.4415 & & \\
\hline & 0.8 & 1.3 & 1.4 & 1.5 & 0.2 & 0.4425 & & \\
\hline & 0.9 & 1.2 & 1.3 & 1.4 & 0.2 & 0.4435 & & \\
\hline \multirow{5}{*}{0.4} & 0.5 & 0.6 & 1.0 & 1.6 & 1.0 & 0.5561 & \multirow{5}{*}{0.5888} & \multirow{5}{*}{0.5839} \\
\hline & 0.6 & 1.1 & 1.2 & 1.5 & 0.8 & 0.5815 & & \\
\hline & 0.7 & 1.7 & 1.8 & 1.9 & 0.2 & 0.5859 & & \\
\hline & 0.8 & 1.7 & 1.8 & 1.9 & 0.2 & 0.5869 & & \\
\hline & 0.9 & 1.7 & 1.8 & 1.9 & 0.2 & 0.5879 & & \\
\hline \multirow{4}{*}{0.5} & 0.6 & 0.4 & 1.0 & 1.7 & 1.0 & 0.6594 & \multirow{4}{*}{0.7331} & \multirow{4}{*}{0.7299} \\
\hline & 0.7 & 0.8 & 0.9 & 1.9 & 0.8 & 0.6947 & & \\
\hline & 0.8 & 1.0 & 1.1 & 1.7 & 0.8 & 0.7176 & & \\
\hline & 0.9 & 1.7 & 1.8 & 1.9 & 0.6 & 0.7290 & & \\
\hline \multirow{3}{*}{0.6} & 0.7 & 0.3 & 1.0 & 1.8 & 1.0 & 0.7522 & \multirow{3}{*}{0.8404} & \multirow{3}{*}{0.8758} \\
\hline & 0.8 & 0.5 & 0.6 & 1.7 & 1.0 & 0.7910 & & \\
\hline & 0.9 & 0.8 & 0.9 & 1.9 & 0.8 & 0.8184 & & \\
\hline \multirow{2}{*}{0.7} & 0.8 & 0.3 & 1.0 & 1.9 & 1.0 & 0.8364 & \multirow{2}{*}{0.8919} & \multirow{2}{*}{1.0218} \\
\hline & 0.9 & 0.5 & 0.6 & 1.8 & 1.0 & 0.8755 & & \\
\hline 0.8 & 0.9 & 0.3 & 0.9 & 1.9 & 1.0 & 0.9118 & 0.9148 & 1.1678 \\
\hline
\end{tabular}




\subsection{Optimal Warranty Repair Strategy}

In this section, we summarize the results obtained and displayed in previous sections. In the numerical example, we have applied the 3 -subregion and 4-subregion imperfect repair strategies. In Chukova et al. [13], the numerical results for the 5 and 6 subregion repair-replacement strategies are presented. Even though in some cases the higher-subregion strategies cost less, the strategy comparisons here are based on the 3- and 4-subregion strategies. The all minimal repair strategy is denotes by $\mathcal{S}_{M R}$, and for $\delta \in(0,1]$, the imperfect repair strategies and the repair-replacement strategy are denoted by $\mathcal{S}_{n}^{\delta}$, where $n$ is the number of subregions, and $\delta$ is the vector of degrees of repair, which in this example is just $\delta=\delta$. For each of the imperfect repair models, presented in Table 6.14 and Table 6.15, respectively, are the optimal restricted warranty repair strategies corresponding to different costs $\mu$ of minimal repairs.

Table 6.14: Optimal warranty repair strategy - Age reduction model

\begin{tabular}{|c|c|c|c|}
\hline$\mu$ & Light usage & Medium usage & Heavy usage \\
\hline 0.1 & $\mathcal{S}_{M R}$ & $\mathcal{S}_{M R}$ & $\mathcal{S}_{M R}$ \\
0.2 & $\mathcal{S}_{4}^{0.3}$ & $\mathcal{S}_{4}^{0.3}$ & $\mathcal{S}_{M R}$ \\
0.3 & $\mathcal{S}_{4}^{0.4}$ & $\mathcal{S}_{4}^{0.4}$ & $\mathcal{S}_{3}^{0.4}$ \\
0.4 & $\mathcal{S}_{4}^{0.5}$ & $\mathcal{S}_{4}^{0.5}$ & $\mathcal{S}_{4}^{0.5}$ \\
0.5 & $\mathcal{S}_{4}^{0.6}$ & $\mathcal{S}_{4}^{0.6}$ & $\mathcal{S}_{4}^{0.6}$ \\
0.6 & $\mathcal{S}_{4}^{0.7}$ & $\mathcal{S}_{4}^{0.7}$ & $\mathcal{S}_{4}^{0.7}$ \\
0.7 & $\mathcal{S}_{4}^{0.8}$ & $\mathcal{S}_{4}^{0.8}$ & $\mathcal{S}_{4}^{0.8}$ \\
0.8 & $\mathcal{S}_{4}^{0.9}$ & $\mathcal{S}_{4}^{0.9}$ & $\mathcal{S}_{4}^{0.9}$ \\
0.9 & $\mathcal{S}_{4}^{1.0}$ & $\mathcal{S}_{4}^{1.0}$ & $\mathcal{S}_{4}^{1.0}$ \\
\hline
\end{tabular}

In the age reduction model, for the light and medium usage categories, when the cost of a minimal repair is low $\mu=0.1$, the optimal warranty repair strategy is the all minimal repair strategy, and when the cost of a minimal repair is high $\mu=0.9$, the optimal strategy is the 4 -subregion 
repair-replacement strategy. For $0.1<\mu<0.9$, the 4 -subregion imperfect repair strategy with the lowest degree of repair $\delta, \mu<\delta<1.0$, is optimal. For the heavy usage category, when $\mu$ is low, the optimal warranty repair strategy is either the all minimal repair strategy or the 3-subregion imperfect repair strategy with the lowest degree of repair $\delta$. For higher values of $\mu$, the optimal strategy is the 4-subregion imperfect repair strategy with the smallest $\delta$, and when $\mu=0.9$, the optimal warranty repair strategy for the heavy usage category is the 4-subregion repair-replacement strategy.

Table 6.15: Optimal warranty repair strategy - Intensity reduction model

\begin{tabular}{|c|c|c|c|}
\hline$\mu$ & Light usage & Medium usage & Heavy usage \\
\hline 0.1 & $\mathcal{S}_{M R}$ & $\mathcal{S}_{M R}$ & $\mathcal{S}_{M R}$ \\
0.2 & $\mathcal{S}_{3}^{0.3}$ & $\mathcal{S}_{4}^{0.3}$ & $\mathcal{S}_{M R}$ \\
0.3 & $\mathcal{S}_{4}^{0.4}$ & $\mathcal{S}_{4}^{0.4}$ & $\mathcal{S}_{3}^{0.4}$ \\
0.4 & $\mathcal{S}_{4}^{0.5}$ & $\mathcal{S}_{4}^{0.5}$ & $\mathcal{S}_{4}^{0.5}$ \\
0.5 & $\mathcal{S}_{4}^{0.6}$ & $\mathcal{S}_{4}^{0.6}$ & $\mathcal{S}_{4}^{0.6}$ \\
0.6 & $\mathcal{S}_{3}^{0.7}$ & $\mathcal{S}_{4}^{0.7}$ & $\mathcal{S}_{4}^{0.7}$ \\
0.7 & $\mathcal{S}_{4}^{0.8}$ & $\mathcal{S}_{4}^{0.8}$ & $\mathcal{S}_{4}^{0.8}$ \\
0.8 & $\mathcal{S}_{4}^{1.0}$ & $\mathcal{S}_{4}^{1.0}$ & $\mathcal{S}_{4}^{0.9}$ \\
0.9 & $\mathcal{S}_{4}^{1.0}$ & $\mathcal{S}_{4}^{1.0}$ & $\mathcal{S}_{4}^{1.0}$ \\
\hline
\end{tabular}

In the intensity reduction model, for the light and heavy usage categories, for low values of $\mu$ the optimal warranty repair strategy is either the all minimal repair strategy or the 3-subregion imperfect repair strategy; for intermediate values of $\mu$, the 4 -subregion strategies result in lower costs when compared to the 3 -subregion strategies; for high values of $\mu$, the 4 -subregion repair-replacement strategy is optimal. For the medium usage category, the optimal strategy when $\mu=0.1$ is the all minimal repair strategy; for all intermediate values of $\mu$, the optimal strategy is the 4-subregion imperfect repair strategy with the lowest corresponding $\delta$; for high values of $\mu$ the optimal strategy is the 4 -subregion repair-replacement strategy. 


\section{Chapter 7}

\section{Conclusion and Future Research}

In this thesis, we extended the warranty repair-replacement strategy proposed by Iskandar et al. [21] and Chukova et al. [13] to an imperfect repair strategy. The new strategy is a generalization of the repair-replacement strategy in terms of the degree of repairs. The imperfect repair strategy studied, is for repairable products sold under a nonrenewing twodimensional free-replacement warranty policy. The warranty region is divided into $n$ subregions; all repairs in the first and last subregion are minimal; in each of the intermediate subregions, the first repair is imperfect and all subsequent repairs are minimal. Since the strategy is restricted, these subregions are defined by $n$ decision variables whose optimal values are found by minimizing the expected warranty servicing cost over the warranty region.

In the new warranty repair strategy, repairs with different degrees $\delta$, $0<\delta<1$, are considered. For comparison reasons, we used an example proposed by Iskandar et al. [21] and extended by Chukova et al. [13] to numerically illustrate our findings for the imperfect repair strategy.

To model the imperfect repairs, we used an extension of the age reduction model purposed by Doyen and Gaudoin [16], and a modification of the intensity reduction model by Chukova et al. [11]. 
For each of the two imperfect repair models, we

- derived the density functions for the imperfect repair times in the intermediate subregions;

- derived an expression for the expected total warranty servicing costs for the $n$-subregion warranty repair strategy;

- numerically illustrated the procedure for finding the optimal partitioning of the warranty region and the optimal warranty repair strategy, using an example by Iskandar et al. [21].

For the chosen example, we presented numerical results for the 3 - and 4-subregion imperfect repair strategies, and provided comparison with previously studied repair-replacement strategies. The unrestricted strategy by Chukova and Johnston [14] did better in some cases, but since the imperfect repair strategy proposed here is restricted, the final comparison for finding the optimal repair strategy was with the restricted 3-subregion strategy by Iskandar et al. [21] and the restricted 4-subregion strategy by Chukova et al. [13]. Also included were comparisons with an all minimal repair strategy. For both the age reduction model and the intensity reduction model, the results show that in many cases performing imperfect repairs in place of replacements can significantly reduce warranty costs.

The results derived in this thesis can be extended and modified in a number of ways:

- In the expressions derived for the expected warranty servicing costs, the repair costs were assumed to be constant; in the numerical example we modified these costs to be proportional to the degree of the imperfect repair. They can be further modified into more realistic cost functions based on actual or empirical data.

- In the numerical example, we considered the 3- and 4-subregion imperfect repair strategies. It is possible that an imperfect repair strategy with more than four subregions is the optimal strategy. Finding 
the optimal number of subregions for the imperfect repair strategy could be the next step in finding the best repair strategy.

- The derived results are for a free-replacement warranty policy. Similarly, the imperfect repair strategy can be developed for pro-rata and combination warranty policies.

- The repair strategy is for a two-dimensional non-renewing warranty; it would be of interest to study a two-dimensional renewing repairreplacement warranty strategy.

- The optimization procedure used to obtain the numerical results is based on a coarse grid. The suggested strategies are optimal when optimizing over this grid. Finding an efficient optimization procedure that would produce more accurate results could be another direction to further this study. 


\section{Bibliography}

[1] Aven, T., AND Jensen, U. Stochastic models in reliability. Springer, 1999.

[2] BAIK, J., MURTHY, D., AND JACK, N. Two-dimensional failure modeling with minimal repair. Naval Research Logistics 51, 3 (2003), 345362.

[3] BARLOW, R., AND Hunter, L. Optimum preventive maintenance policies. Operations Research 8, 1 (Feb. 1960), 90-100.

[4] Barlow, R. E., Proschan, F., And Hunter, L. C. Mathematical theory of reliability. SIAM, 1996.

[5] BIEDenWeG, F. M. Warranty analysis: Consumer value vs. manufacturers cost. Unpublished PhD thesis, Stanford University, Stanford, CA, 1981.

[6] BlisChKe, W. R., AND MuRThY, D. N. P. Warranty cost analysis. CRC Press, 1994.

[7] BlischKe, W. R., AND Murthy, D. N. P. Product warranty handbook. CRC Press, Nov. 1995.

[8] Blischke, W. R., AND Murthy, D. N. P. Reliability. Wiley-IEEE, 2000. 
[9] Block, H. W., Borges, W. S., And Savits, T. H. Age-Dependent minimal repair. Journal of Applied Probability 22, 2 (June 1985), 370-385.

[10] BROWN, M., AND PROSCHAN, F. Imperfect repair. Journal of Applied Probability 20, 4 (Dec. 1983), 851-859.

[11] Chukova, S., ARnOld, R., AND WANG, D. Q. Warranty analysis: An approach to modeling imperfect repairs. International Journal of Production Economics 89, 1 (May 2004), 57-68.

[12] Chukova, S., HAYAKAWA, Y., AND ARnOLD, R. Warranty analysis: Estimation of the degree of imperfect repair via a bayesian approach. In Recent Advances in Stochastic Operations Research. Canada, 2005.

[13] Chukova, S., Hayakawa, Y., And Johnston, M. R. Optimal twodimensional warranty repair strategy. Proceedings of the Institution of Mechanical Engineers 221, Number 4 (Dec. 2007), 265-273.

[14] Chukova, S., AND Johnston, M. Two-dimensional warranty repair strategy based on minimal and complete repairs. Mathematical and Computer Modelling 44, 11-12 (Dec. 2006), 1133-1143.

[15] CHUN, Y. H., AND TANG, K. Cost analysis of two-attribute warranty policies based on the product usage rate. Engineering Management, IEEE Transactions on 46, 2 (1999), 201-209.

[16] Doyen, L., AND GAudoin, O. Classes of imperfect repair models based on reduction of failure intensity or virtual age. Reliability Engineering \& System Safety 84, 1 (Apr. 2004), 45-56.

[17] Grimmett, G., AND StiRZAKer, D. Probability and random processes. Oxford University Press, 2001.

[18] HOKSTAD, P. The failure intensity process and the formulation of reliability and maintenance models. Reliability Engineering $\mathcal{E}$ System Safety 58, 1 (Oct. 1997), 69-82. 
[19] Hollander, M., Presnell, B., And Sethuraman, J. Nonparametric methods for imperfect repair models. The Annals of Statistics 20, 2 (June 1992), 879-896.

[20] IsKandar, B. P., AND Murthy, D. N. P. Repair-replace strategies for two-dimensional warranty policies. Mathematical and computer modelling 38 (2003), 1233-1241.

[21] Iskandar, B. P., Murthy, D. N. P., AND JACK, N. A new repairreplace strategy for items sold with a two-dimensional warranty. Computers \& Operations Research 32, 3 (Mar. 2005), 669-682.

[22] Iskandar, B. P., Wilson, R. J., And Murthy, D. N. P. Twodimensional combination warranty policies. RAIRO. Operations Research 28, n0 1 (1994), 57-75.

[23] JACK, N. Age-reduction models for imperfect maintenance. IMA J Management Math 9, 4 (Oct. 1998), 347-354.

[24] JACK, N., AND DER DUYN SCHOUTEN, F. V. Optimal repair-replace strategies for a warranted product. International Journal of Production Economics 67, 1 (Aug. 2000), 95-100.

[25] JACK, N., AND MURTHY, D. N. P. A servicing strategy for items sold under warranty. The Journal of the Operational Research Society 52, 11 (Nov. 2001), 1284-1288.

[26] JaQuette, D. L. Approximations to the renewal function $m(t)$. Rand, 1970.

[27] JARdine, A. K. S., AND TsAnG, A. H. C. Maintenance, replacement, and reliability. CRC Press, 2006.

[28] Johnson, N. L., AND KotZ, S. Continuous univariate distributions I and II. Wiley, 1970. 
[29] KiJIMA, M. Some results for repairable systems with general repair. Journal of Applied Probability 26, 1 (Mar. 1989), 89-102.

[30] KIM, H. G., AND RAO, B. M. Expected warranty cost of two-attribute free-replacement warranties based on a bivariate exponential distribution. Computers \& Industrial Engineering 38, 4 (Dec. 2000), 425-434.

[31] Langseth, H., AND Lindqvist, B. H. A maintenance model for components exposed to several failure mechanisms and imperfect repair. Mathematical and Statistical Methods in Reliability. Series on Quality, Reliability and Engineering Statistics 7 (2002), 415-430.

[32] LaWless, J., HU, J., AND CAO, J. Methods for the estimation of failure distributions and rates from automobile warranty data. Lifetime Data Analysis 1, 3 (1995), 227-240.

[33] Lawless, J. F., And Thiagarajah, K. A point-process model incorporating renewals and time trends, with application to repairable systems. Technometrics 38, 2 (1996), 131-138.

[34] McGuire, E. P. Industrial product warranties: Policies and practices. Tech. rep., New York, 1980.

[35] MetTAs, A., AND ZHAO, W. Modeling and analysis of repairable systems with general repair. In Reliability and Maintainability Symposium, 2005. Proceedings. Annual (2005), pp. 176-182.

[36] Moskowitz, H., AND ChUN, Y. H. A poisson regression model for two-attribute warranty policies. Naval Research Logistics 41, 3 (1994), 355-376.

[37] Murthy, D. Product warranty and reliability. Annals of Operations Research 143, 1 (Mar. 2006), 133-146. 
[38] Murthy, D., Baik, J., Wilson, R., and Bulmer, M. TwoDimensional failure modeling. In Springer Handbook of Engineering Statistics. 2006, pp. 97-111.

[39] Murthy, D. N. P., Iskandar, B. P., And Wilson, R. J. TwoDimensional Failure-Free warranty policies: Two-Dimensional point process models. Operations Research 43, 2 (Apr. 1995), 356-366.

[40] Nakagawa, T. Imperfect Preventive-Maintenance. Reliability, IEEE Transactions on $R-28,5$ (1979), 402.

[41] NGUYEN, D. G. Studies in warranty policies and product reliability. Unpublished PhD thesis, The University of Queensland, Australia, 1994.

[42] NGuyen, D. G., AND Murthy, D. N. P. An optimal policy for servicing warranty. The Journal of the Operational Research Society 37, 11 (Nov. 1986), 1081-1088.

[43] Nguyen, D. G., And Murthy, D. N. P. Optimal replace-repair strategy for servicing products sold with warranty. European Journal of Operational Research 39, 2 (Mar. 1989), 206-212.

[44] PARK, K. S. Optimal number of minimal repairs before replacement. Reliability, IEEE Transactions on R-28, 2 (1979), 137-140.

[45] PHAm, H. Handbook of reliability engineering. Birkhuser, 2003.

[46] Pham, H., AND WANG, H. Imperfect maintenance. European Journal of Operational Research 94, 3 (Nov. 1996), 425-438.

[47] Phelps, R. I. Replacement policies under minimal repair. The Journal of the Operational Research Society 32, 7 (July 1981), 549-554.

[48] Ross, S. M. Stochastic Processes. John Wiley \& Sons, Incorporated, Feb. 1983.

[49] Ross, S. M. Introduction to probability models. Academic Press, 2007. 
[50] SHEU, S., AND GRIFFITH, W. S. Multivariate imperfect repair. Journal of Applied Probability 29, 4 (Dec. 1992), 947-956.

[51] XIE, M. On the solution of renewal-type integral equations. Communications in Statistics - Simulation and Computation 18, 1 (1989), 281.

[52] YANG, S., KobZA, J. E., AND NACHLAS, J. A. Bivariate failure modeling. In Reliability and Maintainability Symposium, 2000. Proceedi gs. Annual (2000), pp. 281-287.

[53] YANG, S., AND NACHLAS, J. A. Bivariate reliability and availability modeling. Reliability, IEEE Transactions on 50, 1 (2001), 26-35.

[54] YUN, W. Y., AND KANG, K. M. Imperfect repair policies under twodimensional warranty. Proceedings of the Institution of Mechanical Engineers 221, O4 (Dec. 2007), 239.

[55] YUN, W. Y., MURTHY, D., AND JACK, N. Warranty servicing with imperfect repair. International Journal of Production Economics 111, 1 (2008), 159-169. 